\title{
The International Bureau of weights and Measures 1875-1975
}

National Bureau of Standards 



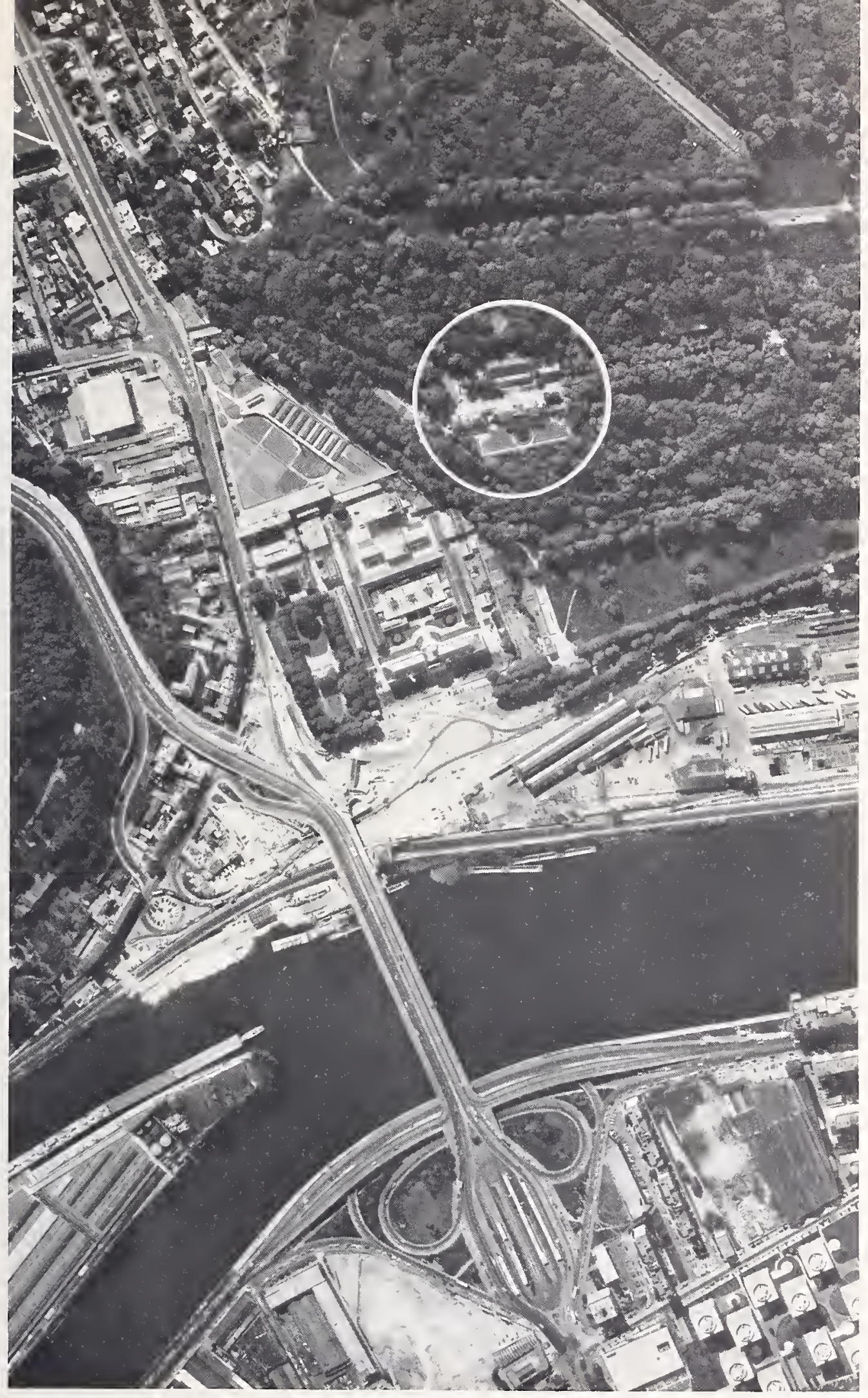

Acrial view of the Pavillon de Breteuil and the immediate environs. To the east, the Seine and the Pont de Sevres; to the northwest, the Parr de Saint-Clourl: between the Pavillon de Breteuil (eireled) and the lridge: the Manufacture Nationale de Porcelaine de Sèvres. The new lahoratorics (1964) are situated north of the circle and are searcely visible; they were built in a way to preserve the countryside. (Document Institute Comgraphioue National. Paris). 

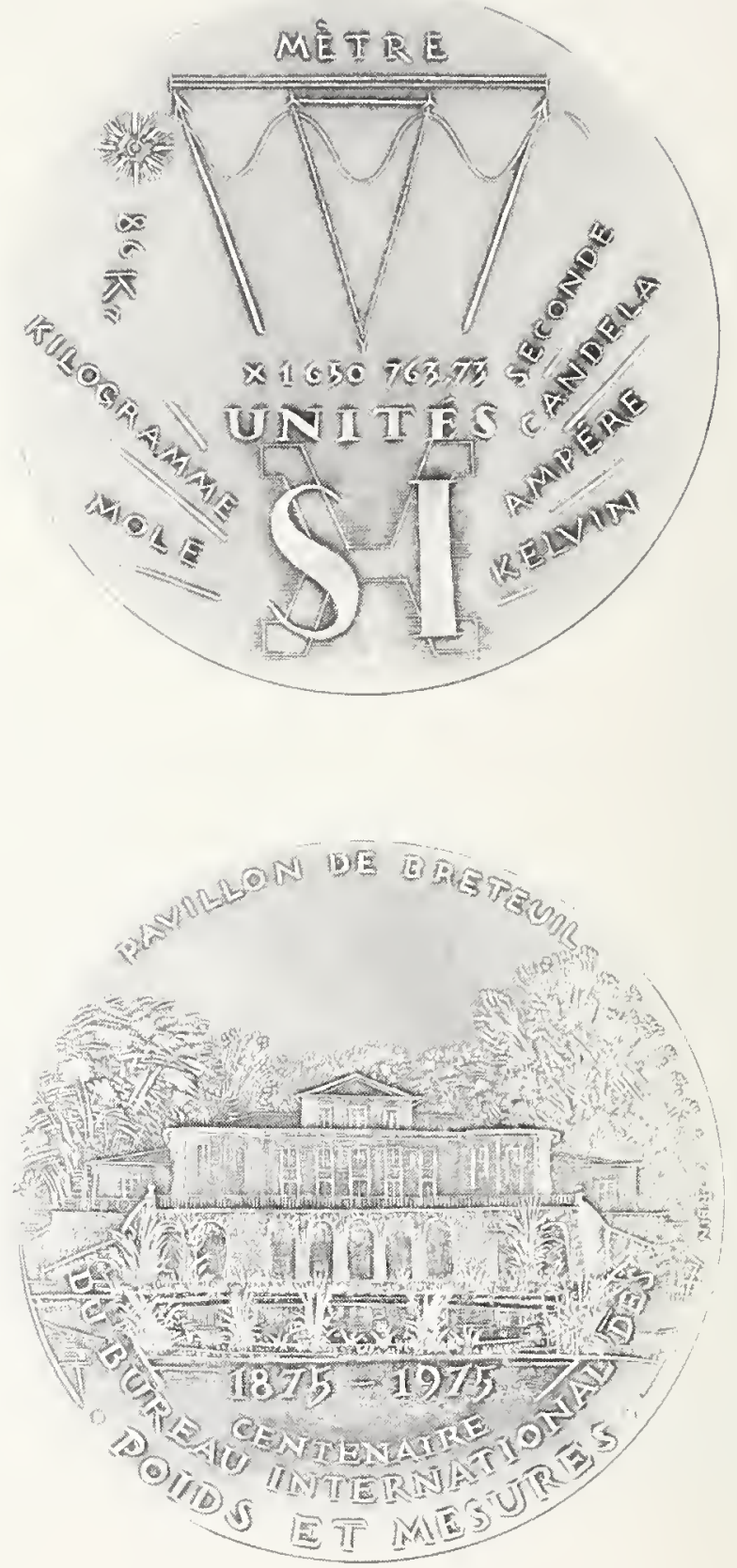

Medal commemorating the centemial of the Convention of the Metie and the International Bureau of Weights and Measures. (Designed by R. Corbin. Monnaie de Pais) 


\section{The International Bureau of Weights and Measures 1875-1975}

Edited by

Chester H. Page

National Bureau of Standards, U.S.A.

and

Paul Vigoureux

National Physical Laboratory, U.K.

Translation of the

BIPll Centennial Volume

Publi-hed on the oceasion of the

looth Anniversary of the

Treaty of the Metre

May 20, 1975

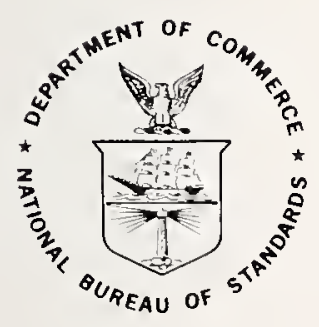

\section{U.S. DEPARTMENT OF COMMERCE}

NATIONAL BUREAU OF STANDARDS, Richard W. Roberts, Director

Issued May 1975 
National Bureau of Standards Special Publication 420

Nat. Bur. Stand. (U.S.), Spec. Publ. 420, 256 pages (May 1975)

CODEN: XNBSAV

U.S. GOVERNIIENT PRINTING OFFICE

WASHINGTON: 1975

For sale by the Superintendent of Documents

U.S. Government Printing Office, Washington, D.C. 20402

Paper cover Price $\$ 3.00$

Stock Number 003-003-01408

Catalog Number C13.10:420 


\section{FOREWORD}

The metric system was made legal by Congress in 1866, the United States of America signed the Treaty of the Metre in 1875, and we have been active in international coordination of measurements since that time. In science, in pharmacology, and increasingly in industry, the United States is making ever greater use of the International System of Units (SI).

In view of this ongoing conversion to the metric system, the celebration of both the Centennial of the Treaty and the first century of metrology at the International Bureau of Weights and Measures (BIPM) holds considerable interest for the American public. It therefore gives me great pleasure to make available this translation of the BIPM's official centennial volume.

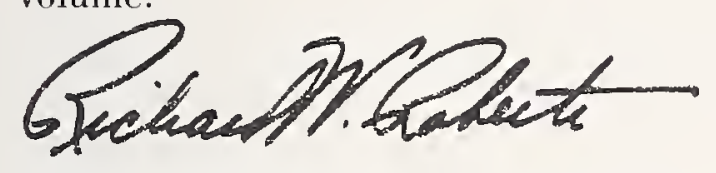

Richard W. Roberts

Director, National Bureau of Standards 



\section{THE INTERNATIONAL BUREAU OF WEIGHTS AND MEASURES AT THE TIME OF ITS CENTENNIAL}

We have all heard of the International Bureau of Weights and Measures; it is even one of the memories which have been part of us since our school days. And it is still more true in countries other than France, without doubt because there is added to the prestige of the "Standard Metre" and the "Standard Kilogram," that of the metric system conceived by the Paris Academy of Sciences under the French Revolution.

The International Bureau of Weights and Measures is situated on French ground, but it is independent of France: decisions concerning it rest with a Conference which assembles the delegates of 43 countries, furthermore, the 18 members of the Committee elected by the Conference, who constitute, with the Director of the Bureau, its operations directorate, must be scientific persons of entirely different nationalities. These 43 countries contribute, each its share, to the operating expenses of the BIPM, which was therefore since the beginning, and still is, truly international.

That which characterizes the BIPM now, at the time of its centennial, is the blending of permanence, which comes from its mission, and of rapid renovation, as in the techniques used to accomplish this mission. Its permanent mission is to achieve uniformity of physical measurements throughout the world, at the highest level of precision; this uniformity, recognized as necessary as early as 1875 , has become even more so since the advent of the rapid scientific and industrial progress which dominates our civilization. As for the level of precision, it raises itself today, by virtue of the discoveries in atomic physics, to a degree which would have been inconceivable 10 or 20 years earlier; the former standards of a mechanical nature tend to be replaced by standards of an atomic nature controlled by rigorous universal quantum laws. This is why the BIPM needs a staff of an ever higher scientific level; additionally, its technical equipment must be regularly updated. Thus, in the fields where it specializes narrowly, the BIPM keeps itself at the level of the best metrological laboratories in the world.

The BIPM furnishes standards to countries needing them, compares the standards of countries which are sufficiently advanced to establish their own standards, and contributes to research looking forward to the still higher precision of the future; it serves as a neutral and impartial scientific arbiter when national sensitivities impede an international agreement. It is 
also a center for discussion. for coordination. and for preparation of international decisions, whenever these decisions concern standards, for example, those of length and time (change of the definition of the metre in 1960 and of the second in 1967), or the International System of Units, developed from the Metric System. The importance of the mission of the BIPM is well illustratcd by the progrcssive adoption, in all countries, of the International System of Units; despite the force of habit which retards change, this system is spreading inevitably on its own merits, and is supplanting the multiplicity of disparate units which we have too long endured.

In the present volumc, the chapter on the measurement units of the International System of Units is due to Professor Jan de Boer (University of Amsterdam), Secretary of the International Committee on Weights and Measures. The history is by H. Moreau, metrological editor of the BIPM. The following chapters discuss the principal laboratory activities of the BIPM; they have been composed by its scientific staff. An editing committee, consisting of J. Bonhoure, P. Carré, G. Leclerc, A. Rytz, and P. Giacomo, deputy director, has seen to making them homogeneous in presentation and so easily readable that a large number of readers can absorb the most important aspects of the basic metrology, often ignored, sometimes even by scientists.

\section{J. Terrien}

Director, BIPM 


\section{CONTENTS}

Foreword

The International Bureau of Weights and Measures at the time of its centennial, by J. Terrien

From the earlier systems of measures to the International System of Units, by J. de Boer

I. HISTORICAL INTRODUCTION

The Metre Convention and its organs.

II. MASS.. .29

The Prototype Kilograms and periodic verifications

Determination of the mass of Kilograms outside the periodic verifications. $\quad 48$

Balances and methods used for the comparison of Kilograms; auxiliary

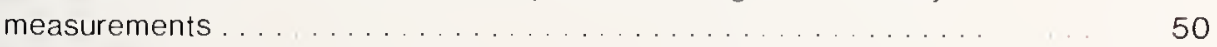

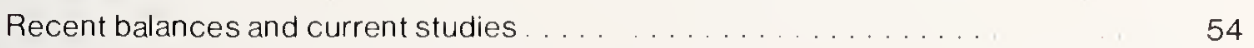

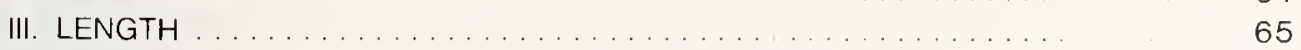

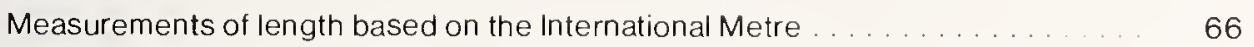

Toward the change of the definition of the metre . . . . . . . . . . . . . . . 72

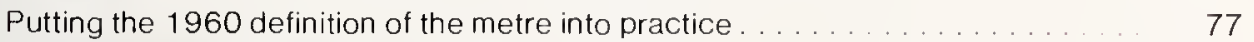

Complementary studies of the standard radiation . . . . . . . . . . . . 88

Lasers . . . . . . . . . . . . . . . . . . . . . . . . . . . . . . . . . . . . . . 92

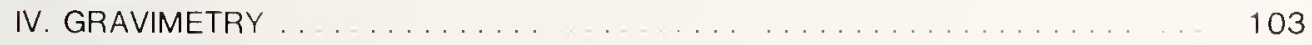

Metrological importance of the knowledge of the acceleration of gravity .... . 103

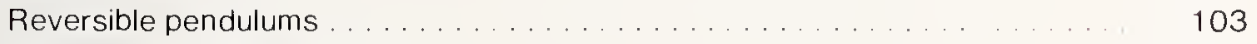

Free fall of a graduated scale . . . . . . . . . . . . . . . . . . . . 104

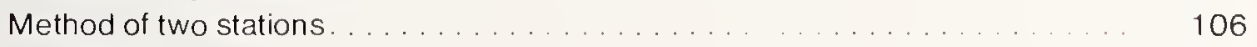

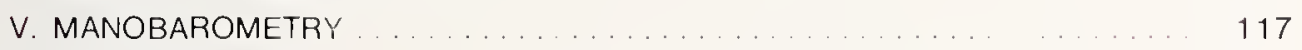

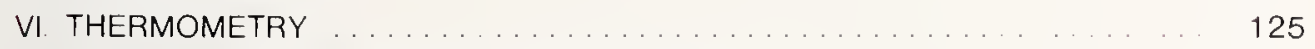

The gas thermometer and the mercury thermometer . . . . . . . . . . . . 125

The International Practical Temperature Scale . . . . . . . . . . . . . . . 126

Pyrometric study of the freezing-point temperature of gold . . . . . . . . . 131

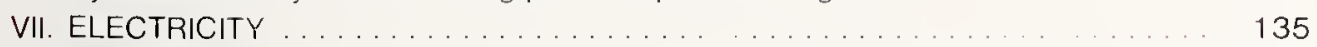

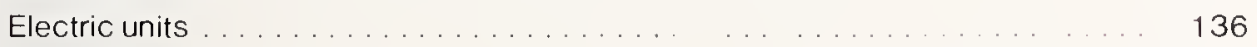

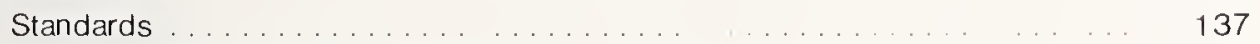

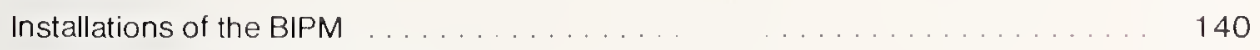

Periodic international comparisons. . . . . . . . . . . . . . . . . 145

Maintenance of the ohm and the volt by the BIPM $\ldots \ldots \ldots \ldots \ldots \ldots \ldots \ldots$

Progress of metrology in the field of electricity . . . . . . . . . . . . . . 154

VIII. PHOTOMETRY . . . . . . . . . . . . . . . . . . . . . . . . . . 163

Apparatus and measurement methods at the BIPM . . . . . . . . . . . . 164

International comparisons of intensity and of luminous flux . . . . . . . . . 170

Maintenance of the units and stability of the standard lamps . . . . . . . . . 173

Spectrophotometric measurements . . . . . . . . . . . . . . . . . . 173

International comparison of distribution temperature scales . . . . . . . . . 175

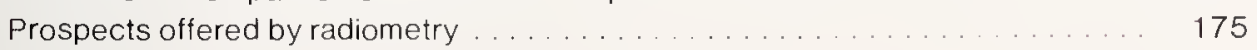


IX. MEASUREMENT OF RADIOACTIVITY . . . . . 179

Generalities on the methods of measurement . . . . . . . 179

The method of counting by $4 \pi \beta$ (PC)- $\gamma$ coincidences $\quad 180$

The BIPM laboratory for radionuclide measurements . . 181

Radioactivity standards ............................ 183

International comparisons of radionuclides. . . . . . . . . . . . . . . . . . 184

Statistics of counting . . . . . . . . . . . . . . . . . . . . . 185

Energy measurement: alpha-particle spectrometry . . . . . . . . . . . . . . . . . . . . . . . . . .

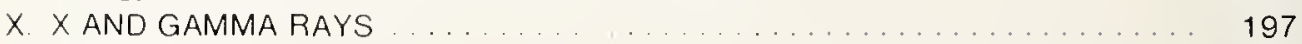

Generalities ... . . . . . . . . . . . . . . . . . . . . . . . . . . . . 197

Measurement of X Radiation. ......................... 200

Measurement of Gamma Radiation. . . . . . . . . . . . . . . . . . . 203

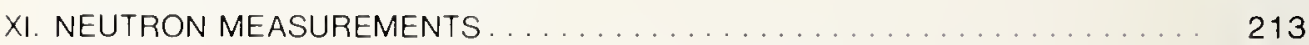

Generalities .................................. 213

Measurement of the emission rate of a neutron source

by the manganous sulfate bath method. . . . . . . . . . . . . . . . 215

Comparative measurements of neutron sources . . . . . . . . . . . . . 218

Measurement of the flux density of a source of fast monoenergetic neutrons . . . 221 Appendixes

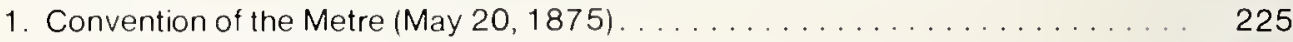

2. Member States of the Metre Convention . . . . . . . . . . . . . . . . . . 231

3. Members and officers of the CIPM, and Directors of the BIPM from 1875 to $1975 \quad 233$

4. The base units of the $S \mid$ and their origins . . . . . . . . . . . . . . . 238

5. Worldwide expansion of the Metric System . . . . . . . . . . . . . . . . 243

6. Publications of the Metre Convention Organs . . . . . . . . . . . . . . . . . 245

7. Abbreviations used in this volume . . . . . . . . . . . . . . . . . . . . . . 247 


\title{
FROM THE EARLIER SYSTEMS OF MEASURES TO THE INTERNATIONAL SYSTEM OF UNITS
}

\author{
J. de Boer
}

\section{Numbers and Measures}

On the occasion of the celebration of the centenary of the Convention of the Metre* it seems appropriate to review the origins and historical developments of unit systems and in particular of the International System of Units, one of the most important results of the broad international collaboration that has been realized in the framework of the Convention of the Metre.

The development of such an internationally accepted unit system is closely related with the history of science itself. The purely metrological aspects of the development of weights and measures and of measuring standards in general are of course directly influenced by science and technology. But also the logical structure of the system. the symbolical and mathematical formalism and the manner in which numerical statements are expressed are intimately connected with the status of the mathematical sciences at any given moment. Moreover. adequate methods to measure and to express results of measurements in a symbolic form arc also essential for the development of the expcrimental sciences such as physics, chemistry, and technology. There exists an intimate interaction which Cassirer expressed as follows: "Physical theories gain their definiteness from the mathematical form in whicl they are expressed. The function of numbering and measuring is indispensable even in order to produce the raw material of facts that are to be reproduced and unified in a theory."

So even a brief review of the history of such a unit system as the International System of Units - related with and deeply rooted as it is in the development of modern science and technology - necessarily has to pay at tention also to the symbolism for expressing numbers and numerical statements and to the whole set of formal symbolic rules which all together constitute the elements of a sophisticated system of units and which give it its central place in modern exact science.

\footnotetext{
* Editor's note: In the U.S.A. commonly called "Treaty of the Metre."
} 


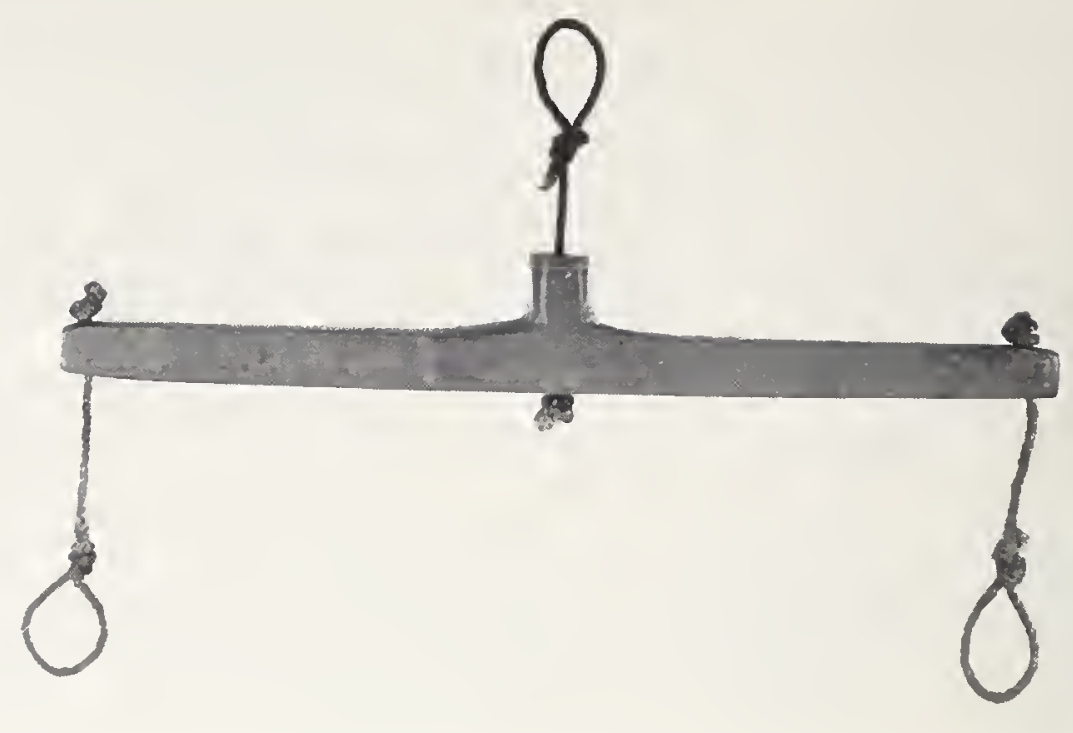

Balance of around 5000 B.C.. foumd in a prehistoric lomb in Naqrada in Egypt: length of beam $\approx 8.5 \mathrm{~cm}$ (Courtesy Petrie IIuseum. Lniversity College London).

In most of the early civilizations the economic needs for making quantitative measurements, for instance of areas of land, amounts of food, water, and materials, and for establishing reliable time scales and calendars, led in an early phase to the introduction of systems of counting and expressing numbers, and of various measures and measuring systems for a great variety of purposes. Obviously we are unable to go here into a detailed account of this early history; we have to limit ourselves to a few main points which are relevant for our subject.

Already in the early Egyptian system of measurcs. mainly of length, area, and volume, a decimal system of counting was used, with distinct symbols for the numbers 1, 10, 100, and 1000. The decimal system was also, though not systematically, used for expressing multiples of their basic measures: their basic length unit was the "meh" or cubit; the "khet" is equal to 100 cubits. The "sethat," the unit of area of land, is equal to a square khet, etc. However, for the submultiples of the cubit no decimal fractions were used: thus for instance the "shesap" (or handpalm) and the "djeba" (or finger) were equal to $1 / 6$ and $1 / 24$ of a cubit respectively.

It is well known that the Sumerians and Babylonians used a sexagesimal system, which had in fact many advantages because of the easy divisibility of the number 60 by $2,3,4,5,6,10,12$, etc. The very high level of astronomy and advanced status of time-measurement in these early Mesopotamic cultures and the influence which this early development had in the later development of science have resulted in the absorption of essential features of this sexagesimal system into western civilization. We still divide 1 hour into 60 minutes and 1 minute into 60 seconds and these 
units are so universally used that they had to be maintained as such for use with the present International System of Units!

However, their system of measures did not make systematic use of sexagesimal division: thus for instance one "kus" (cubit) was divided into 30 "shusi" whereas 12 kus made a "gar" (comparable to the pole), which was frequently used for land measurements.

A very important new development appears in the cuneiform texts of the Sumerians: their symbol for 60 was the same as that for 1 and the value to be attributed to this symbol depends on its position in the number. So the Sumerians really introduced positional notation in writing nmmbers, contrary to the Egyptians who repeated the symbols for 1, 10, 100, etc., as many times as was needed to symbolize large numbers. This invention of positional notation is well known to have been of the utmost importance for the simplification of numerical calculations, but it would still take a long time before the decimal system of the Egyptians was combined with the positional notation.

What was needed here were separate symbols for the numbers 1 to 9 and for zero, which were introduced in India in the first centuries, A.D. From these Indian symbols originate the Western Arabic figures, from which our figures have been derived, as well as the Eastern Arabic figures, which have developed into the present Arabic notation. The oldest inscriptions using a real decimal and positional notation with Indian figures date from around A.D. 600. The zero seems to have come into use only a few hundred years later.

The introduction of these figures in Western Europe still gave rise to considerable difficulties. In fact, Europe inherited from Rome the "Roman" figures with their complex juxtapositional notation and the use of the abacus for numerical calculations. The Indian-Arabic figures with positional notation for the numbers 1 to 9 and the zero sign, became known through the books on Indian calculation from the Arab writer Al-Khwarizmi, which were translated into Latin in the 12th century by an Enghish monk. The reaction to the use of Indian-Arabic figures in Western Europe was not always favorable: in fact their use instead of the usual Roman figures was often forbidden by the banks, which feared confusion. The struggle between the "abacists" and the "algorithmicists," supporters of the abacus and of Al-Khwarizmi respectively, continued up to the 15 th century: but gradually people in Europe became convinced of the superiority of the system of Arabic figures with positional notation, which we use at present.

Before leaving this subject, it should be mentioned that a separate ef fort was yet needed to introduce the decimal notation for fractions. Figures like $8 \mathrm{l} / 4$ and $1373 / 8$ were usually used instead of 8.25 and 137.375. Many claims for the invention of the decimal writing of fractions have been made - the Chinese used decimal fractions already around A.D. 1200-but it is generally agreed that very much of the credit for its introduction in Eu- 
rope has to go to Simon Stevin from Lciden who published in 1585 his famous essay "De Thiende" (The Tenth), very soon translated into English and French. In this essay decimal fractions in positional writing were introduced and the mokern calculus with decimal fractions was devcloped in great detail. John Napier, the inventor of the logarithms, was responsible for introducing the decimal sign. Another very important contribution of Simon Stevin was his request to replace the cxisting great variety of fractions and multiples of existing measures by decimal fractions in the measures for lengths, surfaces, and capacities as well as in astronomy (angles!) and in money systems. This proposal was certainly a very clear example of the foresight which he had for the needs of a good measuring system in the future. Indeed, the main prerequisites in the field of calculation, which were needed for setting up a good system of units, had now been obtained.

After having gone, very briefly, into the history of numbers - an essential element of the precise expression of the results of measurements - we now return to the measures themselves.

In fact, most of the early practice in Europe in making quantitative statements in the field of weights and measures was inherited from the Romans. Their weights and measures were based on the basic units of length and weight, the "pes" or foot and "libra" or pound respectively. The unit of capacity, the "amphora," was equal to one cubic pes. But for land measurements the square pes was too small; in fact the Romans used the "jugerum" which was equal to twice the area of a square on a side of 120 pedes. In addition a great many other units were introduced which were simple fractions and multiples of these units, so that no systematic use of decimal multiples and submultiples appears in these Roman weights and measures, contrary to their decimal system of counting. Many of these Roman weights and measures were taken over in the civilization of Western Europe, but the rather feudal structure of the society, developing after the breakdown of the Western Roman Empire, led to a large diversity of local customs, of weights and męasures and rules, which was a source of much confusion. Already in 789 Charlemagne had promulgated a decree on the unification of measures in all countries under his crown but unfortunately this attempt disappeared with the empire of Charlemagne. The development of a new society, following the growth of important and independent towns in Italy, France, Germany, and other countries starting in the 14th century, and the corresponding growth of an economy, based on manufacture and money, and on trade relations over land and over sea, was a stimulus for the growth of science: mathematics, astronomy, mechanics, and the technical sciences. The quantitative aspects of life became more and more important and as a consequence weights and measures were also looked at more carefully. Several attempts were made in the great kingdoms of the 15th and 16th centuries to introduce well defined systems of weights and measures. 

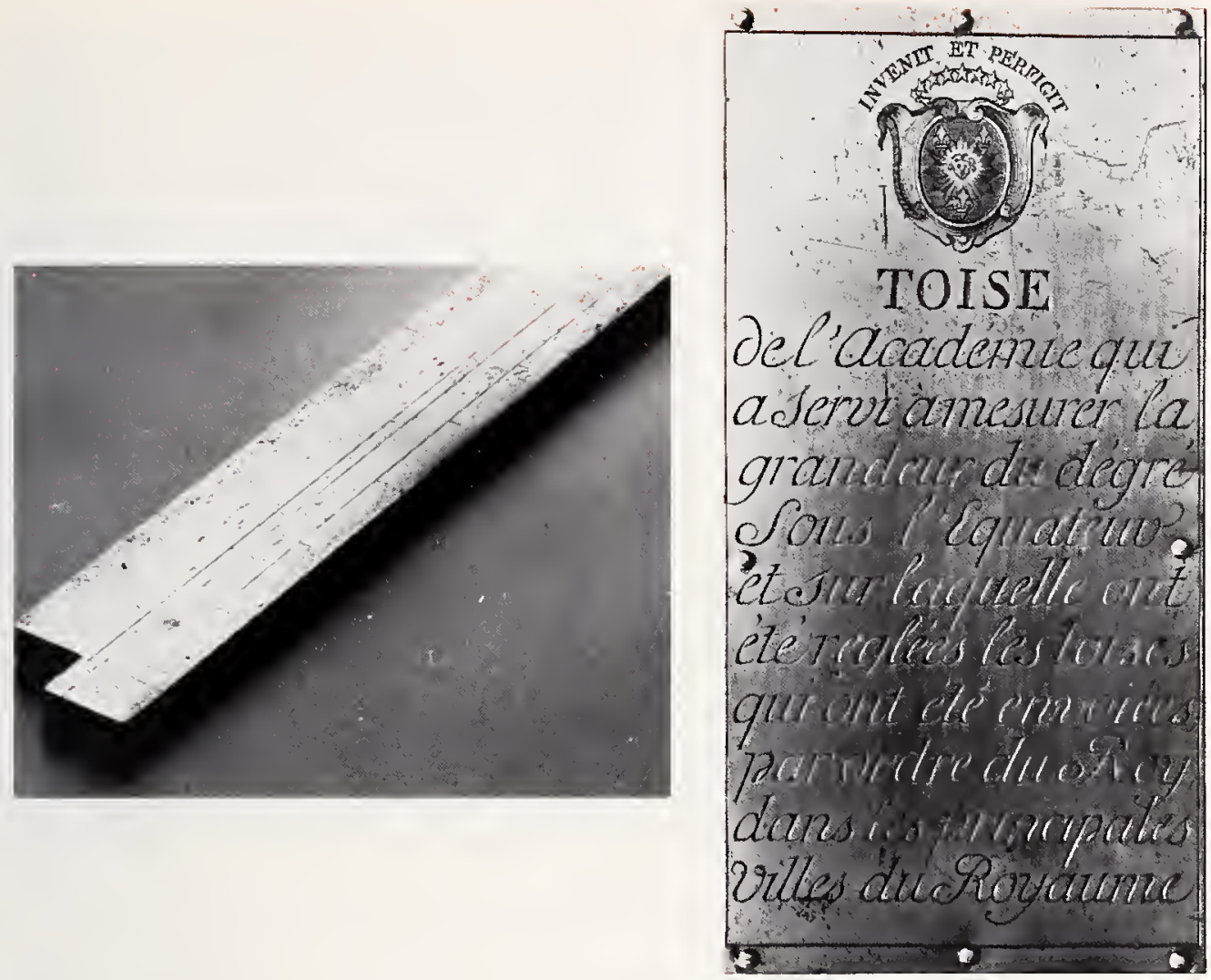

"Toise du Pérou" from the "Académie," conserved in the "Observatoire de Paris," On the right. the plate attached to the case.

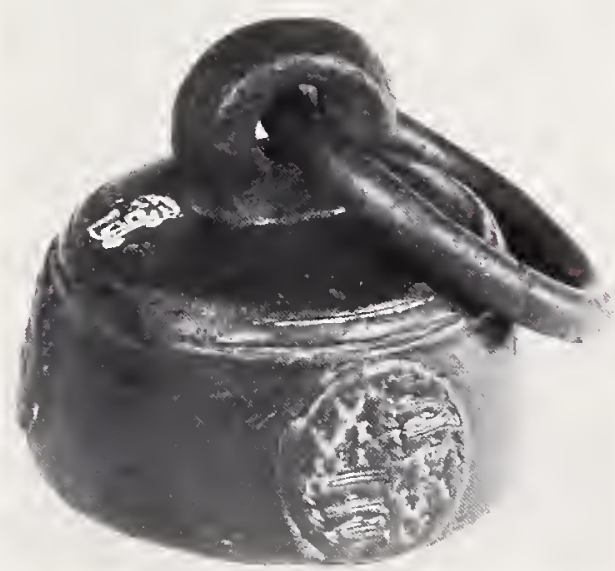

"Averdepois" weight of Edward III. (British Crown Copyright. Science Museum, London.) 


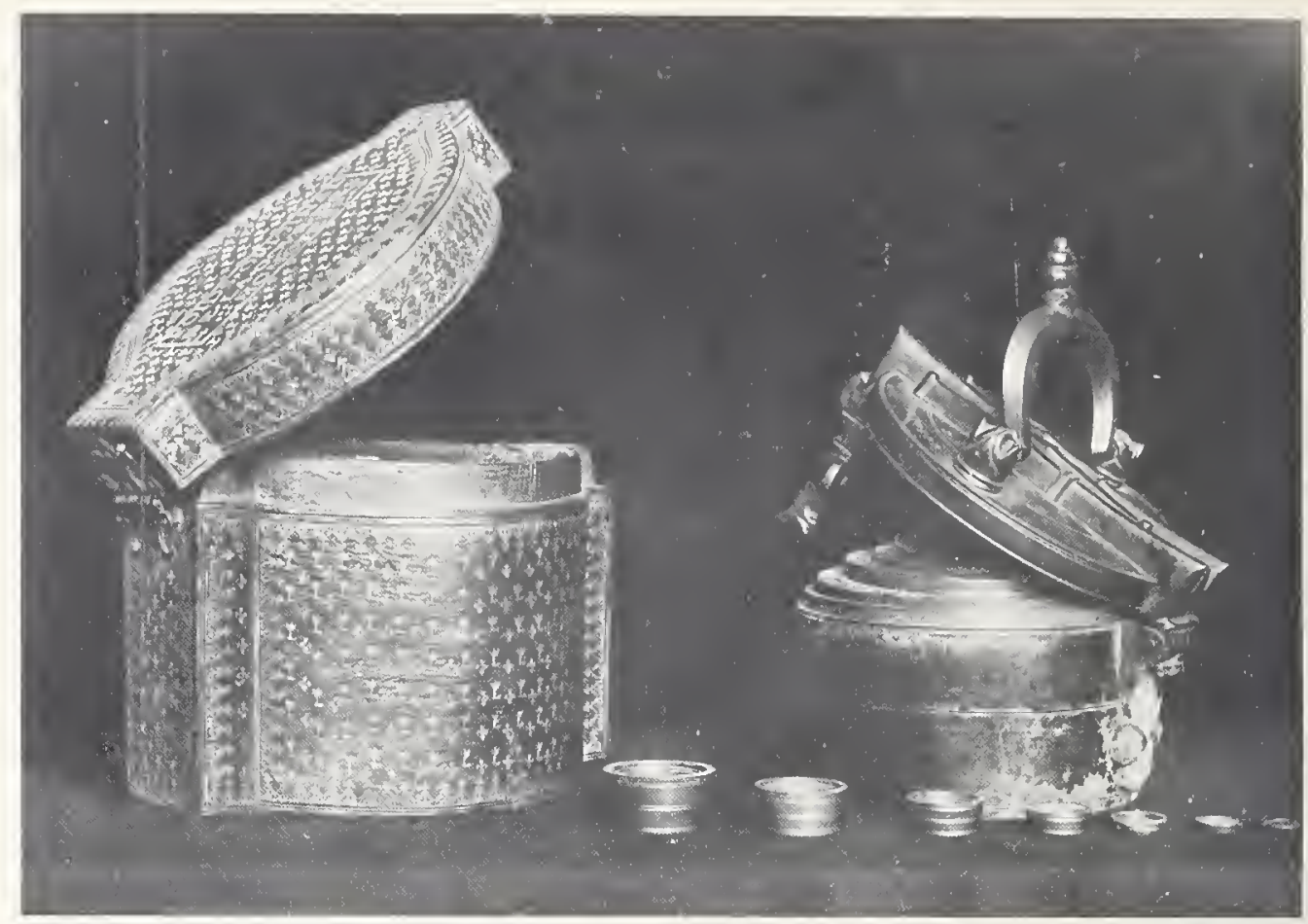

"Pile de Chatemagne" with its case beaning the inscription "lenids original de la Cour des Monoyes" (Muséc National des Techniques. Paris).

In France, at the end of the 15th century, the "Pile de Charlemagne" defined the "Livre poids du marc" $(490 \mathrm{~g})$ and its (nondecimal) subdivisions; and around 1670 the "Toise du Chatelet" was introduced, one sixth of the length of which defined the length of the "Pied du Roi" (32.5 $\mathrm{cm})$, the French foot, in terms of which the "pouce," the "ligne" and the "point" were defined by successively dividing by twelve. In England Queen Elizabeth I introduced at the end of the 16th century the "avoir du poids" weight of 1 pound (453 g) divided into 16 ounces and the yard divided into 3 feet $(30.5 \mathrm{~cm})$, each foot being 12 inches. But in spite of these valuable at tempts to unify weights and measures the confusion persisted: in fact even the French government never succeeded in imposing the "Parisian" units on the whole country, and so in the whole of Europe an infinite variety of weights and measures continued to exist.

\section{Origin of the Metric System}

So it is not surprising that in the course of the 17th and 18th centuries not only leading people in government circles, but also scientists who were making the first important steps in developing the modern picture of experimental sciences, became more and more disturbed by the existing disorder and uncertainty as regards the various weights and measurements, which they too had to use to express their experimental results. In 1742 a 
group of scientists made a careful comparison between the so-called "Paris measures" and those used in England at that time, with the result that the French "pied" and "livre" were found to be larger than the English "foot" and "pound" by 6 and 8 percent respectively. Scientists started to search for a suitable universal unit - not bound to any nationality - on which a system of measurements, identical in all countries, could be based. Two very different propositions were made for the choice of the basic unit of length: one being the length of a seconds pendulum and the other being a basic length related to the length of an arc of the meridian. The first proposal had already been made by the French astronomer Mouton (1670) and by Huijghens (1673) and was supported by the Royal Society of England. In France Talleyrand in 1790 made a formal proposal to the Constituent Assembly in this same direction, but the French Academy of Sciences rejected the proposal for the well known reason that the length of the seconds pendulum depends on the gravitational acceleration and therefore differs from place to place on the earth. The Academy favored the alternative proposal and on its recommendation the General Assembly adopted in 1791 the principle of a system of weights and measures founded entirely on one base unit of length, the "metre," defined to be equal to one ten-millionth of the length of the quadrant of the earth meridian. Units of area and capacity would be decimal multiples and submultiples of the square metre and the cubic metre respectively and the unit of weight (or mass) would be the weight (or mass) of a cubic decimetre of water at the temperature of maximum density $\left(4^{\circ} \mathrm{C}\right) .^{1}$ The system would have to be entirely decimal, using prefixes like milli (1/1000), centi (1/100), deci $(1 / 10)$ and deca (10), hecto (100) and kilo (1000) to be added to the names of the units to indicate submultiples and multiples. Because of its foundation entirely on the metre this system got the name "Système Métrique," the name through which the system would gradually spread all over the world. Unfortunately, however, the English and American governments considered the French proposal impracticable and decided to derive their basic unit of length on the seconds pendulum, so that the French people had to pursue their goal alone.

Soon after this decision Delambre and Méchain measured the arc of the meridian between Dunkerque and Barcelona whereas Lavoisier and others made careful measurements of the weight (or mass) of a known volume of water. On the basis of these measurements an end standard made of sintered platinum representing the metre and a platinum standard kilogram were constructed and then deposited as such on June 22, 1799 in the Archives de la République in Paris. These two standards really would be the starting point for the whole development of the present universally adopted International System of Units. Already in 1793 Lavoisier said:

1 The Romans had alrealy chosen their unit of capacity, the amphora, such that the amount of water filling the amphora weighed exactly an attic talent. 


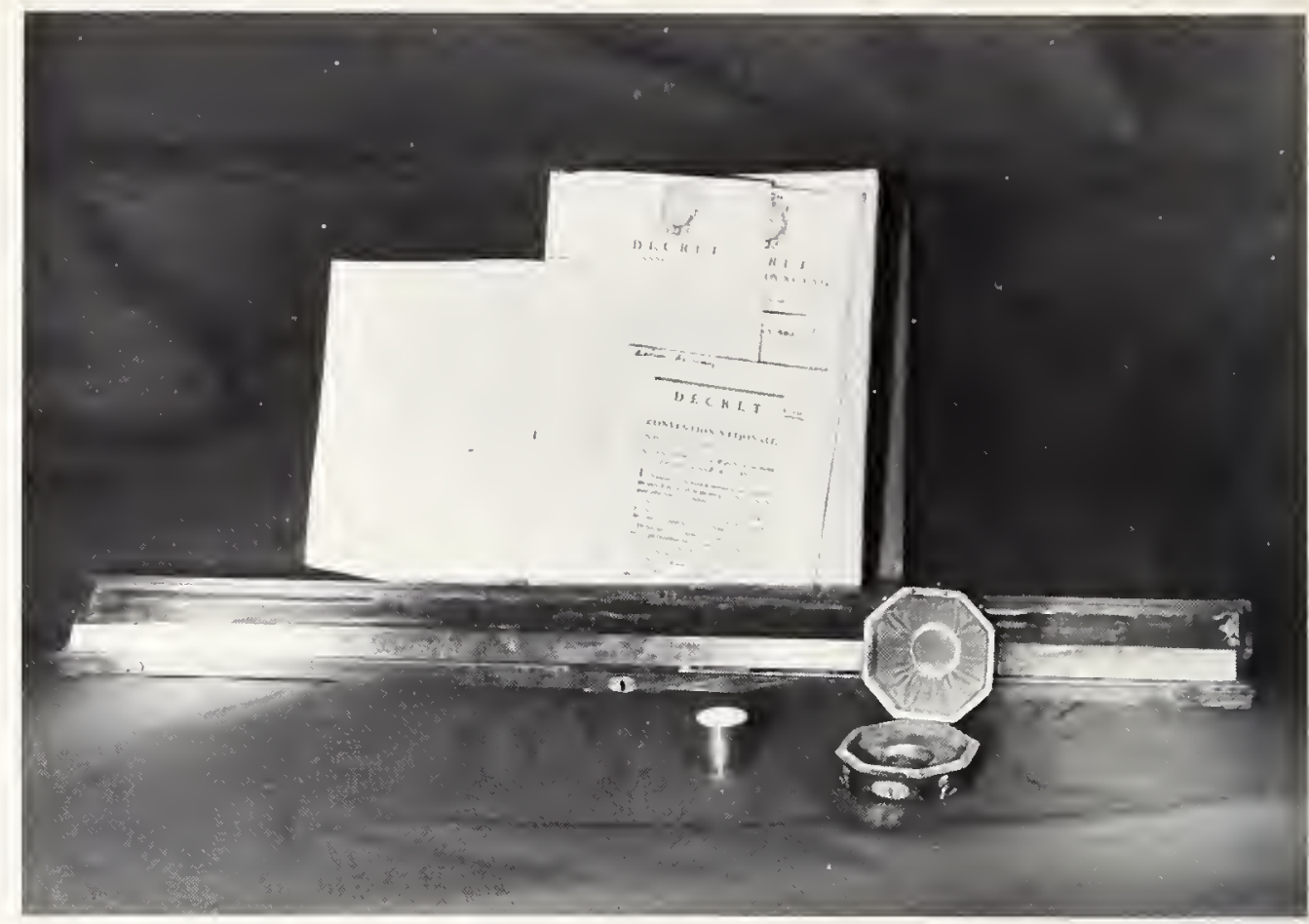

Metre and Kilogram "of the Archives" (1799) with their cases bearing the inscription "Conforme à la Loi du 18 Germinal an 3. présenté le 4 Messidor an 7," and decrees of 1793 and 1795 instituting the Metric System in France (Photo Archives Nationales, Paris).

"Never has anything grander and simpler and more coherent in all its parts come from the hands of men."

However - from the formal point of view - it has to be remarked, that the final result: the metre and kilogram "des Archives," two basic standards for length and weight (or mass) which were legally given the values of 1 metre and 1 kilogram respectively, in fact represented a system of weights and measures based on two material standards, which in the future might easily be found to deviate from their origin: the length of the quadrant of the earth meridian and the simple numerical value for density of water agreed upon by definition. In fact it was found later than the "Mètre des Archives" is about $0.2 \mathrm{~mm}$ shorter than one ten-millionth of the length of the quadrant of the meridian and that the mass of the "Kilogramme des Archives" is equal to that of 1.000028 cubic decimetres of water - at the maximum density - instead of 1 cubic decimetre exactly. ${ }^{2}$

2 This small difference has been the source of long controversy about the ratio of the litre, defined as the volume of $1 \mathrm{~kg}$ of water at its maximum density, to the cubic decimetre; this ratio is given by the same number 1.000 028. In 1964, the litre was finally defined to be exactly $1 \mathrm{dm}^{3}$. 


\section{The Metre Convention}

With the initiative of the French people the starting sign had been given for a triumphal tour of the metric system all over the world. Already in 1820 the system was introduced by law in the Low Countries (Holland, Belgium, and Luxembourg). Spain, Colombia, Mexico, Portugal, Italy, and many other countries followed. In 1864 the use of metric weights and measures concurrently with those of the imperial system was authorized in Great Britain and a few years later the metric system was introduced in Germany.

During the world exhibition of Paris in 1867 a great many scientists, impressed by the tremendous industrial development in tle world, created a Committee for Weights and Measures and for Money, with the special task of creating more uniformity in the world. Following this valuable initiative the French government in 1869 invited numerous countries to send delegates to Paris for an "International Commission for the Metre." A total of 24 countries appointed delegates and the meetings started in August 1872. The great majority of those present at the meeting felt that the metre and kilogram which were conserved in the "Archives de Paris" should be the reference for new prototypes and for various copies to be constructed and distributed among the participating countries.

Unfortunately the work was interrupted by the Franco-Prussian war but in 1872 the same Commission, then consisting of delegates represcnt ing 30 countries, met again and confirmed its previous decision to construct new prototypes of the metre and the kilogram and to provide a large number of copies, all made from one single melt of a special platinum-iridium alloy with 10 percent iridium. The standards of the metre should be line-standards with an X-shaped cross section. The values of the new prototypes should be based on the metre and the kilogram in the "Arclives."

On the 1st of March 1875 the French Government convened the "Conférence Diplomatique du Mètre" to which 20 countries had sent government representatives and scientists who were authorized to sign for their governments. These delegates approved the proposals made by the Committee, so that finally on the 20th of May 1875 the "Convention du Mètre" could be signed officially.

The newly established "Comité International des Poids et Mesures" concentrated on the preparations for the construction of the prototypes and the various copies of the metre and the kilogram. Many very serious difficulties had to be overcome and it was a real scientific accomplishment of the first order when in 1899 at the first "Conférence Générale des Poids et Mesures" the national prototypes could be distributed and approval given to the two prototypes for the metre and the kilogram which from then on were considered to form the basis for the metric system. These prototypes were to be conserved in the "Bureau International des Poids et Mesures," which should be the central laboratory for future international comparisons 
and which should be the international center for the propagation of the metric systcm. The International Bureau should work under the direction and exclusive supervision of the International Committec which itself is subordinated to the Concral Conference.

We will not follow the history of the International Bureau of Weights and Measures and its further cxpansion togcther with the governing bodies: the International Committee and the various Consultative Commit tees. This will be dealt with elsewhere in the present volume. Our aim is to review briefly the development of the scientific ideas about units and how they have to be related and combined into a unit system.

\section{The Concept of a Unit System}

The two or three cent uries preceding the signing of the metre convention had been a period of tremendous scientific development in which the role of physics as the most advanced experimental science had become more and more pronounced: physics had developed into a real science of measurements, in which the concept of "physical quantities" is introduced; these are mathematical entities, such as mass, force, energy, etc., connected with and describing the physical phenomena. They are defined in terms of actual measurements and their introduction leads to a description in terms of mathematical relations between these physical quantities, which can be looked upon as definitions or as physical laws, although this distinction is generally difficult to make.

According to Maxwell every physical quantity can be expressed as the product of a pure number and a unit, where the unit is a selected reference quantity in terms of which all quantities of the same kind can be expressed. The ratio of the quantity to the corresponding unit, which is a number, is usually referred to as the numerical value of the quantity expressed in that particular unit. The unit as introduced here thus has the same kind of abstract mathcmatical character as the corresponding physical quantity it self. Such abstract units should therefore not be confused with their explicit physical realizations, which are usually called measurement units or standards.

Physics had become the prototype of an exact science. Obviously the choice and the definition of the units for the various physical quantities and the way they are integrated into this mathematical description as a "unit system" are extremely important. The experimental and metrological aspects of physics and the various related engineering sciences require an ever increasing experimental accuracy, which leads to a continuous need to improve the precision of the measuring standards representing the various units. On the other hand the theoretical and mathematical aspects of the physical sciences require the units to constit ute a logical system, which itself is an integral part of the whole mathematical formalism through 
which the various relations between physical quantities and the laws of physics are expressed. We briefly sketch some important steps which seem to be essential in the long and often very curved road, which runs from the beginning of the French Metric System up to the present more sophisticated International System of Units.

In the second half of the last century, the British Association for the Advancement of Science (B.A.A.S.) played an important role in developing the concepts relating to unit systems. In particular the Standards Commit tee has been a center of very fruitful cooperation of a great many famous scientists of that time, among whom Maxwell. in particular, should be mentioned. This Standards Committee studied around 1860 the best choice for the units corresponding to the various physical quantities such as electric charge, current, resistance, capacitance, and potential, which had been introduced in the theory of electricity. The Committee made clear that when a unit corresponding to a particular physical quantity has to be defined, there are always two questions to be answered: one should first determine what would be the most convenient unit and then second, what would be the best form and material for the standard physically representing that unit.

Although in principle one would be able to choose a unit and a corresponding standard for every kind of quantity it would be very inefficient to choose all the units independently. We have seen that already at a very early stage people felt that, for instance, the unit of area should be chosen to be the square of the unit of length. Only because of reasons of convenience, multiples or elementary fractions of this more systematic choice often came into use. In the metric system the logical choice for the unit of velocity is the metre per second, and the kilometre per hour which is so widely used at present is not "coherent" with this system.

\section{The CGS-Unit System}

The B.A.A.S. Standards Committee stressed the need for a coherent unit system which is built up systematically on a certain number of "fundamental" units - or base units as they are called now. The three base units for mechanics were chosen to be the centimetre, the gram. and the second of mean solar time, the centimetre being chosen instead of the metre because in fact one cubic centimetre-and not one cubic metre-of water had a mass of approximately one unit of mass (or gram) at the temperature of maximum density of water. The corresponding base standards of length and mass were accepted to be those conserved in Paris, whereas the definition of the time scale was left to the astronomers. This was the birth of the CGS-system, the first system of units conceived as such and still very much in use in physics. 
The development of physics into an exact science governed by a mathematical system of equations, thus also has its implications for the units. Only a limited set can be arbitrarily chosen and once the choice of thesc base units has been made, the whole system of units can be built up systematically.

One number of base units on which the physics of mechanical phenomena could be based appeared to be three, and the actual choice of the three fundamental quantities length, time, and mass - on the units of which the CCS-system was based-agrecd with what intuitively had already been the selection for centuries. Often such a system is called a threc-dimensional system, the number three referring here to the number of independent base units or quantities on which the system was based.

Once the three base units are decided upon, the requirement of coherence is sufficient to define all the other units, at present called derived units, in contrast to the base units. Thus for instance the physical quantity "velocity" is defined as distance travelled per unit time and as a consequence the "coherent" unit is defined to be the centimetre per second (cm/s), the physical quantity "momentum" is defincd as mass times velocity so that the coherent unit is the gram centimetre per second ( $\mathrm{g}$. $\mathrm{cm} / \mathrm{s}$ ), "force" is defined as increase of momentum per unit time with the unit $(g \cdot \mathrm{cm} / \mathrm{s}) / \mathrm{s}$ or $g \cdot \mathrm{cm} / \mathrm{s}$ " and "work" is defined as force times length of path with the unit $\left(\mathrm{g} \cdot \mathrm{cm} / \mathrm{s}^{2}\right) \cdot \mathrm{cm}$ or $\mathrm{g} \cdot \mathrm{cm}^{2} / \mathrm{s}^{2}$. In this way for every quantity, following its definition in terms of other quantities, the corresponding unit can be coherently defined. Any coherent unit system built up in this way is based on the system of equations which relate the various physical quantities.

New problems arose when electrical phenomena had to be taken into account. Already in 1832 Gauss - in his famous publication "The intensity of the earth's magnetic force reduced to absolute measure" - stressed the need to replace geomagnetic measurements with a magnetic needle by "absolute" measurements in terms of mechanical units of force. For that purpose Causs introduced an "absolute" unit system for the electrical units based on the millimetre, the milligram, and the second. Gauss was supported by Weber who extended his work to other electrical measurements. In England William Thomson, later Lord Kelvin. made similar measurements in the early fifties - measurements needed for telecommunication-in terms of the British mechanical units. In 1863 Maxwell wrote: "The phenomena by which electricity is known to us, are of a mechanical kind and therefore they must be measured by mechanical units or standards." The B.A.A.S. Standards Committee recommended following the same scheme and in particular extending the CGS-unit system by defining appropriate units for all electric quantities by just following the equations, developed by Faraday, Thomson, Maxwell. Weber, and many others, expressing them in terms of mechanical quantities length, time, force, and work. The units so obtained were called "absolute" in the sense of 
"coherent." i.e., they were not arbitrarily chosen but were simply the consequence of the system of equations by which the electrical and magnetic phenomena are represented. Unfortunately the electrical and mannetic phenomena could actually be described by two-mutually incompatible-systems of equations, depending on whether one started from the inverse-square distance force law between two magnetic poles or from that between two electric charges. For each of these two systems of equations Weber defined in 1851 a coherent "absolute" unit system and he called the two "absolute" CGS-unit systems: the electromagnetic and the electrostatic systems. The electromagnetic CCS-unit of electric resistance thus turned out to be one centimetre per second and already in 1851 Weber had performed beautiful experiments to determine the electric resistance of a wire in this "absolute measure."

The Standards Committee adopted in 1863 a method proposed by W. Thomson for such an "absolute" resistance measurement, with which method successful experiments were performed by Maxwell, Stewart, and Jenkin. With these measurements of the electric resistance a standard resistance in the form of a resistance coil could be prepared, the value of which in electromagnetic CGS-units would be obtainable with a sufficient precision of about $1 \mathrm{in} \mathrm{10,000.} \mathrm{Once} \mathrm{this} \mathrm{electric} \mathrm{resistance} \mathrm{standard} \mathrm{would}$ be available the measurement of all other electric quantities such as electric current and electromotive force in terms of electromagnetic CCS-units was considered to offer no more serious difficulties.

\section{Practical Electric Units}

However, the size of the electromagnetic CGS-units proved to be much too small, which was the reason that the B.A.A.S. recommended the use of certain "practical" units, i.e., the ohm for resistance and the volt for electromotive force, which were equal to $10^{9}$ and $10^{8}$ times the corresponding electromagnetic CGS-units respectively. The First International Electrical Congress approved this decision in Paris in 1881 and even introduced one more practical unit: the ampere for electric current, which was equal to one tenth of the corresponding electromagnetic CGS-unit. Later on the coulomb for electric charge and the farad for capacitance and several others were added.

This set of practical units actually also formed a "system" in the sense that they constituted a system, coherent with the system of equations connecting the electrical quantities with each other, but not with those in which mechanical quantities like force or work occurred. For instance the practical units of work, the volt-ampere-second, called joule, was equal to $10^{7}$ CGS-units of work. The introduction of these units, needed for practical purposes, thus spoiled the beautiful coherence of the original electromag- 
netic CGS system: only a limited coherence of the electric units among themsclves was left.

There was, however, another very disturbing development: so far the definitions of the practical units were still "absolute" definitions in the sense that they were still given - with integral powers of ten - in terms of the (absolute) clectromagnetic CGS-system. However, the material standards of the ohm (a mercury column), the volt (the Weston cell) and the ampere (silver voltameter), which were uscd for practical purposes as secondary standards, started to live their own life: at the International Electrical Congress in 1881 and more clearly at that in Chicago in 1893, the ohm, the volt, and the ampere were "legally" defined in terms of these material standards. Although the numerical values of these material standards expressed in electromagnetic CGS-units did not deviate very much from the integral powers of ten, it was nevertheless clear that the principle of this decision to define the electric units in terms of independent material standards, was quite contrary to the coherence principle used so far by the B.A.A.S. in their "absolute" definitions. However, the International Electrical Congress in London confirmed these new developments in 1908 and even adopted for legislation purposes and for metrology a complete system of these so-called "international" electric units based on the material standards of the ohm and the ampere. It would still take more than 25 years before this decision could be reversed, but we first have to pay attention to various developments which took place in the description of electrical phenomena.

\section{The Giorgi Proposal}

In the beginning of this century very important new ideas came up about the theoretical presentation of the electrical and magnetic phenomena and the construction of its unit system, which ultimately would lead to a new choice for a coherent unit system covering the whole field of electrical, magnetic, and mechanical phenomena.

Already in 1901 Giorgi remarked that the electric unit of work, the joule or volt-ampere-second, which is equal to $10^{7} \mathrm{CGS}$-units of work or $10^{7}$ $\mathrm{g} \cdot \mathrm{cm}^{2} / \mathrm{s}^{2}$, is in fact equal to 1 MKS-unit of work or $1 \mathrm{~kg} \cdot \mathrm{m}^{2} / \mathrm{s}^{2}$. However, simply replacing everywhere the centimetre and the gram by the metre and the kilogram in the "absolute" definitions of the electric units does not give the practical units because, for instance, the ohm which was defined to be $10^{9}$ electromagnetic CCS-units or $10^{9} \mathrm{~cm} / \mathrm{s}$, is equal to $10^{7} \mathrm{~m} / \mathrm{s}$ and not to 1 $\mathrm{m} / \mathrm{s}$.

Giorgi showed that this difficulty could be solved by increasing the number of fundamental units or base units on which the unit system for mechanics, electricity, and magnetism should be based from three to four base units: the metre, the kilogram, and the second of the MKS system. 
together with one base unit of electrical nature, for instance the ampere or the ohm. But the requirement of coherence of the unit system with the system of equations relating the various physical quantities then makes it necessary also to add to the three base quantities length, time, and mass. a fourth base quantity of electrical nature. This means that the electric quantities should no longer be defined in terms of the three "mechanical" quantities length, time, and mass exclusively - typical of Maxwell's formulation of the electromagnetic theory - but that in the force law for the magnetic interaction, which Maxwell had considered to be the starting point for the definition of the magnetic and electric quantities and their electromagnetic units, a constant of proportionality, the vacuum permeability, now had to be introduced. The value of this constant followed from the independent choice of the electric units to be $10^{-7}$ MKS-units of force per ampere squared.

It would take some time for the ideas of Giorgi to find acceptance among physicists and in electrical technology. The original Maxwell formulation of the theory, based on the mechanical quantities alone, found-and even at present still has - its active supporters. Still there was much intuitive logic in the request to increase the number of base quantities by one in going from mechanics to the description of the electrical phenomena. One might say that basic for any theory in physics are the base quantities for length and time. Characteristic for the mechanical dynamics is the concept of mass, which connects force and acceleration and which determines also the gravitational interaction. In electricity a similar quantity - of the same kind of fundamental nature - is the quantity electric charge or current. As long as this quantity is not shown to be of mechanical nature it plays in electricity a role similar to that of mass in mechanics, so that the total number of base quantities should increase to four. Consequently also the number of units on which the coherent unit system should be based, should be four.

Another indication of the insufficiency of the Maxwell presentation of all electric quantities and units in terms of mechanical ones alone was the fact that the formal unit expressions often carried fractional exponents, e.g. . the electromagnetic CGS-unit of electric current: $\mathrm{cm}^{1 / 2} \cdot \mathrm{g}^{1 / 2} / \mathrm{s}$, or the occurrence of rather strange unit expressions like the $\mathrm{cm} / \mathrm{s}$ for electric resistance. These are all clear indications that the chosen number of base quantities and units is too small. The only conclusion could be that in the future the equations of Maxwell ought to be rewritten in four-dimensional form, i.e., with four base quantities-including one of electrical nature-such that a coherent unit system would also be founded on four base units, three of mechanical and one of electrical nature, in agreement with the 1901 proposal of Giorgi.

In addition scientists became more and more convinced that it would be logical to introduce one more change in Maxwell's set of equations for electricity, i.e., to introduce numerical factors $4 \pi$ in various places, where 
they would logically occur, and to drop them from other places (so-called "rationalization"). As a result of the coherencc of the unit system, this docs not change the formal expressions for the units, but it does change the sizc of sone of the corresponding standards. In fact it also modifies the value of the vacuum permeability into $4 \pi \cdot 10^{-7}$ MKS-units of force per ampere squared. So at present the four-dimensional set of equations for electricity is always written in the "rationalized" form.

After long discussions in various international organizations the International Electrotechnical Commission, at its meeting in Scheveningen in 1935, adopted the Giorgi proposal, leaving still open, however, the choice of the fourth quantity and unit of electrical nature on which the system should be based.

In this connection it is important to note that Giorgi, supported by various other scientists, was in fact in favor of choosing this fourth electric unit to be the international ohm, defined by an independent primary standard. Others, however, favored one of the practical units defined as mentioned above on an "absolute" basis. The choice was left open in order to obtain the opinion of the International Committee for Weights and Measures and that of the S.U.N. Commission for Symbols, Units and Nomenclature of the International Union of Pure and Applied Physics - presided over by Sir Richard Glazebrook - which commission in the past had already made inportant contributions to the formulation of the new ideas.

What made the discussions and decisions so difficult at that time. were the conceptionally very different points of view which various scientists had about the philosophy of setting up a unit system: on one hand there was the metrological point of view, which was trying to define a measuring standard for every quantity and to establish primary standards for a certain set of base units which were metrologically independent. From this metrological point of view the original Giorgi proposal with an independent base unit for resistance represented by a primary standard, was the simplest solution. Defining electric quantities by giving the permeability of vacuum the numerical value $4 \pi \times 10^{-7}$ seemed to be strange to the usual metrological procedures. It appears to be similar to defining the unit of mass in terms of the metre by giving the density of water, at the temperature of maximum density, the value of $10^{3} \mathrm{~kg} / \mathrm{m}^{3}$ by definition, a principle which was originally aimed at in the metric system, but which was given up later in view of the metrological praxis, which required standards for both length and mass.

On the other hand there was the systematic point of view, which looked primarily at the algebraic rules, which any unit system-coherent with a system of equations between physical quantities-has to satisfy. This required a system with a fourth independent electric quantity and unit - independent from the systematic or algebraic point of view. The reasons are then essentially the arguments, partly theoretical or algebraic and partly 
more philosophical, which were given above in favor of the four-dimensional system.

In the case under consideration the systematic point of view prevailed. The International Committee decided in 1935 that:

(1) the permeability of vacuum, in the rationalized form of writing the equations, was defined to be $4 \pi \times 10^{-7}$ newton per ampere squared, where the newton was introduced as a new name for the MKS-unit of force.

(2) a definition of the ampere in terms of the force bet ween two parallel conductors was considered to be the most appropriate basis for deriving all the other practical electric units.

However, for the transition from the abandoned "international" units to the "absolutely" defined electric units one now had to make available accurate values for the conversion factors. The final decision on these conversion factors was interrupted by World War II. However, in 1946 the International Committee, authorized by the General Conference in 1933, formally confirmed its previous decisions, the date for the introduction of the "absolute" practical electric units being decided to be January 1, 1948.

\section{The International System of Units}

The general tendency, existing after World War II, to furtler intensify international collaboration and to review the existing situation with the aim of promoting everything which would advance better international understanding in particular in science and technology and in international industrial and trade relations, had also a definite influence on the development of a generally accepted international unit system. There still existed a large diversity of systems: in France the metre-tonne-seconde (M.T.S.) system had been introduced legally in 1919; in technical circles a technical metric system, the metre-kilogram-force-second systenı, was generally used with the confusing name "kilogram" for a base unit of force instead of mass; physicists used the CGS system; and in England and the U.S.A. similar systems based on the foot, pound, and second had been used for centuries.

The International Union of Pure and Applied Physics and its S.U.N. Commission for Symbols, Units and Nomenclature had expressed the need for the international adoption of an "international practical system of units" for international communication, to be based on the metre, the second, the kilogram and an electric unit of the absolute practical system. A similar request was made by the French government. This resulted in an international inquiry made by the International Committee-on the request of the 9th General Conference in 1948-in scientific and technical circles and in institutions charged with education and teaching. On the basis of the information obtained, the 10th General Conference decided in 1954 that such an international practical system should be based on six 
base units, i.e., the metre, kilogram, second, ampere, kelvin, ${ }^{3}$ and candela. The choice of the first set of four base units agreed with the MKSA-system already approved in 1948. The introduction of the kelvin and the candela may require some special attention.

So far the physical phenomena for which a unit system was designed were of purely mechanical, electrical or magnetic nature, such that their description in terms of physical quantities could be based entirely on the four base quantities: length, time, mass, and electric current. However, for the description of thermal phenomena a new base quantity, the thermodynamic temperature, has to be introduced both for systematic and for metrological reasons. The ratio of the thermodynamic temperatures of two systems is determined by thermodynamics-using a Carnot cycle-in terms of the ratio of two amounts of heat (or energy). In agreement with the procedure followed in defining standards for other base quantities it is then sufficient to attach - by definition - a certain numerical value to the thermodynamic temperature of one particular physical state. In 1948 the General Conference had selected this physical state to be the triple point of water and in 1954 the corresponding thermodynamic temperature was defined to be 273.16 kelvins $^{3}$.

If we now turn to the description of the phenomena of light it appears that here three base quantities are sufficient: length and time and one physical quantity of luminous origin for which the luminous intensity has been chosen. The candela, the unit of luminous intensity, is then defined as the luminous intensity in the perpendicular direction of a particular area of a blackbody at a certain specified temperature. This definition was approved by the General Conference in 1948 and was put in its final form in 1968.

In 1960 the 11th General Conference gave the name "International System of Units" to the unit system based on the six base quantities and units already introduced in 1954 . For all other quantities the corresponding derived units can be expressed in the base units, although in many cases special symbols are given. Only for the two quantities plane angle and solid angle was it left undecided by the General Conference-in view of the many different existing opinions in this field-whether these have to be considered as base quantities or as derived quantities. The corresponding units, the radian and steradian, were placed in a separate group with the name "supplementary units" and may be regarded either as base units or as derived units. The base units and the derived units togrether with these supplementary units, were called SI Units, where "SI" is the generally adopted abbreviation for International System. This set of SI units forms a coherent system of units in the sense explained in the previous sections of this article: the system is directly related to the whole set of algebraic equations on which physics, chemistry, and technology at present is based.

"Still called the degree Kelvin at that time. 
The system includes also a set of symbols for prefixes to be used to form decimal multiples and submultiples of the SI units.

It has already been stated that the whole structure of the International System of Units is intimately related with, and forms part of, the whole mathematical description of the physical phenomena, on which modern sciences are built. But then it must also be understood that such a system of units cannot be adopted for eternity: as science is developing, so is the International System of Units. One must expect that from time to time necessary improvements or changes have to be introduced in order to keep the system up to date with developing science.

When the system was adopted it was already evident, for instance, that the quantity mass, although it may be an appropriate concept in mechanics, is entirely unsuitable for use in chemistry where the molecular structure and in particular the number of molecules in a system is much more relevant than its total mass. For that reason the concept "amount of substance" has been introduced in chemistry as a useful and necessary base quantity with the base unit mole, which by definition is the amount of substance of 12 grams of carbon-12. In 1971 the General Conference added this base quantity and its unit as such to the International System of Units.

The International System has had a considerable impact on science and technology, in the international relations between countries and civil life: in education and teaching at the primary, secondary, and university levels. Of course the gradual development of this system has not eliminated at once all other systems, neither has the great diversity of units not fitting into the system suddenly disappeared. Some of these units had to be retained for general use, but others have to disappear in the course of time. General rules and recommendations have been given in the brochure "The International System of Units" published by the International Bureau and edited for the International Committee by its Consultative Committee for Units. In this brochure one finds the full definitions of the seven base units of the International System together with further explanations concerning their practical realization. The logical structure of the International System - with its SI units and their decimal multiples and submultiples - is presented together with various rules adopted by the General Conference about the symbolic representation, and additional recommendations of the International Organization for Standardization (ISO). Both of these are essential for the incorporation of the International System into the whole mathematical structure which describes our present day knowledge in the field of the exact sciences and technology. I would like to close this contribution by expressing the hope that the very fruitful cooperation which exists between various international organizations will continue with the aim of further improving the International System as an efficient tool in the field of science and technology and as an essential link between the peoples in a growing world of ever increasing complexity. 

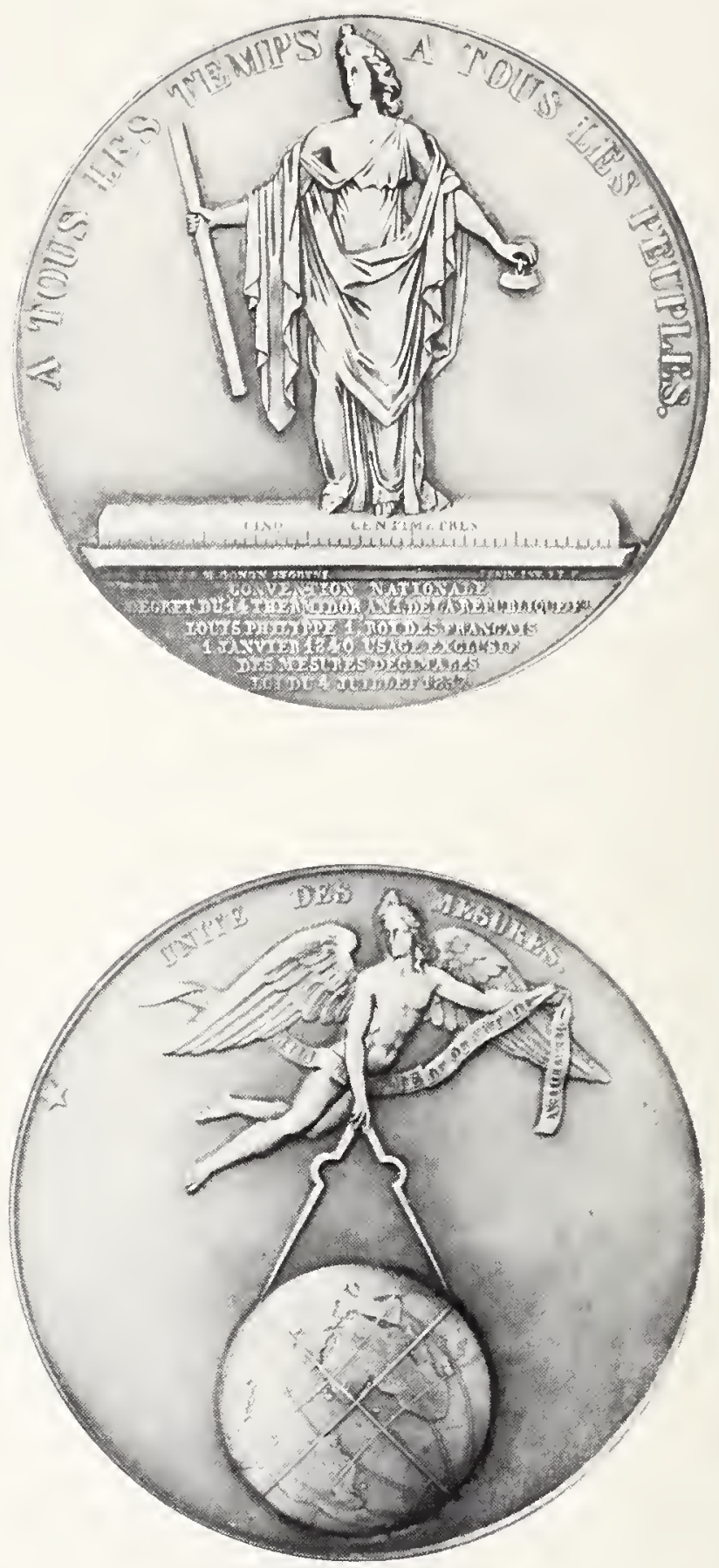

Metric System medal struck in 1840 after a 1799 design. 


\section{CHAPTER I}

\section{HISTORICAL INTRODUCTION}

\section{THE METRE CONVENTION AND ITS ORGANS}

A century has passed since the Diplomatic Conference on the Metre was convened in Paris on March 1, 1875, at the invitation of the French Government [1]. At the closing session of that Conference, May 20, 1875, the plenipotentiaries of 17 of the 20 countries represented signed the treaty known by the name, Convention of the Metre. This Convention and its appended Regulations sanctioned the birth of the International Bureau of Weights and Measures (BIPM), ${ }^{1}$ a scientific and permanent agency supported at the common expense of the signatory countries; its location was set in France, the cradle of the Metric System. The BIPM was placed under the authority of a diplomatic conference, the General Conference on Weights and Measures (CGPM), and of a committee of scientific experts, the International Committee for Weights and Measures (CIPM). This agency has for its goal the assurance of the "international unification and development of the Metric System."

The BIPM must, for its part, "establish new metric standards, conserve the international prototypes, and carry out the comparisons necessary to assure the uniformity of measures througlout the world."

The accomplishments prior to the fiftieth anniversary of the creation of the BIPM have been described in the volume on that occasion published in 1927 [2]; the origins of the Metric System and of the Convention of the Metre, and the history of the Pavillon de Breteuil have an important place therein along with the work of the BIPM during the first half century of its existence. We shall repeat only the main topics of this past.

The Metric System was born from the desire to put an end to the chaos which reigned in France in the field of measurements (decree of May 8 , 1790). Its founders, however, immediately set themselves a more noble aim: to base the measures of the new system on a "universal natural unit" which belonged to no one and which could be adopted by all countries

\footnotetext{
${ }^{1}$ Appendix 7 gives the list and meaning of the abbreviations used in this brok.
} 


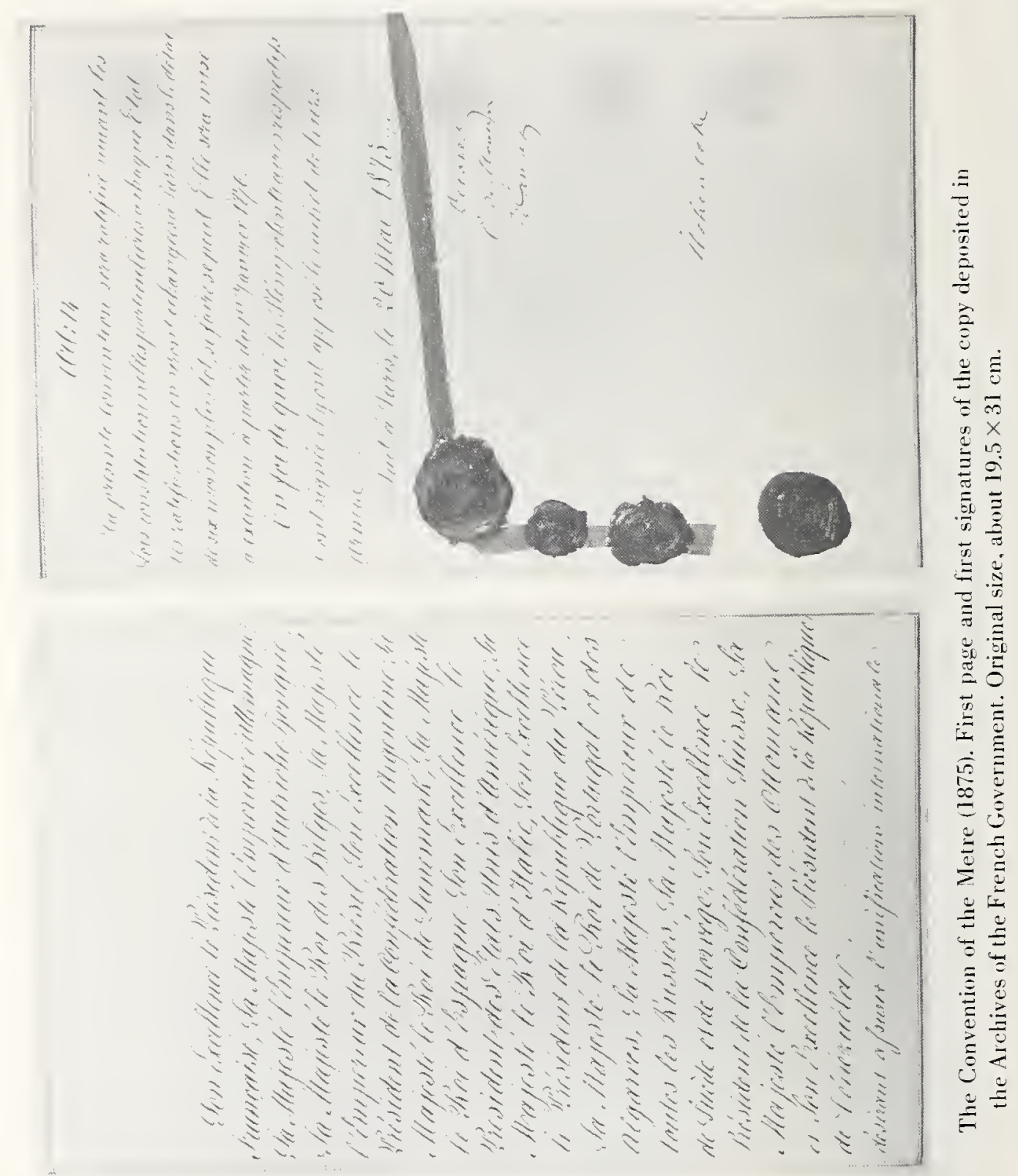


without problems of national pride. Their choice thus fell on a decimal system whose unit of length, the metre, was to be the ten-millionth part of a quarter of the terrestrial meridian, and the unit of mass to be that of a cubic decimetre of water.

This new system was legalized in France on April 7, 1795; the law of December 10, 1799, fixed the values of the metre and kilogram by adopting the prototype platinum standards known by the names Metre of the Archives and Kilogram of the Archives.

After slow and difficult beginnings, hobbled by contradictory decisions $(1800,1812,1816)$, the law of July 4, 1837, finally made the Metric System compulsory in France starting on January 1, 1840.

Gradually learned of in foreign countries, appreciated for its simplicity, its logical structure and universal applicability, the Metric System began to spread through Europe and the world. On the occasion of international expositions held between 1851 and 1867, "when man found himself in the presence of the immense variety of products sent from all parts of the world, and whose values as well as quantities were described in all kinds of measurement standards," the need for unification struck all clearsighted minds. A "Committee for weights, measures, and currencies," set up on the occasion of the 1867 Paris Exposition, announced itself in favor of universal adoption of the Metric System.

The momentum of opinion was launched and events rushed forward after the resolutions and recommendations adopted between 1867 and 1869 by the International Association for Geodesy, the Bureau of Longitude (Paris), and the Academies of Science of Saint Petersburg and Paris. The International Commission of the Metre $(1870,1872)$ took the first practical

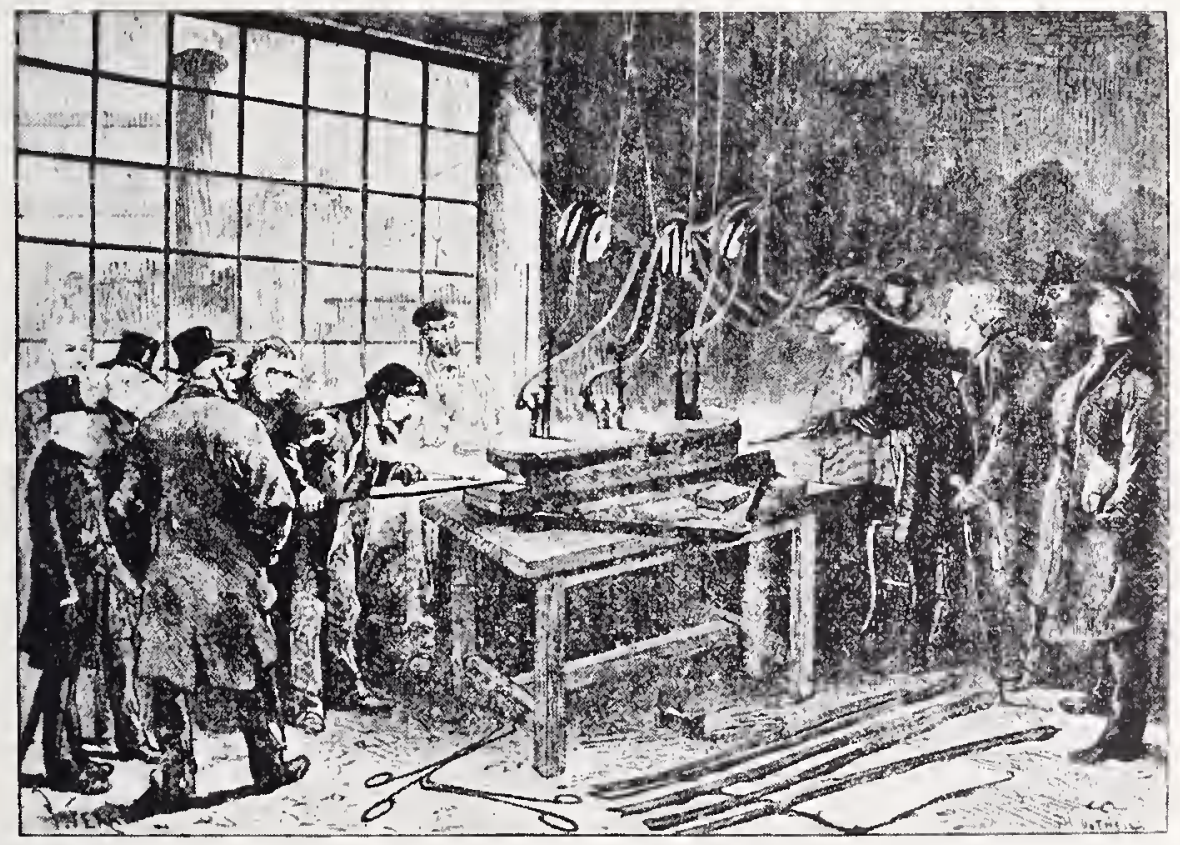

Casting the platinum in idium alloy called "1874 Alloy," at the Paris Conservatory of Arts and Crafts. (L'Illustration, May 16, 1874). 
steps toward the internationalization of the Metric System. The permanent working committees and the French Section of this Commission constructed new metric standards, derived from the original standards of the Archives, which were to be distributed throughout the world.

All the preparatory tasks and deliberations culminated in 1875 in the signing of the Convention of the Metre (app. 1, p. 225) and in the founding of the International Bureau of Weights and Measures.

Since 1875, the organs of the Metre Convention have not been subjected to change. However, by reason of the increasing complexity of scientific questions related to metrology, the CIPM established several Consultative Committees, starting in 1927. The relations among these organs, the member States, the specialized international organizations, and the national laboratories are shown below.

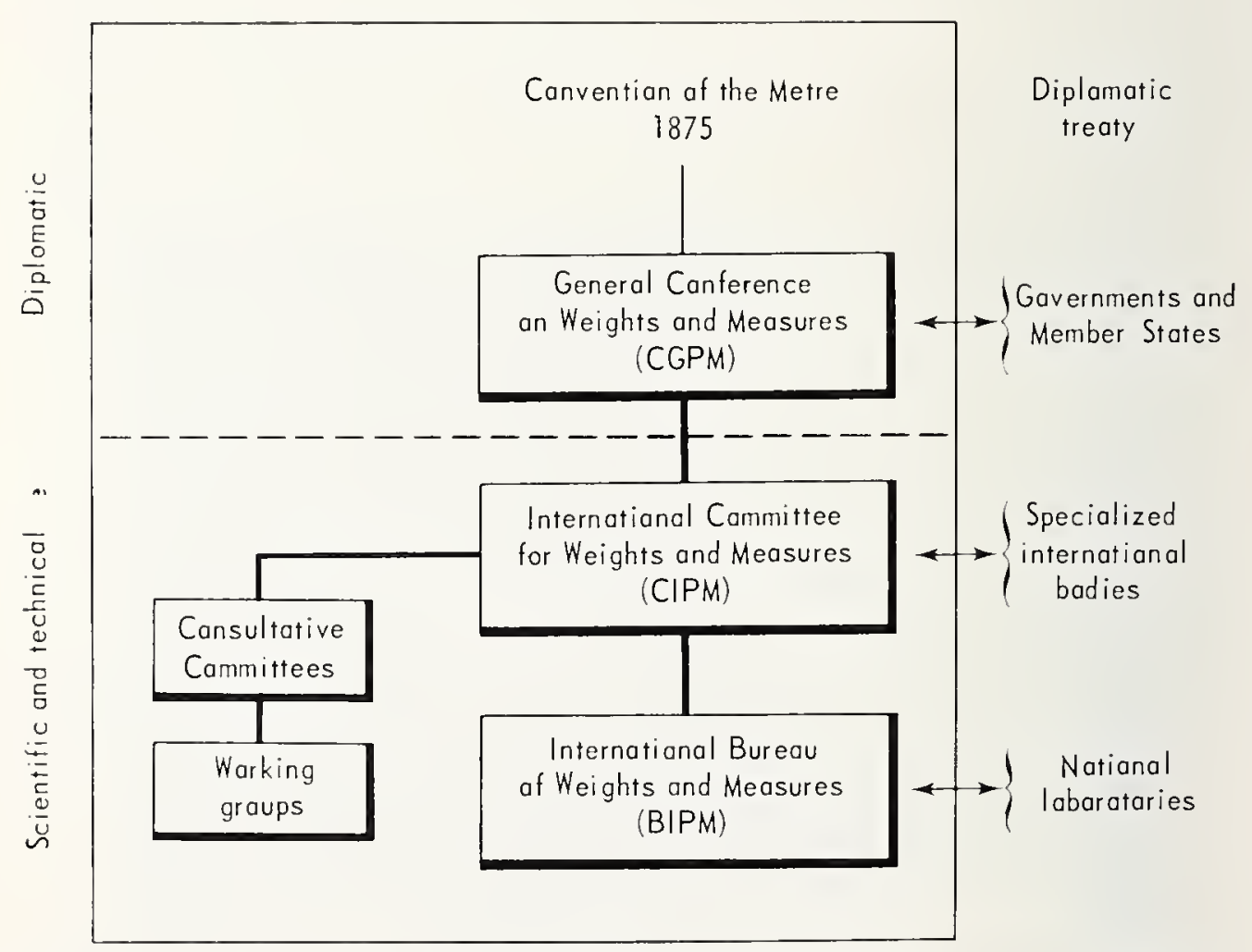

The Metre Convention Organs. In 1975, there are seven Consultative Committees: Electricity, Photometry and Radionetry. Thermometry, Definition of the Metre, Definition of the Second, Ionizing Radiation, Units. 
The General Conference on Weights and Measures, an intergovernmental conference comprising delegates of member States of the Metre Convention, ${ }^{2}$ is the supreme authority which controls the administration of the BIPM; it has for its primary mission:

- to discuss and initiate the necessary measures to assure the propagation and development of the International System of Units (SI), the modern form of the Metric System;

- to ratify the results of new fundamental metrological determinations and to adopt various scientific resolutions of international importance;

- to adopt important decisions concerning the organization and development of the BIPM.

From 1889 to 1971 , the CGPM met 14 times. The 15th CGPM coincides with the centennial of the Convention of the Metre.

The International Committee for Weights and Measures is charged with preparing and executing the decisions of the CGPM. It directly supervises the operation of the BIPM and oversees its work.

Originally composed of 14 members, a number raised to 18 in 1921, the CIPM assembles men of science and cminent metrologists of all different nationalities (app. 3, p. 233). Elected by the CGPM, these members sit in the CIPM in a personal capacity and they are not in any way official representatives of their countries. The CIPM met for the first time in April 1875; it held its 63d meeting in 1974, these meetings having been annual or biennial at various times. Between meetings of the CIPM, its executive committee-comprising a president, a vice-president (position created in 1954), and a secretary-examine current business, keep the CIPM informed on important questions, and prepare the meetings. The director of the BIPM participates ex officio in the meetings of the CIPM and in the meetings of its executive committee.

Each of the Consultative Committees - occasionally helped by "Working Groups" - assembles a small number of representatives of the best laboratories in the research and measmements peculiar to a particular field of metrology.

These Consultative Committees, currently seven in number, have the mission of advising the CIPM on questions of a scientific nature which it submits to their examination: orientation of the work of the BIPM, coordination of this work with that of the national metrological laboratories. organization and promotion of international undertakings, decisions which the CIPM is led to take directly or to submit to approval by the CGPM.

2 As of January 1, 1975.43 States were members of this Convention (see app. 2. p. 231, for the list of these States and the dates of accession). 
The International Bureau of Weights and Measures, organ for execut ing the decisions of the CGPM and the CIPM, is a permanent laboratory and the world center of scientific metrology whose progress, particularly spectacular in our time, is intimately linked to the development of scientific discoveries, industrial technology, and international comparisons.

The primary mission of the BIPM is:

- to establish the basic standards and scales of the principal physical quantities, and to maint ain international prototypes:

- to carry out comparisons of national and international standards;

- to assure coordination of the corresponding techniques of measurement:

- to carry out and coordinate relative determinations of fundamental physical constants.

Functioning of the BIPM is assured by an annual appropriation voted by the CGPM. Since 1960, this appropriation has been divided among the member States of the Metre Convention according to a scale based on the factors used by the United Nations; the contributions of the States can not. however, be greater than 10 percent, nor less than 0.5 percent, of the total appropriation. From an amount of 100000 gold francs in 1878, the annual budget of the BIPM is now some 4180000 gold francs for 1975 .

The BIPM has a staff which can be of all nationalities; its director, named by the CIPM, must be of a nationality different from those of the CIPM officers. This staff, scientific, administrative, technical, and service, did not surpass some 10 persons formerly; it consists of some 50 persons in 1975.

Like any international organization, the BIPM has, at certain times in it s existence, known financial difficulties: these have becn overcome, thanks mainly to subsidies and various gifts, and to generous offers of materials and scientific instruments. The periods of the two world wars were particularly difficult, but very fortunately the BIPM was able to survive then with neither the international metric prototypes nor the measuring apparatus suffering any damage.

A neutral and autonomous organ, the BIPM is not dependent on any of the existing intergovernmental organizations ${ }^{3}$ and is not affiliated with any international union or association. It has been recognized in France as an establishment of public usefulness by the decree of October 28. 1876. On April 25, 1969, an Accord was concluded between the French Government and the CIPM relative to the grounds of the BIPM and to its privileges and immunities in French territory (Decree No. 70-820 of September 9, 1970).

\footnotetext{
${ }^{3}$ Agreements on information and mutual relations have, however, been concluded with the United Nations Educational. Scientific and Cultural Organization (UNESCO) (1948), the International Agency for Atomic Energy $(1961,1967)$, the European Atomic Community (EURATOM) growing out of the Commission of the European Communities $(1965,1967)$, and the International Bureau of Legal Metrology (1970).
} 


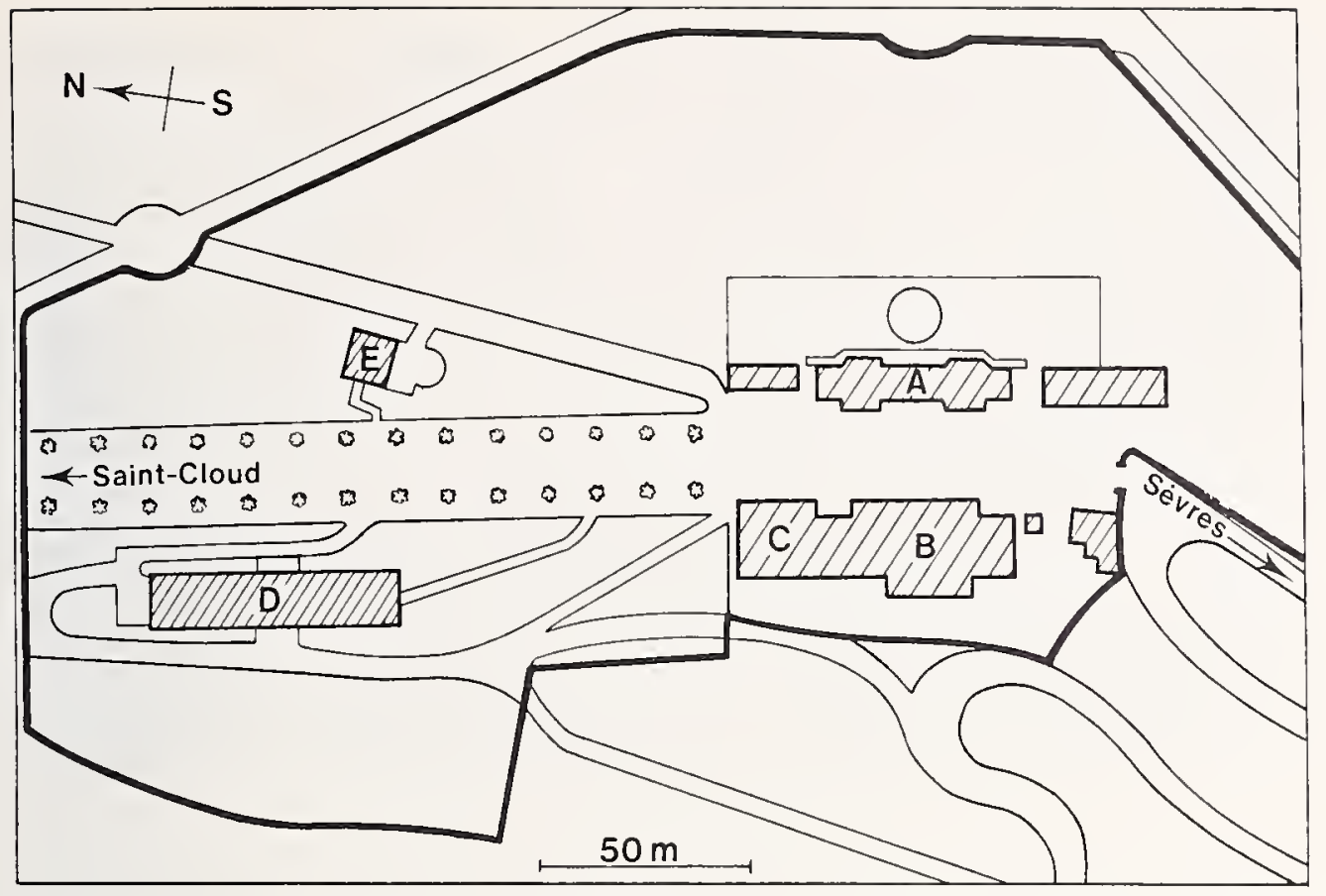

Overall plan of the Pavillon de Breteuil and the lahoratories of the International Bureau of Weights and Measures in 1975. A: Pavillon de Breteuil: B: laboratories (1878): C: extension of laboratories (1929): D.E: laboratories for ionizing radiations (1964).

These grounds are situated in the Park of Saint-Cloud, near Paris. The French Government graciously placed at the disposal of the CIPM, April 22, 1876, an estate of $25153 \mathrm{~m}^{2}$ in area, ${ }^{4}$ where is found the Pavillon de Breteuil-name under which the BIPM is also known worldwide.

The Pavillon de Breteuil proper is a building with a historic past; built in 1743 on the site of the former Trianon of Saint-Cloud (17th century) it was greatly damaged in 1870-1871. The CIPM restored it and constructed a new building (1878) for the laboratories: extension of the activities of the BIPM required enlarging the first laboratories in 1929 and the construction of new buildings in 1964.

Such are, briefly summarized, the history and essential characteristics of the different organs born of the Metre Convention. In close collaboration with the principal national metrology laboratories, these organs have for a century unflinchingly fulfilled the mission assigned to them in 1875; their permanence reflects that of the Metric System. which will itself soon celebrate its bicentennial.

\footnotetext{
${ }^{4}$ Two extensions have increased this area to $43517 \mathrm{~m}$.
} 


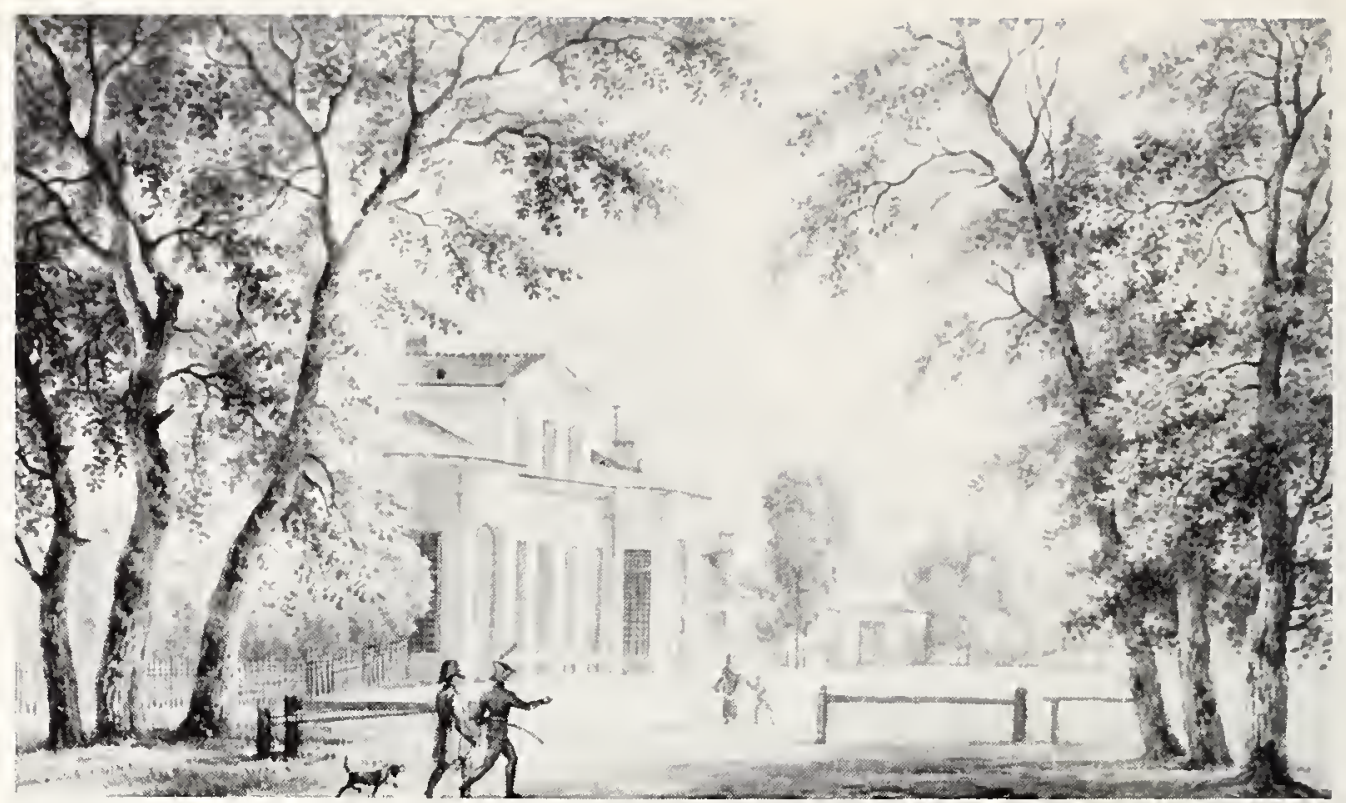

The Pavillon de Breteuil, then called Pavillon d'Italie, at the beginning of the 19th century. (Lavis de Langlacé, 1807. Manufacture Nationale de Porcelaine de Sèvres).

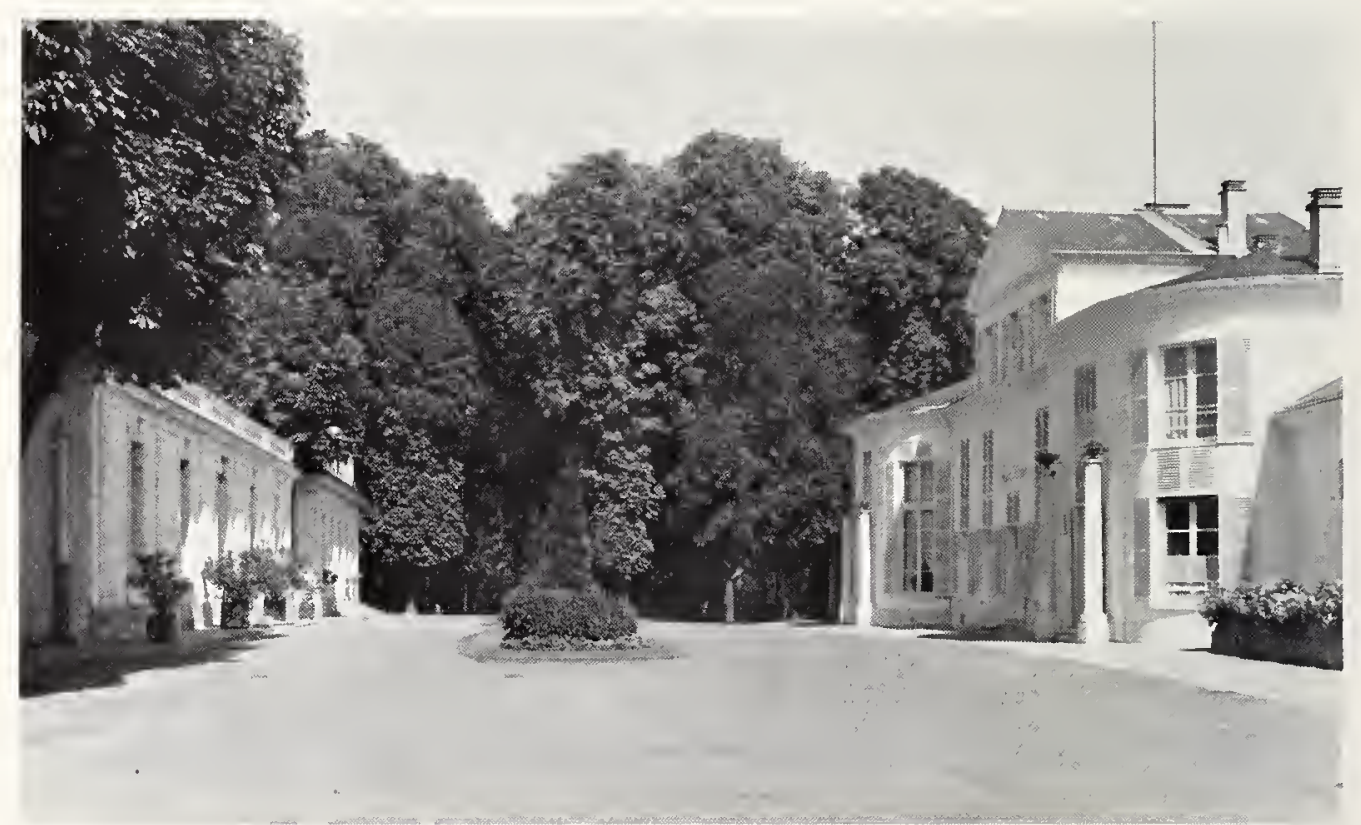

The Pavillon de Breteuil. seat of the International Bureau of Weights and Measures (geographical courdinates: $48^{\circ} 50^{\prime} \mathrm{N}, 2^{\circ} 13^{\prime} \mathrm{E}$, altitule 66 metres). on the right: offices, conference $r o m$ : on the left: laboratories built in 1878 and in 1929 (the laboratories built in 1964. located at the rear on both sides of the central lane, are not visible in this photograph). 


\section{A Century of Metrology $y^{5}$}

\section{5-1925}

The initial mission of the BIPM was to participate in the preparation and determination of the values of the new metric prototypes of platinumiridium destined to be the international and national prototypes. The results of this important task [3] were presented to the 1st CGPM (September 1889); the CGPM ratified the definitions of the units of length and mass based on the international Metre and Kilogram ${ }^{6}$ as well as the equality of some 30 Metres and 40 Kilograms which were distributed by lot among the States.

At the time of founding the BIPM, it was thought that after these first basic determinations, its activity would be reduced to periodic verification of the national st andards.

It was very quickly seen that this activity implied numerous permanent auxiliary studies. It is in fact necessary to be able to determine the submultiples and multiples of the units with precision, if only to evaluate accurately the differences among the standards to be verified. It is also necessary to study the phenomena which affect the measurements, for example, dilatation in the case of length measurements, or air pressure in the case of mass measurements; this requires the measurement of numerous physical quantities: temperature, volume, density, pressure, etc., which moreover have considerable practical importance. It is still necessary to perfect methods capable of improving the comparisons and to research better secondary standards for routine use; the use of material standards in fact always carries a risk of deterioration which should be reduced to the strict minimum for primary standards. Finally, there is the application of new scientific discoveries leading to better definitions and units, and the BIPM should be in a position to bring them into play.

The BIPM was thus led, apart from the verification of national standards which today still constitutes its principal activity, to carrying out work of which certain parts have strongly marked the history of metrology [1].

The measurements of mass have given rise to various tasks: studies of the balance, methods of calibrating sets of weights, determination of the values of British standards in metric units, effect of the sublimation of

\footnotetext{
${ }^{5}$ See appendix 6, p. 245, for the list of publications in which are listed the activities of the organs of the Metre Convention since 1875.

${ }^{6}$ The international Metre and Kilogram were placed, with their verifications, in the lower vault of the Pavillon de Breteuil on September 28. 1889. The three keys needed to open the vault for the metric prototypes were given to the director of the BIPM. the president of the CIPM, and a representative of the National Archives in Paris.
} 


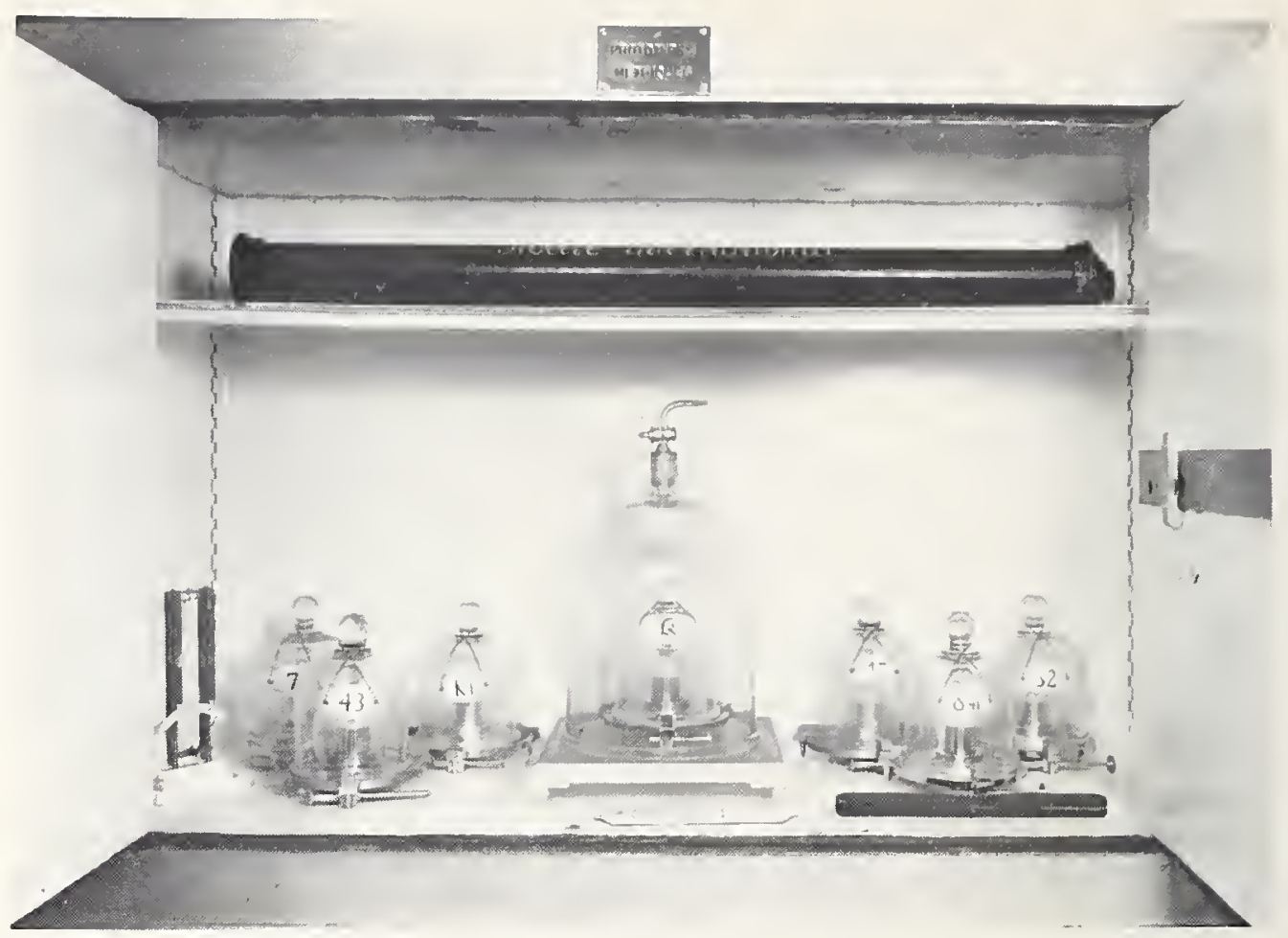

Vault for the metric prototypes, above: the International Metre of 1889 in its metal case; below; the International Kilogram 返, under its three glass domes, and its six check standards. On the lower level are seen two thermometers, one of which is a maximumminimum, and on the left a hair hygrometer

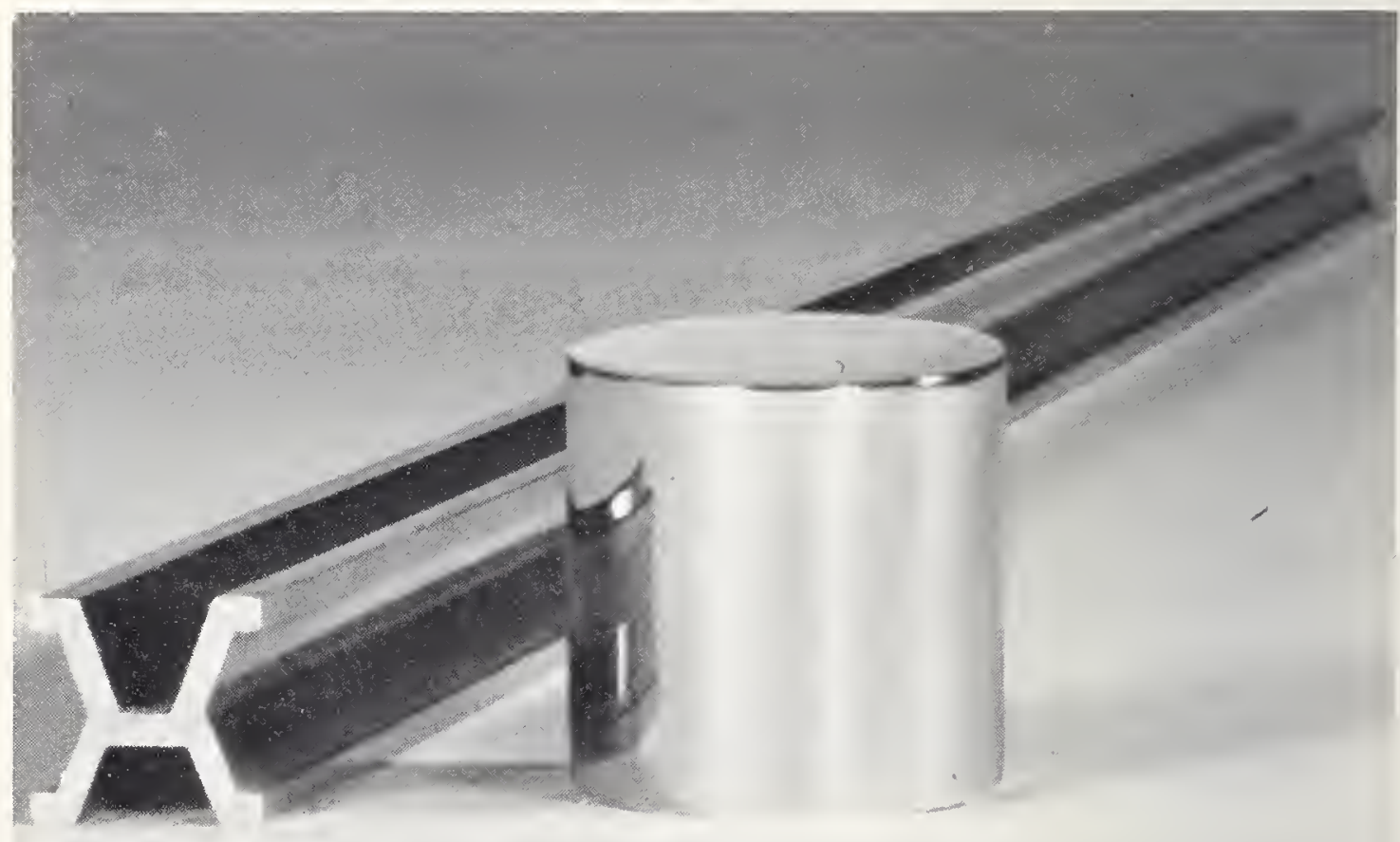

The Internatunal Netre and the International Kilogram of 1889 (life-size front view). 
platinum-iridium on the maintenance of mass standards, determination of the density of air, etc. The density of water, whence derive most volume measurements, was deternined between 1895 and 1905 by three different methods: the result of this important work led to the acceptance for the mass of 1 cubic decimetre of pure water, with the air removed, at $4{ }^{\circ} \mathrm{C}$ under standard atmospheric pressure, the value $0.999972 \mathrm{~kg}$, that is 1.000 $028 \mathrm{dm}^{3}$ for the volume of $1 \mathrm{~kg}$ of water, ${ }^{7}$ this result is still valid in 1975 .

Studies on measurement of length were not less numerous: determination of the coefficient of expansion of the platinum-iridium alloy (comparator method and the Fizeau interferometric method), sharpening up some methods of calibrating divided scales, measurement of geodetic base lines by means of invar wires and tapes, and calibration of these, studies of standards with flat and spherical ends, comparison of non-metric units (toise, yard, etc.) with the metre.

We investigated materials less costly than platinum iridium suitable for making standards; the study of the expansion of nickel steels led to the discovery of some remarkable properties of several alloys, notably invar of very low expansion, elinvar with a zero thermoelastic coefficient, platinite of expansion in the neighborhood of that of platinum and capable of being sealed to glass, nonmagnetic baros or nichrome, alloys of 42 or 58 percent nickel which have an expansion close to that of common glasses or of steel. All these alloys find application not only in metrology, but also in industry. Begun in 1896 on the initiative of J.-R. Benoit, these researches are associated with the name of Ch.-Ed. Guillaume, for whom they won the 1920 Nobel Prize in physics.

The interferometric measurements allowed establishing a link between the unit of length, embodied in the international Metre, and the length of light waves. Around 1890, A. A. Michelson (University of Chicago) proposed to substitute a wavelength for the platinum-iridium prototype; invited to come to the BIPM with his interferometer to apply his measurement methods, he thus established in 1892-1893, in collaboration with J.-R. Benoit, the ratio of the wavelength of the cadmium red line to the international Metre. In 1906, J.-R. Benoît, Ch. Fabry, and A. Perot made a similar determination with new procedures (Fabry-Perot etalons) confirming, with a better accuracy, the result given by Michelson 15 years earlier. The value of this wavelength, which served until 1961 for defining the ăngström, was adopted in 1927 as a standard for spectroscopy and for interferometric measurements of length.

\footnotetext{
T This difference of 28 parts per million represents the approximation with which the Kilogram of the Archives had been realized in 1799 with respert to its initial definition. This result, part of which must undoubtedly be attributed to luck. is remarkable if we consider the available means and the state of science at that epoch. The kilogram is found, in fact, to be represented by the mass of a cube of water which would have a thickness of not exactly 1 dm. but $1.000009 \mathrm{dm}$, only $0.9 \mu \mathrm{m}$ greater!
} 
Other work used applications of interferometry: study of graduated scales, measurement of industrial gages with flat and spherical ends, measurement of quartz (rock-crystal) etalons of 10 to $100 \mathrm{~mm}$ length for use as metric unit references, study of radiations used in metrology, determination of the index of refraction of air, etc.

In the field of thermometry, the work of J. Pernet and P. Chappuis on the gas thermometer led the CIPM to adopt the scale of the hydrogen thermometer as the "standard scale" of temperature in 1887, the first step toward the eventual adoption of the thermodynamic scale. Systematic studies of the mercury thermometer by Ch.-Ed. Guillaume allowed making this instrument suitable for the measurement of temperature to a few thousandths of a degree in a well-defined scale. A consequence was that all the national metre prototypes distributed in 1889 were accompanied by two mercury thermometers of hard glass, with correction tables to convert their readings to the hydrogen-thermometer scale.

In 1897-1898, J. A. Harker (Kew Observatory) and P. Chappuis carried out a comparison of the platinum resistance thermometer with the hydrogen thermometer; this was a prelude to the establishment of a practical temperature scale which was adopted 30 years later.

The acceleration of gravity, an important physical constant which is involved in the realization of several units (units of force, pressure, power; electric units, etc.), was determined at the Pavillon de Breteuil by Defforges in 1888, by the method of the reversible pendulum. The result obtained (referred to sea level at $45^{\circ}$ latitude) led the $3 \mathrm{~d}$ CGPM (1901) to adopt the conventional value $9.80665 \mathrm{~m} / \mathrm{s}^{2}$ as the "standard value" of $g$ for use in the reduction to standard gravity of observations made at an arbitrary location.

This brief reminder shows how fertile was the activity of the BIPM during the first 50 years of its existence; although limited to the quantities mass and length, and to metrological studies related to the measurement of these quantities, this activity was essential to the development of scientific and industrial metrology.

\section{5-1975}

Three salient facts characterize the second half-century of existence of the organs of the Metre Convention: 1st, the extension of the activities of the International Bureau of Weights and Measures to units and standards in the fields of electricity, photometry, and ionizing radiation; $2 \mathrm{~d}$, the greater and greater participation of the International Committee of 
Weights and Measures in international cooperation in matters of scientific metrology, extending to standards of time and frequency; $3 \mathrm{~d}$, the revitalization of metrological methods, related to developments in several branches of physics (electronics, atomic physics, physics of solids, etc.) which have made possible for example, replacing the definition of the length and time units by definitions based on atomic properties. This evolution of the initial mission and fields of activity of the BIPM and CIPM is the natural consequence of the need for more and more precise measurement standards and of the necessity of assuring coordination of worldwide metrological work.

Before presenting the activity of the BIPM during the period 1925-1975 in the following chapters, it seems useful to outline the principal stages of this evolution. These stages have been marked by the creation of various Consultative Committees of the CIPM.

\section{Electricity and Photometry}

The idea of including electricity and photometry in the scope of the BIPM is very old. In deference to the conclusions voted by the "International Conference on Electric Units" (Paris, 1884), a draft of an international convention was prepared by the French Government with an eye to entrusting the BIPM with the construction, verification, and maintenance of standards for electricity and light. This proposal, examined by the CIPM in 1885, was not followed up at the time even though most of the Member States of the Metre Convention had received it favorably.

Later, the question of extending the activities of the BIPM to the electric units was raised on different occasions. ${ }^{8}$ It was nevertheless not until 1921 that the BIPM received from the 6th CGPM the mission of "the establishment and maintenance of standards and references for electric units, and also the comparison of national standards or other precise standards with these." In 1927, the CIPM decided to establish a Consultative Committee for Electricity (CCE), which held its first session in November 1928. After the French Government had granted a small extension ( $\left.285 \mathrm{~m}^{2}\right)$ of the BIPM grounds, new laboratories were constructed in 1929, adjoining the laboratories built in 1878, and the first comparisons of national standards of electric resistance and of electromotive force were carried out at the BIPM in 1932.

Unification of the methods of photometry and the question of the units of light also held the attention of the CIPM which in 1929 requested the CCE for advice on these subjects. The CCE then took the name

\footnotetext{
${ }^{8}$ See a resume of this question in Procès-Verbaux C.I.P.M. 15, p. 169 (1933).
} 
Consultative Committee for Electricity and Photometry, until 1933, at which date the CIPM decided to create a Consultative Committee for Photometry (CCP), distinct from the CCE.

At its first session in June 1937, the CCP requested, in its report to the CIPM, the creation of a photometric laboratory at the BIPM for comparing national standards of intensity and of luminous flux. This laboratory was planned in the basement of the building constructed in 1929, and its essential installations were finished in 1939.

In 1971, the name of the CCP was changed to Consultative Committee for Photometry and Radiometry (CCPR); there is in fact more and more tendency to substitute energetic quantities (radiometry) for visual quantities (photometry); this permits quantitative characterization of infrared and ultraviolet radiation as well as visible radiation.

\section{Thermometry}

In 1913, the 5th CGPM was concerned with the adoption of a thermodynamic scale as a replacement for the standard hydrogen thermometer scale, and with the fixing of a certain number of thermometric reference points suitably chosen for establishing a practical temperature scale. To this end. a meeting of directors of national laboratories interested in the development of thermometric studies had been planned for November 1914; events did not allow this meeting to take place: some 10 years later, the plan for a "Conference on Thermometry" was revived. In 1927, the CIPM was informed that the national laboratories of Germany, the United States of America, and Great Britain had agreed to adopt a common practical temperature scale, as close as possible to the thermodynamic scale, based on fixed points and interpolation procedures. This scale was adopted provisionally by the 7th CGPM (1927) under the name "International Temperature Scale," then in a revised form, but still provisionally, by the 8th CGPM (1933).

The convocation of the International Conference on Thermometry being repeatedly postponed, the USSR Institute of Metrology (1935) and the International Institute of Refrigeration (1937) proposed the creation under the CIPM of a Consultative Committee for Thermometry; the creation of this committee was decided by the CIPM in June 1937.9

The Consultative Committee for Thermometry (CCT) - for some time called the "Consultative Committee for Thermometry and Calorimetry" met for the first time in July 1939, this meeting taking the place of that of the International Conference on Thermometry. Since its creation, the

\footnotetext{
"See the Procès-Verbaux C.I.P.M. 18, p. 96 (1937) for a chronological summary of the events leading to the creation of this Consult ative Committee.
} 
CCT has been concerned mainly with perfecting and extending the "International Practical Temperature Scale"; its work has also led to the adoption of the thermodynamic scale having a single fixed point, with the triple point of water as the fundamental fixed point and with the joule as the unit of heat replacing the calorie.

\section{Definition of the Metre}

The possibility of having a natural standard of length in place of a national standard such as the International Metre has been a constant objective of metrologists. The red line of natural cadmium, which had been proposed, could not, however, fill this role because it is not sufficiently monochromatic and would not permit defining the metre witl a higher precision than that provided by the International Metre.

Also, as soon as it was known how to separate the isotopes of certain elements and thus obtain practically monochromatic visible radiations, the possibility of using these radiations to define the unit of length came into view. The 9th CGPM (1948) consequently invited the large national laboratories and the BIPM to pursue study of these radiations with the goal of possible establishment of a new definition of the metre based on the wavelength of a visible radiation.

To study the advisability of such a change, the CIPM decided in Oc. tober 1952 on the creation of a Consultative Committee for the Definition of the Metre (CCDM), which held its first meeting in September 1953. Work carried out later in the national laboratories and at the BIPM on the metrological qualities of three proposed radiations led the CIPM to recommend, in 1958, the adoption of the wavelength of the orange line of krypton86 as a new standard for the unit of length. On the 14th of October, 1960, the 11th CGPM unanimously adopted the change of the definition of the metre.

Even though, after a reign of 70 years, the 1889 platinum-iridium International Metre preserved at the Pavillon de Breteuil has lost its position as the primary standard, the role of the BIPM has not diminished much; this role remains, to the contrary, essential for assuring uniformity of length measurements throughout the world.

Its initial mission accomplished, the CCDM carried on its activities with three principal objectives: to perfect the 1960 definition of the metre. to recommend better secondary wavelength standards, and to improve the methods and apparatus for measurement of end-standards and line-standards of length. 
The units and standards of time and frequency were outside the responsibility of the organs of the Metre Convention until 1954, the date at which the CIPM was led to consider these questions. At this time, the International Astronomical Union had just proposed a change in the definition of the second and hoped that this definition would receive the international ratification of the CGPM. Under authorization from the CGPM, the CIPM in October 1956 approved the new astronomical definition of the second (the second of ephemeris time) based on the tropical year, a definition which was ratified in October 1960 by the 11th CGPM.

The appearance of "atomic" standards led the CIPM to create a Consultative Committee for the Definition of the Second (CCDS) in October 1956 to study and propose a physical definition of the unit of time. Studies on the unit and scales of time had been, up to then, the province of astronomers; with the new techniques in the field of ultrahigh frequencies put into practice, these studies began to depend also on the competence of physicists.

The CCDS held its first meeting in June 1957. Its work on atomic standards of frequency and of time interval led to the 1964 provisional adoption of the frequency associated with a transition of the cesium-133 atom as the standard of time interval. In 1967, the 13th CGPM definitively adopted a new definition of the second, based on this same frequency, as a replacement for the astronomical definition.

The CIPM and CGPM were subsequently requested to consider a time scale based on the second defined in 1967; an internationally adopted time scale is, in fact, indispensable for temporal synchronization of events in different places. On the advice of the CCDS, the CIPM in October 1970 adopted a definition of "International Atomic Time" (TAI) and, in October 1971, the 14th CGPM authorized the CIPM to conclude necessary arrangements with the International Bureau of Time, the competent agency in the matter, for the realization of the TAI scale. Dissemination of the TAI with the aid of time signals thus permits putting at the disposal of users, not only the time scale, but also the SI unit, the second.

\section{Ionizing Radiation}

The metrology of ionizing radiation comprises measurements of $\mathrm{x}$ rays and $\gamma$ rays, radiations from radionuclides, and neutron measurements. Its importance has increased strongly since 1950. Nevertheless, the idea that the BIPM would play a role in this field is almost as old as the first steps toward a unification of radioactivity measurements, taken in the beginning of the century. It is interesting to trace the principal events which preceded the creation of the BIPM Section on ionizing radiation. 
When Marie Curie constructed the first radium standard in 1911, she undoubtedly envisaged depositing it at the Pavillon de Breteuil. In fact, J. R. Benoit, then director of the BIPM, officially accepted this thin glass tube containing $21.99 \mathrm{mg}$ of anhydrous radium chloride for safekeeping in 1913: this constituted the international radium standard. This standard was replaced in 1939 by 1 of 20 standards fabricated by O. Hönigschmid in 1934; this new standard was removed from the BIPM in May 1940 for transfer into the country; it returned to the BIPM in 1959 and became its permanent property in 1961.

In 1948, the USSR delegation to the 9th CGPM had already proposed that the BIPM organize comparisons of national radium standards with the international standards ${ }^{10}$ and that these latter be conserved at the BIPM. This proposal was again raised at the CIPM in 1952, and in 1956 the CIPM charged a commission with studying a possible extension of the activities of the BIPM to standards of radioactivity. After the report of this commission, the CIPM created the Consultative Committee for Standards of Measurement of Ionizing Radiations (CCEMRI) in October 1958.

At its first meeting in April 1959, the CCEMRI proposed:

"that the scope of the BIPM be extended to the fields of radioactivity and ionizing radiations, and that it be the central agency:

1st, for the definition of the quantities and units,

2nd, for the establishment of international standards for measurement of activity and of ionizing radiations, neutrons included, taking into account the results of national, international, and other laboratories and institutions."

In October 1960, the 11th CGPM approved the action taken by the CIPM, and invited it to organize in the BIPM a Section on measurement standards for ionizing radiations, equipped with a laboratory and necessary scientific personnel.

In its first three meetings (1959, 1960, and 1961), the CCEMRI defined for the BIPM a work program including in particular the organization of international comparisons. The new Section of the BIPM started operating in March 1961.

After a new expansion (18 $\left.079 \mathrm{~m}^{2}\right)$ of the grounds of the Pavillon de Breteuil, granted by the French Government, and after the 11th and 12th CGPM had voted three special contributions, two new buildings were constructed for permanent laboratories of the Section for ionizing radiations; these laboratories were dedicated on September 29, 1964. Since 1969, the CCEMRI has comprised four sections ( $\mathrm{x}$ and $\gamma$ rays, electrons; measurement of radionuclides; neutron measurements; standards of $\alpha$-particle energy) which correspond to current activities of the BIPM Section for ionizing radiations.

${ }^{10}$ There still exists, in addition to the international standards preserved at the BIPM in Sèvres, a replacement standard kept in Vienna (Austria). 


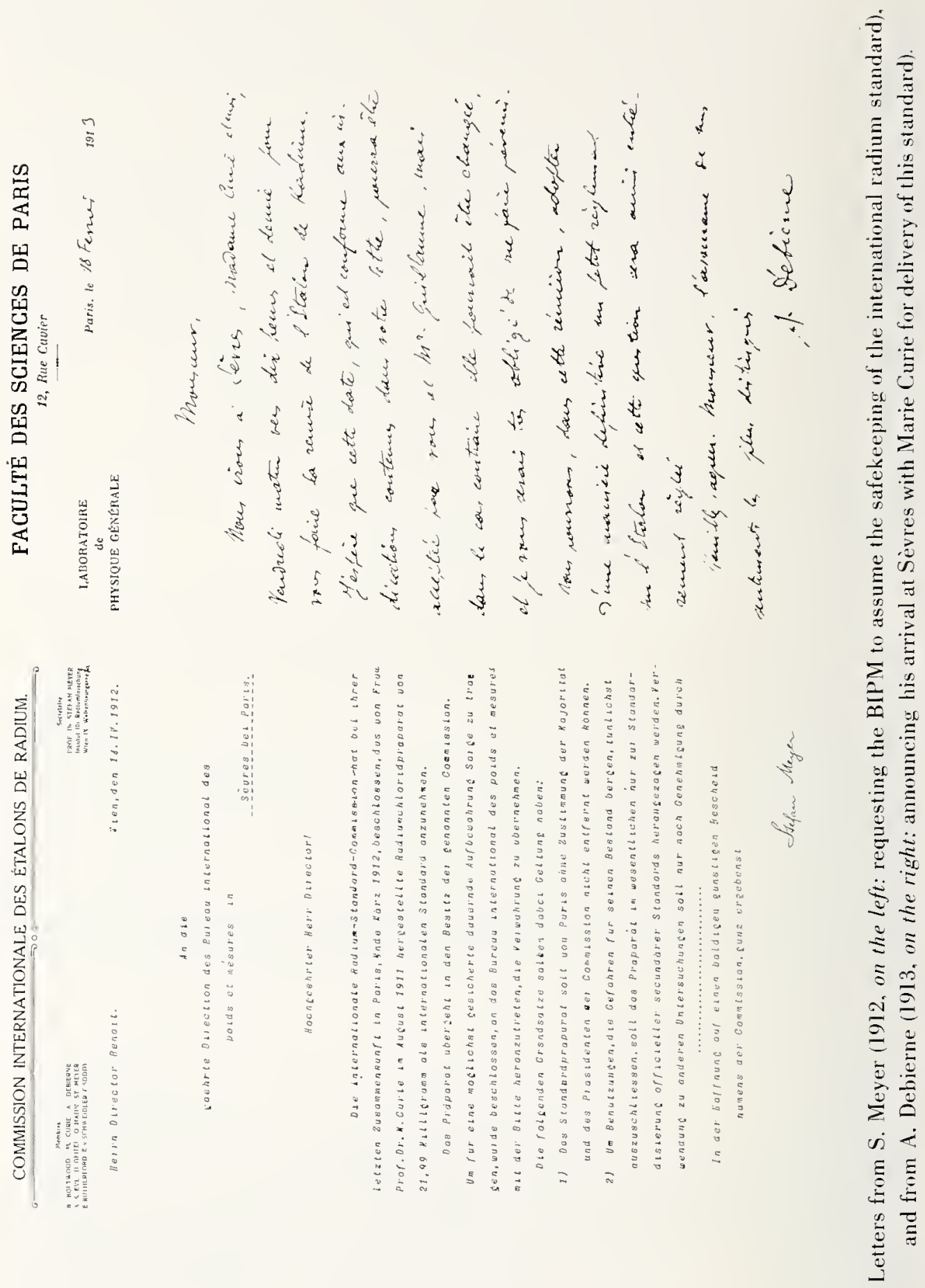




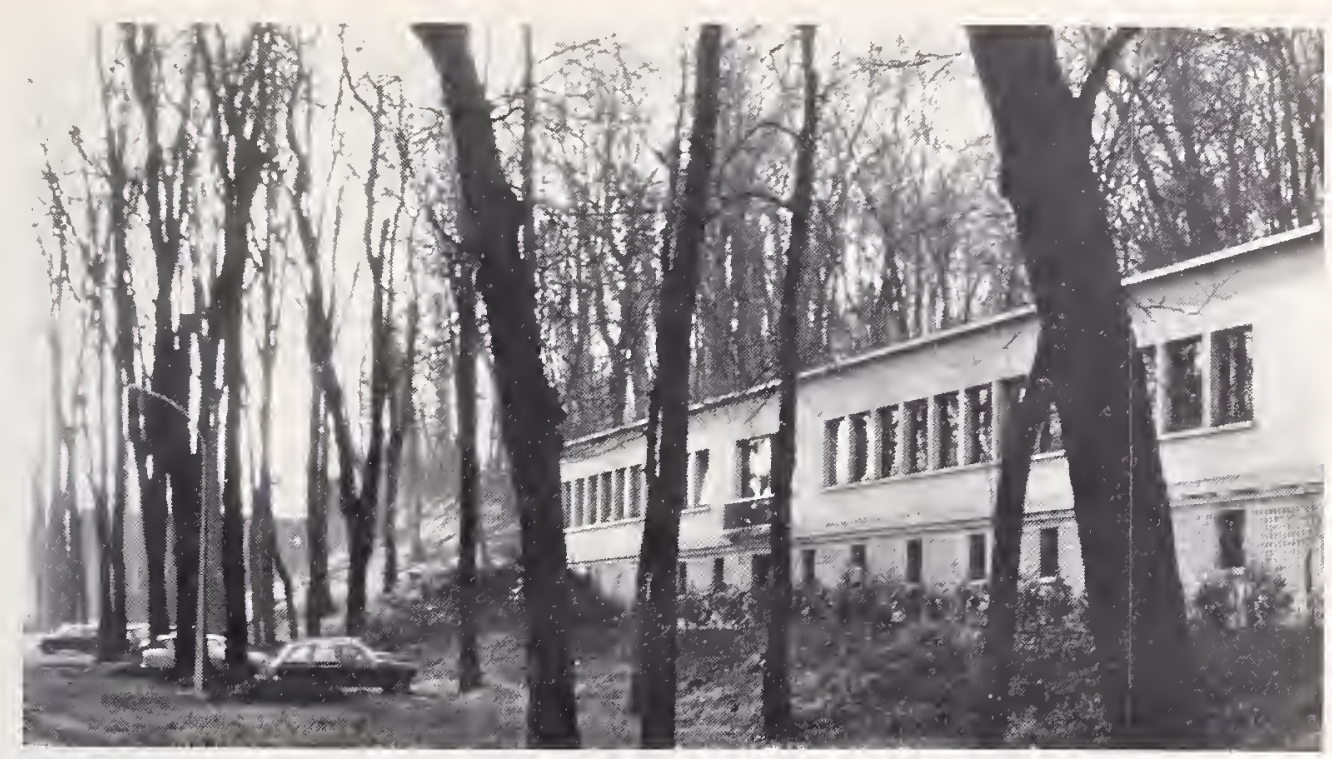

Laboratories of the ionizing radiation section built in 1964 (building D of fig. 5). In the left rear. the 1929 laboratories.

Other international organizations had already been concerned with the metrology of ionizing radiations ahead of the BIPM. In the first place must be mentioned the International Commission on Radiation Units and Measurements (ICRU), founded in 1925, which took a very active part in the creation of the new Section of the BIPM and in the preparation of its work program. It is necessary to also mention the laboratories of the Euratom Central Bureau of Nuclear Measurements and of the International Atomic Energy Agency, with which the BIPM maint ains close relations.

\section{Units and the International System of Units}

The authority of the CGPM is recognized worldwide with respect to all matters concerning units and unit systems. Its decisions are taken over into the legislation of numerous countries, even if they do not yet adhere to the Metre Convention.

In 1913, France had presented its draft of metric legislation to the 5 th CGPM. The question of an international systen of measurement units was not, however, truly submitted to the CGPM until 1948: the International Union of Physics requested the establishment of an international practical system of units; for its part, the French delegation proposed a draft of a universal system of measures as a basis for discussion. The 9th CGPM charged the CIPM "to open an official inquiry on the opinion in scientific, technical, and pedagogical circles of all countries, to centralize the responses, and to issue recommendations respecting the establishment of a single system of practical measurement units, suitable for adoption in all the signatory countries of the Metre Convention." 
The results of this inquiry led finally to the adoption by the 10th and 11th CGPM (1954 and 1960) of the International System of Units, designated by the symbol SI in all languages. This coherent system, currently based on seven base units (app. 4, p. 238) and two supplementary units, has been adopted by the principal international organizations; it is progressively replacing, in national legislation, the various systems (CCS, MTS, MKS, etc.) growing out of the metric system and the Anglo-Saxon measures in the countries where metric conversion is under way.

For the study of questions about units, the CIPM had instituted a "Commission on a System of Units" in 1954; in October 1964, this became the Consultative Committee for Units (CCU). This Consultative Committee collaborates closely with the other international organizations ${ }^{11}$ which, in their respective fields, are led to concern themselves with units of physical quantities, and with their nomenclature and symbols.

The CCU held its first meeting in April 1967. During its later meetings it prepared a document on the SI, supplemented with information on the practical realization of the definitions of the principal units [4].

At the moment, there exists no Consultative Committee for mass measurements, one of the basic activities of the BIPM, nor for gravimetry (absolute determination of the acceleration of gravity), another important activity of the BIPM. For the unit of mass, no natural standard has yet been proposed which can advantageously replace the International Kilogram, which has always accurately rendered the service expected of it; the CIPM has thus never had a motive for setting up a committee of experts on this subject. As to gravimetric measurements, discussions on this subject have taken place in the specialized committees of the International Union of Geodesy and Geophysics.

The creation of various Consultative Committees marks the evolution of the activities of the BIPM. It should not hide the permanent tasks which fall on the BIPM and which constitute the major part of its work:

- international comparisons, most often organized by the competent Consultative Committees, which permit verification of the consistency of the basic determinations carried out in different laboratories:

- verification of standards of numerous countries, verifications which since 1883 have given rise to the issuance of about 4000 certificates, study notes, and reports;

\footnotetext{
${ }^{11}$ International Electrotechnical Commission (IEC), International Commission on Illumination (CIE), International Commission on Radiation Units and Measurements (ICRU). International Organization for Standardization (ISO). International Union of Pure and Applied Physics (IUPAP), International Union of Pure and Applied Chemistry (IUPAC).
} 
- the assistance that the BIPM supplies, within its means, to national or international institutions in the field of source standards for metrology. ${ }^{22}$

Thus the BIPM has never ceased working effectively to assure the uniformity of measurements in the entire world, thanks to the propagation of the Metric System and then of its direct descendant, the International System of Units (app. 5, p. 243 ).

${ }^{12}$ In the past, the BIPM had also been induced to lend its assistance in the preparation of national laws on weights and measures; in 1933, it was proposed to the 8th CGPM to extend the scope of the organs of the Metre Convention to legal and commercial metrology, and to create a "Consultative Committee for Applied Metrology" under the CIPM. The independence of such an organism with regard to the CIPM and the BIPM having been judged preferable, this proposal finally resulted in the 1955 creation of the International Organization for Legal Metrology and of the International Bureau of Legal Metrology located in Paris.

\section{Bibliography}

[1] Documents diplomatiques de la Conférence du Mètre, Paris, Imprimerie Nationale, 151 pages (1875).

[2] La création du Bureau International des Poids et Mesures et son oeuvre. Gauthier-Villars. Paris, 321 pages (1927). See also, for example:

Bigourdan, G., Le Système métrique des poids et mesures: son établissement et sa propagation graduelle, avec l'histoire des opérations qui ont servi à déterminer le mètre et le kilogramme. Gauthier-Villars, Paris, 458 pages (1901).

Favre, A., Les origines du Système métrique. Presses Universitaires de France, Paris. 242 pages (1931).

Moreau, H., Le Système métrique: des anciennes mesures au Système International d'Unités. Chiron, Paris, in press.

[3] Benoît, J.-R., Rapport sur la construction, les comparaisons et les autres opérations ayant servi à déterminer les équations des nouveaux prototypes métriques, Travaux et Mémoires B.I.P.M. 7, 132 pages (1890).

[4] Le Système International d'Unités (SI), 2 d edition B.I.P.M., 40 pages (1973). The Inter: national System of Units (SI), 1974 edition, NBS Special Publication 330, 43 pages. and The International System of Units (SI), HMSO, London (1973). 



\section{CHAPTER II}

\section{MASS}

In its Article 6, the Metre Convention charged the International $\mathrm{Bu}$ reau of $W$ eights and Measures with conserving the international prototypes of the metre and kilogram, and with comparing them with national standards.

The BIPM was thus the unique possessor of the starting-point standards for all measurements of length and mass; it devoted itself entirely to these fields of activity and, naturally, to indispensable measurements of certain auxiliary quantities: temperature, pressure, density ...

Later, the activities of the BIPM were extended to several other fields, for example to measurements of electric quantities, measurements in photometry and in the field of ionizing radiations, but none of these measurements was based on a new material primary standard; moreover, in 1960, the change of the definition of the metre took away from the international prototype metre its role as primary standard for length measurements, so that the international prototype kilogram remains the only primary standard conserved by the BIPM.

Today, all sufficiently well equipped laboratories can, in principle, realize the units of length, time, temperature, and luminous intensity, which are defined in terms of natural properties of certain atoms or certain substances; on the other hand, these laboratories need the BIPM for the availability of the unit of mass and for realization of the units of electric current and amount of substance, whose definitions bear directly or indirectly on a mass. It is the same for such derived units as those of force, pressure, and energy, which depend on the base units and particularly on the unit of mass.

Conservation of the international prototype kilogram and verification of national mass standards thus continue to be a fundamental part of the mission assigned to the BIPM by the Metre Convention.

\section{The Prototype Kilograms and Periodic Verifications}

\section{The International Kilogram}

In 1878, three platinum-iridium cylinders (later identified by the symbols KI, KII, and KIII) were ordered from Johnson, Matthey and Co. of 
London. They were polished and adjusted by A. Collot, in Paris, and compared with the Kilogram of the Archives by four obscrvers in 1880 at the Obscrvatory of Paris (the BIPM did not yet have available the scientific equipment needed for this work).

Cylinder KIII, whose mass was found to be the closest to that of the Kilogram of the Archives, was sent to the BIPM, chosen as the international prototype kilogram in 1883, and ratified as such by the 1st CGPM (1889). The definition of the unit of mass resulting from this was explicitly stated by the 3d CGPM (1901) in the following terms: "The kilogram is the unit of mass; it is equal to the mass of the international prototype of the kilogram." This prototype, called "International Kilogram," is frequently designated by the symbol 迹.

The alloy of platinum (90\%) and iridium (10\%) had been chosen to make 政 as invariable as possible. On the one hand, this alloy is not subject to deterioration: on the other hand, the iridium gives it a hardness which reduces the wear on the prototype during the various manipulations to which it is subjected. The good quality of the polish which can be obtained also contributes to this invariability: it reduces the effect of high spots exposed to wear, and that of low spots which could retain dust. The density of the alloy (around $21.55 \mathrm{~kg} / \mathrm{dm}^{3}$ ) is close to that of the platinum in the Kilogram of the Archives. Thus the volumes of these two standards being closely the same, the difference of the buoyancies produced by the air during their comparison was small, and the corresponding correction could be determined without too much difficulty. The surface of a mass standard should be as small as possible to further reduce the causes of variation either by wear or by adsorption of various substances. From this point of view, the most advantageous form is the sphere, but taking account of the difficulties of manufacture, adjustment, and especially handling, the minimum-area cylindrical form is preferred, that is to say, a cylinder whose height is equal to its diameter ${ }^{1}$ with, however, its edges rounded.

Such a standard must naturally be handled with extreme care; we use specially adapted tweezers furnished with velvet or chamois skin, and kept sheltered from dust. This does not, however, dispense with using a small brush to wipe those regions which will come into contact with the standard, before using the tweezers. It is absolutely necessary to avoid dragging the standard on its support; this would inevitably cause a loss of mass. To make 迹 lose a mass of $0.01 \mathrm{mg}$ by uniformly wearing one of its bases, it would suffice to remove a thickness of metal of only $0.0004 \mu \mathrm{m}$, that is to say, to remove a little more than a layer of atoms. The surest means of assuring the invariability of the unit of mass is therefore to make the least possible use of $\bar{i}$. This is why it is removed only very rarely from the vault

\footnotetext{
${ }^{1}$ The corresponding surfacc area is only 1.310 times that of a sphere of the same volume. The height of the International Kilogram. equal to its diameter. is about $39 \mathrm{~mm}$; the area of each of its bases is about $12 \mathrm{~cm}^{2}$ and its total surface area is $72 \mathrm{~cm}^{2}$; its volume is $46.4018 \mathrm{~cm}^{3}$.
} 
for the metric prototypes where it is kept, under three glass domes, well sheltered from pollution (p. 30).

\section{Other Prototype Kilograms}

In 1882, 40 platinum-iridium cylinders were ordered from Johnson, Matthey and Co. They were delivered in 1884 and their density was determined; they were then polished and adjusted (to about $\pm 1 \mathrm{mg}$ ) by A. Collot at the BIPM. The numbers 1 to 40 were assigned to them. ${ }^{2}$ Counting the standards KI and KII which remained available after the choice of the International Kilogram, 42 Prototype Kilograms ${ }^{3}$ were thus available, in all respects similar to 15 . These standards, after cleaning with steam and alcohol vapor, were compared among themselves in a very large number of combinations of pairs, and each was compared directly with $\mathrm{pz}$.

During the 1st CGPM, 34 standards were assigned by lot to those Member States of the Metre Convention which had requested them. Prototype Nos. 9 and 31 were assigned to the BIPM as working prototypes; KI and No. 1 became the two check standards for and were deposited in the same vault; Nos. 7, 8, 29, and 32 remained available.

Subsequently, to be able to respond to requests, 23 other prototype kilograms were constructed from 1929 to 1974, starting with cylinders prepared either by Johnson, Matthey and Co. or by Comptoir Lyon-Alemand at Paris, some of them recast from old prototypes. Nine of them were adjusted at the BIPM. These 23 prototypes carry the numbers 41 to 63 . Only the last remains available, the 22 others having been assigned, along with No. 29.

In 1905, prototypes No. 8 and No. 32 became two new check standards for 焉. In 1925, No. 7 replaced check standard No. 1 which had been subject to an accident during a weighing. ${ }^{4}$ In 1938 , prototypes 43 and 47 were used to increase the number of check standards for $\bar{k}$, which brought this number to six.

Finally, No. 25, assigned to France and which had been given to the Paris Observatory, was ceded to the BIPM in 1958 and has become a prototype for infrequent use.

\footnotetext{
2 These numbers are marked on the sides of the cylinders by a slight depolishing through a stencil.

${ }^{3}$ Mass standards of 1-kg nominal value are called "Kilograms." The designation "Prototype Kilogram" (or simply "prototype") is generally reserved for platinum-iridium Kilograms.

${ }^{4}$ It was then noticed that prototype No. 1 had a stightly convex base; this construction defect was the cause of its fall.
} 


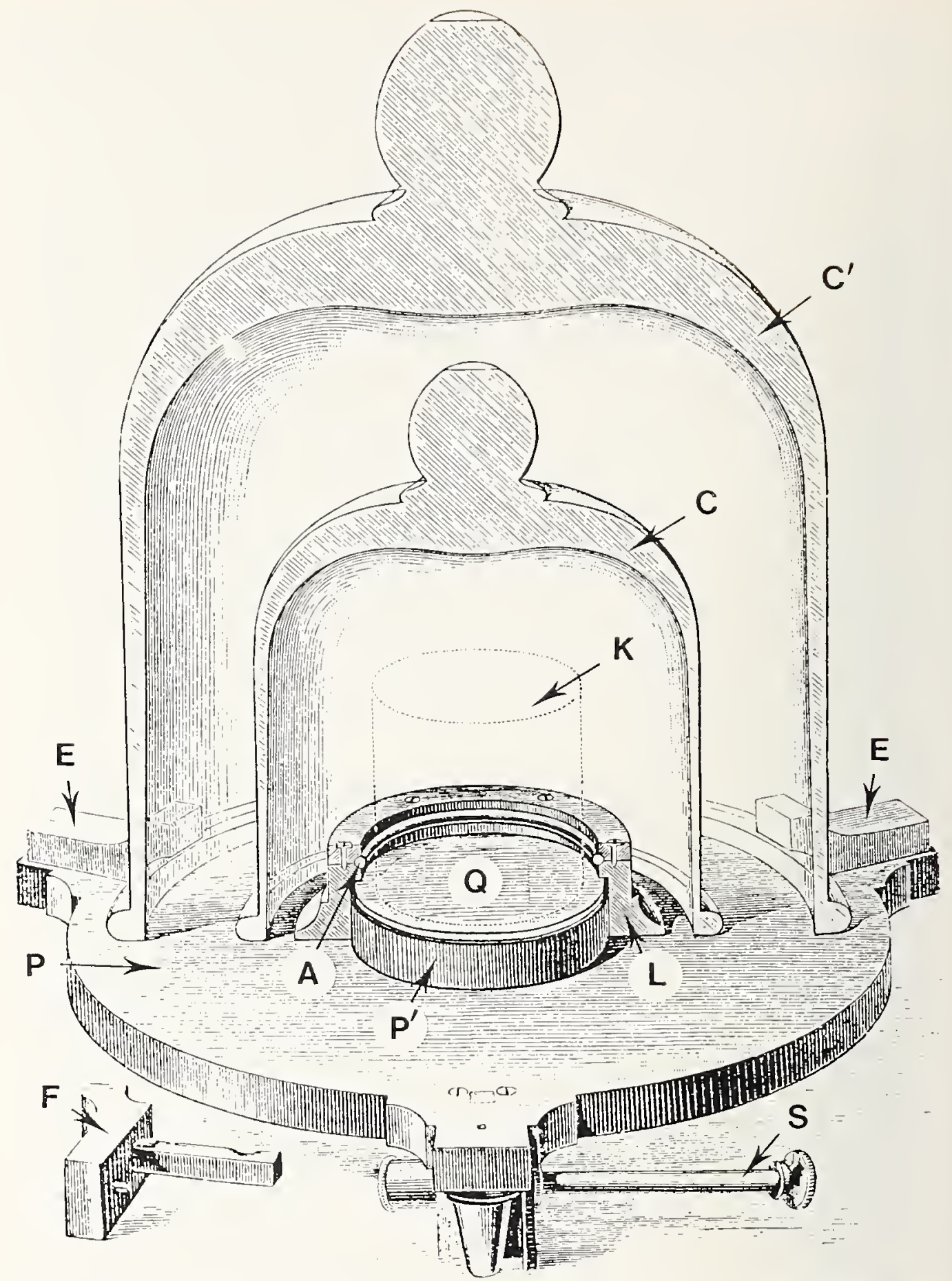

Figure 1. Housing used for the protection of prototype Kilograms and for transporting them inside the laboratory. The prototype Kilogran $\mathrm{K}$ rests on a quartz plate $Q$, which in turn rests on a brass cylinder $P^{\prime \prime}$ attached to the plate $P$. Its possible lateral displacements are limited by a brass erown $L$ provided with a silver ring $A$ whose inside diameter is $3 \mathrm{~mm}$ greater than the diameter $(39 \mathrm{~mm})$ of the Kilıgram. The latter is covered by two glass domes $\mathrm{C}$ and $\mathrm{C}^{\prime}$, one of which is held in place by blocks resting on the rim at the base of the dome. The two blocks E are fixed: block F. detachable. can be immobilized by the key $S$ to assure clisure of the housing.

The housing for the International Kilogram $\mathbb{R}$ is itself protected by a third dome resting on a ground-glass plate. 
Article 6 of the Metre Convention provided for periodic comparisons of national prototypes with the international prototype or its check standards; it is the duty of the BIPM to organize these.

The first periodic verification of the Prototype Kilograms was carried out between 1899 and 1911. It involved 18 national prototypes, 4 prototypes not yet assigned, the 2 working prototypes of the BIPM, 2 other BIPM prototypes designated $\mathrm{C}$ and $\mathrm{S}$ whose forms were respectively a cylinder and a truncated sphere, and finally prototype No. 1, check standard for

This important comparison was carried out without preliminary cleaning of the prototypes. The results, communicated to the 5th CGPM in 1913 [1], made it evident that the masses of most of the prototypes had practically not changed relative to their values in 1889 . In particular, the masses of those which had not been used were often found constant to a few micrograms. This was the case for No. 1, check standard for 1 , for Nos. 8 and 32, new check standards since 1905, and also for No. 21, assigned to Mexico and which had made the round trip without being unpacked. On the other hand, the masses of certain prototypes which carried visible signs of wear or accident had diminished appreciably. One of the BIPM working prototypes (No. 31), which had been handled with great care but had been subjected to particularly intensive use, had itself lost $21 \mu \mathrm{g}$. The possibility of regularly comparing the working prototypes to one or two check standards and the recent increase in the number of check standards indicated that conservation of the unit of mass was assured to better than $0.01 \mathrm{mg}$ for many years, even having recourse to 3 only very rarely.

In light of this reassuring conclusion, the CIPM waited nearly 30 years before requesting the BIPM to organize the second periodic verification of National Prototype Kilograms, preceded this time by a comparison of with its check standards. This comparison was carried out in June 1939 [2]; it involved the four old check standards (KI, Nos. 7, 8, and 32) and the two new ones, Nos. 43 and 47 , to which it was necessary to assign values. This comparison was itself preceded by comparisons among check standard No. 8, several prototypes not yet assigned, and the BIPM prototypes C, S, No. 9, and No. 31. All these kilograms were cleaned before weighing with a chamois skin impregnated with alcohol and then with redistilled benzene. The results obtained were not very satisfying: they seemed to show for the four old check standards and the working prototype No. 9 an increase in mass (of 0.03 to $0.08 \mathrm{mg}$ ) relative to their values in 1889; working prototype No. 31, whose bases carried numerous lines and traces of rubbing seemed to have conserved its original value. The following explanation was put forth: although is kept under three glass domes, of which the largest, furnished with a stopcock by which a partial vacuum was made, rested on a ground-glass plate, the other prototypes are only placed on their support under two domes which rest on a metal plate (fig. 1). 
Changes of the air around them have probably been more important than around and it has been possible for the formation on these check standards of adherent deposits which a washing in steam or alcohol vapor, not done in 1939, would doubtless remove.

The following years were put to profit in studying the cleaning of prototypes. The cfficacy of washing with steam was recognized and it was decided to apply this treatment, before each important group of com. parisons, to prototypes previously cleaned with pure benzene and pure alcohol. Before each weighing, a fine hair brush was also used on the entire surface of the prototype to remove most of the dust.

In 1946, a new comparison was made among 1 , its six check standards (KI, Nos. 7, 8, 32, 43, 47), and the working prototypes Nos. 9 and 31. For the four old check standards, the small changes $(-0.03$ to $+0.04 \mathrm{mg})$ found relative to the 1889 values showed the importance of cleaning and washing the prototypes as regards the confidence which can be placed in the weighing results [3]. This comparison was followed, between 1948 and 1953, by the second periodic verification of the national prototype kilograms. Thirty-three prototypes participated therein. The results, presented to the 10th CGPM in 1954 [4], demonstrated the perfect stability of certain prototypes; in many cases, the verified variation of others could be explained.

No other verification of national prototype kilograms has been organized since.

Thus, as is desirable, ${ }^{2}$ has been used only in rare circumstances: comparisons before 1889 (when it was involved in some 40 weighings), comparisons of 1939 ( 7 weighings), and of 1946 (14 weighings).

\section{Determination of the Mass of Kilograms Outside the Periodic Verifications}

The BIPM can naturally study each kilogram for which a request is made. Thus since 1953, date of the end of the second periodic verification, it has determined the density and the mass of 7 newly constructed prototypes and it has verified 16 other prototypes which are, for the most part, national prototype kilograms.

For these comparisons, the BIPM has available its two working prototypes, Nos. 9 and 31. Since these are subject to intensive use (each is manipulated from 300 to 500 times per year) and their surface is consequently exposed to certain pollution and to possible wear, it is necessary to redetermine their mass from time to time. To avoid the need of removing one or two check standards for from the vault, an operation which requires CIPM authorization, the CIPM decided in 1956 to place at the disposal of the BIPM a prototype for infrequent use, No. 25, which should 
be used only rarely. It is kept in a vault to which the Director of the BIPM has easy access.

The masses of prototypes Nos. 9, 31, and 25 had been determined at the time of the second periodic verification, then in 1957 by comparison with the six check standards for 19 and in 1965 by comparison with two check standards Nos. 8 and 43. Since that date, the masses of Nos. 9 and 31 have been redetermined by comparison with No. 25, on two occasions, in 1968 and in 1973. A redetermination is anticipated about every 5 years. The study of the history of the mass of these two prototypes is very instructive (fig. 2). It shows that the loss of mass by wear can be extremely small for prototypes that are manipulated with great care, but that on the other hand their exposure to the laboratory air produces a pollution of their surface which betrays itself by a gain in mass of the order of $3 \mu \mathrm{g}$ per year. Cleaning with a solvent followed by washing in steam from twice distilled water makes them recover their initial mass very closely.

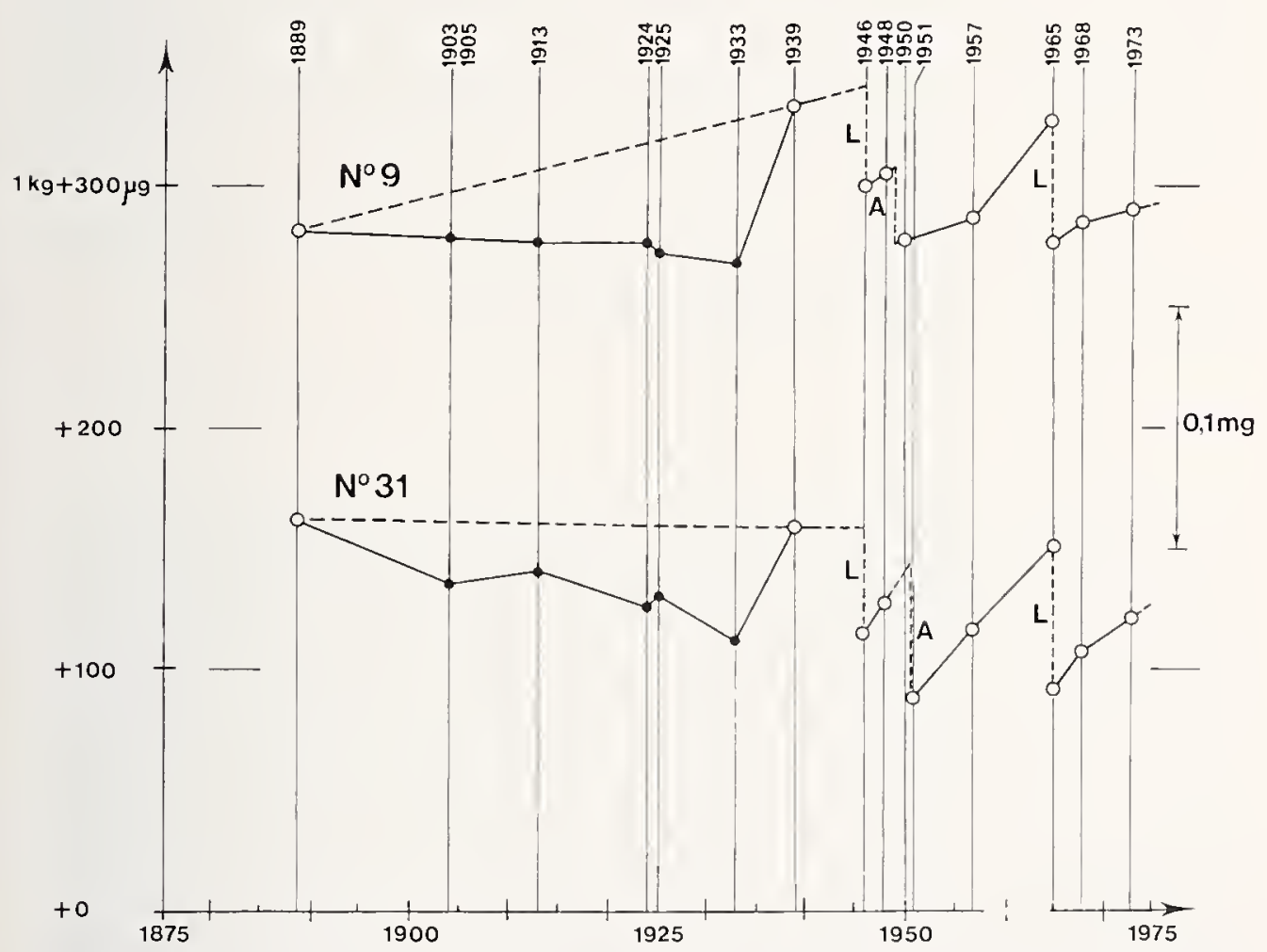

Figure 2. History of the mass of the BIPMI working prototypes Nos. 9 and 31 . For the two prototypes, we clearly see the effect of steam washing $L$ practiced in 1946 and 1965, as welt as the increase of their mass between washings and since 1965. This increase is less for No. 9 than for No. 31 whose surface is rather strongly grooved. The values obtained from 1903 to 1933 result from a smaller number of weighings and are traccable to $7 \mathbf{z}$ only in a very in direct way: they are less reliable than those of 1889 and 1939. Between these two dates, the wear on No. 31. used very intensively, was compensated by the mass gain due to pollution of its surface. The discontinuities A correspond to accidents underone by the two prototypes in September 1949 and February 1951, respectively. 
Thanks to rescarch pursued since the beginning of the century on the fabrication of mass standards in a material less costly than platinum-iridium, there are now available alloys whose stability is quite satisfactory. In particular, stainless steel containing about 20 percent nickel and 20 percent chromium has been used for the construction of some 15 national kilograms which have been studied by the BIPM. The density of this alloy being around $7.8 \mathrm{~kg} / \mathrm{dm}^{3}$, the volume ${ }^{5}$ of these kilograms is about $82 \mathrm{~cm}^{3}$ greater than that of the platinum-iridium prototypes. As a result, when the mass of a stainless steel kilogram is determined by comparison with a platinum-iridium prototype, the different values of air buoyancy on the two standards requires the application of a correction whose value reaches nearly $100 \mathrm{mg}$ at the BIPM. This correction can not, at this time, be calculated to better than $0.05 \mathrm{mg}$, by reason of the limited precision with which the density of air is known as a function of ambient conditions (pressure, temperature, humidity).

But it must be noted that if a laboratory, starting with a stainless steel standard kilogram, calibrates secondary standards of the same material, no uncertainty due to air buoyancy affects this measurement; on the other lıand, if a platinum-iridium prototype kilogram is used, the uncertainty in the correction for air buoyancy at that moment enters into the determination.

\section{Balances and Methods Used for Comparisons of Kilograms; Auxiliary Measurements}

\section{Balances and Weights}

The only method allowing comparison of two masses with a precision better than $1 \times 10^{-8}$, that is, with an uncertainty less than $0.01 \mathrm{mg}$ for $1-\mathrm{kg}$ masses, consists of comparing their weights by means of a balance. Since its beginning, the BIPM has been equipped with several "equal-arm" balances. It is easily realized that it is not possible to adjust the lengths of the arms of a balance beam with the desired precision, that is, to better than $2 \mathrm{~nm}$ for arms of $0.2 \mathrm{~m}$. One is thus led to use the balances in a doubleweighing operation, either by Borda's method which consists of substituting one mass for the other on the same platform, or by Gauss's method in which the masses on the two platforms are interchanged. The Bunge balance and the Rueprecht balance, which until recently were the two principal balances used at the BIPM for Kilogram comparisons [5], were designed for operation with Gauss's double weighing. The observer manipulates the balance from a distance of $4 \mathrm{~m}$, the balance case remain-

\footnotetext{
${ }^{5}$ The stainless steel Kilograms have a volume of about $128 \mathrm{~cm}^{3}$. They have the form of a cylinder with rounded edges, whose height, equal to the diameter, is about $55 \mathrm{~mm}$.
} 
ing closed; in particular, he controls the movement of the transposition mechanism which accomplishes the permutation of the masses. Temperature variations which would be caused by the approach of the observer are thus avoided.

The masses of the Kilograms to be compared are generally very close; if such is not the case, additional loads of known value are added to the standard of lesser mass, in preparing for the weighing. For each of the two placements of the masses $m_{1}$ and $m_{2}$ on the platforms, the beam inclination corresponding to its equilibrium position is determined. If the lengths of the two arms of the beam are designated by $l_{1}$ and $l_{2}$, these very small inclinations are proportional to $m_{1} l_{1}-m_{2} l_{2}$ for the first arrangement and $m_{2} l_{1}-m_{1} l_{2}$ for the second. Their difference $\alpha$ is proportional to

$$
\left(m_{1} l_{1}-m_{2} l_{2}\right)-\left(m_{2} l_{1}-m_{1} l_{2}\right)=\left(m_{1}-m_{2}\right)\left(l_{1}+l_{2}\right)
$$

The coefficient of proportionality is related to the sensitivity of the balance which is slightly variable in high-precision balances by virtue of the close proxinity of the beam axis (edge of the central knife) and its center of gravity. This coefficient is determined for each weighing by means of small masses $m^{\prime}{ }_{1}$ and $m^{\prime}{ }_{2}$ (for example, $100.5 \mathrm{mg}$ and $100.0 \mathrm{mg}$ ) whose difference is well known and which are placed, by means of a remote control mechanism, on parts called stirrups which form part of the platform suspension; the platforms remain loaded by the Kilograms under comparison. The additional inclination $\beta$ which results is proportional to

$$
m_{1}^{\prime} l_{1}-m_{2}^{\prime} l_{2} \approx\left(m_{1}^{\prime}-m_{2}^{\prime}\right)\left(l_{1}+l_{2}\right) / 2
$$

from this is easily derived

$$
m_{1}-m_{2}=\frac{\alpha}{2 \beta}\left(m^{\prime}{ }_{1}-m^{\prime}{ }_{2}\right)
$$

The availability, in the balance case, of two identical sets of additional loads allows loading the beam on eitler the left or the right.

In practice, a graduated scale is observed through a telescope by reflection in a plane mirror attached to the balance beam. When the latter oscillates, the graduated scale appears to march past a fixed reference point. The maximum excursions successively attained to the riglit and left are noted. Starting with three successive excursions $x_{1}, x_{2}, x_{3}$, the calculation of the excursion $x$ corresponding to the equilibrium position of the beam can be made: $x=\left(x_{1}+2 x_{2}+x_{3}\right) / 4$. Five successive excursions arc generally noted, allowing judgment of the regularity of the oscillation. Similarly, one is not content with a single transposition of the masses but usually uses 10 of them; this allows determining 6 equilibrium inclinations for one disposition of the masses and 5 for the other. The agreement of successive equilibrium positions indicates the quality of the weighing and the good condition of the balance.

To avoid having the transposition mechanism come into direct contact with the Kilogram base during a weighing, the kilograms in fact sit on 
platinum auxiliary platforms. The masses of the auxiliary platforms are adjusted as well as possible; their difference, which can reach $0.02 \mathrm{mg}$, is eliminated from the results of the comparison by a complete weighing comprising two parts between which the Kilograms are interchanged without interchange of the auxiliary platforms.

The Bunge balance, in service since 1879 , has shown itself difficult to regulate in a lasting way. After the fall of the two BIPM working prototypes (No. 9, in 1949, during the preparation for a weighing, and No. 31, in 1951, in the course of a weighing) in this balance, we have stopped using it.

The Rueprecht balance, delivered to the BIPM in 1878, was completely overhauled and modified by its maker in Vienna in 1900-1902 [7]. In 1937, after prolonged and intensive use, it was sent to C. Longue, in Paris, who succeeded in restoring it to practically its original quality. Since then, we have applied ourselves mostly to improving its installation, notably its thermal protection and the precision of measurement of temperat ure inside the balance case. Despite the care with which this balance has been used, it has probably lost part of the exceptional quality that it previously had. In fact, from its last reconditioning until the end of 1974, it has been used for about 1000 complete weighings, representing about 250000 oscillations of the beam. The releasing of the balance, which places the knives in contact with their flats, has thus been repeated about 30000 times. The frequency of use now tends to diminish since the BIPM has had available since 1970 , a new balance placed into service in 1973, based on an entirely different concept and having higher precision (see p. 56).

\section{Calibration of Multiples and Sub-Multiples of the Kilogram}

The accuracy of a weighing depends primarily on the accuracy with which are known the smallest masses, notably those which are used as additional loads in Kilogram comparisons. Calibration of a series of masses allowing passage from the Kilogram to the milligram is thus a fundamental operation.

Consider a series comprising the masses $m_{500}, m_{200}, m^{\prime}{ }_{200}$, and $m_{100}$ of nominal values $500 \mathrm{~g}, 200 \mathrm{~g}, 200 \mathrm{~g}$, and $100 \mathrm{~g}$, and also masses $m_{50}, m_{20}$, $m^{\prime}{ }_{20}$, and $m_{10}$ whose sum of values, designated by $\Sigma_{100}$, is nominally equal to $100 \mathrm{~g}$. The methods used for calibrating such a series always come back to a determination of the small difference $\epsilon$ which exists between combinations of different masses giving the same nominal value. Thus, commencing by comparing a Kilogram with the set of masses of nominal values 100 g to $500 \mathrm{~g}$, we obtain:

$$
m_{500}+m_{200}+m_{200}^{\prime}+m_{100}=1 \mathrm{~kg}+\epsilon_{1}
$$


and then, for example, the following comparisons are made:

$$
\begin{aligned}
& m_{500}-\left(m_{200}+m_{200}^{\prime}+m_{100}\right)=\epsilon_{2} \\
& m_{500}-\left(m_{200}+m_{200}^{\prime}+\Sigma_{100}\right)=\epsilon_{3} \\
& m_{200}+m_{100}+\left(m_{200}^{\prime}+\Sigma_{100}=\epsilon_{4}\right. \\
& m_{200}+\Sigma_{100}-\left(m_{200}^{\prime}+m_{100}\right)=\epsilon_{5} \\
& m_{200}-m_{200}^{\prime}=\epsilon_{6} \\
& m_{200}-\left(m_{100}+\Sigma_{100}\right)=\epsilon_{7} \\
& m_{200}^{\prime}-\left(m_{100}+\Sigma_{100}\right)=\epsilon_{8} \\
& m_{100}
\end{aligned}
$$

This linear system of nine equations in five unknowns $\left(m_{500}, m_{200}, m^{\prime}{ }_{200}\right.$, $\left.m_{100}, \Sigma_{100}\right)$ is reduced by the method of least squares to a linear system of five equations in five unknowns which can be solved by hand, but for which a computer is now preferred.

The next step follows analogously since $\Sigma_{100}=m_{50}+m_{20}+m_{20}^{\prime}+m_{10}$ is now known. For the final step, the series must be complemented by a supplementary $1 \cdot \mathrm{mg}$ mass. The final result is the calibration of masses of the order of a milligram with an uncertainty of about $0.5 \mu \mathrm{g}$.

Naturally, the same method is applicable in going from $1 \mathrm{~kg}$ to $10 \mathrm{~kg}$. The BIPM has available balances adapted to various loads which occur in these different weighings.

\section{Hydrostatic Weighings and Correction for Air Buoyancy}

Accurate weighings require the application of a correction for "air buoyancy." Its calculation involves the volumes of the standards being compared, volumes which are generally determined by hydrostatic weighings.

A hydrostatic weighing consists essentially in determining the mass of liquid displaced by a submerged body. If the volume of the body is known, the density of the liquid can be deduced; conversely, if the liquid density is known, the volume of the body is obtained. The volume of a Kilogram is thus determined by using water as the immersion liquid.

A hydrostatic weighing is carried out by the Borda double weighing procedure. In a first operation, the submerged body is suspended from the balance and equilibrium established; in the second operation, the body is freed from the suspension system by means of a mechanism inside the immersion vessel, and equilibrium is reestablished by substituting masses on the platform of the same side. This yields equality between the apparent mass $m-m_{\mathrm{w}}$ of the submerged body (its real mass reduced by the mass of the displaced liquid) and that of the mass added to the platform (real mass reduced by the mass of the displaced air).

By weighing the same body in air, its apparent mass $m-m_{\mathrm{a}}$ (real mass reduced by the mass of displaced air) is, in fact, determined.

Introducing the volume $V$ of the body under study, and the densities 
$\rho_{\mathrm{w}}$ and $\rho_{\mathrm{a}}$ of the liquid and of air, it is seen that one has determined the two quantitics $m-V \rho_{\mathrm{a}}$ and $m-V \rho_{\mathrm{w}}$ whosc differcnce is $V\left(\rho_{\mathrm{w}}-\rho_{\mathrm{a}}\right)$.

For water, wc calculate $\rho_{w}$ for the conditions of measurement starting with the value $999.972 \mathrm{~kg} / \mathrm{m}^{3}[6,7,8]$ assumed for standard reference conditions $\left(4^{\circ} \mathrm{C}\right.$ (maximum density), standard atmospheric pressure, absence of dissolved gas), and using the expansion table of Chappuis [9]. For air, we calculate $\rho_{\mathrm{a}}$ starting from ambient conditions: temperature and relative humidity in the balance case, and atmospheric pressure, by means of formulas due to Broch [10].

One must allow for the fact that the acceleration of gravity does not have exactly the same value at the level of the submerged body and at the level of the masses on the platform.

The volumes of Kilograms are thus determined to about $1 \times 10^{-5}$. They enter into the comparisons through calculation of the correction due to air buoyancy.

The hydrostatic balance which was delivered to the BIPM in 1891 to replace the balance then in service has itself been replaced in 1963 by a new balance of more recent concept.

\section{Recent Balances and Current Studies}

\section{New Hydrostatic Balance; Density of Water}

This balance (fig. 3), whose assembly was accomplished in 1964, is furnished with a remotely operable mechanical device for substitution of a mass on one level of the platform, for a mass on the other level of the same platform.

The small additional loads used in determining the balance sensitivity, that is, actually, for calibrating the divisions of the reading scale "in mass," can be placed on the stirrups or removed without the observer coming near the balance. We have concentrated on checking and improving the uniformity of the water temperature in the immersion vessel. With this new instrument, the density of Kilograms can be determined with a reproducibility better than $1 \times 10^{-6}$. Figure 4 gives the principle of such a measurement.

This balance was reconditioned in 1973-1974. On that occasion, it was equipped with a brake which permitted limiting the amplitude of oscillation to a determined value, and with a mechanism for changing masses on the two levels of the right-hand platform. identical to that which already existed for the left platform. It will be possible to carry out differential hydrostatic weighings between two baths (for example, water at different temperatures or of different isotopic constitution) with the help of two submerged bodies of approximately the same mass and volume. 


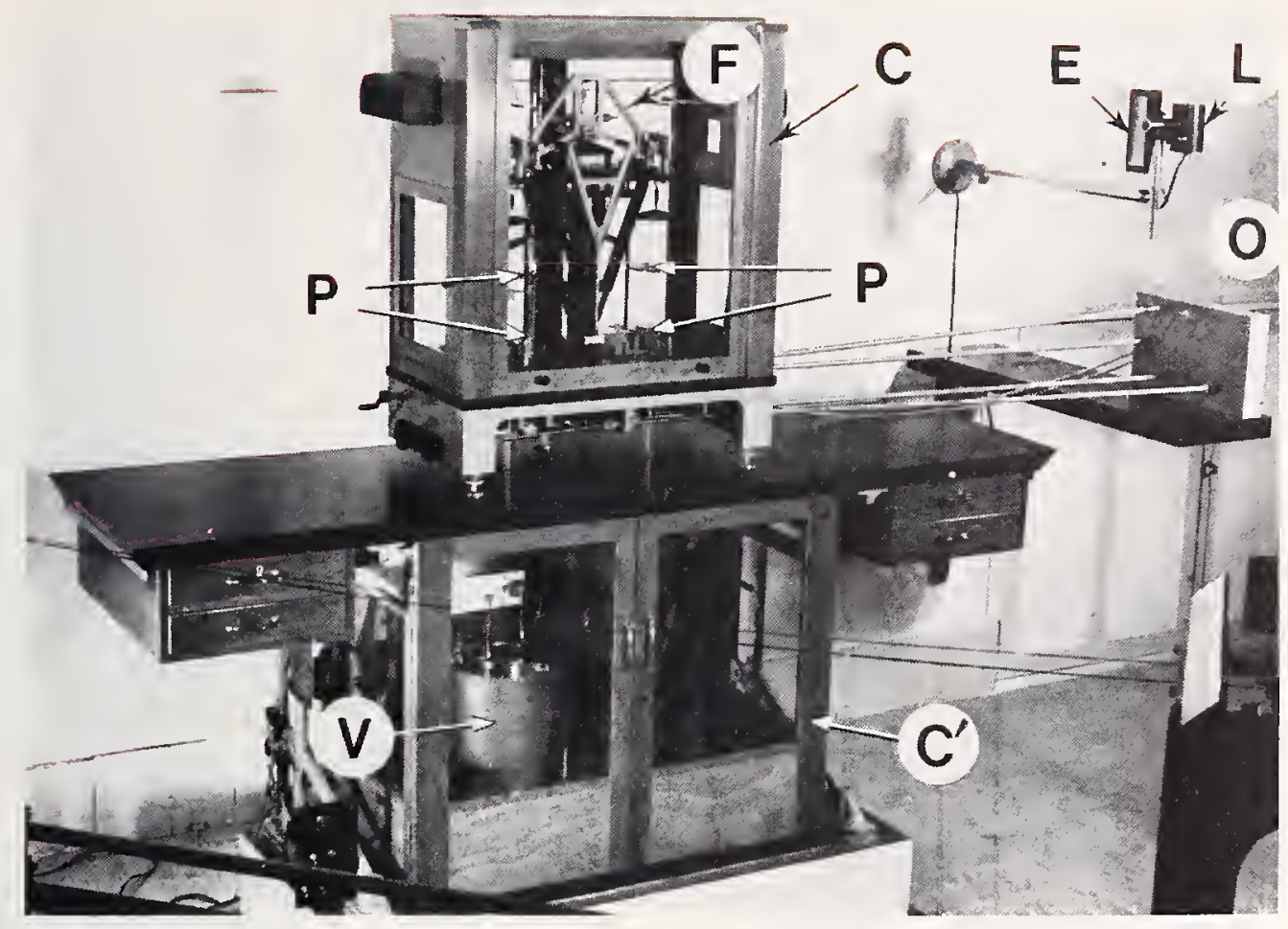

Figure 3. The hydrostatic balance installed in 1963. C. upper case containing the balance; C'. lower casc containing the immersion vessel V: F, beam: P, two-level platform; E, ubservation scale: L. projector: O, position of the observer operating the balance controls, from a distance of $2 \mathrm{~m}$. The thermal shields have been removed.

The precision of this balance has allowed us to undertake, starting in 1967, the study of the variation of density of water as a function of its isotopic constitution [11], that is to say, of its content of heavy isotopes ${ }^{18} \mathrm{O},{ }^{17} \mathrm{O}$, and ${ }^{2} \mathrm{H}$. The body used for the hydrostatic weighings is a stainless steel cylinder of approximately $1 \mathrm{~kg}$ mass, whose volume and expansion coefficient have previously been determined. Observations have been made on seven samples of water which have been distilled twice before the weighings: two samples from Greenland (one in the solid state at an altitude of $2900 \mathrm{~m}$, the other from a small lake near Thule), three samples of sea water (two from the Atlantic and one from the Mediterranean), and two samples of water artificially enriched in ${ }^{18} \mathrm{O}$. At the end of each weighing, a sample was taken for analysis at the Saclay Center for Nuclear Studies. The values obtained cover a range of $0.02 \mathrm{~kg} / \mathrm{m}^{3}$, which represents the maximum spread due to only the difference of isotopic composition which can be encountered in practice between the densities of any two samples of natural water. A formula established from these results allows calculation of the difference of densities between any two natural waters of known isotopic constitution to about $0.001 \mathrm{~kg} / \mathrm{m}^{3}$.

This study poses anew the problem of the density of water: it demonstrates the need for determining in an absolute way, at a reference tem- 
perature, the density of water of known isotopic constitution and for making new measurements of the variation of water density as a function of temperature. Several laboratories have already started these studies at the request of international organizations cooperating with the BIPM.

\section{Single-Platform Balance for Comparing Kilograms}

For some decades, several national laboratories have studied balances having an essential improvement which consists in realizing the interchange or substitution of the masses being compared while maintaining cont act between the knives and the flats. This largely avoids disturbing the molecular relations which become established when the knives are in contact with the flats; there results a better constancy of the length of the beam arms and consequently a better accuracy of the balance.
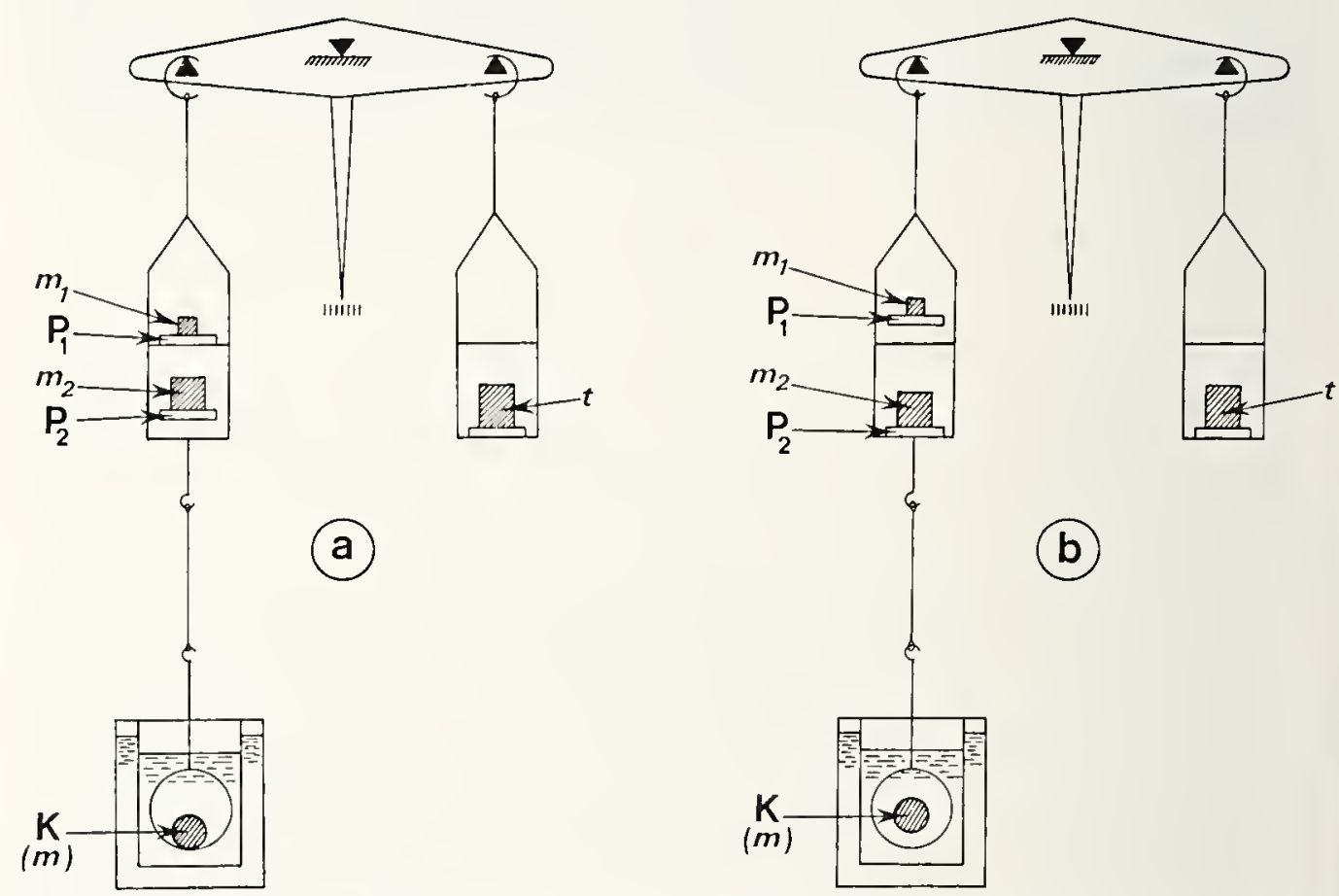

Figure 4. Scheme of utilization of the hydrostatic balance. a) First opcration. The submerged hody $\mathrm{K}$ (Kilogram) of mass $m$ is suspended from one of the platforms. The halance is equilibrated with the mass $m_{3}$ on the samc side and $t$ (tare) on the other platform. $b$ ) Second operation. The body $\mathrm{K}$ is scparated from the suspension device. The balance is again equilibrated, this time with the mass $m_{2}$, the tare $t$ being unchanged.

The mass $m_{2}-m_{\mathrm{t}}$ has thus been substituted for the apparent mass $m-m_{\mathrm{w}}$ where $m_{\mathrm{w}}$ is the mass of the displaced liquid. A correction must be applied to take account of the air buoyancy on the masses on the left platform, on the one hand, and on the other hand, if the variation in the acceleration of gravity corresponding to the vertical distance between $K$ and this platform. The masses of the auxiliary platforms $P_{1}$ and $P_{2}$ are climinated from the results thanks to a second weighing in which the masses $m_{1}$ and $m_{2}$ are associated with $P_{2}$ and $P_{1}$ respectively. 
The balance developed by the NPL is a classical two-platform balance with three knives; the knives remain in contact with the flats, but under reduced load, during interchange of the masses.

The one studied at the NRLM also has three knives and two platforms, but the latter have two levels. Substitution of the masses is effected on the two levels of the same platform while the knives remain in contact with their flats and under constant load, even without the balance oscillations being interrupted.

The balance studied at the NBS is, on the contrary, a balance having a single platform and two knives (the second platform is replaced by a counterweight); the knives and flats remain in contact and under constant load during substitution of the masses. The second prototype brought out by the NBS, called balance NBS-2 [12], was put at the disposition of the BIPM in 1970 (fig. 5); its permanent installation in an isolating booth was finished in 1972 and verification weighings were soon started. The balance is necessarily operated from a distance. Determinations of prototype Kilograms with this balance began in 1973 after a first improvement of its thermal stability.

The constant load on the knives during the substitution of masses is such as to strictly maintain the contact conditions and to give the balance a remarkable accuracy. Replacing the second platform by a counterweight reduces the number of knives to two, which simplifies adjusting their parallelism; on the other hand, this practically limits the use of the balance to the comparison of kilograms; furthermore, two interchangeable counterweights must be available, one of platinum-iridium and the other of stainless steel, used accordingly in the comparison of prototype Kilograms or of stainless steel Kilograms. To compare a stainless steel Kilogram with a prototype Kilogram, the platinum-iridium counterweight is used.

Figure 6 shows the releasing of the balance, that is, the passage from the rest position (knives and flats separated) to the weighing position; figure 7 indicates schematically the functioning of the mechanism which permits mass substitution with constant loading.

The balance is equipped with a carrier capable of holding six Kilograms and allowing the placement of the selected mass on the platform. It is then possible to compare six standards (or fewer) in all their combinations, two-by-two. As an example, such a comparison of four Kilograms lasts about two and one-half hours. Each comparison of two Kilograms is carried out in terms of the counterweight. One determines, according to the maximum excursions read on a scale, the equilibrium positions of the beam under the following different loads: a) Kilogram A, b) Kilogram B, c) Kilogram B + added weight, d) Kilogram A + added weight, e) Kilogram A. The added weight is a small rider of known mass which serves to calibrate, in terms of mass, the value of one division on the scale. These observations being more numerous than needed for determining the difference between the masses of Kilograms A and B, the statistical uncertainty of this dif- 


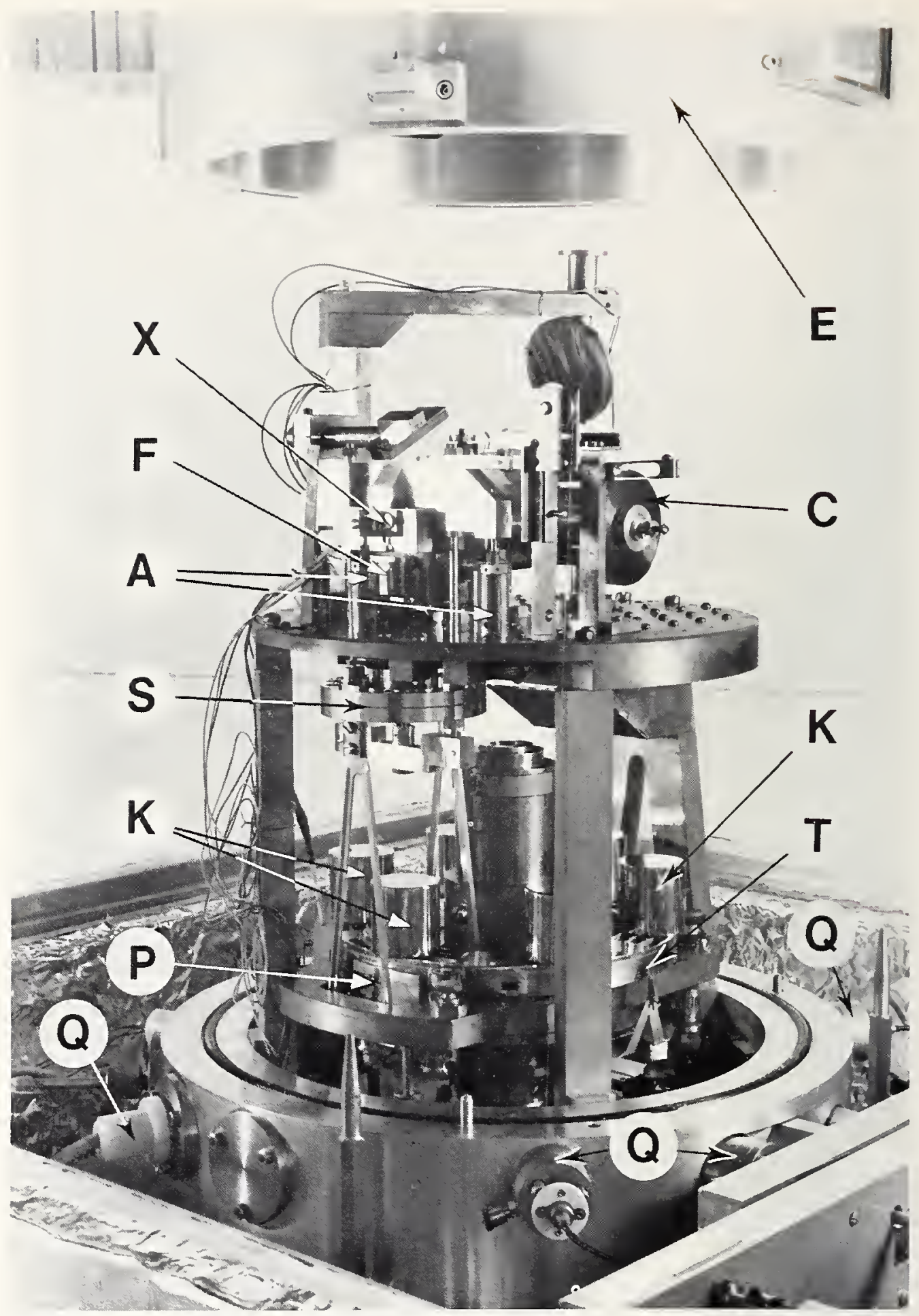

Figure 5. The NBS-2 single-platform balance of the BIP.I. C. counterweight attached to the beam F: X. assembly of crussed plane knives: A. stops for arrcstment and alinement: S. Cardan suspension for platform P: T. circular carrier capahle of holding six Kilograms $\mathrm{K}$ (here, of platinum-iridium) and of placing one of them on P: Q, air-tight passages for the mechanical controls and measming circuits (tempcrature, pressure, humidity). The upper part $\mathrm{E}$ of the air-tight enclosure has been raised and part of the thermal protection removed. 

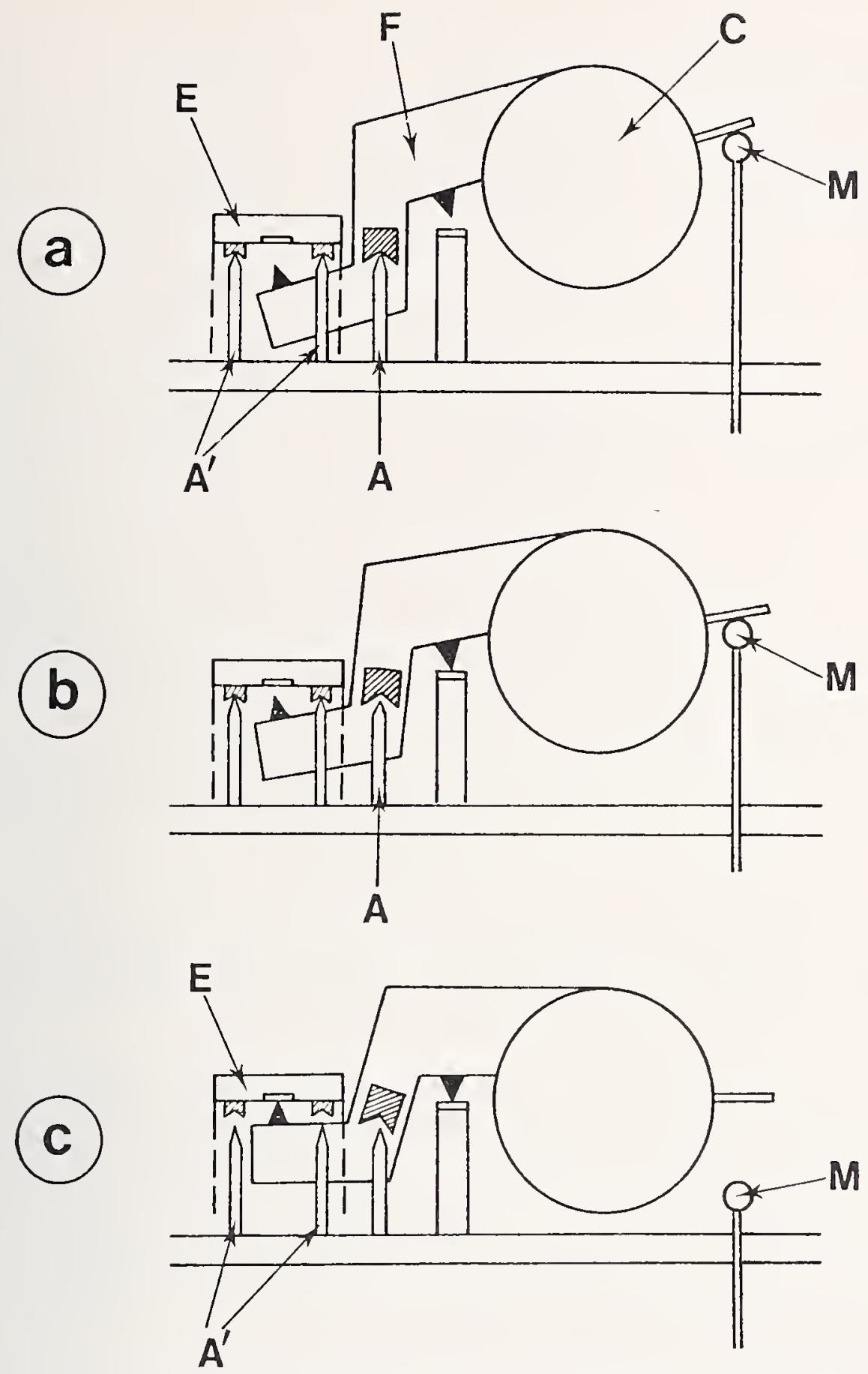

Fiqure 6. Releasing in halance NBS-2. (a) Rest position. The beam F, to which is fixed the counterweight $C$, rests on the movable stop $M$ in its high position, and on the 1 wo alinement stops located on both sicles of the beam. The platform rests on alinement stops A' through its stirup E. (b) Intermediate position. During the first phase of lowering stop M, the principal knife comes into contact with its plane, the beam pivots slighly and no knower rests on stops A. (c) Weighing position. During the second phase of lowering stop $\mathrm{Il}$, the end hnife comes into contact with its plane fixed on the stirrup E of the platform and raises the stirrup which no longer rests on stops $A^{\prime}$. The balance can oscillate freely.

Reverse operation of the movable stop M allows passing from the weighing position to the intermediate position and then to the rest position. 


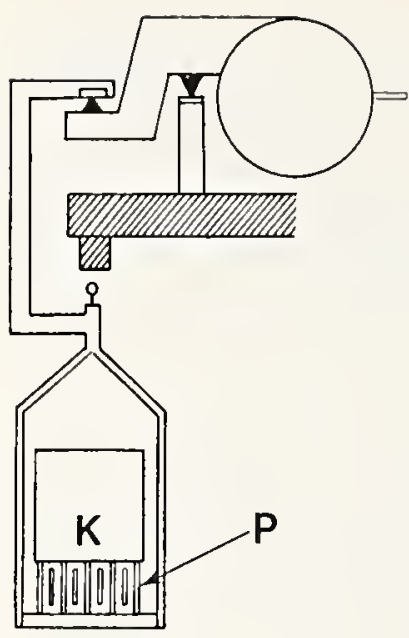

a

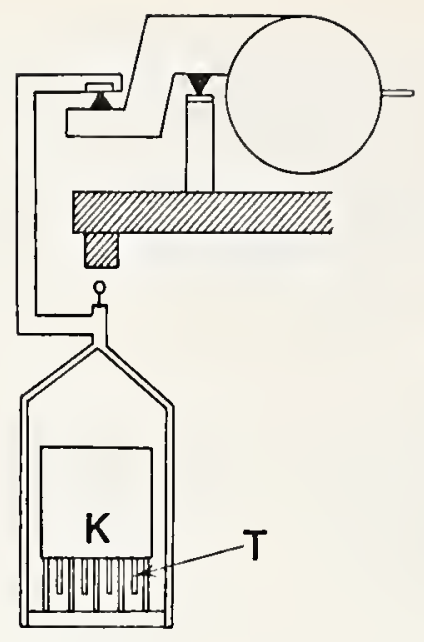

(b)

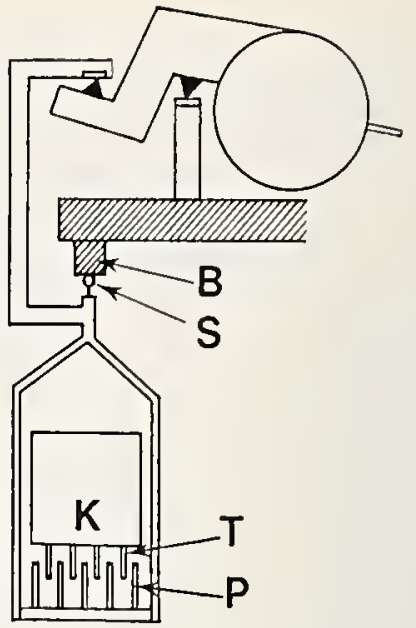

(C)

Figure 7. Balance NBS-2; scheme for substituting masses with constant loading. (a) Wcighing position. The mass $\mathrm{K}$ (Kilogram) rests un the platform $\mathrm{P}$. (b) The carrier ' $\mathrm{T}$ is raised and comes into contact with $\mathrm{K}$. (c) The carrier achieves its lifting motion; the beam tilts slightly until the small sphere $S$ comes into contact with the stop B attached to the balance frame. Carrier $T$ then raises $K$ which is no longer in contact with platform $P$. The loads on the knives thus remain practically constant.

The carrier can then displace $K$ perpendicularly to the plane of the figure and bring another mass in above the platform. In lowering, it places this mass on the platform (position shown in (b)), then returns to the weighing position (a).

ference can be deduced. Various improvements which have been made, especially that giving operation of the balance under an air-tight dome, have allowed reducing the standard deviation ${ }^{6}$ of such a comparison to $2 \mu \mathrm{g}$.

It is thus possible to determine the mass of a prototype Kilogram with a standard deviation of the order of $1 \mu \mathrm{g}$, making only a small number of measurements, under the condition, however, that the masses of the BIPM working prototypes are known with sufficient accuracy. Since this is not so, we foresee a new determination in the near future, relative to 3 , of its six check standards and the working prototypes, followed by the third periodic verification of the prototype Kilograms.

\section{Problems Posed by Improvement of Weighing Precision}

We have already seen that the correction for air buoyancy limits the comparison of a stainless steel Kilogram with a prototype Kilogram.

Formulas used in various laboratories can give corrections for this which differ by $20 \mu \mathrm{g}$. That becomes entirely disproportionate relative to

"There are 68 chances in 100 that the random error, in absolute value, is less than 1 standard deviation, and 99.7 chances in 100 that it is less than 3 standard deviations. 
the reproducibility which can currently be achieved in weighing. It is therefore appropriate to restudy the establishment of formulas giving the density of air as a function not only of pressure, temperature, and relative humidity, but also as a function of its composition, and to study simple and rapid methods for determining this composition. Another method would consist in experimentally determining the air density in the balance case itself or in an enclosure communicating with it. The air-tight case of balance NBS-2 would be well adapted to the latter solution.

We have seen also that, even with the earlier balances, one could measure the increase of mass of a prototype, due to pollution of its surface, over a period of 5 years. We can now measure it over a period of 1 year. In the past, it was shown that it is necessary, before each important series of comparisons, to submit the prototypes to a cleaning followed by washing in steam, but one was not certain of the stability of prototypes after this treat ment. Whereas the systematic measurements made towards 1965 permitted no conclusion, those which were carried out in 1974 have clearly shown that the masses are stable and that the weighings can be carried out without delay (fig. 8).

It is equally known that the importance of wear and pollution of the prototypes is related to their surface condition. It is therefore fitting to improve the polish. The BIPM has used a paste with a diamond-powder base to achieve polishes of excellent quality which could lead to an appreciable improvement in the stability of prototypes.

Thus, despite the imperfections inherent in a material standard, but thanks to precautions taken for its conservation, the International Kilogram has without fail played its role as a basic standard for nearly a century, through the intermediary of its check standards and some of their prototypes.

It would be very satisfying to define the unit of mass as a multiple of the mass of an elementary particle or of an atom, which would constitute an absolute guarantee of stability. Now, although the ratios of various atomic masses can sometimes be determined with an uncertainty less than $1 \times$ $10^{-7}$, the unit of atomic mass (1/12 the mass of the atom of carbon 12$)$ is currently known to only about $3 \times 10^{-6}$. Despite the constant progress realized in this field, there is little likelihood that in the near future one can gain the factor 1000 which would allow considering the adoption of an "atomic" definition of the Kilogram. 

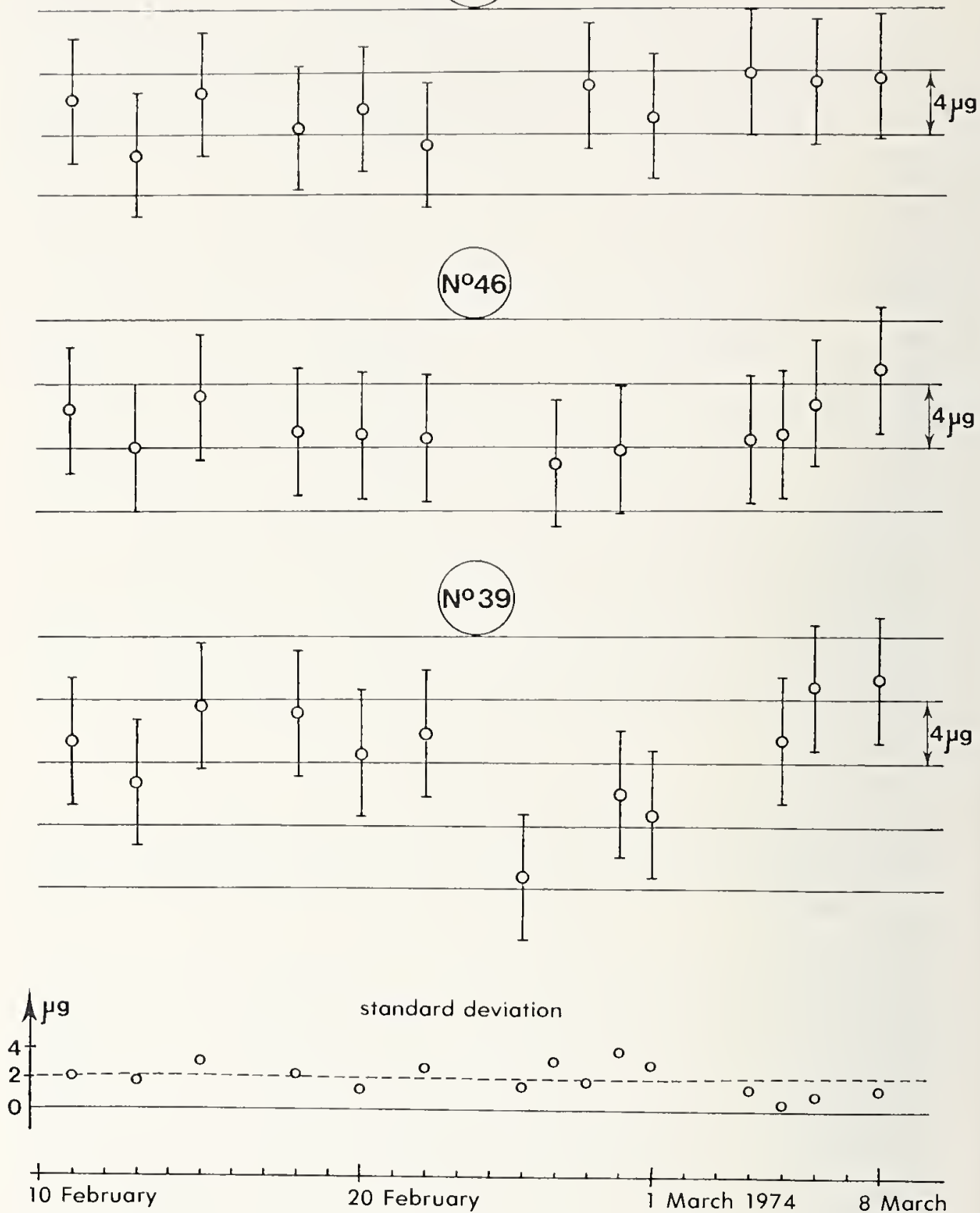

Fiqure 8. Ilistory of the mass of three prototype Kilowrams after a cleaning-washing. and standard deviation of each comparison. With the scheme used for the determination of the mass of each prototype. the standard deviation $\sigma$ of the value of this mass is equal to 0.7 times the mean standard deviation of comparison $(2 \mu \mathrm{g})$, say $1.4 \mu \mathrm{g}$. The error bars correspond to \pm $3 \sigma$, say about $\pm 4 \mu g$.

The figure shows that after a cleaning (henzene and pure alcohol) followed by washing (jet of steam from twice-distilled water). prototypes. Vos. 46 and 52 have not changed significantly during the duration of this study. On the other hand. No. 39 shows an instability probably due to its bad surface condition. 


\section{Bibliography}

[1] Comptes Rendus 5e C.G.P.MI.. pp. 27-33 (1913): Travaux et Mémoires B.I.P.M. 16(1917).

[2] Bonhoure, A., Kilogrammes prototypes, Truvaux et Mémoires B.I.P.M. 22 (3). 82 pages (1966)

[3] Bonhoure, A.. Note sur l'efficacité de quelques procédés de nettoyage des poids en platine iridié. Procès-Verbaux C.I.P.M. 20, pp. 171-178(1946).

[4] Comptes Rendus 10 C.G.P.M...pp. 51-53 (1954).

[5] Marek, W.-J.. Pesées. Travaux et Mémoires B.I.P.M. 1, pp. D53-D58 (1881)(Description of the Rueprecht balance No. 1).

Thiesen, M., Comparaison des prototypes nationaux avec le prototype international 杼, Travaux et Mémoires B.I.P.M. 9, pp. 7-12 (1898) (Description of the Bunge balance).

[6] Guillaume, Ch.-Ed.. Détermination du volume du Kilogramme d’eau (Méthode des contacts. Travuux et Mémoires B.I.P.M. 14,276 pages (1910).

Chappuis, P.. Détermination du volume du Kilogramme d'eau (Première méthode interférentielle). Travaux et Mémoires B.I.P.M. 14,164 pages (1910).

[7] Macé de Lépinay, J., Buisson, H., and Benoît, J.-R.. Détermination du volume du Kilogramme d'eau (Seconde méthode intérferentielle), Travaux et Mémoires B.I.P.M. 14, 128 pages (1910) (Modifications of the Rueprecht balance No. 1, pp. 33-35).

[8] Guillaume. Ch.-Ed., La eréation du B.I.P.M. et son oeuvre, Gauthier-Villars. Paris, pp. 240-258 (1927).

[9] Chappuis, P., Dilatation de l'eau, Travaux et Mémoires B.I.P.M. 13,40 pages (1907).

[10] Broch, O. J.. Poids du litre d'air atmosphérique, Travaux et Mémoires B.I.P.M. 1, pp. A.49-A.58(1881).

[11] Girard, G. and Menaché, M., Variation de la masse volumique de l'eau en fonction de sa composition isotopique. Metrologia 7. No. 3. pp. $83-87$ (1971) and Recueil de Travaux du B.I.P.M. 3, article 13 (1971-1972).

[12] Almer. H. E.. National Bureau of Standards one kilogram balance NBS No. 2. J. Res. Nat. Bur. Stand. (U.S.) 76C, No. 1, pp. 1-10 (1972). 



\section{CHAPTER III}

\section{LENGTH}

One of the principal reasons for creating the BIPM was the unification of length measurements. Considerable work was therefore devoted to the study of the prototype metres and means for comparing them among themselves with the best possible precision. This work resulted in the 1889 definition of the metre, based on the international metre identified by the symbol Af. For almost three quarters of a century, all length measurements made at the BIPM and in many parts of the world used $A$ as the starting point. At the BIPM during this time, the methods of comparison of prototype metres ${ }^{1}$ were continually improved, less costly alloys were studied for the construction of divided scales, the expansion properties of prototype metres and of scales were determined, and methods were developed for the measurement of geodetic wires, end standards, and industrial gages.

In 1895, measurement of the wavelengths of several cadmium radiation lines ushered in a new era. Monochromatic ${ }^{2}$ optical radiations were in fact often used for measuring end-standards by interferometry, and one of them was considered as a transfer device for 1 . Studies of these radiations were uninterruptedly pursued at the BIPM as well as at other laboratories and resulted in the 1960 definition of the metre based on the orange line of krypton 86. Since then, all length measurements must be based on this new standard. This involves major modification of apparatus for measuring material standards, notably scales and geodetic wires. The adoption of the new length standard has not slowed the research on monochromatic radiations, indeed, to the contrary. It is necessary to study carefully not only the st andard radiation itself, its properties and conditions of production, but also other radiations capable of being used jointly with it, in particular laser radiations which will undoubtedly furnish the basis of a new definition of the metre in the near future.

\footnotetext{
1 In this chapter, a platinum-iridium standard of $1 \mathrm{~m}$ nominal length is called prototype metre or metre; a length standard with graduation lines is called a divided scale or scale, and such a standard of nominal $1 \mathrm{~m}$ length is called a metre scale.

${ }^{2}$ These luminous radiations of simple color appear in a spectroscope as narrow lines. In the limit, these are pure sinusoidal waves characterized by their wavelengths in vacuo, or by their frequencies.
} 


\section{Prototype Metres [1]}

In 1889, the lst CGPM ratified "the prototype of the metre chosen by the International Committee" and declared: "This prototype will henceforth represent, at the temperature of melting ice, the metric unit of length." The CGPM thus implicitly defined the metre. ${ }^{3}$ This prototype, the International Metre (AP), has been preserved at the BIPM since that time.

Thirty metres have been constructed. Like $\mathfrak{A I}$, they have the $\mathrm{X}$ cross section calculated by Tresca (fig. 1) and are constituted of an alloy of platinum (90\%) and iridium (10\%). They have been carefully compared among themselves and with $\mathfrak{A A}$, and their expansibility has been determined. Thus these metres, distributed at the time of the 1st CGPM to the countries requesting them, comprised a consistent set of national standards and defined in each country the same length unit with a precision very largely sufficient. The BIPM itself acquired several of them as working prototypes.

To assure the permanence of the uniformity thus realized, the Metre Convention prescribed periodic verifications of the set of national metres.

The first verification, starting in 1921, lasted some 15 years. Indeed, it greatly surpassed the goal of a simple verification and provoked a set of studies whose results considerably advanced the metrology of length. Of special note, it instigated a new determination of the expansion coefficients of the prototype metres. This determination required extremely lengthy studies: the definitive results were finally published only in 1940 .

No other systematic verification of the set of metres has been organized since, but numerous national metres have been individually verified by comparison with the working prototypes of the BIPM, themselves verified relative to $\mathfrak{A t}$.

The platinum-iridium alloy has fully justified the hopes put in it, from the point of view of its stability and inalterability, and the $\mathrm{X}$ section was certainly the best for excellent rigidity and economy of material; the efforts of the BIPM have therefore been directed especially toward improving the engraved lines as well as the flatness and polish of the surfaces carrying these lines. Progress has been constant. Although in the last century one had to reduce the polished surfaces to two small patches of $8 \mathrm{~mm}$ length at

\footnotetext{
${ }^{3}$ This definition was made precise by the 7th CGPM, in 1927, in the following terms: "The unit of length is the metre, defined by the distance at $0^{\circ}$, of the centers of two lateral lines engraved on the bar of platinum iridium deposited at the International Bureau of Weights and Measures, and declared the prototype of the metre by the First General Conference for Weights and Measures, this scale subject to standard at mospheric pressure and supported by two rollers of at least a centimetre in diameter placed symmetrically in the same horizontal plane at a distance of $571 \mathrm{~mm}$ from one another."
} 


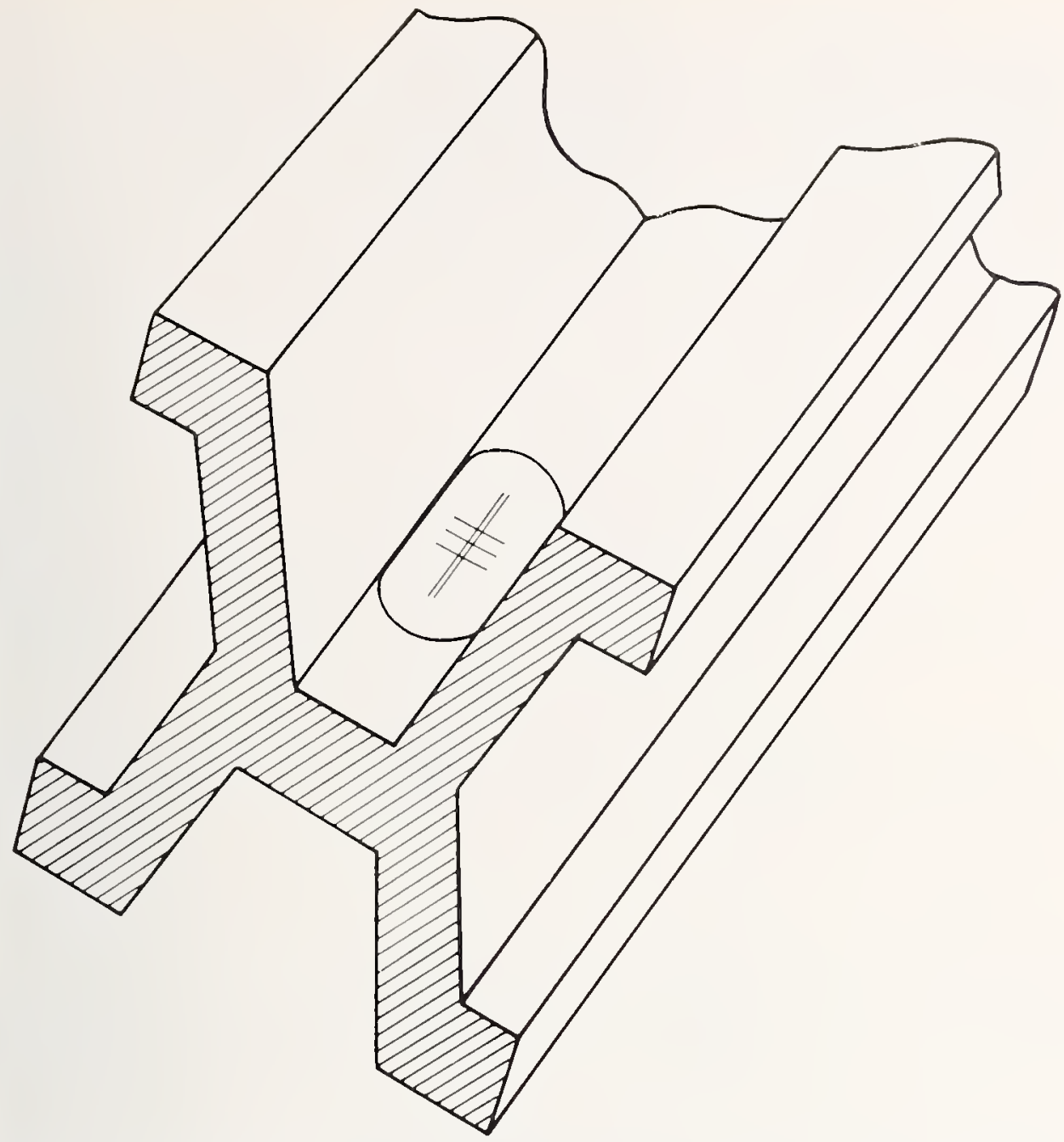

Finure 1. One end of the International Metre. We see the shape of the cross section, inserta. ble in a square having $200 \mathrm{~mm}$ siles. and the 3 lines engraved on a pulished "patch" in the plane of the neutual filers. The distance leetween the middle line and the anadogons line on the patelo at the other end defined the metre when this prototype was at rhe temperature of melting ice. The useful purtion of these limes in delimited by wo longitudinal fielueial linus.

each end of the prototype metres, it is now possible, thanks to the joint experiments of the BIPM and the Geneva Society of Physical Instruments, to put a specular polish on the entire length of the prototypes.

The development of a refined technique for dressing the lines after grooving has also allowed ohtaining lines with sharp straight parallel edges (fig. 2) easy to aline with the microscope cross-hairs. Starting in 1937, all the working prototypes of the BIPM and many national metres were furnished with new lines.

The distance specified between the support rollers is chosen to minimize the shortening of the standard by flexure under its own weight. 


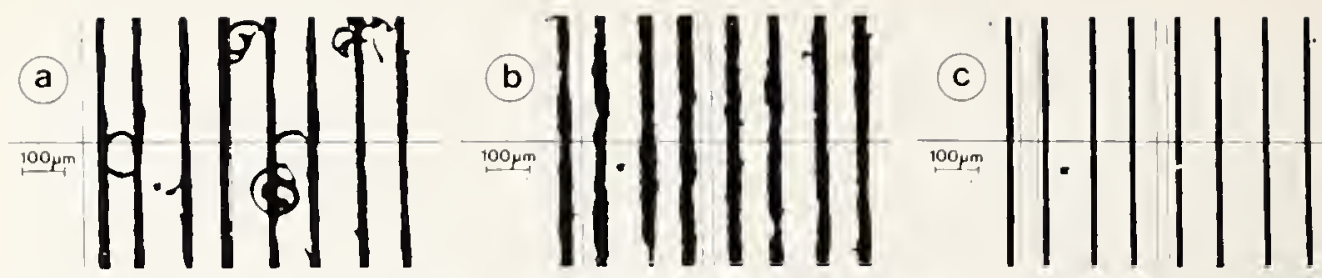

Figure 2. Appearance of lines engraved on a platinum-iridium prototype: (a) before trimming: (b) during trimming: (e) after trimming. The narrow lines are the wires of the reticle of the micrometer ocular.
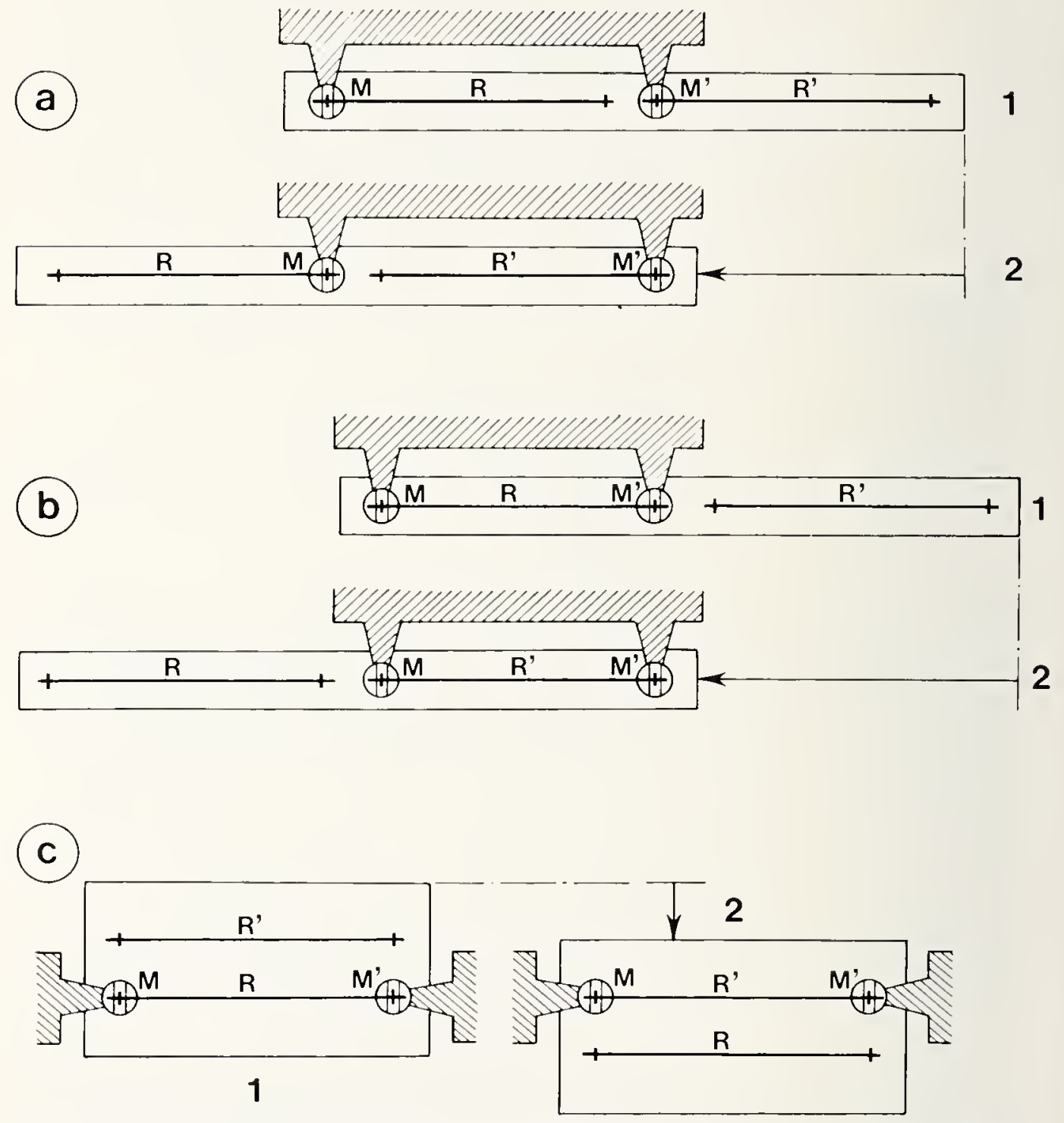

Figure 3. Prineiple of the eomparison of two metre scales. (a) The two mieroscopes $\mathrm{M}$ and $\mathrm{M}$. point the two lines of the same scale before (scale R. position 1) and after (scale R', position 2) the displacemcut which ean he longitudinal (b) or transverse (c). In eases (a) and (b), the space required for the instrument is greater than $3 \mathrm{~m}$ and the displacement is of the order of $1 \mathrm{~m}$; in case (c), the displacemem is only some 10 centimetres. 
The BIPM has also contributed to studies relating to the construction of length standards in metals or alloys less costly than platinum iridium, of lower expansion, or capable of being polished more easily.

The systematic study by Ch.-Ed. Guillaume on nickel steels and the important discoveries of invar and elinvar to which this study led, made his reputation and have largely contributed to the reknown of the BIPM.

The best standards, as regards the quality of their lines, are steel scales covered by a thick electrolytic deposit of nickel. The specular polish which is realized on this metal is excellent, and the lines which can be engraved are remarkable. One thus obtains standards of good stability, very corrosion resistant, and whose lines are very well defined: they are generally divided along their entire length. The cross section of these scales generally has the form of an $\mathrm{H}$ inscribed in a square $24 \mathrm{~mm}$ on a side. This is less economical of metal than the $\mathrm{X}$ section of the platinum-iridium prototype metres, but much easier to construct.

\section{Measuring Instruments}

Two methods can be envisaged for the comparison of two line standards (fig. 3).

In the first, the two standards are placed in a single line with a fixed separation. Two fixed microscopes point simultaneously at the corresponding extremities of the two standards, then, after longitudinal displacement of the set of the two standards, they point at their other extremities. This method achieves the same observation conditions for the lines of each standard but it is seldom employed because of the risk of relative displacement of the two standards and the difficulties of controlling their position. Furthermore, for metre scales, it requires very bulky longitudinal comparators.

The second method consists in pointing at the two extremities of one standard simultaneously with the two microscopes; the two extremities of the second standard are then introduced under the microscopes by means of either a longitudinal or transverse displacement. This last solution leads to transverse comparators of much smaller dimensions.

\section{Standard Comparators}

The comparators [2] used for measuring metres and metre scales were transverse comparators.

The first one used, the Brunner comparator, was installed in 1879; in its primitive form it conforms to what has just been described and did not 
permit observation of the two lines of the same standard under rigorously identical conditions. A basic improvement which was made in 1938 consisted of furmishing it with reversible microscopes, that is to say. microscopes which can be rotated a half-turn around their optical axis.

The combination of observations of the standard in the position called $A B$. and in position BA after rotating the microscopes. is equivalent in effect to an interchange of the microscopes.

To point a line is to frame its image between the two lines of the reticte in the micrometer ocular of the microscope. Each observer. however, performs this operation in a different way: in not framing the line in a completely symmetric way, he commits an error called the error of bisection. This error is eliminated if the observer points the line by approaching it successively from one side and from the other.

These two examples, one of which relates to the improvement of the apparatus and the other to improvement of the operating procedure, illus trate the perpetual concern of the metrologist: seeking and eliminating errors.

The Brunner comparator was replaced in 1954 by a new standard com parator, directly inspired by the former one. This comparator (fig. 4a) rested on its own new foundations to assure perfect stability. Standards being compared were still immersed in a water bath; constant circulation assured crood stability and uniformity of temperature. The tube of each reversible microscope (fig. $4 \mathrm{~b}$ ) was provided with a sliding sleeve whose lower portion, closed by a flat window, was partially immersed during measurement: the lines of the scales could therefore be point ed despite the motion of the water.

Witl this comparator, it was possible to measure the difference between two metre standards with good lines, with a precision of $0.1 \mu \mathrm{m}$.
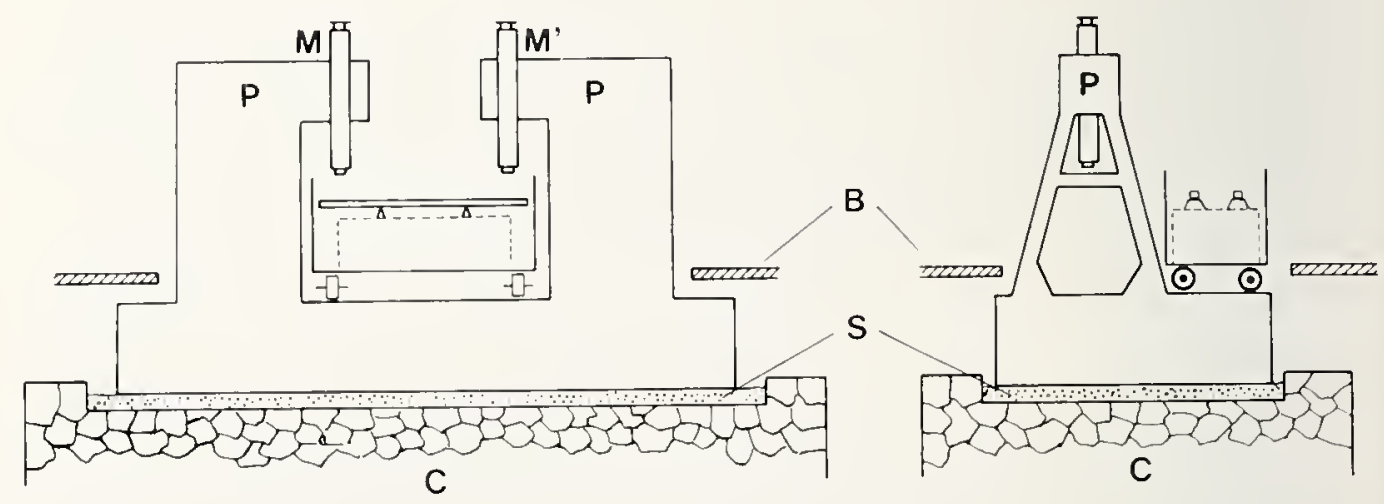

Fioure 4a. The standard comparator. Diagram of the installation. l'. reinforced concrete pillars supporting the microscopes $\mathrm{M}$ and $\mathrm{I}$ ': C. masonry foundation: S. sand layer: B, floor isolated from the comparatur. 


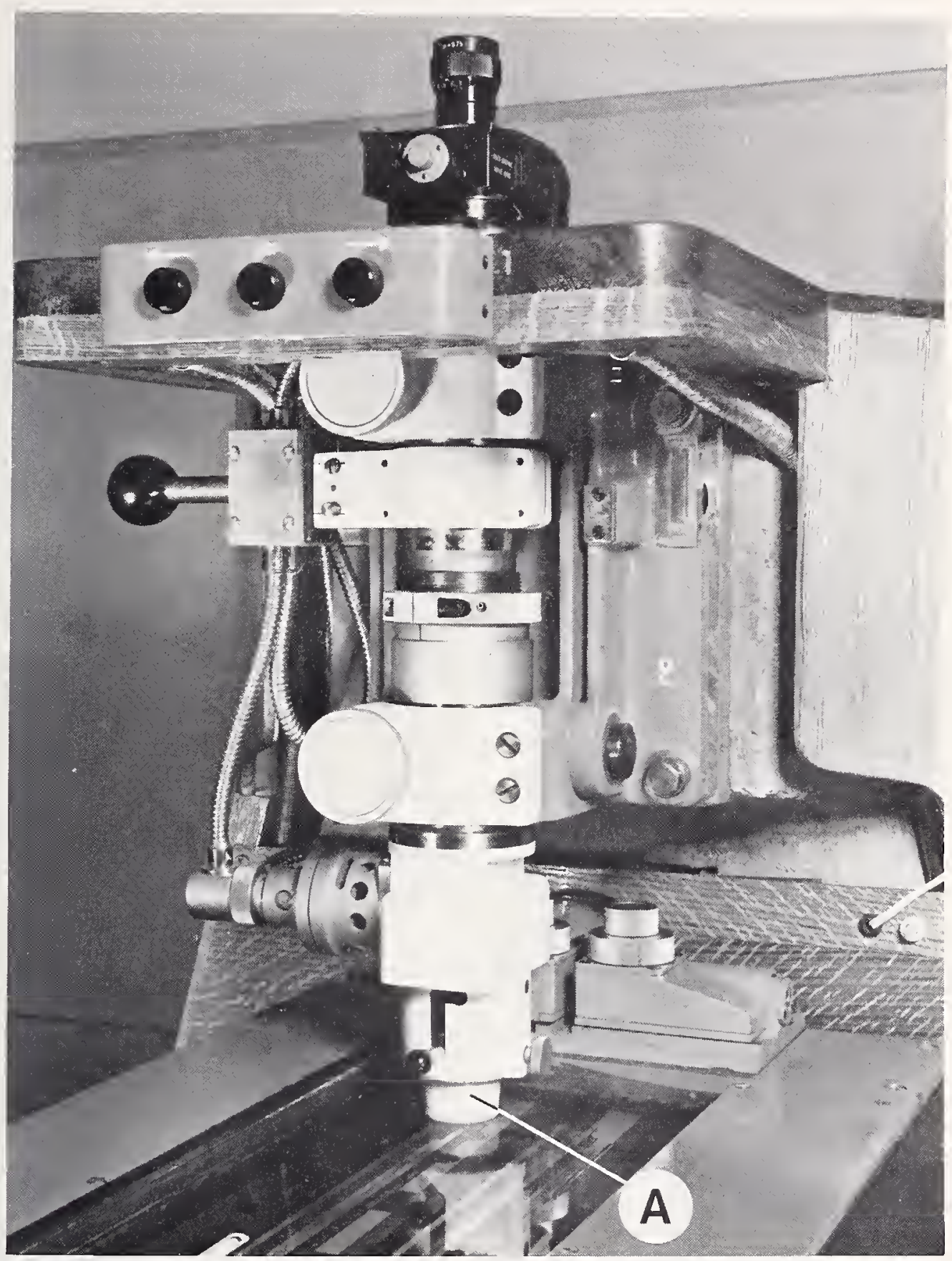

Figure 4l). The standard comparator. The reversible micruscope. Below the sliding sleeve $A$ can be seen the two scales to be compared, immersed in water. 
This transverse comparator, installed in 1925 and improved since, permits comparing the lengths of two scales, one held at a fixed temperature and the other taken to various temperatures spaced betwcen 0 and $40^{\circ} \mathrm{C}$.

Under these conditions, the uncertainty of the determination of a coefficient of mean expansion is about $10^{-8} \mathrm{~K}^{-1}$.

\section{Geodetic Baseline [3]}

The discovery of invar by Ch.-Ed. Guillaume made an important contribution to the success of the method conceived by the Swede Ed. Jaderin for measuring, with the use of a metallic wire under constant tension, the geodetic baselines needed for triangulation of land. For the standardization of these wires, a first "geodetic baseline" was installed at the BIPM in 1900. It was replaced in 1925 by a second baseline which underwent an important transformation in 1949.

The 1925 baseline comprised in fact two distinct parts: a baseline with microscopes or "primary baseline," and a reference baseline or "secondary baseline." The primary baseline consisted of seven microscopes with $4 \mathrm{~m}$ separations between adjacent ones. The six intervals so defined were measured by means of a $4 \mathrm{~m}$ invar scale carried by a moving carriage rolling along a track. The length of the primary baseline was transferred to the reference baseline by means of two 24-metre primary wires.

In 1949, the primary baseline was equipped with removable references. With the references removed, one determines as previously, by means of the $4 \mathrm{~m}$ scale, the intervals between microscopes and consequently the distance between the end microscopes. The end references are then put in place and pointed with the microscopes: the distance between these references is obtained. Comparison of wires with the baseline formed by the references gives their lengths.

The uncertainty in the most common geodetic wires, those of $24 \mathrm{~m}$, was about $10 \mu \mathrm{m}$ toward 1960 .

\section{Toward the Change of the Definition of the Metre}

As early as 1827, the French physicist J. Babinet asserted that it was necessary to look for a length standard in a visible wavelength and not in the dimensions of a material object. Optical radiations, however, are far from having the simplicity that was then attributed to them: their study gave birth to a new science: spectroscopy.

In 1859. J. C. Maxwell had suggested choosing as a natural length standard, the wavelength of the yellow line of sodium which is easily 
produced by introducing a little salt of this metal into a flame which is initially almost colorless. It was observed, however, that this light comprises two radiations whose separation is about a thousandth of the mean wavelength.

Later, the green line of natural mercury was considered. Spectroscopes having become more powerful, it was recognized that this was really a complex group of neighborhood radiations covering a range of about a ten-thousandth of their mean wavelength.

Toward 1890, an American physicist, A. A. Michelson, discovered a radiation, the red line of natural cadmium, whose sharpness and reproducibility were unequalled at the time. In 1892-1893, by means of an interferometer which was specially built and shipped to the BIPM, he measured, in collaboration with J. R. Benoit, the wavelength of this line relative to 毁 [4]. Confirmation of his results was obtained in 1906 by means of interferometric etalons called "Fabry-Perot." 4 In dry air at $15{ }^{\circ} \mathrm{C}$, this wavelength is

$$
\lambda_{\text {Cd }}=6438.4696 \times 10^{-10} \mathrm{~m},
$$

a value which was confirmed later in various repetitions.

By international accord in 1927, it was agreed that the red line of natural cadmium would become the length standard for spectroscopy, the unit of length being the ăngström $(\AA)$ defined by

$$
\lambda_{\text {cd }}=6438.4696 \AA
$$

Thus wavelengths expressed in ăngströms would not be affected in case a new determination of $\lambda_{\text {Cd }}$ relative to the metre should give a slightly different result.

At the BIPM, we did not wait for the definition of the ăngström before using wavelengths for length measurement. Even before J. R. Benoît and Ch.-Ed. Guillaume had made the first attempts to measure the wavelength of the "soda light" in 1884, H. Fizeau had constructed in 1880 an apparatus using the interference of light for measurements of expansion. An improvement on this dilatometer in 1882 allowed making these measurements in vacuo. This apparatus was used on some samples to determine the expansion coefficient of the prototype metres [5].

Interferometric methods are particularly well adapted to the measurement of small length variations and to the measurement of small flat-end standards because the comparison of these standards to line standards is much more difficult. These methods permit direct measurement of the

\footnotetext{
4 The term "Fabry-Perot etalon" applies to a set of two parallel semi-transparent mirrors fixed to the extremities of a very stable structure (a tube or U-shaped channel of invar). Light which enters the etalon along its axis is subjected to multiple reflections which give rise to particular interference phenomena.
} 
distance between the free face of such a standard and the plane which is in contact with the other face of the standard, whether the standard adheres to the plane or is simply placed thereon.

With the use of gages with parallel flat end beginning to expand in industry, A. Pérard construeted for their measurement, in 1920, a first special interferometer using Fizeau fringes (see fig. 11 (a)) and, 3 years later, a second interferometer, similar in principle but allowing the measurement of gages of very great length [6]. This apparatus remained in service until 1971.

As early as 1925, the Michelson interferometer was used at the BIPM for measuring the length and refractive index of six quartz etalons of 10,20 , $30,40,50$, and $100 \mathrm{~mm}$, which were transfer devices for $\mathfrak{A A}$.

The utilization of interferometric methods involved the detailed study of corrections appropriate to these methods, notably the correction for the refractive index of air, permitting the calculation of the true wavelength of a radiation under the conditions of measurement as a function of those conditions (temperature, pressure, humidity) and of the accepted wavelength under reference conditions (for example $15^{\circ} \mathrm{C}$, standard atmospheric pressure, dry air).

This utilization also involved thorough study of the metrological qualities of the radiations themselves. A. Pérard began a systematic study of the radiations of cadmium, mercury, helium, neon, krypton, zinc, and thallium in 1921 [7]. He showed that the wavelength of each radiation studied seemed to vary slightly when different distances were used for the measurement. This effect, which was quite predictable for the blue and green lines of cadmium and for the mercury radiations, which were known to be complex, appeared also for the red line of cadmium, although in a lesser degree. It was natural for A. Pérard to oppose the adoption of this line as the basis for a new definition of the metre after he had thus proved its complexity.

The physical causes of the complexity of optical radiations have since been discovered. On the one hand, a natural element is generally a mixture of several isotopes whose lines do not coincide exactly. The red line of cadmium appeared simple by a fortuitous coincidence. On the other hand, the maynetic properties of the atomic nuclei of certain isotopes confer on their cmitted radiations a structure called hyperfine. The ideal standard could thus be found only among the radiations of a pure (or mononuclidic) isotope whose nucleus is free of magnetism. Theory predicts that such a mononuclide must have an even atomic number $Z$ and an even mass number.

Radiations actually produeed always have a spectral width much greater than their natural width. The principal reason is the Doppler effect: when an atom is approaching the apparatus used for measuring, the observed wavelength is diminished; when the atom is receding, the wavelength is increased. In a gas, atoms are subject to a thermal agitation 
motion; they emit slightly different radiations according to the component of their velocity in the direction of the observer, and the spectral line is broadened. This broadening is smaller for heavy atoms whose thermal speeds are lower at a given temperature. That eliminates helium 4 for example, by reason of its small atomic mass, although its atomic number and its mass number are both even. One is also interested in choosing an element which can emit a spectrum even at low temperature.

Toward 1950, a short time after one knew how to separate isotopes, three nuclides were proposed by several laboratories (IMM, NBS, PTB) for the production of a standard radiation without hyperfine structure: cadmium $114(Z=48)$, mercury $198(Z=80)$, and krypton 84 or $86(Z=36)$.

Some studies were necessary for making the choice among these three nuclides.

The BIPM, which had already measured the wavelengths of the yellow and green lines of mercury 198 and certain lines of the krypton 84 and 86 isotopes, then pursued its studies with the use of the Michelson interferometer, an apparatus continuously in service and to which several improvements had been made successively, notably the replacement of visual observations by photoelectric sensing [8].

Thus not only the wavelengths but also the spectral profiles (see p. 89) of various radiations were determined. At the time of the second meeting of the CCDM (1957), the BIPM could indicate the results of its measurements of the "half-width" of the red line of cadmium 114, of the green line of mercury 198, and of the orange line of krypton 86.

The conclusion of this work and similar studies made in other laboratories was that the choice should fath on the sharpest of the three lines: the orange line of krypton 86 . This could be produced with a minimum perturbation, measurable by other means, in a cold-cathode discharge lamp (fig. 5) whose capillary is chilled to the temperature of the triple point of nitrogen $\left(63.15 \mathrm{~K}\right.$ or $-210^{\circ} \mathrm{C}$ ); furthermore, it suffers little from self-absorption which would distort its profile, and its luminance is sufficient.

The CCDM had recommended that the wavelength of the radiation chosen be measured by comparison with that of the red line of natural cadmium produced in a Michelson lamp conforming to international specifications, and that a formula established by Edlén [9] be used to go from the wavelength in air to the wavelength in vacuo.

Such are the elements that served to fix the numerical value of the new definition of the metre ratified by the 11th CGPM on October 14, 1960. in the following terms:

"The metre is the length equal to 1650763.73 wavelengths in vacuum of the radiation corresponding to the transition between the levels $2 p_{10}$ and $5 \mathrm{~d}_{5}$ of the krypton-86 atom."

\footnotetext{
5 The energy levels of the krypton-86 atom which occur in this definition are specified according to the semi-empirical designations used by Paschen as early as 1917.
} 


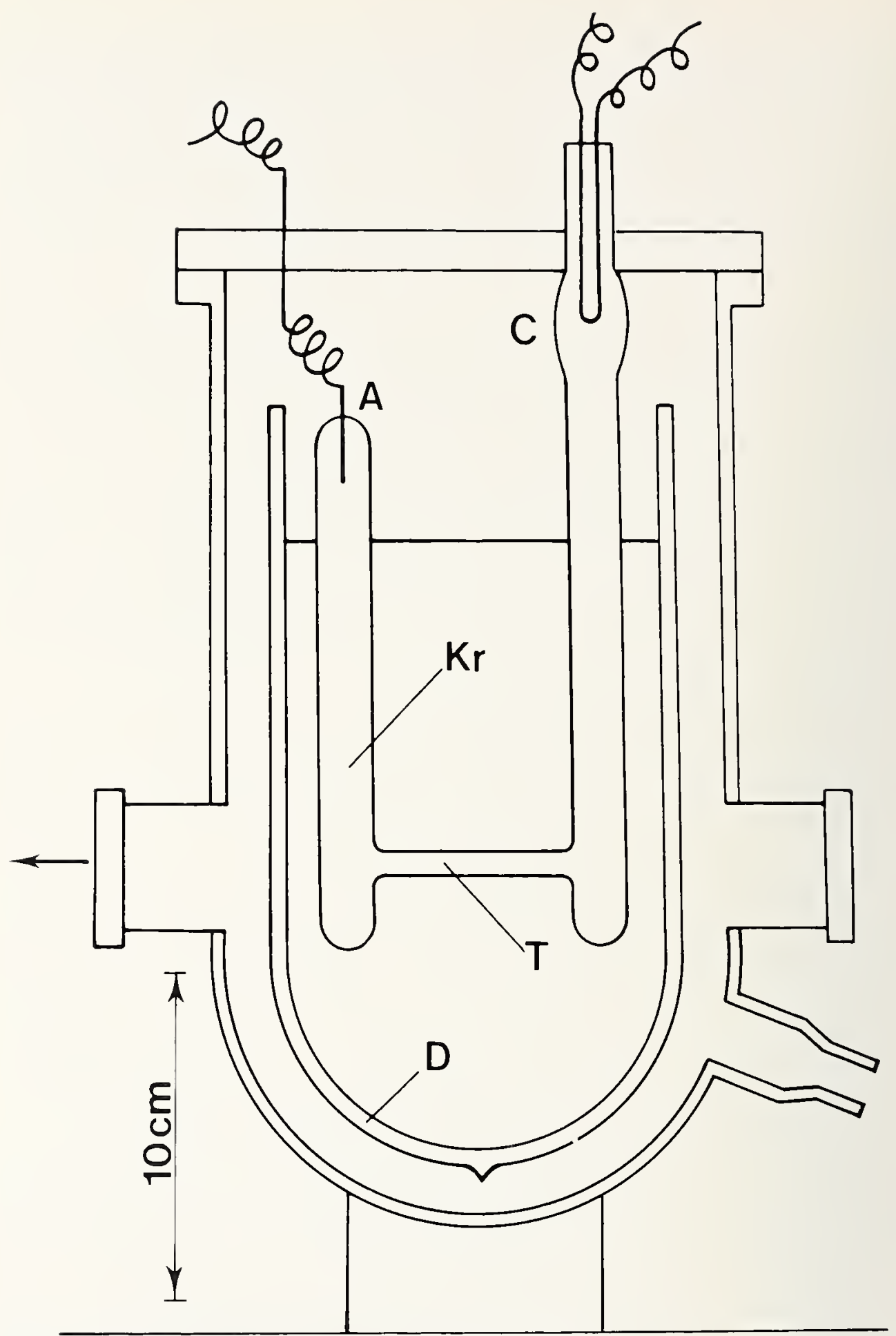

Figure 5. Krypton-86 lamp in its cryostat. A. anode; C. cathole heated by an electric current.

The lamp $\mathrm{Kr}$ is immersed in liquid nitrogen contained in the Dewar flask D. The whole is in an airtight chamber in which there is a partial vacuum for obtaining the triple point of nitrogen $\left(-210^{\circ} \mathrm{C}\right)$. The krypton pressure is the vapor pressure of solid krypton at this temperature. The capillary tube $T$ has a length of about $7 \mathrm{~cm}$ and an inside diameter of about 2 $\mathrm{mm}$ : the electric current density is adjusted to $0.3 \mathrm{~A} / \mathrm{cm}^{2}$. Under these conditions. the orange radiation emitted in the direction of the arrow has a wavelength equal to that which corresponds to the definition of the metre within $1 \mathrm{in} 10^{8}$. 
This definition has been completed by a Recommcndation of the CIPM which specifies the characteristics of the discharge lamp producing the standard radiation [10]. The (rounded) value of the wavelength of this radiation is:

$$
\lambda=1 \mathrm{~m} / 1650763.73=0.605780211 \mu \mathrm{m} .
$$

\section{Putting the 1960 Definition of the Metre Into Practice}

The adoption of a new primary standard of length did not diminish the interest in material standards of length, scales, gage blocks, and geodetic wires; but since then it has been necessary to measure these as directly as possible by comparison with the wavelength of the standard radiation of krypton 86. There were progressively put into service a photoelectric interferometric comparator for measuring divided scales and long gage blocks, an interferometer for measuring gage blocks up to $250 \mathrm{~mm}$ in length, and an interferometric method for measuring the geodetic baseline.

\section{Photoelectric Interferometric Comparator}

Starting in 1952, the BIPM studied, in collaboration with the constructor, a design for a comparator using photoelectric microscopes. These microscopes of a new type had already been proven as essential components of a dividing engine and also in a comparator for calibrating divided scales. The design was improved over several years; the new comparator should permit not only comparisons of scales and calibration of their subdivisions, but also the measurement of ruled scales and end standards up to $1 \mathrm{~m}$ long by comparison with wavelengths of optical radiations. The comparator was installed in 1961 in a specially fitted-out room; it was put in service in 1964 [11] after installation of devices for ancillary measurements (measurements of refractive index and temperature of the air), and putting the photoelectric microscopes into working order. A device called a fringe-counter was adapted to the comparator in 1969 [12].

The comparator must be shielded from temperature variations which would change its own dimensions and those of the standards being measured, and from variations of atmospheric pressure, which would change the refractive index of the air and consequently the wavelengths of the optical radiations used as references; it must also be protected from ground vibrations, which would disturb its operation and the observation of interference fringes. For this, it is enclosed in an air-tight isolating tank of $25 \mathrm{~m}^{3}$ volume mounted on a reinforced $40 \mathrm{Mg}$ platform resting on a dozen concrete pillars. We have achieved an anti-vibration suspension by interspersing between the platform and each pillar a set of helical springs and 


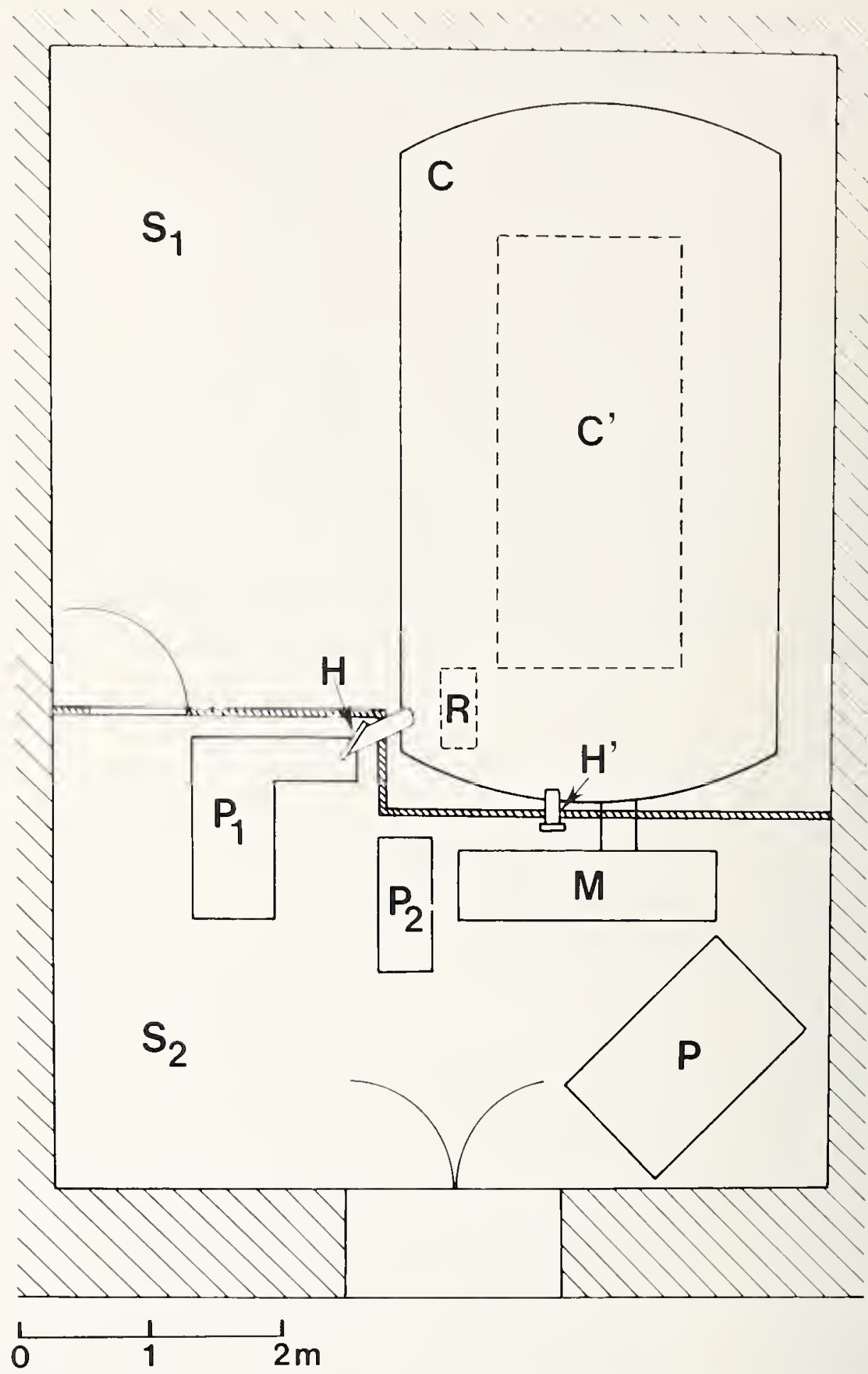

Figure 6. Overall plan of the room for the photvelectric interferometric comparator. $S_{1}$, airconditioned part: $S_{2}$, control room: C. tauk bedded on its anti-vihration platform, and containing the comparator $C^{\prime}$ and the refractometer $R$; P', control and measurement console: $P_{1}, P_{2}$, pillars mounted on the platform. particularly used for supporting light sources: VI, console of comparator motors. mounted on the platform. and used to support the exit telescope of the interferometer: H, H', ports for the entry and exit of light heams. For more details on the optical lay-out, see figure 9. 
a damping device filled with silicone oil. The room (fig. 6) is divided into two parts by an isolating partition. Access to the part containing the comparator can be had only outside the time of measurement and, of course, the tank is entered only for placement of the standards under study. This part of the room is held at a constant temperature, generally $20^{\circ} \mathrm{C}$. to within about $0.1{ }^{\circ} \mathrm{C}$. The light source, comparator motors, control console for the various moving parts of the comparator, and the display of data from the photoelectric microscopes are grouped in the second part of the room, occupied by the operators during measurement.

The photoelectric microscopes operate on the following principle. A rectangular spot $(8 \mu \mathrm{m}$ wide, approximately the groove width, and of adjustable length, for example $160 \mu \mathrm{m}$ ) is projected onto the ruled surface of the scale to be measured. The spot explores a small region by an oscillatory motion along the direction of the scale. The reflected light is received by a photoelectric sensor. If the explored zone carries a groove, the sensor gives a signal at each passage of the spot over the groove. When the groove is centered on the average position of the spot, these signals are separated by equal time intervals, but this is not so if the groove is off center (fic. 7). These signals are electronically processed and the position of the groove relative to the average spot position as reference is exhibited directly on a display device. The resolution of the system is $1 \mathrm{~nm}$ for the measuring range most commonly used $(10 \mu \mathrm{m})$.

(a)

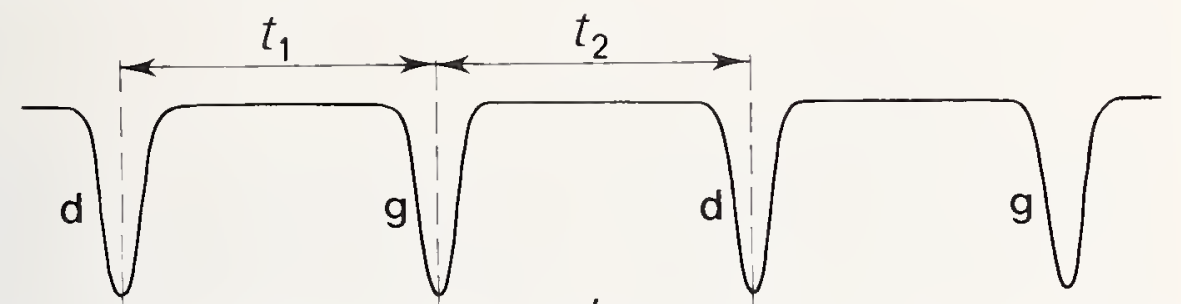

(b)
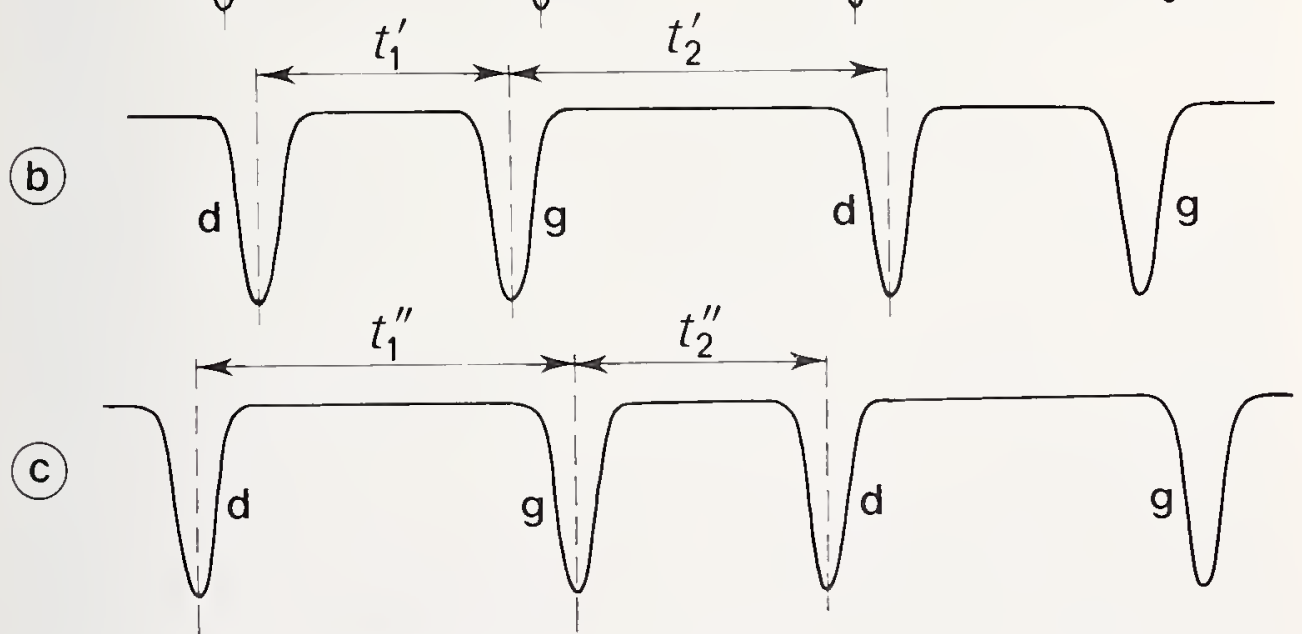

Figure 7. Principle of spotting the position of a line with a photuelectric microscope. Signals delivered by the photoelectric sensor in the three following cases: a) line centered, $t_{1}=t_{2}$ : (b) line decentered to the right, $t^{\prime}{ }_{1}<t_{2}^{\prime}$ : (c) line decentered to the left. $t^{\prime \prime}{ }_{1}>t^{\prime \prime}{ }_{2}$. The signals are produced by the passage of the spot across the line during its movement lowarts the right (signals d) or towards the left (signals g). 
The comparator (fig. 8) comprises two photoelectric microscopes whose positions are adjustable along a common longitudinal beam. Two $1 \mathrm{~m}$ standards can be placed end-to-end on the bench which holds the scales. They are compared by the scheme indicated in figure 3 (b), that is to say, by alternatively pointing the two tines of one, then of the other, by a longitudinal displacement of the scale bench which is supported on a carriage. Naturally, several pointings are made on each standard and its temperature is measured. A complete comparison is very time consuming: it requires the interchange of standards, turning them end for end, and a halfturn rotation of the microscopes about their axes. This method of comparing metre standards or intervals on a single scale has been abandoned, lit tle by little, in favor of interferometric methods; the latter are more rapid and especially more precise because they allow direct comparison of the length of the standard with the primary standard wavelength of krypton 86.

The comparison of line-standards of length with wavelengths of optical radiations requires the comparator to be equipped with an interferometer (fig. 9 (a)). A Michelson interferometer is used here. The parallel beam from a monochromatic source is split into two beams, one of which reflects from a fixed mirror, the other from a mirror moving with the scale. The beams recombine and produce an interference pattern in the focal plane of the

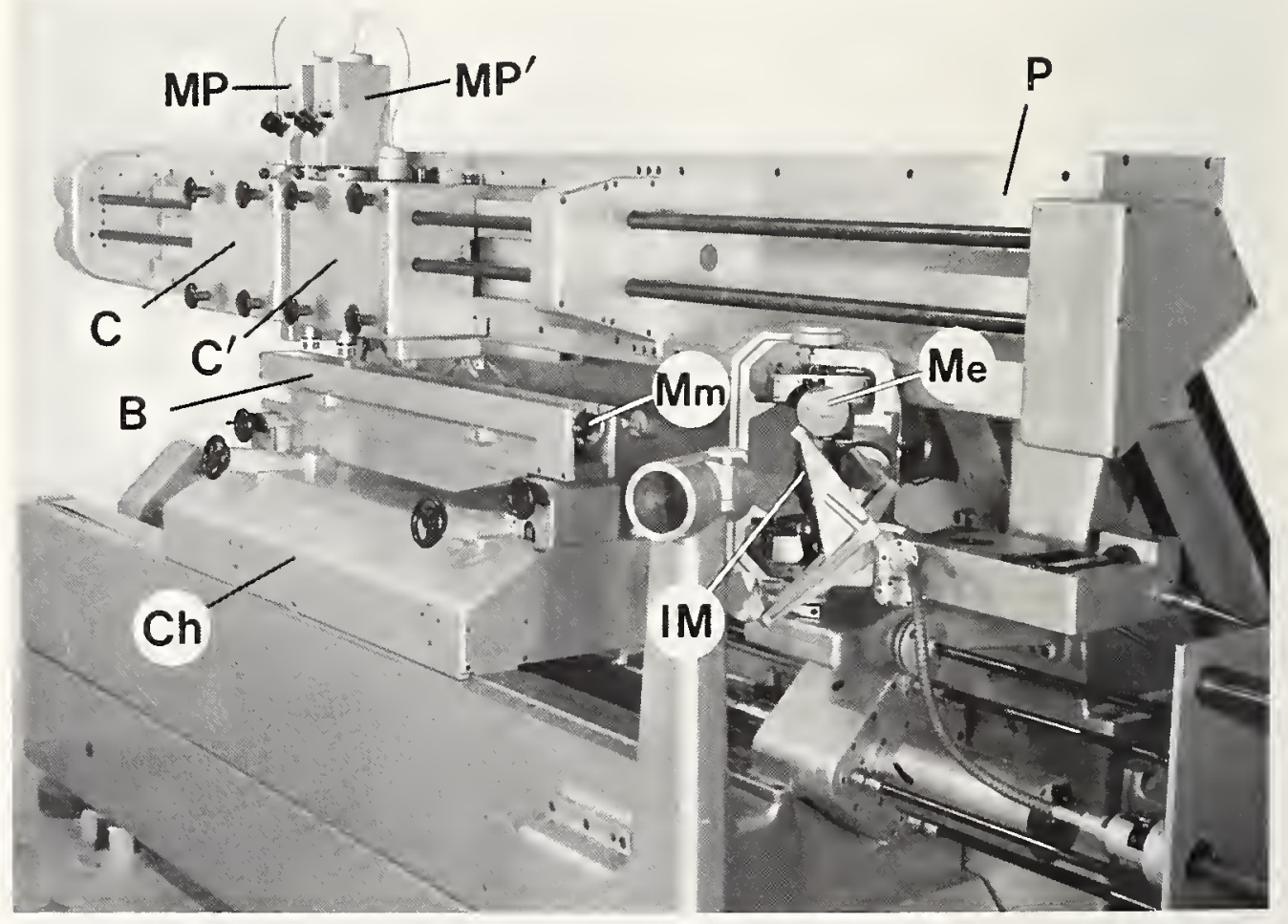

Figure 8. Photoelectric interferometric comparator of the BIPM. B, scale bench: Ch, carriage: $C, C^{\prime}$, shders carrying the photoelectric microcopes MP. MP'. and whose positions are arljustable along the beam P: Mm, nuvable nirror hinked to the scale: Me, eclipsing mirror; IM. splitting and compensating plates of the Vichelson interfermmeter. 
telescope objective. The illuminance at the center of this pattern varies sinusoidally during displacement of the moving mirror; a period of its variation (also called a unit variation of the interference order) corresponds to an increase in the length of the variable beam equal to the wavelength of the radiation used, that is, it corresponds to a mirror displacement of a half wavelength. The principle of the measurement is thus very simple. The initial position of the scale is chosen such that its origin line is centered exactly under one of the microscopes; the scale is displaced so that its end line is centered under the same microscope. To obtain the length $l$ of the scale, equal to the displacement thus made, which is also that of the movable mirror, it suffices to know the wavelength of the radiation used and to determine the variation $p$ of the interference order; we have, in fact, $l=$ $p \lambda / 2$. It is easy to obtain the fractional part of the interference order for the initial and final positions of the scale by measuring the illuminance obtained at the center of the pattern when the length of the variable beam is artificially changed by small known amounts. One thus obtains the fractional part $f$ of the variation of interference order $p$. Two methods are used for determining its integral part $e$.

To apply the first, called the method of excess fractions, one uses several radiations of well known wavelengths, $\lambda_{1}, \lambda_{2}, \lambda_{3} \ldots$ and one measures the corresponding fractional parts $f_{1}, f_{2}, f_{3} \ldots$. The integral paits $e_{1}$, $e_{2}, e_{3} \ldots$ are not known exactly, but from the fact that one must have $l=\left(e_{1}\right.$ $\left.+f_{1}\right) \lambda_{1} / 2=\left(e_{2}+f_{2}\right) \lambda_{2} / 2=\left(e_{3}+f_{3}\right) \lambda_{3} / 2=\ldots \ldots \ldots \ldots \ldots$ and that $l$ is known in advance to a few micrometres, or even closer, one of the integral parts, say $e_{1}$, can be predicted to within 10 units. Then $l$ has 20 possible values, regularly spaced. 'To each of these corresponds a calculated value $f_{2}^{\prime}, f_{3}^{\prime}, \ldots$. . . which is compared with the observed value $f_{2}, f_{3} \ldots$. If the wavelengths used have been well chosen, a single set of calculated values coincides with the set of observed values within the limit of measurement error. The corresponding value of $l$ is the value sought. This method is very reliable with four radiations for the measurement of intervals up to $600 \mathrm{~mm}$. Above that, the determination of fractional parts becomes too uncertain, even impossible with ordinary optical radiations, by reason of the rapid diminution of the visibility of the interference due to the necessary increase of path difference (see p. 91). For a 1-m scale to be measured in this way, it must have a line near the middle: its two halves are then measured successively.

The second method consists in counting the cycles of variation of illuminance at the center of the interference pattern, that is to say, the interference fringes which march through this point. This method had already been used at the BIPM very long ago in 1892 to measure the wavelength of the cadmium red line. At that time, interference patterns were observed visually; counting some thousands of fringes, corresponding to a length measurement of the order of a millimetre, constituted a formidable task. This method has been restored to respectability thanks to the exceptional properties of lasers (see p. 92). The extreme sharpness of laser lines allows 
a
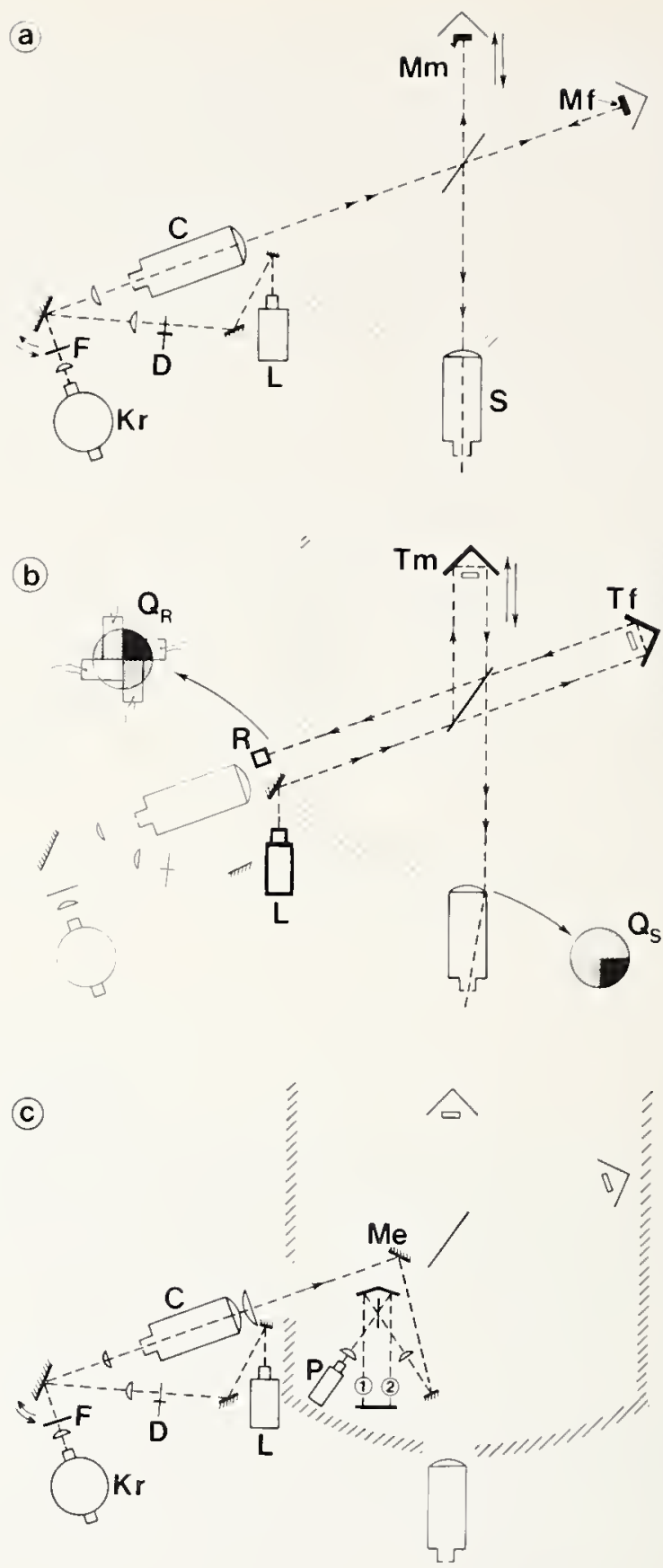

Figure 9. Optical system of the three interferometric arrangements used on the comparator. (a) Michelson interferometer illuminated by the krypton lamp or by the laser: (b) fringe counter for use with the laser; (c) refractomcter (krypton lamp or laser); the vacuum chamber, which can be placed in beam 1 or in beain 2 ; is not indicated.

C, cntrance collimator; D, rotary diffuser; F, interference filters allowing separation of certain lines emitted by the lamp Kr (krypton 86); L, laser: Me, eclipsing mirror; Mf, fixed mirror; Mn, movable mirror: P, refractometer photomultiplier, $Q_{R}$, section of the beam falling on the four sensors: $Q_{S}$, section of the beam observed at the interferometer exit: $R$. array of four sensors: $\mathrm{S}$, exit telescope: Tf, fixed corner reflector; Tm, movable corner reflector. 
obtaining interference without appreciable loss of visibility for all path differences usable in the laboratory. The intensity of laser rays and the use of photoelectric sensors make rapid electronic counting feasible. The device installed on our comparator allows counting at about $6 \mathrm{~mm} / \mathrm{s}$ maximum speed of translation of the carriage. The counting of about 3160000 fringes, corresponding to a length of $1 \mathrm{~m}$, is thus carried out at a rate of 19000 fringes per second. To achieve this performance, we had to add a corner-reflector interferometer to the plane-mirror interferometer (fig. 9 (b)) to maintain proper behavior despite the small defects in the movement of translation. The beam is split among four quadrants by means of retardation plates on one of the surfaces of the stationary corner reflector; these plates are arranged so that the path differences in successive quadrants are $\mathrm{D}, \mathrm{D}+\lambda / 4, \mathrm{D}+\lambda / 2, \mathrm{D}+3 \lambda / 4$. A sensor is associated with each quadrant. Then the signals from two adjacent sensors are in phase quadrature; those from opposite sensors are in phase opposition. By associating these latter signals with two differential amplifiers, the two quadrature signals needed for reversible electronic counting are available.

The speed of this method is particularly important for the calibration of divided scales.

The two methods which have just been described are also applicable to the measurement of flat-end gages. A gage adhering to a base plane constitutes a system of two mirrors (this plane and the free face of the gage) whose separation is the length of the gage. In the method of excess fractions, these two mirrors play the same role as the initial and final positions of the mirror Mm in the case of scale measurement (fig. 10 (a)). Measurement of the fractional parts corresponding to the plane or to the free face are made by selecting one or the other by means of diaphragms suitably located in the beam. In the fringe counting method (fig. 10 (b)), these two mirrors play the same role as the rulings on the scale, and the image of the fixed plane mirror given by the splitter plays the role of the microscope. A displacement equal to the length of the gage is realized by successively placing these two mirrors in the position of the image of the fixed mirror; in the neighborhood of this position, the coincidence can be adjusted by observation of the characteristic white light interference.

The comparator operates in air which is near atmospheric pressure. The wavelength to be used in the formula $l=p \lambda / 2$ must be derived from the vacuum wavelength and the refractive index, $n$, of air by the relation $\lambda=$ $\lambda_{0} n$. The definition of the metre in fact furnishes the vacuum wavelength of krypton 86; the wavelengths of other radiations used are deduced from this by comparison in vacuo. The index $n$ is measured inside the tank by means of a refractometer (fig. 9 (c)). For the value obtained to indeed be that of the index of the air traversed by the beams of the main interferometer, it is 

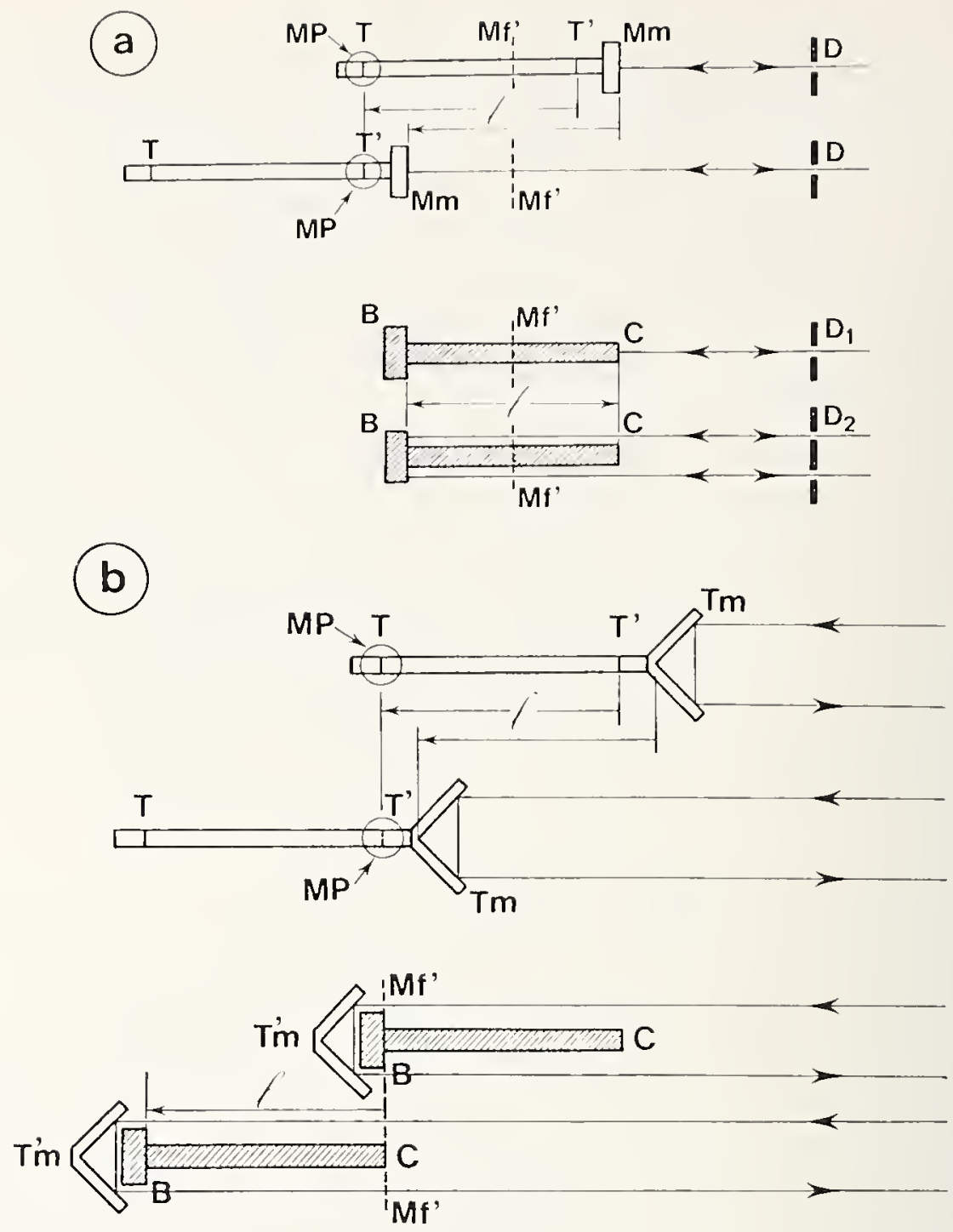

Figure 10. Analogy between measurement of a scale and measurement of a gage-block. (a) Measurement of a scale by the method of excess fractions, or static measurement of a gageblock. The displacement which causes the lines $T$ and 'T" to be centered under the photoelectric microscope MIP successively, is equal to the length $/$ of the scale. The distance between the initial and final positions of the mirror $\mathrm{Mm}$, linked to the scale, is measured by means of interpolation of interference fringes obtained with several radiations for each of these positions (in addition to that, we know that the positions are symmetric about If . the image of the fixed mirror of the interferometer). The free face C of the gageblock, and its base plane B, play the same role as the two positions of $\mathrm{Mm}$. Measurement of the distance requires successive use of two diaphragms $D_{1}$ and $D_{2}$ to limit the useful beams although only one, $D_{1}$ is used for the scale. (b) Nleasurement of a scale or of a gage-block by fringe counting. The translation of the scale is the same as before. The displacement of the movable corner reflector Tm. linked to the scale. is measured by counting interference fringes using laser radiation. The base plane B of the gage-block and its free face $\mathrm{C}$ play the same role as the scale lines $T$ and T' but they cannot be pointed with the microscope MP; to place them successively in the same position, they are made to coincide with Mf", the image of the fixed interferometer mirror. The displacement of the corner reflector Tm', linked to the gage-block, is equal to the length of the latter and is measured by counting fringes as in the case of the scale. 
necessary that conditions, especially pressure, be the same for the two apparatuses; in particular, they must be in the same horizontal plane. This refractometer is itself a small interferometer comprising a vacuum chamber of known length which is placed in one beam, then in the other. The index of refraction is derived from the difference between the interference orders corresponding to these two positions.

The result of the measurement of a standard, scale, or gage, is always adjusted to a reference temperature, for example $20{ }^{\circ} \mathrm{C}$. For this, it is necessary to know the exact temperature of the standard at the moment of measurement, and also its coefficient of expansion. The temperature measurement is made in two steps: a platinum resistance thermometer gives the temperature of a copper reference block located inside the tank, and thermocouples give the temperature difference between the block and the standard itself. The accuracy of this measurement is $0.001{ }^{\circ} \mathrm{C}$. As for the coefficient of expansion, this is determined by measuring the length of the standard at various temperatures over a range of several degrees on each side of the reference temperature; the uncertainty of this coefficient does not exceed $5 \times 10^{-9} \mathrm{~K}^{-1}$.

The uncertainty in the length of a standard at its reference temperature is largely due to the quality of the standard itself: quality of the rulings and flatness of the ruled surface. for a scale; flatness and parallelism of the faces, for a gage block. The uncertainty is generally several hundredths of a micrometre.

Since this comparator was put in service, numerous standards have been measured, including 10 national metres and the 4 working prototype metres of the BIPM. These latter, already known relative to $\mathfrak{A A}$, thus are also known relative to the new definition of the metre. From this is deduced the difference between the "1889 metre" and the "1960 metre." The result obtained has been verified by direct measurement of 19 against the standard radiation. This difference, which is $0.25 \mu \mathrm{m}$ (the current metre being the shorter one) is without practical consequence.

\section{Interferometer for Measuring Gages}

In 1971, the BIPM acquired an interferometer for measuring gage blocks. This commercial instrument replaced the Fizeau-fringe interferometer which had been in use since 1923. Fizeau fringes are formed between two nearly parallel plane mirrors and are observed through one of them which is semi-transparent; these are fringes of equal width. In measuring a gage block (fig. 11 (a)), the length of the air path above the base plane is necessarily longer than that above the free face. This results in a fringe system observed on the base plane which has less contrast than that seen on the free face. This reduction of contrast limits the length of gages 


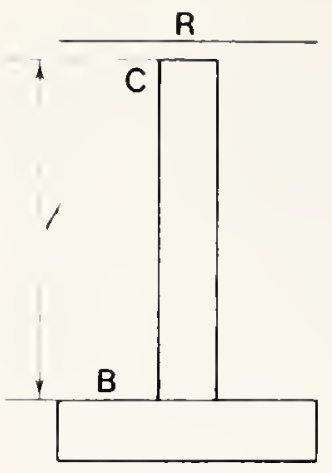

a

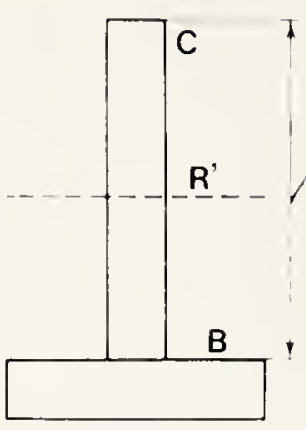

b)

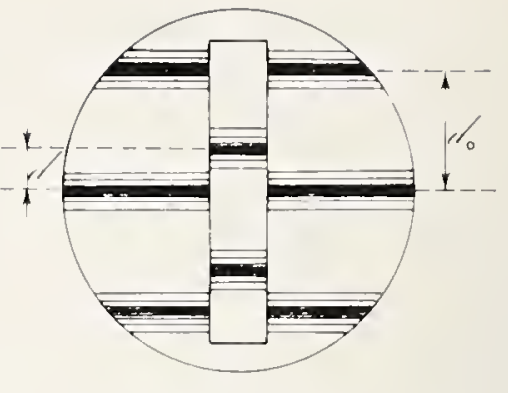

C

Figure 11. Static measurement of gage-blocks. (a) With a Fizeau interferometer, (b) With a Michelson interfcrometer.

The measurement of the length / results from interpolating the equal width fringes which are formed between the reference plane $R$ (semi-transparent mirror) or $R$ ' (image of a fixed mirror) and, on the other hand. the free face $C$ of the gage-block or its base plane B. (c) Appearanee of the fringes ubserved in case (b). The interpolation consists in lncating the position of a fringe given by $\mathrm{C}$ in relation to the $\mathrm{two}$ fringes. given by $\mathrm{B}$. which enclose it. The excess fraction here is $/ / d_{0}$.

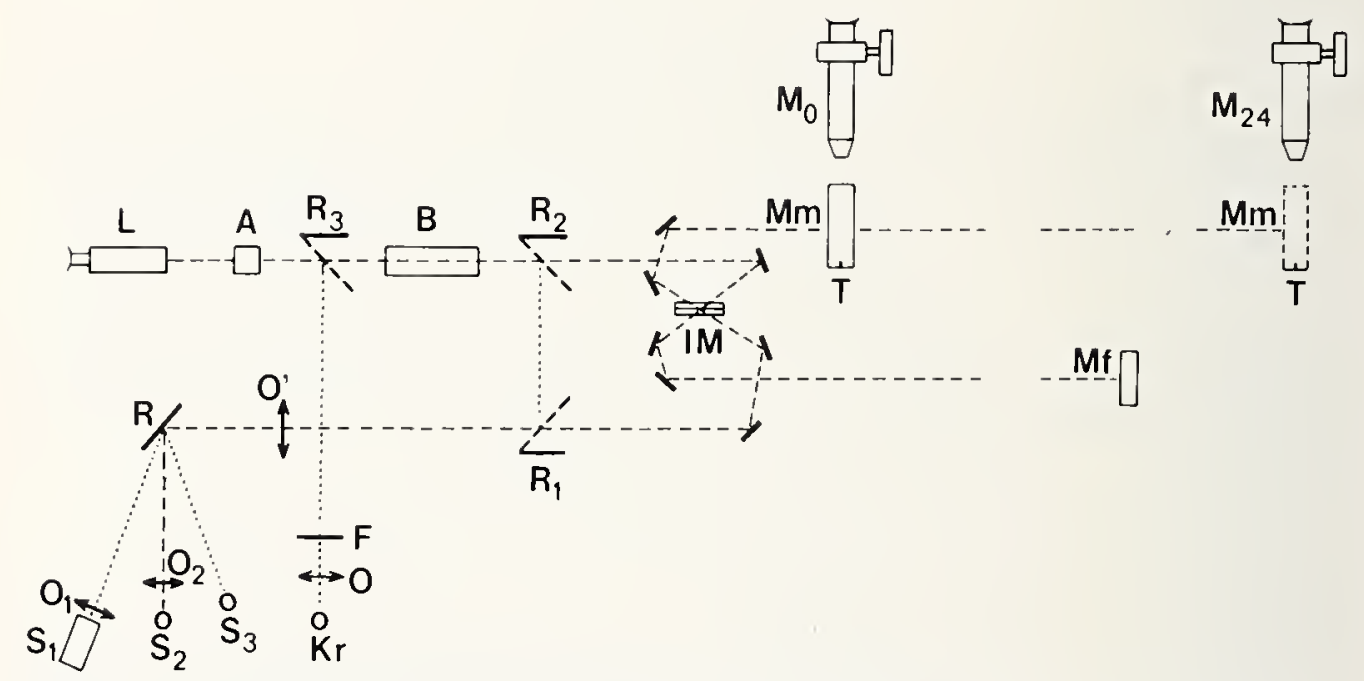

Figne 12. I'lan of the arrangement for interferometric measurement of the 24 -metre gendetic baseline. A, B, Fabry-l'ernt etalons of $0.25 \mathrm{~m}$ and $1 \mathrm{~m}$ : F, interference filters permitling the selection of certain radiations from the lamp Kr (hrypton 86). for the measurement of 1 (mirror $R_{3}$ in its homer position): $S_{1}$, helium-nemn laser, and $S_{3}$, point source, used for the adjustments: $S_{2}$. white-light senon source uned for optical multiplication by $4\left(\mathrm{R}_{1}\right.$ and $\mathrm{R}_{3}$ in their upper positions, $R_{2}$ in its lower position) ant by 12 ( $R_{1}$ in its lower position. $R_{2}$ and $R_{3}$ in upper position. A eclipsed): the movable mirron $.1 \mathrm{~m}$ is then alined with mieroscope $\mathrm{W}_{1}$ or $\mathrm{V}_{24}$, and the line T, engraved on the lower edge of the miror, can be pointed: If, fixed mirror: $O, O_{1}, O_{2} . O^{\circ}$. ohjectives: L. olservation telescope: R. orientahle mirmr: IM, splitting and compensating plates of the Viehelson interferometr. 
measurable by this procedure to about 100 millimetres. Furthermore, the Fizeau fringes are slightly asymmetric.

The new instrument uses a Michelson interferometer. These fringes are also formed between two mirrors, but one of these is virtual; it is the image of a fixed mirror. It can therefore be placed at the midpoint of the gage (fig. 11 (b)). The two sets of fringes have equal contrast and the length of gages which can be measured is doubled (it is about $200 \mathrm{~mm}$ with a natural cadmium source and $250 \mathrm{~mm}$ with a mercury-198 source). In addition, the fringes are symmetric. In short, the arrangement is the same as for the measurement of gages with the interferometric photoelectric comparator by the method of excess fractions (see p. 81). The temperature is determined in the same way; the refractive index of the air is measured with a built-in refractometer, or calculated from ambient conditions. On the other hand, the determination of the fractional parts of the interference order is made in this case by visual observation (fig. 11 (c)). The details of construction make the use of this instrument simple and fast. Thus, for example, a rotating carriage can hold up to nine gages, each wrung onto the base plane. The desired gage can be brought to the measuring position by external control.

The uncertainty of the measurement is generally 0.01 to $0.04 \mu \mathrm{m}$, depending on the length of the gage.

\section{Interferometric Measurement of the Geodetic Baseline}

This installation (fig. 12), completed in 1964, concerns only the interferometric measurement of the distance between the axes of the microscopes $M_{0}$ and $M_{24}, 24$ metres apart. The passage from this distance to that which separates the corresponding references, and the comparison of geodetic wires with the baseline established by these references, remain the same as described earlier (see p. 72 ).

Counting fringes would without doubt be applicable as a method, but there would be serious difficulties due to the consickerable number of fringes to be counted in a reasonable time (more than 75000000 with visible radiation), and to the necessity of realizing a high-quality motion of translation. An original method has been developed [13]: it is based on the principle of optical multiplication and uses Fabry-Perot etalons.

Monochromatic radiation which enters a Fabry-Perot etalon along its axis gives rise to narrow circular fringes observable with a telescope. By measurement of the angular diameters of a few of the rings for several radiations of known wavelength, the distance between the two mirrors of the etalon can be determined by the method of excess fractions.

In this way, the exact length $l_{0}$ of a 0.25 -metre etalon can be determined using several radiations of krypton 86, inchuding the standard radia- 
tion. Naturatly, the refractive index of air must be known, but this can be calculated from ambient conditions.

Now let there be a second etalon whose length $l_{1}$ is close to a multiple of the length of the first one, for example, a 1-metrc ctalon. By ilhuminating the system of these two etalons in line with an intense source of white light, one can observe interfcrence patterns. These can be simply explained by saying that a beam which makes a round trip in the $1-m$ etalon travels about the same distance as the beam which makes four round trips in the $0.25-\mathrm{m}$ etalon. These two beams give an interference pattern whose appearance depends only slightly on the wavelength and remains visible with a light as complex as white light. Many other beams of appreciable intensity are also present. Some of these produce interference, others do not but contribute to a lessening of the fringe contrast which is therefore decreased when the length ratio of the two etalons is increased. A method based on the displacement of the interference fringes when the orientation of one etalon is changed slightly permits determination of the small difference $l_{1}-4 l_{0}$. The length $l_{1}$ is thus obtained.

Quite analogous phenomena are produced by observing, through a 1metre etalon, the light from a Michelson interferometer whose path difference of the two arms is a multiple of $l_{1}$. In particular one can obtain rigorous multiplication by 12 for 2 positions of the movable mirror of the Michelson interferometer: in one, the variable arm is shorter than the fixed arm by $12 l_{1}$; in the other, it is larger by $12 l_{1}$. To go from one of these positions to the other requires a $24 l_{1}$ displacement of the movable mirror. The installation is such that one of these positions is plumb with the microscope $\mathrm{M}_{0}$, and the other with $\mathrm{M}_{24}$. By pointing each microscope on a line engraved on the edge of the mirror, the distance between their axes is obtained.

The method described above is also suitable for measuring the distance between any two of the microscopes located between $M_{0}$ and $M_{24}$, since this distance is always a multiple of $1 \mathrm{~m}$.

Putting this into operation allowed appreciable progress, notably for

the measurement of geodetic tapes observed directly with the microscopes; in fact the determination of the 24-metre distance between microscopes $M_{0}$ and $M_{24}$ is now carried out to an estimated 2 or $3 \mu \mathrm{m}$.

\section{Complementary Studies of the Standard Radiation}

The accuracy of interferometric length measurements is most often limited by instrumental imperfections: defects of mirror flatness, expansion, drift, etc.These limitations correspond to imperfect utilization of the possibilities offered by monochromatic radiation. Errors due to instrumental defects have been studied at the BIPM since its creation. We most often know how to eliminate their effects or how to allow for them by appropriate corrections. 
With the 1960 definition of the metre, it became essential to study the limitations due to the radiation itself: a krypton lamp always gives an imperfect realization of the length unit.

\section{Extrapolation to the Unperturbed Atom}

The definition of the metre refers to an atom of krypton 86 which is not perturbed, therefore isolated and stationary. In a spectral lamp, one must take account of several effects which influence the emitted radiation: effect of pressure due to interactions between atoms, Doppler effect due to thermal agitation of atoms or their entrainment by the exciting electric current.Thermal agitation betrays itself by a practically symmetrical broadening of the spectral profile, without displacement of the mean wavelength. On the other hand, one must extrapolate to zero pressure and current to evaluate the effects of pressure and current on the wavenumber ${ }^{6}$ of the emitted radiation (fig. 13). When the krypton-86 lamp is used under the recommended conditions ( $p .76$ ), these effects compensate each other and the wavelength of the emitted radiation (fig. 13) corresponds to that of the definition of the metre to better than 1 in $10^{8}$.

\section{Spectral Profile}

The broadening of the spectral profile is mainly due to the Doppler ef fect from thermal agitation. When the lamp is used under the recommended conditions, the half-width of the spectral profile, in terms of wavenumber, is around $\delta \sigma=1.3 \mathrm{~m}^{-1}$ (fig. 14). This broadening limits the visibility of the interference fringes for large path differences. In a Michelson interferometer, the visibility of the fringes of the standard radiation is no more than about 0.01 at a path difference of $D=0.9 \mathrm{~m}$ (fig. 15); it is still less for the other usual radiations (lasers excepted). It is thus practically impossible to measure directly lengths greater than $1 \mathrm{~m}$ with the standard radiation itself.

A more complete study [16], in which account must be taken of intensity fluctuations, shows that the relative precision of interferometric measurements reaches a maximum of the order of $10^{9}$ for lengths of the order of 0.4 metre (fig. 16).

We have also been able to demonstrate a very small asymmetry of the spectral profile [15] whose origin is still unexplained. In interferometry, this asymmetry betrays itself, in principle, by a relative variation of the

\footnotetext{
${ }^{6}$ To describe the spectral properties of a radiation, it is preferable to use the wavenumber $\sigma$ or the frequency $f$ which are related to the wavelength $\lambda$ and the speed of light in vacuum by the relations: $\sigma=1 / \lambda=f / c$.
} 


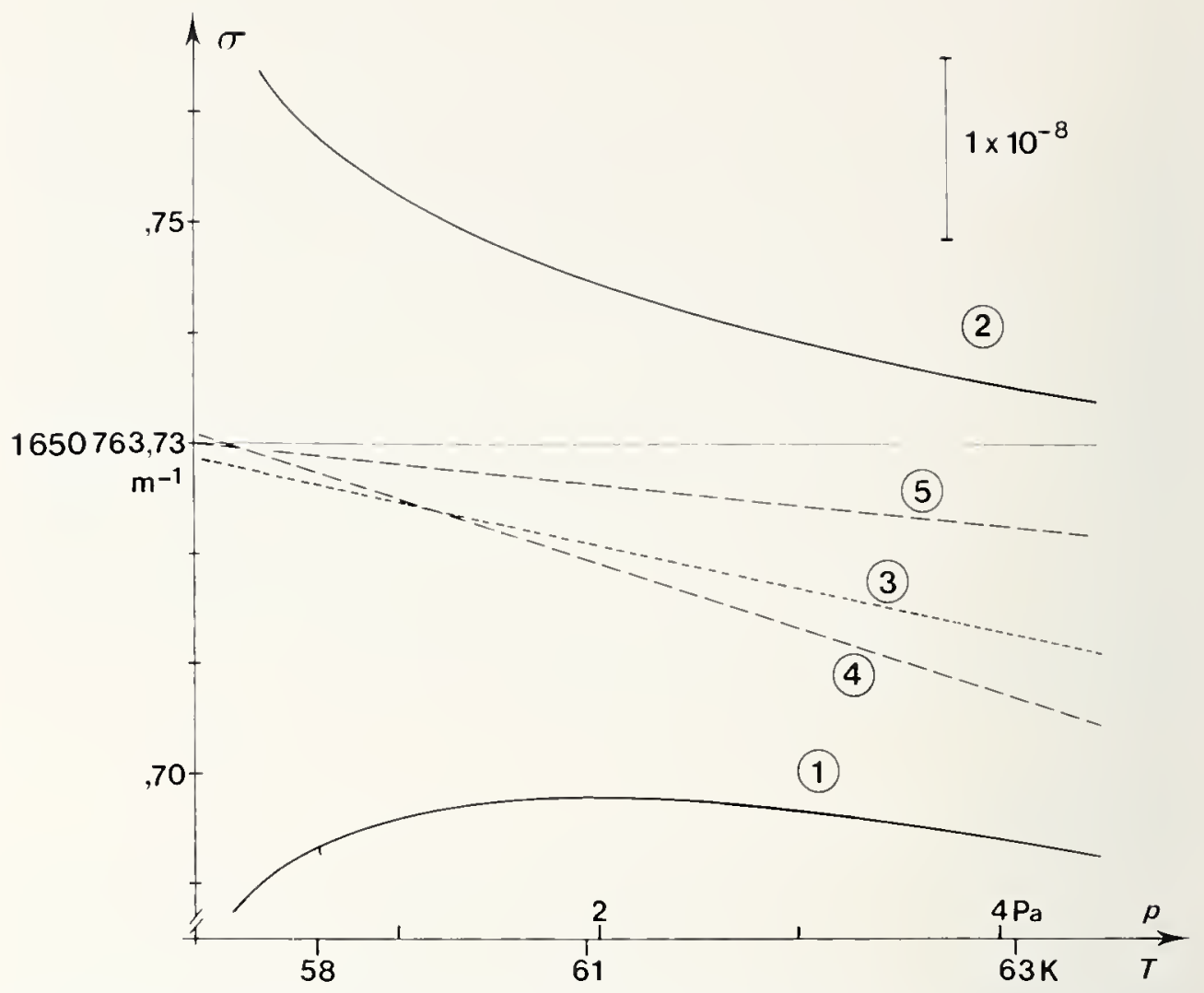

Figure 13. Extrapolation to zero pressure in a krypton-86 lamp [14]. The pressure $p$ in the lamp is the saturated vapor pressure of solid krypton at temperature $T$ : it is controlled by changing $T$. The current density is held at $0.33 \mathrm{~A} / \mathrm{cm}^{2}$. The wavenumber $\sigma$ of the emitted radiation depends on the pressure and on the direction of observation. Curve (1): observer at the cathode end (light emitted in the current direction). Curve (2): observer at the anode end (light emitted in the direction opposite to the current).

By taking the averaye (straight line (3)). one eliminates the Doppler effect responsible for the difference between curves (1) and (2). The value of the wavenumber extrapolated to zero pressure corresponds, to better than $l \times 10^{-x}$. to that obtained at $T=63.15 \mathrm{~K}$ (triple point of nitrogen) at the anode end (curve (2)).

Under different conditions, one oltains lines like (4) (current density, $0.78 \mathrm{~A} / \mathrm{cm}^{2}$ ) or (5) (transverse observation) which allow placing the value $1650763.73 \mathrm{~m}^{-1}$ on the $\sigma$ scale. 


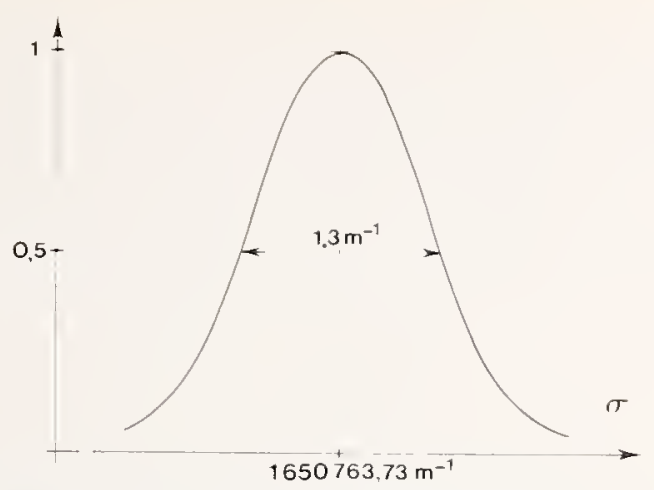

Figure 14. Spectral profile of the standard radiation [15]. The standard radiation is emitted by a krypton-86 lamp operating under recommended conditions. The spectral profile is deduced from the variation in the visibility of interference as a function of path difference in a Michelson interferometer. The lack of symmetry is imperceptible. The ordinate at the peak is taken as unity.

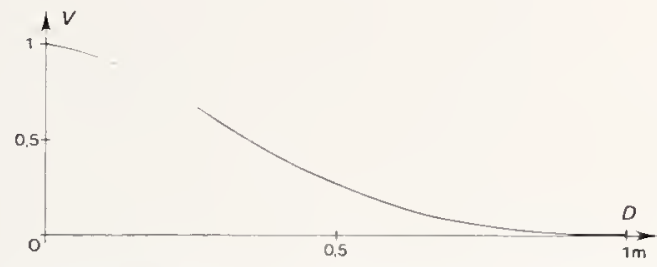

Figure 15. Variation of the visibility $V$ of interference as a function of path difference $D$ in a symmetrical Michelson interferometer, for the standard ratiation.

$V$ is defined (Michelson) in terms of the maximum $I_{M}$ and minimum $I_{m}$ intensities of the fringes, by

$$
V=\left(I_{\mathrm{M}}-I_{\mathrm{m}}\right) /\left(I_{\mathrm{M}}+I_{\mathrm{m}}\right)
$$

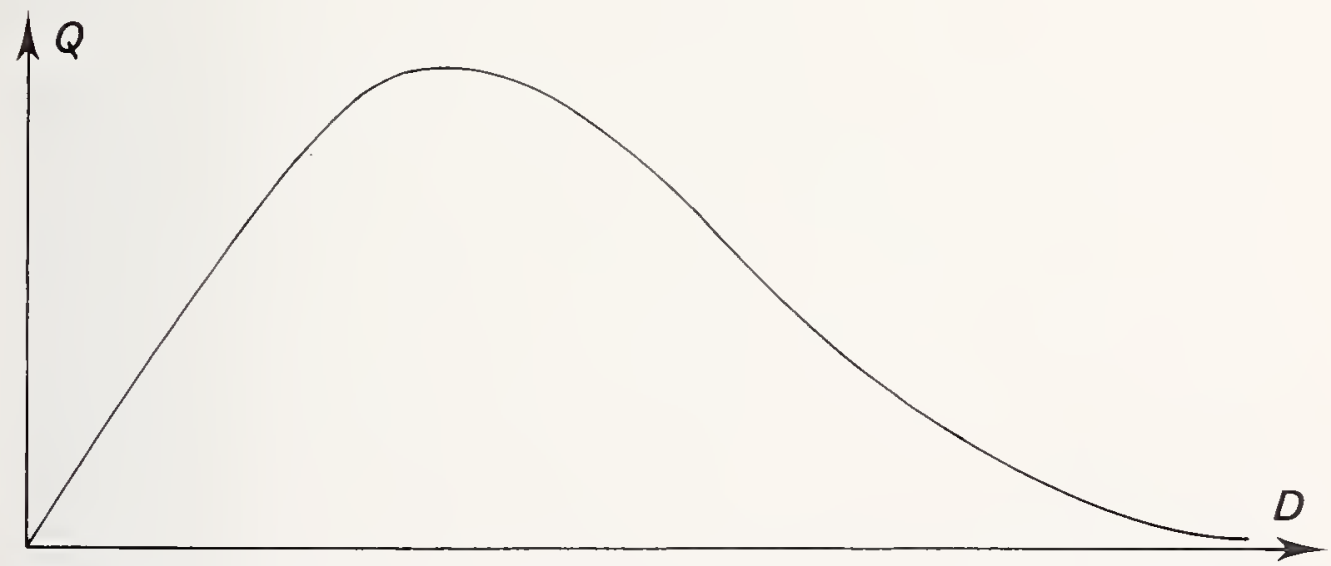

Figure 16. Variation of the relative precision of measurement as a function of path difference $D$. In measuring a length $l$ (or a variation of interference order $\Delta_{p}=\Delta D / \lambda$ ) it is always desirable to use the path difference variation $\Delta D=l$ between $D_{1}=-l / 2$ and $D_{2}=+l / 2$. In this case the precision is characterized by $Q=l / \delta l, \delta l$ being the uncertainty in $l$. The scales are arbitrary. For small path differences the interference visibility varies little and $Q$ increases with $D$; for large path differences. the reduction of visibility becomes the dominant effect. 
order of $1 \times 10^{-8}$ in the apparent wavelength as a function of path difference: it is fortunately without practical consequence: when the maximum precision is used, the apparent wavelength corresponds closely to the average of the wavelengths at the peak and center of gravity of the spectral profile [17] for whatever type of interferometer used.

\section{Reproducibility}

To evaluate the reproducibility of the realization of the length standard, it is sufficient to compare the wavelengths of the radiations emitted by various krypton-86 lamps. The lack of reproducibility is thus evaluated to be $\pm 2 \times 10^{-9}$ [18]. A better check consists in comparing length measurements in different laboratories, made on an "object" whose stability and reproducibility are certainly better than those of the length standard it self. The measurement of wavelengths of stabilized lasers (see p. 98) has recently permitted such comparisons. The dispersion of the measurements is less than $\pm 4 \times 10^{-9}$. As this last value includes the inevitable imperfections of utilization, it well confirms the preceding evaluation. It appears impossible to reduce the uncertainty of measurements using the krypton lamp to less than $1 \times 10^{-9}$.

\section{Lasers}

Since 1955, the invention of the $\operatorname{laser}^{7}$ has brought spectacular progress to interferometric procedures. The BIPM has been particularly interested in continuously operating helium-neon lasers (fig. 17): only these are involved in the following discussions. These laser sources are very directional, very intense, and can be very monochromatic. It is especially this last property which interests us here: the spectral width $\delta \sigma$ of the radiation from a laser can be less than $0.001 \mathrm{~m}^{-1}(\delta f=30 \mathrm{kHz})$, which al. lows observation of interferences of perfect contrast at all customary path differences and up to several kilometres.

Unfortunately, although the instantaneous spectral profile is very narrow, the mean wavelength $\lambda$ varies rapidly with time. The mean wavelength must be inside the relatively broad spectral band (spectral width of the order of $1 \mathrm{CHz}$, or $\Delta \sigma=3 \mathrm{~m}^{-1}$ ) in which laser action can occur in the mixture of helium and neon suitably excited by an electrical discharge. In this band. the laser oscillations fix themselves spontaneously to one or several

\footnotetext{
'The term laser. which has been accepted into numerous languages. is a contraction of "light amplification by stimulated emission of radiation." Originally the term "laser effect" was applied to the phenomenon of amplification of visible waves: usage now attributes the name "laser" to hight sources using the laser effect.
} 


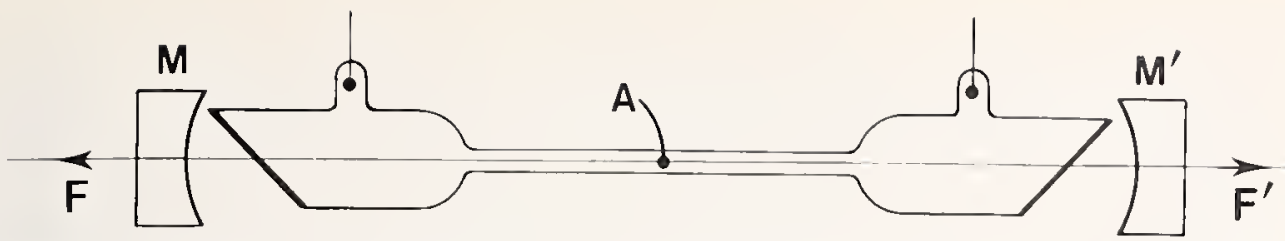

Figure 17. Sketch of the principle of a helium-neon laser $(\lambda \approx 0.633 \mu \mathrm{m})$. M, M'. slightly transparent concave mirrors. forming the resonant cavity; their curvature keeps the light beam in the cavity throughout numerous round trips. A. mixture of helium (about $85 \%$ ) and neon (about $15 \%$ ) under a total pressure of $300 \mathrm{~Pa}$, cxeited by an electrical discharge; this medium amplifies the waves of $\lambda \approx 0.633 \mu \mathrm{m}$ which traverse it; the oblique windows (Brewster angle) have a negligible reflection factor for light polarized in the plane of the figure. F, F', emerging beams, polarized in the planc of the figure.

frequencies corresponding to resonances of the cavity: for a cavity of spectral length $L$, the resonance frequencies $f$ (for oscillations of axial symmetry) correspond to $L=k \lambda / 2$, whence $f=k c / 2 L$, where $k$ is an integer. Different values of $k$ correspond to different axial modes of oscillation of the laser. For a sufficiently short cavity, only a single oscillation mode can be excited and the laser is then said to be unimodal; this is the only case with which we are concerned. The frequency $f$ remains dependent on the optical length of the cavity. Since the cavity contains the optical medium, electrically excited, the stability of $L$, hence also that of $f$, is always mediocre.

The metrological interest in lasers increased many fold when it was learned how to stabilize the frequency (or the wavelength) of the emitted radiation by various artifices.

\section{Stabilized Lasers}

The power radiated by a laser varies as a function of the optical length of the cavity, hence as a function of the frequency $f$. For each mode, it has a maximum on which is superposed a slight depression, the Lamb dip, under certain excitation conditions (see fig. 19 (a) and p. 97). The maximum (or the superposed minimum) is located at a claracteristic frequency of the amplifying medium. This property is easily used to stabilize the length of the laser cavity through feedback, for example by the procedure indicated in figure 19 (a) and (d), in such a way that it corresponds to the maximum (or minimum) of power. The related variations of wavelength of a laser stabilized in this way can be of the order of $1 \times 10^{-8}$ for periods of the order of several months. Over a longer time, a drift (fig. 18) is usually observed, apparently due to diffusion of helium through the walls. Several lasers of this type are used at the BIPM, for example for length measurement by fringe counting; their wavelengths must be calibrated regularly.

The length of the laser can also be slaved in such a way that the emitted wavelength corresponds to a resonance of an auxiliary optical cavi- 
ty [19]. The optical length of the auxiliary cavity then determines the wavelength of the laser and the stability of one controls the stability of the other. Here again, calibration is indispensable and it must be repeated periodically.

A much more effective procedure which we shall discuss in more detail consists in forcing coincidence between the frequency of the laser and that of a "saturated absorption line."

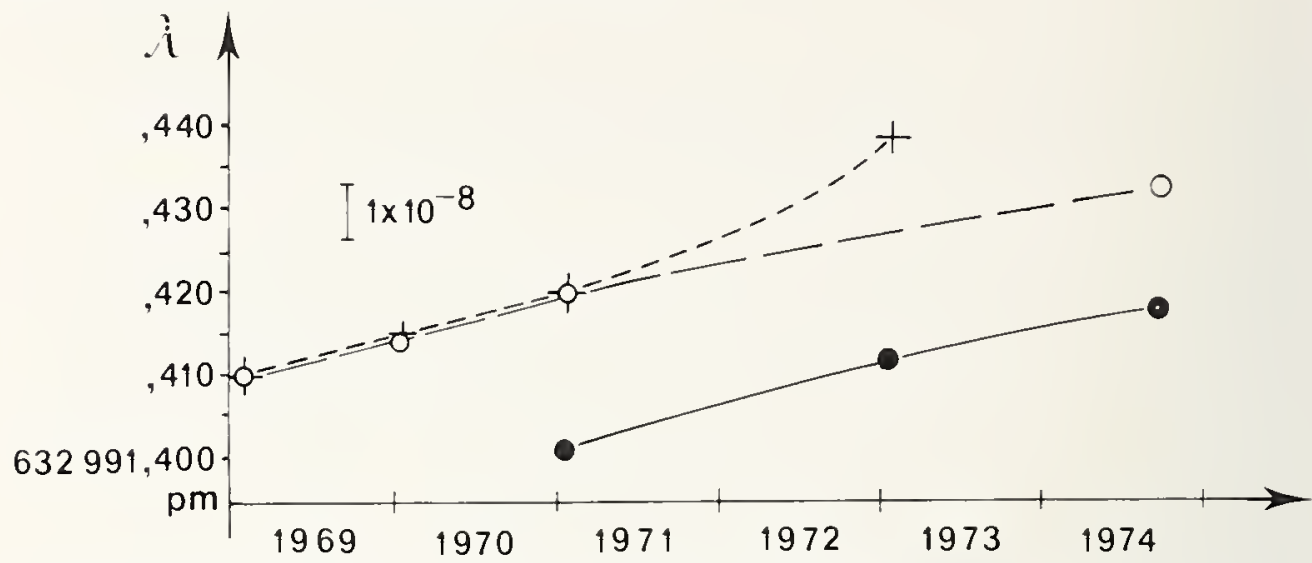

Ficure 18. Change with time of the wavelength of three helium-neon lasers locked 10 the Lamb dip of the emission. The wavelength in riven in picometres $\left(1 \mathrm{pm}=10^{-12} \mathrm{~m}\right)$.

\section{Lasers Locked to a Saturated Absorption Line}

An absorption cell cont aining. under low pressure, of the order of $1 \mathrm{~Pa}$. a gas which exhibits an absorption line within the Doppler width of the laser frequency, is placed in the resonant cavity: for example, a methane cell for a helium-neon laser operating at $3.39 \mu \mathrm{m}$. The absorption reduces the power emitted by the laser (fig. 19 (b)).

The gas molecules, at rest, can absorb radiation of frequency $f_{0}$. If the laser oscillates at a frequency $f$ near $f_{0}$. the waves which propagate along the cavity can nevertheless be absorbed by certain molecules of the gas. For this to happen. it is sufficient that the velocity of these molecules, due to thermal agitation. have a longitudinal component such that. by Doppler effect, they "see" a wave of frequency $f_{0}$. If. for example. $f$ is less than $f_{0}$. the waves which propagate to the right are absorbed by molecules travelling to the left and vice versa: if $f$ is higher than $f_{0}$. the phenomenon is similar under interchange of the roles of the two families of waves. If $f=f_{0}$. the two families of waves are absorbed by a single class of molecules: those whose velocity is perpendicular to the axis of the laser and for which the Doppler effect vanishes.

When the absorbed radiation is very intense. there is also a phenomenon of saturation. Molecules which absorb radiation of frequency $f$ remain in an excited state for a certain time. The number of molecules 
(a)

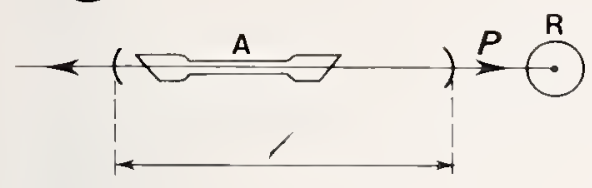

(b)

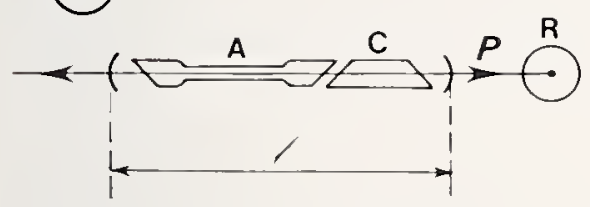

(c)

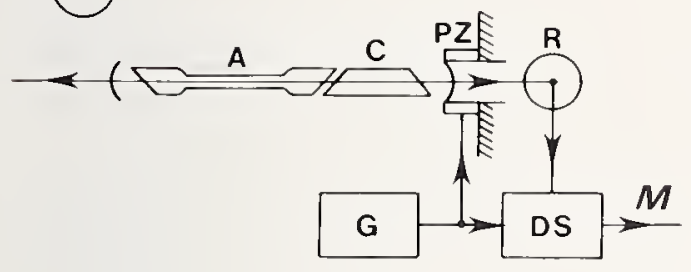

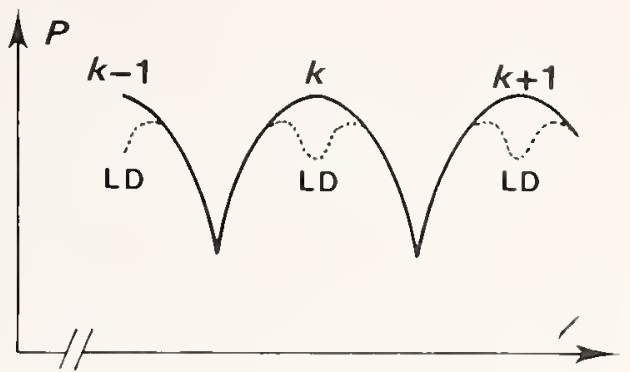

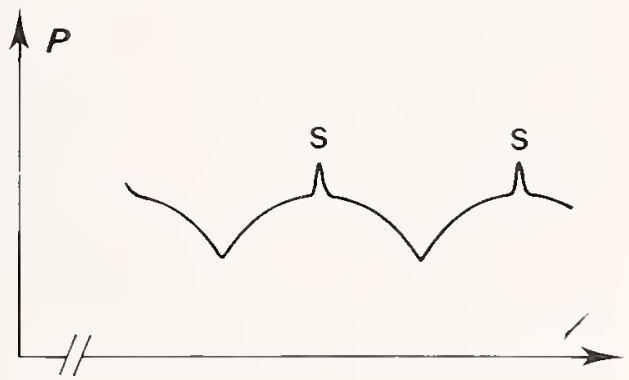

$\uparrow M$

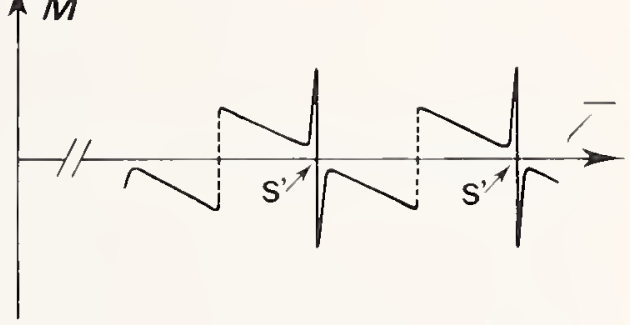

(d)

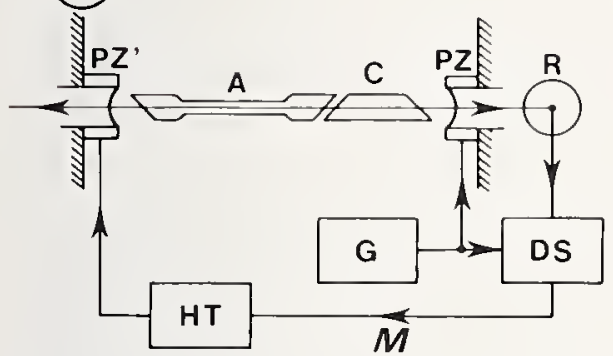

Figure 19. Principle of hoching a laser $(\lambda \approx 3.39 \mu \mathrm{m})$. (a) Variation of emitted power $P$ as a function of cavity length $l$. A, helium-neon amplificr tube. R, photoelectric sonsor. $k-1, k$. $k+1$, successive modes. Under eertain conditions of excitatinn, the Lamb dip LD is ob. servell. (b) The same laser with an ahsorption cell C containing methane under a pressure of 1 Pa. The emitted power is less. A peak $S$ due to saturated absorption is observed. (c) The cavity length $/$ is modulated with a very small amplitude at the frequency $F_{6}$; hy use of the generator $\mathrm{C}$ and the piezoelectric ceramic $\mathrm{PZ}$. The photoclectric current is modulated at the frequency $F_{1}$, witl an amplitude $m$ proportional to the first derivative of $P(l)$. The component at frequency $F_{1}$ is isolated and rectified by a synchromms-detector amplifier DS: the output voltage $M /$ of DS is proportional $10 \mathrm{~m}$. If the mean value $l$ of the length $l$ is varied. $M$ vanishes at $S$ which corresponds to the top of the peak $S$. and varies very rapidly in its neighlborhood. (d) $M$ controls a second ceramic PZ with the help of the amplifier HT. If $m>0, P Z$ lengthens the eavity: if $m<0$, it shortens it. The mean length stabilizes at one of the values for which $M$ vanishes (points $S$ '). corresponding (1) the top of one of the peaks S. 
remaining in the normal state diminishes, and with this, the capacity of the gas to absorb at frequency $f$ : there is saturation of the absorption.

In the laser cavity, this saturation occurs more readily for molecules which can equally absorb waves which propagate to the right or to the left. The absorption then decreases when the frequency $f$ of the radiation (a function of the cavity length) passes through the value $f_{0}$, and the radiated power then presents a "saturated-absorption peak." The molecules responsible for this peak being just those for which the Doppler effect vanishes, this peak is not broadened by the Doppler effect. ${ }^{8}$ One thus obtains peaks of width $\delta f$ less than $100 \mathrm{kHz}$ (relative width $1 \times 10^{-9}$ ) with methane at $\lambda \approx 3.39 \mu \mathrm{m}$ and less than $3 \mathrm{MHz}$ (relative width $6 \times 10^{-9}$ ) with iodine at $\lambda \approx 0.633 \mu \mathrm{m}$.

It is relatively easy to slave the cavity length by using the peak due to saturated absorption as a reference (fig. 19 (c) and (d)). Since this peak is very sharp, the slaving can be extremely precise.

Since the absorption is due to a nonexcited gas under low pressure, the frequency $f_{0}$ is subject to only a little perturbation. In addition, we can choose a gas whose useful absorption line is to a certain degree insensitive to electric and magnetic fields. This is the case for methane at $\lambda \approx 3.39$ $\mu \mathrm{m}$. There exist, however, some effects which broaden or displace the absorption line: duration of interaction with the waves limited by the transit time of the molecules, perturbation of the molecules by the intense electromagnetic field of the laser radiation or by nearby molecules, differences of saturation for different components when the absorption line exhibits a nonresolved structure, Doppler effect of the second order, etc. [18,20]. These effects are always small; the corresponding frequency displacements, which rarely exceed several kilohertz, say a few parts in $10^{10}$ for relative value, can in large measure be made reproducible.

Other parasitic phenomena can involve an apparent displacement of the peak due to saturated absorption. If this peak is located on the powerlength characteristic in a region of appreciable slope, its summit is displaced in relation to the frequency $f_{0}$ (fig. 20). Similarly, if parasitic electric potentials appear in the servomechanism, the length of the cavity may be stabilized to a value slightly altered from that which corresponds to the top of the peak.

These last effects are, however, weaker for a narrower peak. In this regard, saturated absorption lines provide an essential safeguard for metrology.

\footnotetext{
${ }^{8}$ There is an analogous phenomenon, predicted by W. E. Lamb, Jr., which causes the Lamb dip in the case of emission (fig. 19 (a)); consequently, the peak due to saturated absorption is sometimes called "inverse Lamb Dip." Since emission occurs in a strongly perturbed medium, the Lamb dip of emission is very broad and less stable than the peak due to saturated ab. sorption.
} 


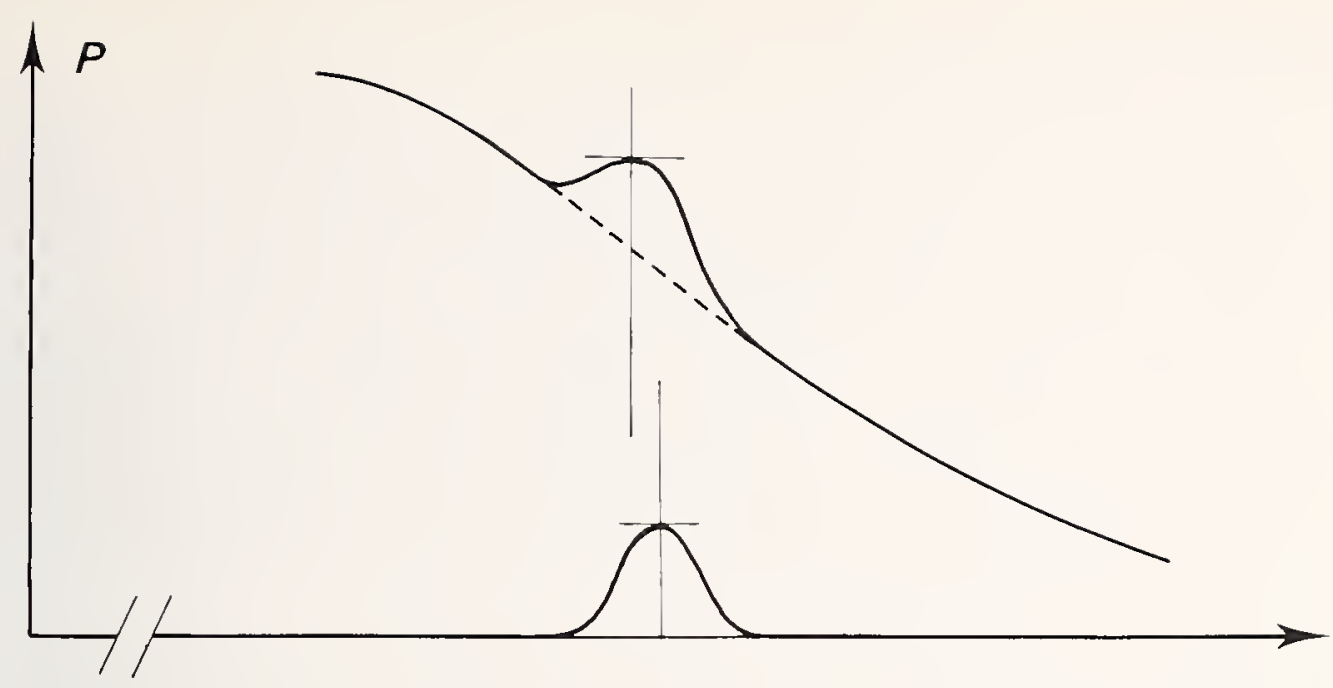

Figure 20. Shift of the top when a peak due to saturated absorption is superposed on the characteristic $P(l)$ in a region of appreciable slope.

\section{Measurement of Frequency Differences by Beats}

Thanks to the very high stability and to the very low spectral width of radiations from stabilized lasers, one can display and measure, as in acoustics, the beats between the radiations of two lasers having neighboring frequencies $f_{1}$ and $f_{2}$. To do this, a single photoelectric sensor is simultaneously illuminated by the beams from the two lasers, beams which are superposed with the help of a semi-transparent mirror. The illumination of the sensor and the current it delivers are modulated at the beat frequency $\Delta f=f_{1}-f_{2}$. The frequency $\Delta f$ of the current modulation is measured by the customary techniques of electronics.

This method is limited at high frequencies only by the response of the photoelectric sensor to the modulation of the light. It permits precise studies of the frequency displacement effects mentioned in the preceding section. It also permits intercomparison of frequencies, hence also of wavelengths, of lasers locked to the Lamb dip or to nearby saturated absorption lines. In this way, the intervals among a hundred absorption lines of iodine, in the neighborhood of $0.633 \mu \mathrm{m}$, have been measured. These intervals range from some 10 megahertz to a gigahertz [18]. This method is also used in the periodic calibration of lasers locked to the Lamb dip $(\lambda \approx$ $0.633 \mu \mathrm{m})$ by using a reference laser locked to the saturated absorption of iodine.

Finally, this is the only present method which permits evaluating the stability and reproducibility of the frequency (thus of the wavelength) of radiations from lasers locked to saturated absorption lines of iodine or methane. In this way have been measured, over times from several seconds to several hours, stabilities better than $10^{11}$ for iodine and $10^{13}$ for methane, with reproducibilites of the order of $10^{11}$ and $10^{11}$ respectively. 
Wavelengths of helium-neon lasers locked to methane $(\lambda \approx 3.39 \mu \mathrm{m})$ and to iodine $(\lambda \approx 0.633 \mu \mathrm{m})$ have been determined in several laboratories.

At the BIPM, we have used the Michelson interferometer for direct comparison of these wavelengths with that from the krypton-86 lamp [21]. If the interferometer is illuminated with two radiations of respective wavelengths $\lambda_{1}$ and $\lambda_{2}$ successively, and if the path difference is varied by $\Delta D$. the ratio of the variations of interference order, $\Delta p_{1}=\Delta D / \lambda_{1}$ and $\Delta p_{2}=$ $\Delta D / \lambda_{2}$, gives the ratio of wavelengths immediately. We have systematically used path differences $D$ of $\pm 375 \mathrm{~mm}$ leading to the maximum precision in the case of krypton radiation.

Figure 21 summarizes the results of these measurements. They have supplied the mean values of wavelengths which have been recommended by the CIPM [18]. They have also allowed experimental evaluation of the uncertainty with which the 1960 definition of the metre can be realized; this uncertainty does not exceed $\pm 4 \times 10^{-9}$.

In the case of iodine, several absorption lines can be used for locking. Their frequency differences, measured by the beat method, are known with a precision largely sufficient for deducing all the wavelengths from any one of them.

The frequency of the laser locked to methane, measured elsewhere [22], is $f=88376181627 \mathrm{kHz}$ to within $\pm 50 \mathrm{kHz}$. This measurement in conjunction with those of the wavelength $\lambda$ of the same laser, supplied the value of the speed of light in vacuo which was recommended by the CCDM in 1973 [18]:

$$
c=\lambda f=299792458 \mathrm{~m} / \mathrm{s} .
$$

The major part of the uncertainty comes from the uncertainty in the realization of the definition of the metre $\left( \pm 4 \times 10^{-9}\right)$, leading to an uncertainty in the value of $c$ of $\pm 1.2 \mathrm{~m} / \mathrm{s}$, nearly a hundred times smaller than that of the best earlier determinations.

It can be predicted that the speed of light and lock-stabilized lasers will play an important role in length measurements and will perhaps be the basis of a new definition of the metre. Studies carried out by the BIPM in this field now prepare for this possibility. 

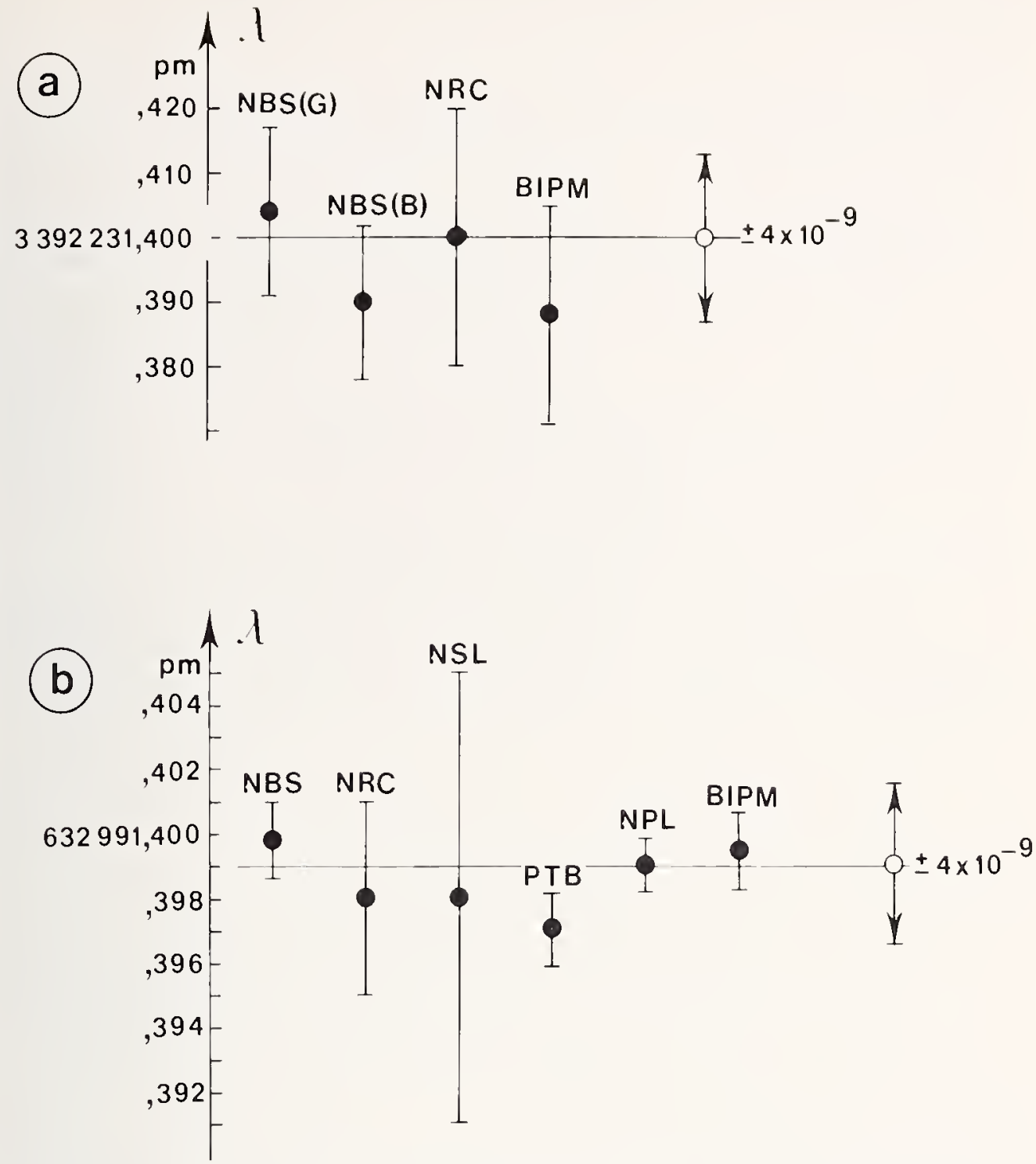

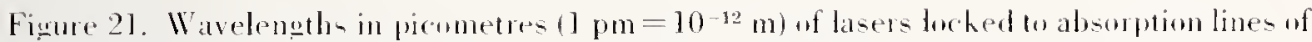
methan' (a) on indine (h). Values measured in various laboratories, will their uncertaintios, and recommended values. 


\section{Bibliography}

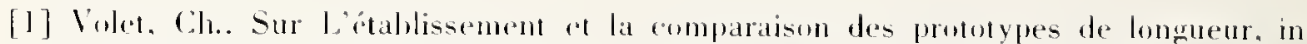

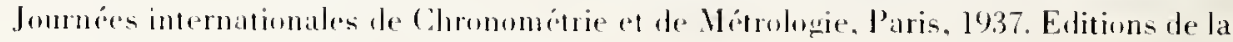
Revue d'Optipue. I'aris. pr. 6.3-70(19.39).

l'erard. A., Mandand, I.., and Volet, Ch.. Premiere verification périodigue des Mitres prototypes. Trawax ef Vemoires B.I.I'.H. 20, 86 pages (1944).

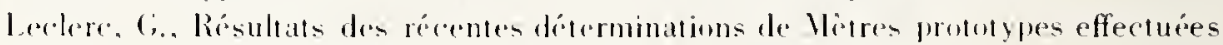

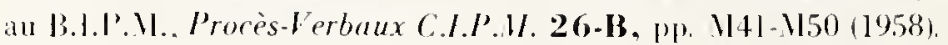

[2] Volet. Ch.. Sur la comparaison des ifalons de longueur i traits. Rev. Optique 21 , pp. $168-175(1942)$.

Ieclere. (;., lo nouveau comparateur du Bureau International pour les Métres protutypes. Procès-I'erbaux C.I.P.H. 25, pl. 146-153 (1956).

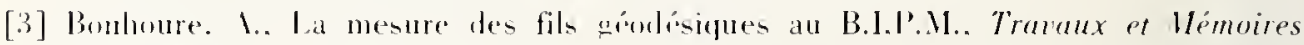
B.I.P..H. 22(2),61 pages (1966).

[4] Viehelson. 1. 1.. Détermination expoimentale de la valeur du mintre en longueurs loumles lumineuses. Travaux ef Wemoires B.I.I'.W. 1 1,237 payes (1895).

Benoit. J. R., Fabry, C.h.. and P'erut. A. Nouvelle determination du rapport des longueurs l'onde fondamentales avec l'unité métrique. Travaux el Mémoires B.I.P.M. 15. 134 paleses (1913).

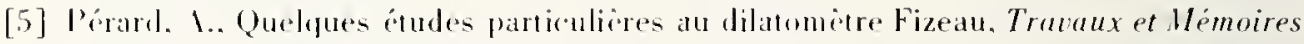
B.I.P..H. 19, 127 pages (1934).

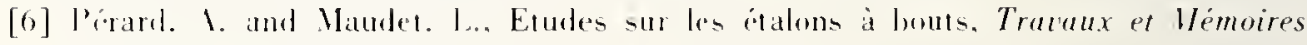
B.I.P..H. 17,91 pageso(1927).

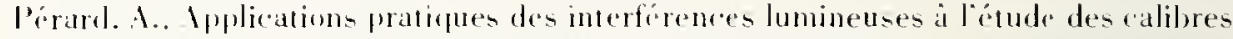
industriels. Travaux et Mémoires B.I.I..H. 18,85 pages (1930).

[7] Proces-Iorbaux C.I.I..1K. 12, p. 21 (1927). and 21,p. 25(1948).

[8] Terrien. J. and Hamon. J. Sur la mesure interfécentielle des longueurs par une méthode dohservation photólectrique. C.R. Acad. Sri. 243, pp. 740-742 (1956).

Terrien, J., l'erlectionnements a l'emploi d'un ensemble phutumultiplicateur interférumitre de Michelson pour les mesures de longueur dionde el de profil spectral des ratiations optiques de grande linesse. Optica feta 6, No. 4. p). 301-307 (1959).

[1) C.C.D.W., 30 nesosion. p. $173(1962)$.

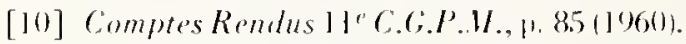

[11] Camr. l'.. Installation et utilisation du comparateur phonoflectrique et interfórentiel du 13.I.IP.M., Metrologia 2, No. 1, pr. ]3-23(1966).

[12] Ciacomo. P’. Iamon. J.. Hostache. J.. and Carre. P’. L tilisation du comptane de franges l'interfereneres putur des mesures de lonwueur de hante précoision, Metrologia 8, Nu. 2. lip. $72-82(1972)$.

[13] 'Terrien. J.. Irojet d'une mesure interlérentielle de la lase de 24 m du B.I.I’.M.. Ann. trul. Srient. Fennirae $41 / / 61,1) .263-268(1961)$.

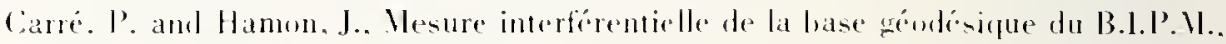
Metrologia 2. Vo. 4. pp. 143-150 (1966). 
[14] Terrien, J. and Hamon, J., Mesure de la longueur d'onde des radiations étalons secondaires et de la radiation étalon primaire sans perturbation, C.C.D.M., $3^{e}$ session. pp. 76 82 (1962).

[15] Rowley, W. R. C. and Hamon, J., Quelques mesures de dissymétrie de profils spectraux, Rev. Optique 42, pp. $519-531$ (1963).

[16] Terrien. J., Observations photoélectriques a l'interféromètre de Michelson, J. Phys. Radium 19, pp. 390-396 (1958).

[17] Giacomo, P., Dissymétrie du profil spectral et nombre d'ondes apparent de la radiation étalon du krypton en interférométrie, C.C.D.M., $5^{*}$ session, pp. M126-M132 (1973); see also Recueil de Travaux du B.I.P.M. 4, articles 9 and 10. 14 pages (1973-1974).

[18] C.C.D.M., $5^{e}$ session, pp. M15-M44 (1973).

[19] Tanaka. K. and Sakurai, T., Méthode pour stabiliser la fréquence d'un laser He-Ne non modulé, C.C.D.M., 4 e session, pp. M75-M76 (1970).

[20] Hall, J. L., Saturated absorption line shape, in fundamental and applied laser physics, Proc. of the Isfahan Symposium, 1971, M. S. Feld, A. Javan, and A. Kurnit, Eds., John Wiley \& Sons, New York, London, Sydney, Toronto, pp. 463-477 (1973).

[21] Giacomo, P., Longueur d'onde du laser asservi sur le méthane rapportée à la longueur d'onde étalon de la définition du mètre, in Atomic Masses and Fundamental Constants, Proc. of the Fourth International Conference, Teddington, 1971, J. H. Sanders and A. H. Wapstra, Eds., Plenum Press, London and New York, pp. 348-354 (1972).

[22] Evenson, K. M., Wells, J. S., Petersen, F. R., Danielson, B. L., and Day, C. W., Accurate frequencies of molecular transitions used in laser stabilization: the $3.39 \mu \mathrm{m}$ transition in $\mathrm{CH}_{4}$ and the $9.33 \mu \mathrm{m}$ and $10.18 \mu \mathrm{m}$ transitions in $\mathrm{CO}_{2}$, Appl. Phys. Letters 22, pp. 192-195 (1973). 



\section{CHAPTER IV}

\section{GRAVIMETRY}

\section{Metrological Importance of the Knowledge of the Acceleration of Gravity}

The most precise method of realizing a known force $F$ consists in utilizing the action of the terrestrial gravity field on a mass $m$, known by comparison with the standard kilogram. This action, when it exerts itself on a body free to move vertically, gives it an acceleration $g$. The fundamental relation of dynamics $F=m g$ permits relating $F$ to the base quantities length, mass, and time provided that the value of $g$ is itself determined in an absolute manner by means of measurements of length and time.

For example, the definition of the ampere and consequently of all the electric units involves a force and therefore in practice requires the knowledge of g. Similarly, the measurement of pressure with the aid of liquid manometers requires not only the measurement of the height of a column of liquid and the determination of the mass density but also the knowledge of $g$.

The importance of the knowledge of $g$, with the maximum precision, did not escape the CIPM; as early as 1886, it stated that the anomalies of gravity and the irregularities in the form of the Earth required more and more serious attention for barometric reductions; it recommended that the acceleration of gravity be determined locally, at places where fundamental barometric measurements are carried out and particularly at the Pavillon de Breteuil. Three methods were utilized successively at the BIPM; the first was based on the utilization of reversible pendulums; the second utilized the free fall of a graduated scale; the third, to which further improvements are still being made, employed the symmetric free motion (ascending, then descending) of an optical reflector; it is called the "method of two stations."

\section{Reversible Pendulums}

In 1888 , a measurement of the acceleration of gravity was carried out at the Pavillon de Breteuil by Defforges of the Geographic Service of the 
French Army, following a request from the CIPM [1]. The use of reversible pendulums of symmetric exterior form and having intcrchangeable knife edges permitted elimination of the influcnce of air resistance and of the imperfection of the knife edges. The use of pendulums of different lengths but of the same mass, and oscillating on the same knife edges, eliminated the combined effects of the support motion and the sliding of the knives as well as certain systcmatic errors in the determination of the knife edge separations of the two pendulums. The uncertainty of the result obtained $\left(g=9.80991 \mathrm{~m} / \mathrm{s}^{2}\right)$ seemed to be less than $5 \times 10^{-5} \mathrm{~m} / \mathrm{s}^{2}$, or 5 units in the last place.

The absolute measurement carried out at Potsdam in 1905 by $\mathrm{F}$. Kühnen and Ph. Furtwängler [2], also based on the use of reversible pendulums, served as the point of departure for the "Gravimetric system of Potsdam." From the value of $g$ obtained at this location, the values of $g$ at various points on the Earth have been deduced by use of connections, that is to say by measurements of differences, effected by means of relative gravimeters of either pendulum or spring type. In this system, the value of $g$ at the Pavillon de Breteuil (point A) is $9.809398 \mathrm{~m} / \mathrm{s}^{2}$. Note that the value of Defforges differs from this by $5 \times 10^{-4} \mathrm{~m} / \mathrm{s}^{2}$.

The exactness of these measurements, however remarkable for the time, was put in doubt and several new determinations were carried out by similar methods between 1920 and 1940; the best known are those by P. R. Heyl and G. S. Cook in the United States and by J. S. Clark in Great Britain. It appeared that the Potsdam system was blemished by an error of 10 to $20 \times 10^{-5} \mathrm{~m} / \mathrm{s}^{2}$. The difficulties of methods based on the use of pendulums seem to consist of the exact evaluations of certain corrections [3], notably those which are due to the elastic distortion of the oscillating pendulums. It was desirable to conceive an entirely different method.

\section{Free Fall of a Graduated Scale}

In 1946, timed motion pictures of a graduated scale in free fall in vacuo was proposed [4]. The law of motion, that is to say the expression for the vertical distance $h$ of the center of gravity of the scale from a horizontal reference plane, as a function of the time $t$ counted from an arbitrary origin, is $h=h_{0}+v_{0} t+1 / 2 g t^{2}$, assuming $g$ const ant throughout the trajectory of the scale. It is seen that knowledge of the position of the scale for three values of $t$ is theoretically sufficient for the computation of $g$. One can therefore be content with three images; actually, one uses many more in order to increase the precision. A preliminary determination by this method was made; it was finished in 1951. It appears that the result obtained $\left(9.80916 \mathrm{~m} / \mathrm{s}^{2}\right)$ suffered from several systematic errors, due mainly to friction of the residual air. 
The studies were continued with the aim of improving the accuracy of the measurements. These researches were completed in 1958 with a new determination [5]. Let us give some details of this work.

The body which undergoes free fall (fig. 1) is a 1-metre scale of platinum-iridium bearing millimetre divisions over its whole length. The scale is furnished with ferrules used on the one hand to keep it vertical between two supports, and on the other hand, after the fall, initiated by removal of the lower support, to catch the scale by a device giving gradual braking. The mean temperature of the scale, also the temperature gradient, is determined by the use of two thermocouples attached to the support.

The fall is carried out in a cylindrical enclosure within which is a vacuum of the order of $0.007 \mathrm{~Pa}$. A photoelectric device permits verifying that the scale remains vertical during its fall.

The graduation of the scale is photographed at regular intervals during the fall. For that purpose, an optical system forms, on a film placed on a rotating drum, the superposed images of the marked surface of the scale and of a fixed reference mark.

Illumination is provided by a spark gap controlled by a quartz oscillator; it produces flashes at regular intervals, adjusted as appropriate to the experiments, say at the values $10 \mathrm{~ms}$ or at $8 \mathrm{~ms}$.

The initiation of the fall and the start of the train of flashes are controlled by the rotation of the drum itself. Thus, for each fall, about 50 images are available on the film. After development, the exact positions of

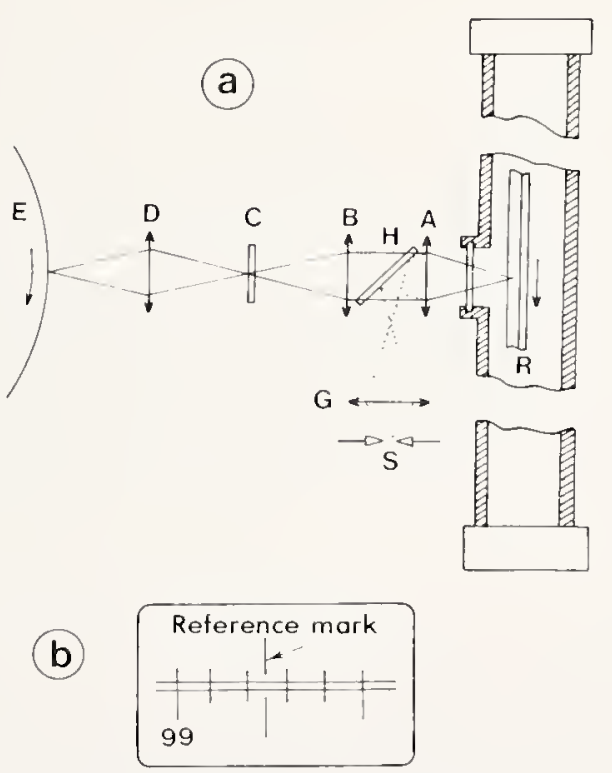

Figure 1. Measurement of g by method of fall of a graduated scale (a) Diagram of the optics. The gratuation of the scale $R$ is illuminated by sparks in the gap $S$. through the ohjectives $G$ and $A$ and the semi-transparent plate $H$. The uhjectives $A$ and $B$ form an image of this graduation on the wlass plate $C$ carrying a reference mark. The objective $D$ wives, on the film placed in the drum E. an image where the graduation and reference marks are superposed. (b) Appearance of an image of the marks of the scale and the reference. 
the scale are obtained by locating each image of the graduation of the scale and the fixed reference mark by means of a micrometer microscope. Corrections are applied to the positions found, to take account of the calibration of the subdivisions of the scale. Computation of the acceleration is made by the method of least squares making equal use of all the images. The value obtained is corrected for the exact length of the scale considering its temperature, also for the departure of the exact frequency of the quartz oscillator, determined by comparison with a standard frequency transmission, from its nominal frequency.

The value of $g$ cannot be considered as constant throughout the height of the fall, but we can assume that it varies linearly as a function of elevation, its increase being about $3 \times 10^{-6} \mathrm{~m} / \mathrm{s}^{2}$ when the elevation diminishes by $1 \mathrm{~m}$. It is established that the calculation method used gives the value of $g$ at a point situated at the distance $2 h_{\mathrm{T}} / 7$ below the location of the center of gravity before the drop, $h_{\mathrm{T}}$ being the total height of fall. The result obtained is transferred to reference point A of the BIPM gravimetric station by means of an experimental value of the gradient of $\mathrm{g}$.

Finally, the attraction of the Sun and Moon produce a variation of $g$, called gravitational tide, whose maximum amplitude is $1.6 \times 10^{-6} \mathrm{~m} / \mathrm{s}^{2}$. Tables resulting from a theoretical evaluation permit calculating the small correction which results from this effect.

Finally, the value found at the Pavillon de Breteuil (point A) is $g=$ $9.809280 \mathrm{~m} / \mathrm{s}^{2}$. We note that the value of $g$ at this point on the Potsdam system differs from this by $11.8 \times 10^{-5} \mathrm{~m} / \mathrm{s}^{2}$.

\section{Method of Two Stations}

This method, advocated as early as 1947 [6], was put into operation at the BIPM beginning in 1960 [7]. The first results were obtained in 1966. Their relative precision was then estimated at $1 \times 10^{-7}$. Improvements of the apparatus since that date have allowed reaching the present precision (early 1974) of $3 \times 10^{-9}$. To get a feel for this precision, one can note that $g$ varies by $3 \times 10^{-9}$ (in relative value) when the altitude of the point of measurement changes by $1 \mathrm{~cm}$.

\section{Principle}

A body projected vertically upward in vacuo crosses two horizontal planes, $S_{1}$ and $S_{2}$ (the "stations"), whose separation $h$ is known (fig. 2 ); if $t_{1}$ is the time between the two passages of the center of gravity of the body at the lower station in the course of its ascent and descent, and $t_{2}$ the analogous quantity for the upper station, it is easily established that $g$ is given by the relation $g=8 h /\left(t_{1}{ }^{2}-t_{2}{ }^{2}\right)$. 


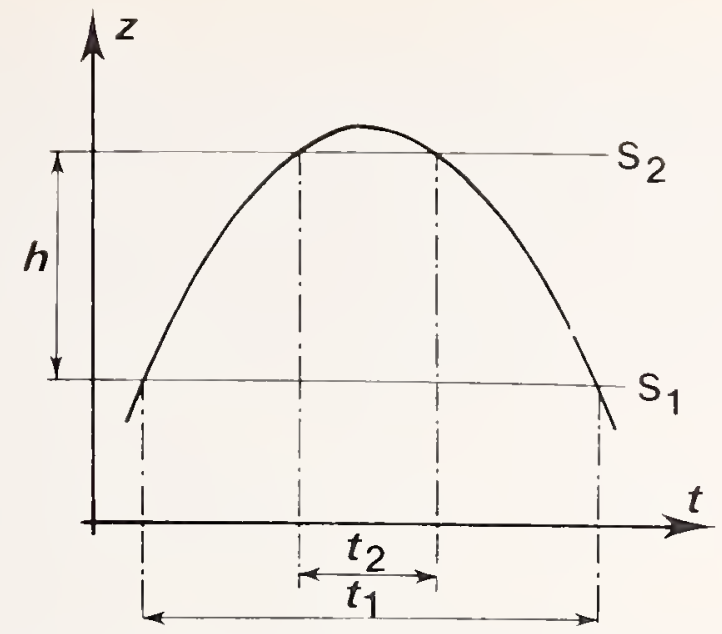

Figure 2. Principle of the measurement of $g$ by the method of two atations. The curve (parabola) represents. as a function of time t, the height z reached by the center of wravity of an object launched vertically upward. If $t_{1}$ and $t=$ are the intervals which separate the ascending and descending passages of the point past the station $S_{1}$ and $S_{2}$ of separation $h$. the acceleration of the object is

$$
g=8 h /\left(t_{1}^{2}-t_{2}^{2}\right)
$$

In fact, by virtue of the vertical gradient of $g$ which is supposed constant along the trajectory, this formula gives the exact value of $g$ at a point situated at the distance $h / 6+h^{\prime} / 3$ below the summit of the trajectory. $h^{\prime}$ being the distance between the upper station and the summit.

This method presents the advantage of automatically eliminating the systematic errors due to the possible braking forces due either to residual air or to eddy currents caused by the inhomogeneity of the Earth's magnetic field, since these forces change sign with the reversal of velocity of the body: they add to its weight during the ascent and subtract from the weight during the descent. Furthermore, this facilitates the time measurement since at the beginning and end of each of the intervals $t_{1}$ and $t_{2}$ the body passes the same position with the same velocity (apart from sign).

However, if high precision is sought, putting this method into operation presents serious metrological and technical difficulties. The relative precision of $3 \times 10^{-9}$ for $g$ requires the following precisions: $1 \mathrm{~nm}$ for $h$ of the order of $400 \mathrm{~mm}$. $0.3 \mathrm{~ns}$ for $t_{1}$ of about $0.6 \mathrm{~s}$ and $1 \mathrm{~ns}$ for $t_{2}$ in the neighborhood of $0.2 \mathrm{~s}$. The body must be launched upward by a motion of vertical translation with extremely severe tolerances as to its direction $(3 \times$ $\left.10^{-5} \mathrm{rad}\right)$ and as to its motion in rotation $\left(1 \times 10^{-2} \mathrm{rad} / \mathrm{s}\right)$. Furthermore, it is necessary to use a reference base stable to a few nanometres, protected from ground vibrations and shocks due to the launching. 


\section{Implementation}

The essential part of the absolute gravimeter (fig. 3) is a fool-proof Michelson interferometer [8]. An optical corner reflector is used as the projectile. The two stations are virtual horizontal planes. When the apex of the corner reflector crosses one or the other of these planes, the end mirror of the vertical arm of the interferometer is conjugated with one or the other of the two mirrors placed in the horizontal arm.

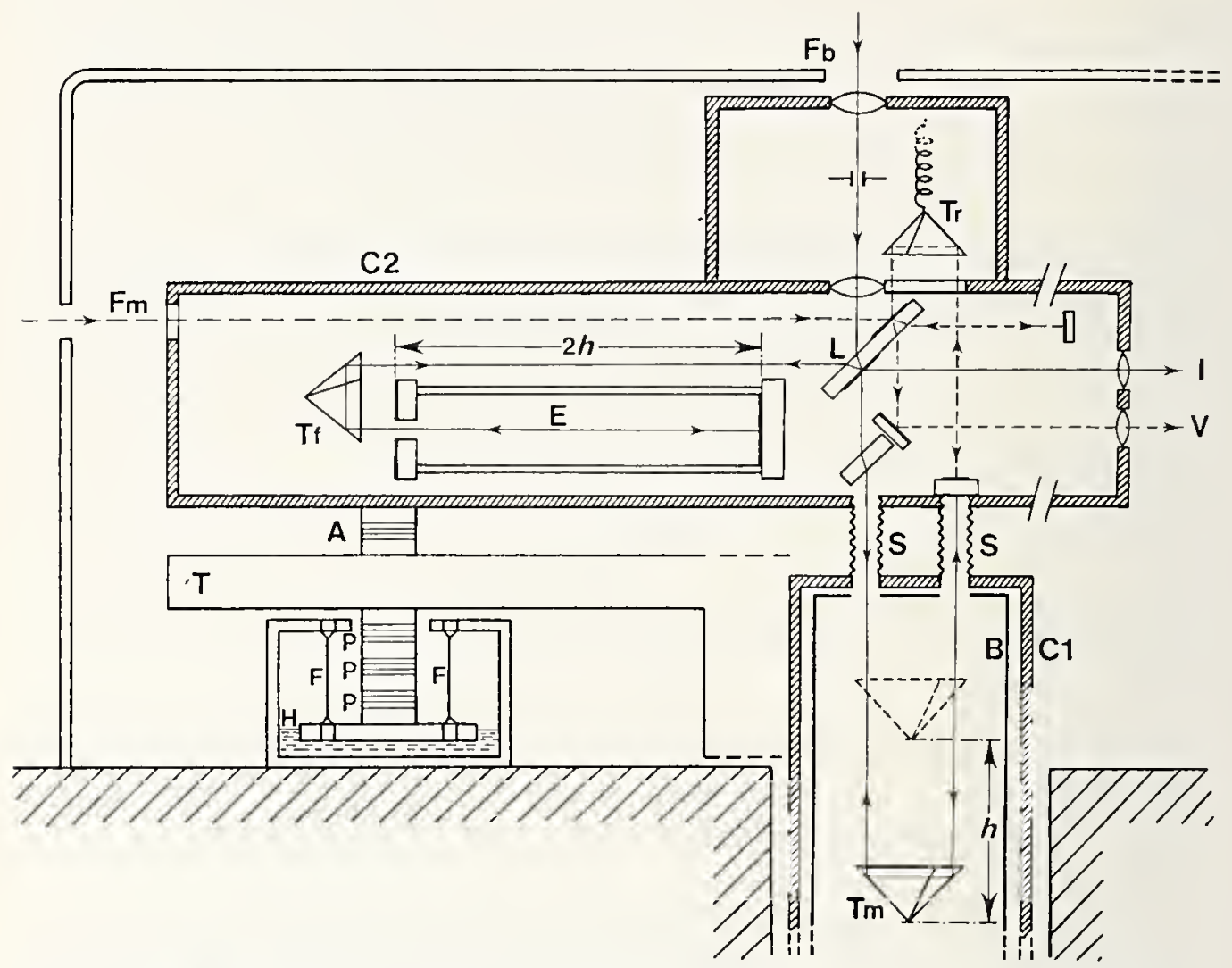

Figure 3. Diagram of the absolute gravimeter of the BIPM. The apparatus comprises two evacuated compartments connected by flexible bellows $\mathrm{S}$. The vertieal compartment $\mathrm{Cl}$ (residual pressure $2 \times 10^{-5} \mathrm{P}$ 'a), provided with a manetic shield $B$, is the place for the ascending then descending, free fall of the molbile eorner refleetor Tm. This is shown at the lower station (full lines) and at the upper station (rlotted) at the distance $h$.

The hrizontal eompartment C2 (residual pressure $1 \times 10^{-2} P^{3}$ a) contains the fixed portions of the measuring interferometer: heam splitter $\mathrm{L}$, fixed eorner refleetor Tf, etalon $\mathrm{E}$, of length $2 h=0.8 \mathrm{~m}$. This interferometer. illuminated by the beam of white light Fl, (full lines) gives, when the mobile reflector passes the lower and upper stations, an interferenee phenomenon I (achromatie fringe).

This eompartment is placed on a platform $\mathrm{T}$ whose stalilization is assured by a wire suspension F, an oil damper H. and the piezoelectric elements P. These last are controlled by the accelerometer $A$ and by the sionals from a seismometer of very long periol support inw the eorner reflector $\operatorname{Tr}$ whose apex constitutes a reference point without appreciable aeceleration. The residual vibrations of $\mathrm{C} 2$ with respect to Tr are recorcled at $\mathrm{V}$ with the aid of an auxiliary interferometer illuminated by the monochromatic light beam $\mathrm{Fm}$ (dashed lines); one takes account of these vibrations in correcting the value of gobtained. 
For these positions, the path difference in the interferometer vanishes and interference fringes can be produced with white light. They are detected by a photomultiplier. The central fringe, or achromatic fringe, is used for starting and stopping the timing counters. By reason of the small width of the beams used and the high frequency of the signal given by the photomultiplier, an ordinary source of white light is not suitable for giving a good signal/noise ratio: one must use a xenon lamp whose flashes are trig. gered at each station passage, at ascent and descent, by means of an appropriate predetection device.

\section{Measurement of Distance}

The length $2 h$ of the etalon constituted by the two fixed mirrors of the horizontal arm of the interferometer is determined by direct comparison with the primary standard radiation of krypton-86 using the same interferometer as for the measurement of $g$. For this measurement, the movable corner reflector is fixed halfway between the two stations. Although the visibility of the interferences thus obtained is weak $(5 \%)$, the precision of $1 \times 10^{-9}$ can be assured. Use of the radiation from a helium-neon laser locked to a saturated absorption line of iodine, whose wavelength is stable to about $1 \times 10^{-9}$ and for which a value has recently been recommended (CCDM, 5th session, 1973), greatly facilitates this measurement and currently permits attaining a precision of $0.7 \times 10^{-9}$.

\section{Projectile and Catapult}

The projectile (fig. 4) consists of six plane mirrors forming two corner reflectors back-to-back at the apex and mounted on a frame of aluminum alloy. Only one corner reflector is used, but this symmetric construction facilitates placing the common apex of the two reflectors in coincidence with the center of gravity of the assembly. The frame is pierced with the openings needed for the passage of the light beam. An axial bore gives passage for the elastic cord which serves for launching. The projectile is accelerated over a distance of $0.30 \mathrm{~m}$ and then follows freely its movement after blocking of the catapult. After a travel of $0.18 \mathrm{~m}$, of $0.05 \mathrm{~s}$ duration, in the course of which its internal oscillations die out, it traverses the lower station and then, after $0.2 \mathrm{~s}$, the upper station. The peak of the trajectory normally occurs $50 \mathrm{~mm}$ above the upper station. In the descent, after a new traversal of the upper and lower stations defining the intervals $t_{1}$ and $t_{2}$, the projectile is braked by elongation of the elastic cord of the catapult.

This cord, in the course of its contraction, develops electric charges. Their effect on the projectile is eliminated by a metallic tube surrounding the cord and forming an electrostatic screen. 


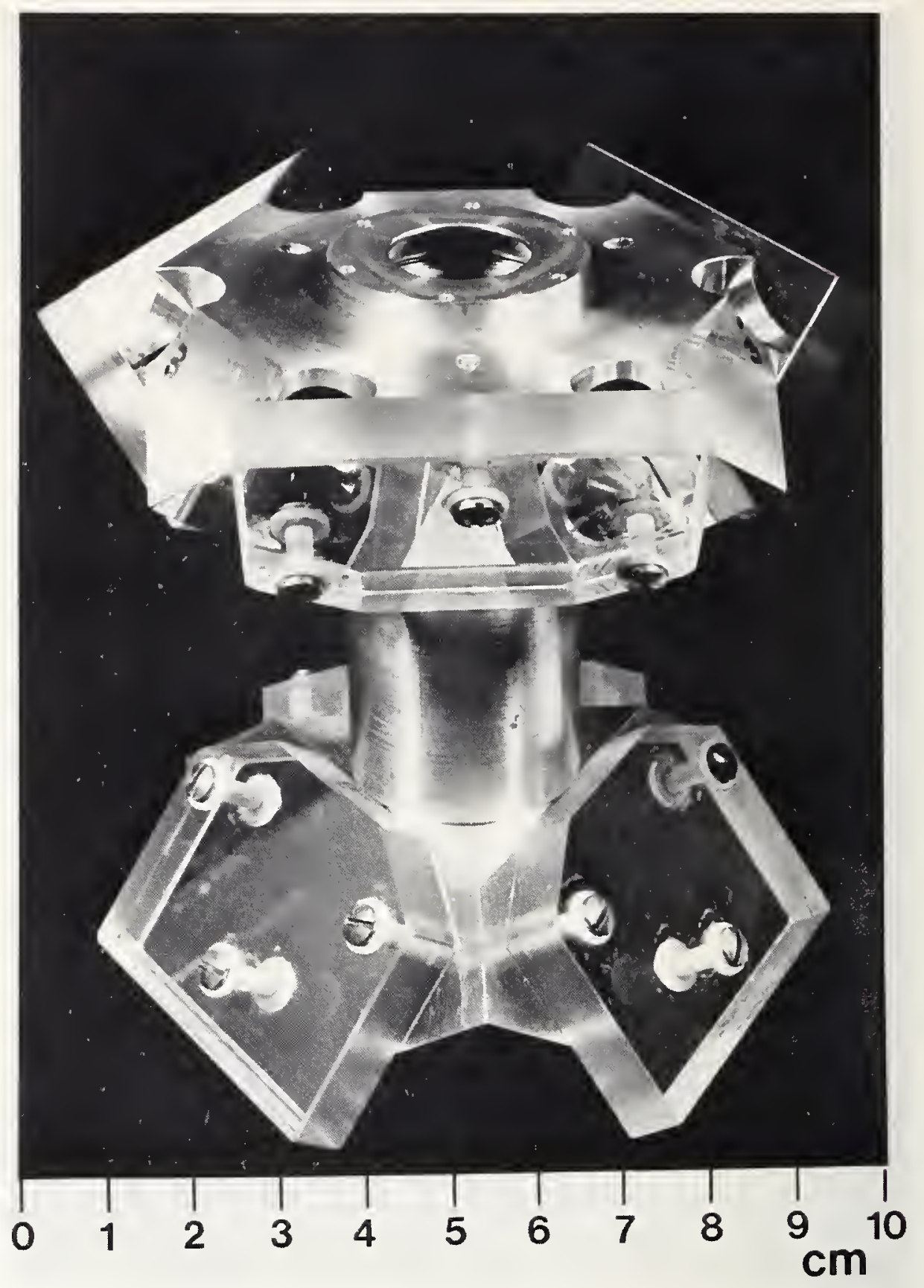

Figure 4. Projertile (mass $430 \mathrm{~g}$ ) for the measurement of $g$ by the two-station method. There are shown the six plane mimors forming two comer reflectors back-to-back at the apex, the openings for the passage of light, and the central hore for the passage of the elastic cord of the catapult. 
The horizontal compartment containing the essential parts of the Michelson interferometer rests on a platform which is protected from vibrations and ground movements by means of various devices.

Suspension by wires and viscous dampers eliminates horizontal vibrations at frequencies above $5 \mathrm{~Hz}$. A set of piezoelectric bearings and a vertical accelerometer of short period constitute a counteracting system which attenuates rapid vertical movements of the grouind $(5$ to $50 \mathrm{~Hz})$. These bearings also carry elements controlled by a vertical seismometer of very low natural frequency and elements permitting the fine adjustment of the horizontality of the platform.

The residual accelerations of the reference system are currently less than about $10^{-8} \mathrm{~m} / \mathrm{s}^{2}$, say $10^{-9} \mathrm{~g}$.

\section{Measurement of Time}

The intervals $t_{1}$ and $t_{2}$ are measured in two steps. A resolution of $50 \mathrm{~ns}$ is obtained by means of counters at $20 \mathrm{MHz}$; then a resolution of $0.1 \mathrm{~ns}$ is obtained by interpolation on a photographic recording of the white light interference fringes; this recording carries time reference marks at a frequency of $20 \mathrm{MHz}$. In practice, the chronometers are preset to measure successively three intervals; $t_{\mathrm{m}}$, the duration of the ascent from the lower station to the upper station, $t_{2}$ already defined, and $t_{\mathrm{d}}$, the duration of the descent from the upper station to the lower station. Thanks to the symmetry of the motion, ascending then descending, the effect of the air resistance is negligible at pressures $p<1 \mathrm{~Pa}$ with the condition that this residual pressure remain constant during the whole displacement. At present, we work with residual pressures of the order of $2 \times 10^{-5} \mathrm{~Pa}$. The difference $\Delta t=t_{\mathrm{d}}-t_{\mathrm{m}}$ then varies linearly as a function of $\mathrm{p}$. If one extrapolates to zero pressure one finds $\Delta t=+5.3 \mathrm{~ns}$, a result conforming perfectly to that foreseen in the case of no braking, taking account of the finite value of the speed of light. This confirms the exactness of the time measurements.

\section{Recording of the Gravitational Tide}

We have seen that the perturbation of $g$ due to the luni-solar effect has for its maximum value $1.6 \times 10^{-6} \mathrm{~m} / \mathrm{s}^{2}$; its rate of variation can reach \pm 0.8 $\times 10^{-8} \mathrm{~m} / \mathrm{s}^{2}$ per minute at the BIPM station. It is necessary to know this perturbation with an uncertainty at most equal to 0.5 percent of its amplitude and at times known to about a minute, or better, to make the measurements of $g$ from day to day consistent with each other. 
The precision of the theoretical determination of the gravitational tide rumning the risk of being revealed insufficient, a recording station equipped with a relative gravimeter has recently been installed (October 1973) at the BIPM. The protection against microseisms and the stabilization of the pressure and temperature have been specially studied, and we can confirm the values of the gravitational tide found with the absolute apparatus. The drift of the relative gravimeter is currently less than $1 \times 10^{-7}$ $\mathrm{m} / \mathrm{s}^{2}$ per month, which is 30 times less than for the best commercial apparatus. The combination of absolute and relative apparatus is favorable to both: the true correction for gravitational tide is now available for the absolute apparatus and this latter permits the calibration of the relative gravimeter and the determination of its long-term drift.

\section{Results and Studies Underway}

The results obtained since 1966, transferred to the reference point of the gravity station of the Pavillon de Breteuil (point A), thanks to a positive relation by means of relative gravimeters, has the mean value $g=9.809260$ $\mathrm{m} / \mathrm{s}^{2}$.

It is seen that the value found in 1958 differs from this by $2.0 \times 10^{-5}$ $\mathrm{m} / \mathrm{s}^{2}$ and that the value in the Potsdam system differs from it by $13.8 \times 10^{-5}$ $\mathrm{m} / \mathrm{s}^{2}$.

This result, joined with those of other laboratories, has led the CIPM to add to the Potsdam gravity system a correction of $-14 \times 10^{-5} \mathrm{~m} / \mathrm{s}^{2}$ (Resolution 1 (1968)).

By reason of the precision attained, this corrected system was soon surpassed. In 1971, the International Union of Geodesy and Geophysics created a "Unified International Gravity Net" (IGSN-71) by adjustment of a large number of absolute and relative measurements of precision at least as good as $10^{-7}$ carried out at various points of the globe. The CIPM adopted this net (Recommendation 2 (CI-1972)) and its future updatings for metrological needs. The BIPM station (point A) is the point where the measurements are the most precise and the systematic errors the most completely studied. It has been chosen as the starting point of this net and as the verification point for transportable absolute measurement apparatus.

Since 1968, the BIPM has given its help to the Istituto di Metrologia $\mathrm{G}$. Colonnetti (Turin) in the construction of a portable absolute gravimeter [9]. The principle of this gravimeter is identical to that of the stationary apparatus of the BIPM (fig. 5) except with regard to the determination of the separation of the two stations which is carried out by the use of reversible counting of interference fringes of a stabilized and calibrated helium-neon laser. On the other hand, the optical reference corner reflector is supported by a vertical seismometer of long period; this avoids registering 

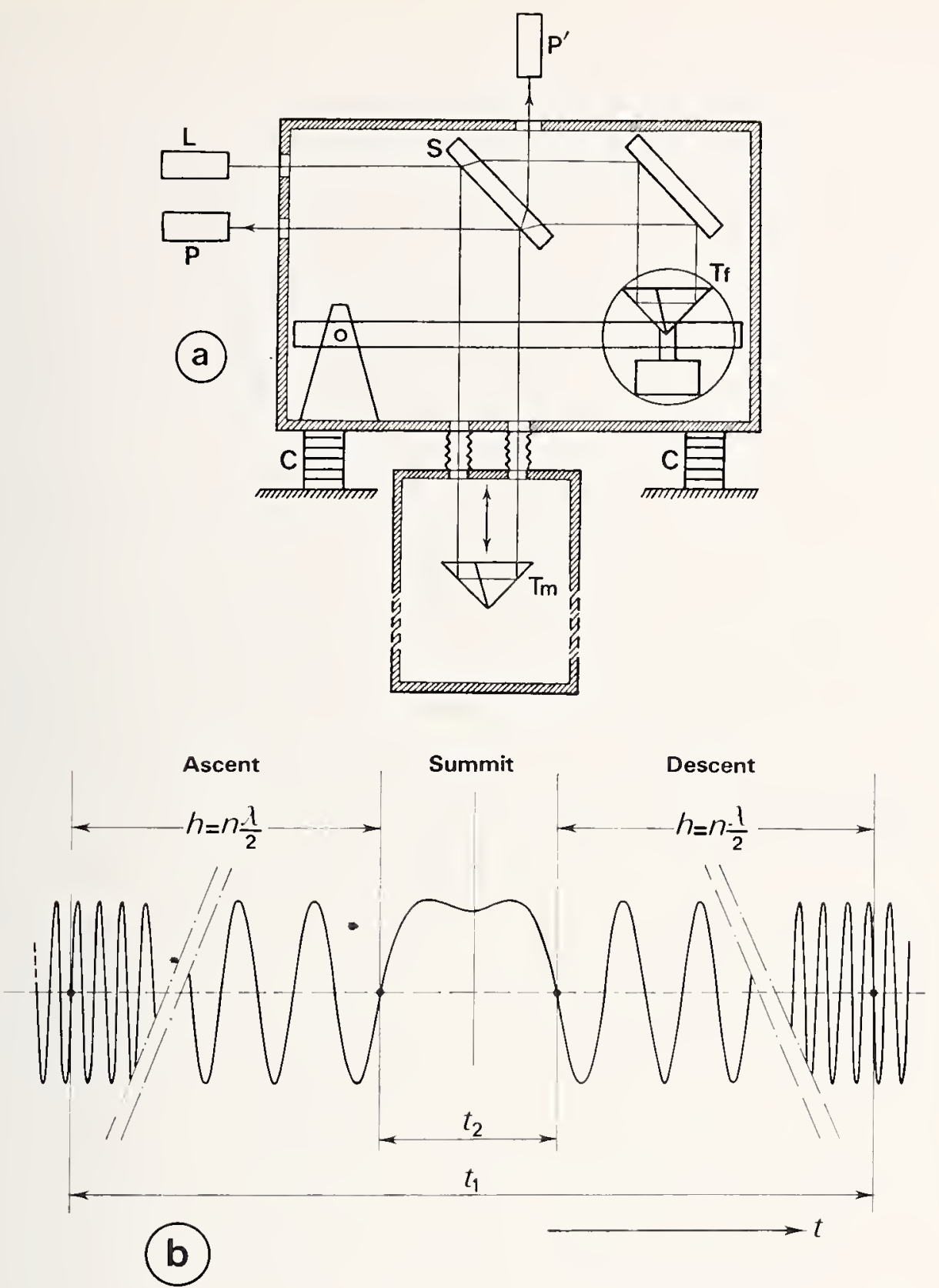

Figure 5. Transportable absolute gravimeter. (a) Diagram of principle. Tm. mobile corner reflector; Tf "fixed" corner reflector supported by a long-periot vertical seismometer; $L$, helium-neon laser emitting a monochromatic raliation of wavelength $\lambda$; $S$, beam sphtter; two photomultipliers $\mathrm{P}$ and $\mathrm{P}^{\prime}$ are used to furnish sinusoidal signals out of phase by approximately one quarter period. which permits reversible counting. The apparatus is supported by piezoelectric bearinos $\mathrm{C}$, elements of an antivibration mounting. (b) Signal from one of the photomultipliers as a function of time $t$. The upper station is defined by the last fringe counted on the ascent and the first fringe counted on the descent. The fower station is defined in the ascent hy the start of the counter at a predetermined instant and in the descent by its retum to $0 ; n$ fringes being counted in the ascent (and deducted in the descent), the distance between the stations is $h=n \lambda / 2$. The intervats $t_{1}$ and $t_{2}$ are measured by counting pulses at a $50 \mathrm{MHz}$ rate. 
microseisms and determining the corrections due to them. Finally, the apparatus operates with a modest vacuum $(1 \mathrm{~Pa})$. which is sufficient to insure an accuracy better than $5 \times 10^{-8} \mathrm{~m} / \mathrm{s}^{2}$; this has permitted simplification of the pumping system. This apparatus, recently tested at the Pavilon de Breteuil, gave for each series of 25 measurements a mean result atreeing with the accepted value to better than $2 \times 10^{-7} \mathrm{~m} / \mathrm{s}^{2}$. It will permit the early realization of numerous gravimetric links, even over larwc distances, without risk of drift. In particular, the difference of the values of $g$ between the stations at Sèvrcs and at Turin (about $0.0039 \mathrm{~m} / \mathrm{s}^{2}$ ), will become the most precise basc for the calibration of relative gravimeters, and its exact knowledge will contribute greatly to the precision of the Unified Intcrnational Gravity Net.

But naturally, measurements made with a fixed apparatus can be more precise than the data of this Net. This is indeed the case for the measurements made at the BIPM. They show up effects which up until now had not been eapable of being demonstrated.

Figure 6 gives some of the values of gobtained since 1966.

It is seen that $g$ is subject to variations reaching $40 \times 10^{-8} \mathrm{~m} / \mathrm{s}^{2}$; their origin is unknown. Some years of study will still be needed to clarify this point which could have important metrological, geophysical, and astronomical consequences.

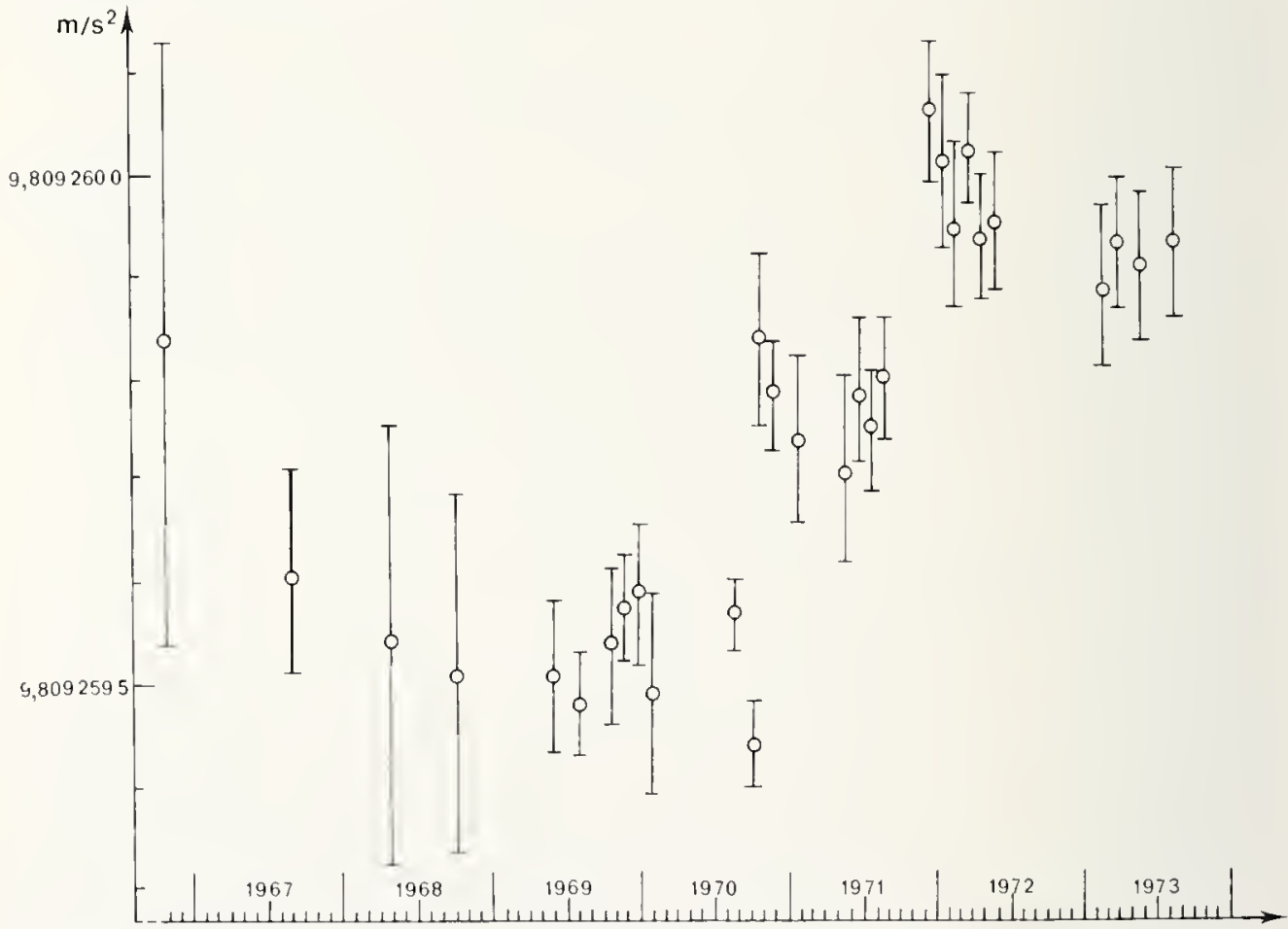

Figure 6. Monthly means of the values of gobtained at the measuring point and transferred to point $A$, reforence point of the BIPV gravity station. Each of these means carries some 50 measurements. The error hars represent the uncertainty of a measurement, calculated from the dispersion of individual results. The transfer from the measuring point to the point $A$ introduees a supplementary uncertainty common to all these values and assessed at $3 \times$ $10^{-8} \mathrm{~m} / \mathrm{s}^{2}$. 


\section{Bibliography}

[1] Defforges, Mesure de l'intensité absolue de la pesanteur, Procès-Verbaux C.I.P.M., pp. 135-182 (1891).

[2] Kühnen, F. and Furtwängler, Ph., Bestimmung der absoluten Grïsse der Schwerkraft zu Potsdam mit Reversions pendeln, Veröff. Königl. Preuszischen Geod. Inst., No. 27, xvi +390 pages, Berlin (1906).

[3] Cook. A. H., The absolute determination of the acceleration due to gravity, Metrologio 1, No. 3, pp. 84-114 (1965).

[4] Volet, Ch., Sur la mesure absolue de la gravité, C.R. Acad. Sc. 222, pp. 373-375 (1946). Volet. Clı. Mesure de l'accélération due à la pesanteur au Pavillon dle Breteuil, C.R. Acad. Sc. 235. pp. 442-444 (1952).

[5] Thulin, Å., Détermination absolue de l'accélération due à la pesanteur au Pavillon de Breteuil, Travaux et Mémoires du B.I.P.M. 22 (1), 91 pages (1961).

[6] Volet, Ch., L'intensité de la pesanteur déterminée par la chute d'un corps, C.R. Acad. Sc. 224, pp. 1815-1816 (1947).

[7] Sakuma, A., Recent developments in the absolute measure of gravitational acceleration, Nat. Bur. Stand. (U.S.), Spec. Publ. 343, pp. 447-456 (August 1971), and Recueil de Travaux du B.I.P.M. 3, article 15 (1971-1972).

Sakuma, A.. A permanent station for the absolute determination of gravity approach. ing one microgal accuracy, Proc. Symposium on Earth's gravitational field and secular variations in position, University of N.S.W., Sydney, pp. 674-684 (1973), and Recueil de Travaux du B.I.P.M. 4, article 11 (1973-1974).

[8] Procès-Verbaux C.I.P.M., p. 49 (1960).

[9] Cerutti, G., Cannizzo, L.., Sakuma, A., and Hostache. J., A transportable apparatus for absolute gravity measurement, VDI-Berichte 212, pp. $49-51$ (1974), and Recueil de Travaux du B.I.P.M. 4, article 12 (1973-1974). 



\section{CHAPTER V}

\section{MANOBAROMETRY}

From the beginning of the BIPM, the responsible scientific leaders recognized the necessity of an apparatus which permits the precise evaluation of the atmospheric pressure (barometer) or, more generally, of other pressures (manometer) and, less than 10 years after its creation, the BIPM constructed a quality manobarometer (fig. 1); this apparatus competed successfully, practically without essential modification, with the various developments which appeared successively in the world in the course of the first half of the $20 t h$ century. It was a mercury apparatus with several original features, the first manometer, for example, to have large diameter menisci (about $37 \mathrm{~mm}$ ) rendering negligible the influence of capillary deformation. The vertical distance between the central parts of the two menisci was determined optically, by transfer to a vertical calibrated scale with the aid of the two horizontal telescopes of a cathetometer (fig. 2); to define the surface of a meniscus, the optical procedure adopted as early as 1890 , already very much elaborated, utilized the middle point between a reference (glass point placed inside the chambers of the manometer) and its image reflected by the mercury. Furthermore, the apparatus had been filled with mercury by distillation under vacuum [1].

It is rather difficult to estimate with what accuracy one can determine a pressure with this apparatus. The pressure $p$ is deduced from the relation $p=h \rho g$, where $h$ is the level difference between the two mercury menisci, $\rho$ the mass density of the mercury, and $g$ the acceleration of gravity. Even aside from the optical and mechanical errors which affect the measurement of the length $h$ and which prevent a precision better than $10 \mu \mathrm{m}$ (or about $10^{-5}$ of the at mospheric pressure), the two other factors $\rho$ and $g$ were not known to better than about $10^{-5}$, and this was so at least until around 1930.

In practice, it was necessary to await the years after 1950 for profound metrological studies based on the rapid progress of technology to improve, almost simultaneously, the knowledge of $\rho$ and $g ; \rho$ is now known to about $10^{-6}[2]$ and $g$ to better than $10^{-7}[3]$. We could therefore envisage improving the measurement of pressure. conditioned, however, on conceiving a new manobarometer in which the determination of the height of the mercu- 
ry would be possible to about 1 in $10^{4}$. The study of a new apparatus was therefore undertaken, because the reatization of a more precise temperature scale, based on fixed point s corresponding to the boiling temperatures of pure substances, demanded a knowledge of pressure to a few parts in $10^{6}$. And even today when the progressive abandonment of boiling points renders the problem less crucial in thermometry, the precise measurement of pressure becomes necessary in other areas, for example in the comparison of mass in all the cases where air pressure plays a role.

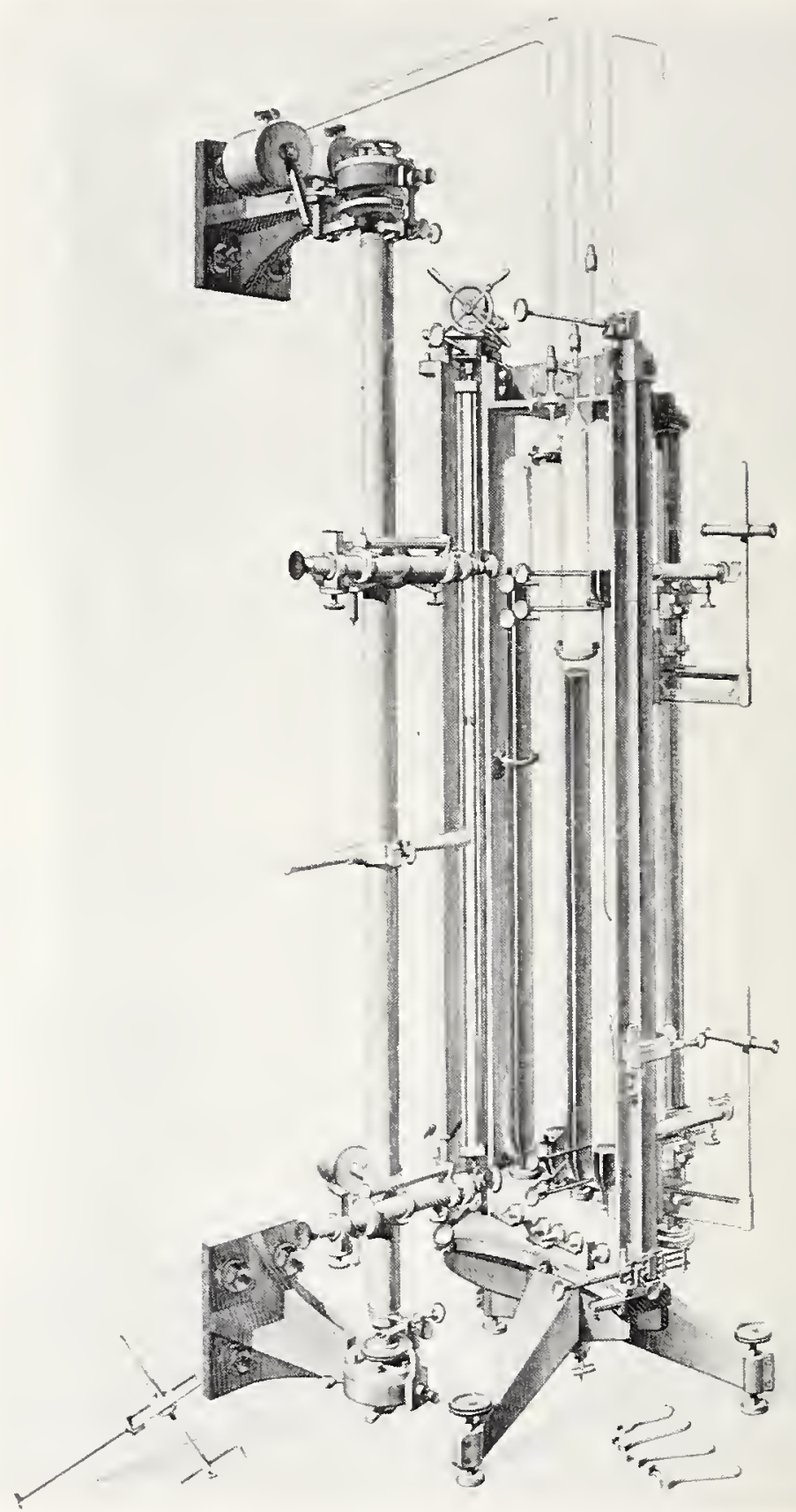

Figure 1. General view of the standard manobarometer used at the BIPII from 1884 to 1966. 


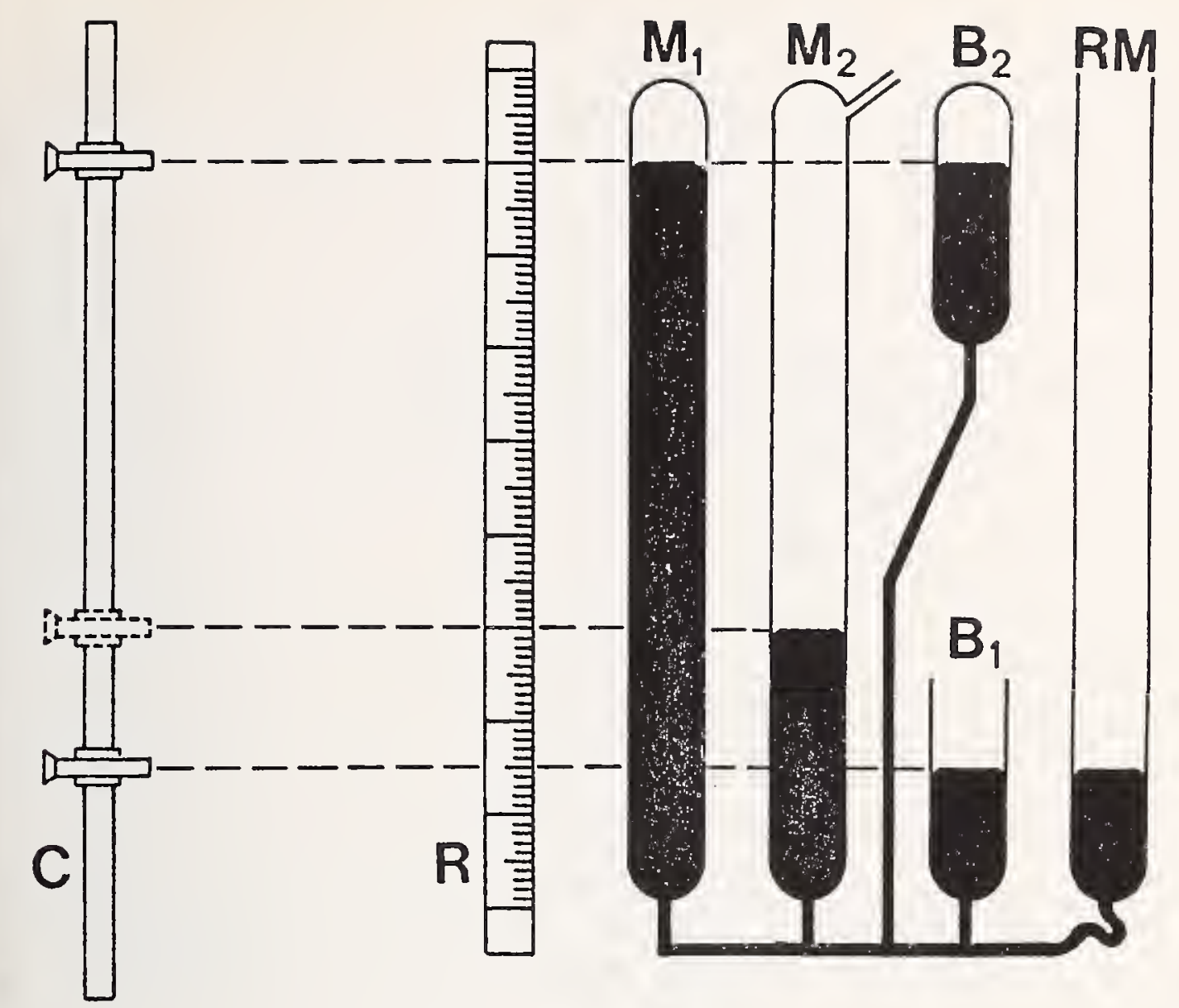

Figure 2. Diagram of the standard manobarometer of 1884. C. cathetometer; R, graduated scale: $\mathrm{M}_{1}, \mathrm{M}_{2}$, chambers of the manometer: $\mathrm{B}_{1}, \mathrm{~B}_{2}$, chambers of the barometer; $\mathrm{RM}$, mercury reservoir the four chambers and the scale are at the same distance from the cathetome tel*).

\section{Interferometric Manobarometer}

The apparatus studied at the BIPM and put into service in 1966 [4] is still a mercury manobarometer. Mercury is still the most suitable liquid: it can be obtained very pure, its thermal conductivity (that of a metal) is good, and its mass density is high. The originality of this apparatus resides in the device for localizing the mercury surfaces, which allows substituting for the measurement of a not directly accessible vertical distance, the easy measurement of a horizont al displacement (fig. 3).

This device utilizes the interference of light, not for measuring the distance which separates the two mercury menisci, but simply as an optical feeler; for this, one depends on the behavior of white light interference fringes which appear when the two optical paths are sensibly equal (to about $1 \mu \mathrm{m}$ ). The two mercury surfaces are used as mirrors in a Michelson interferometer; the optical path difference which appears when there is a 


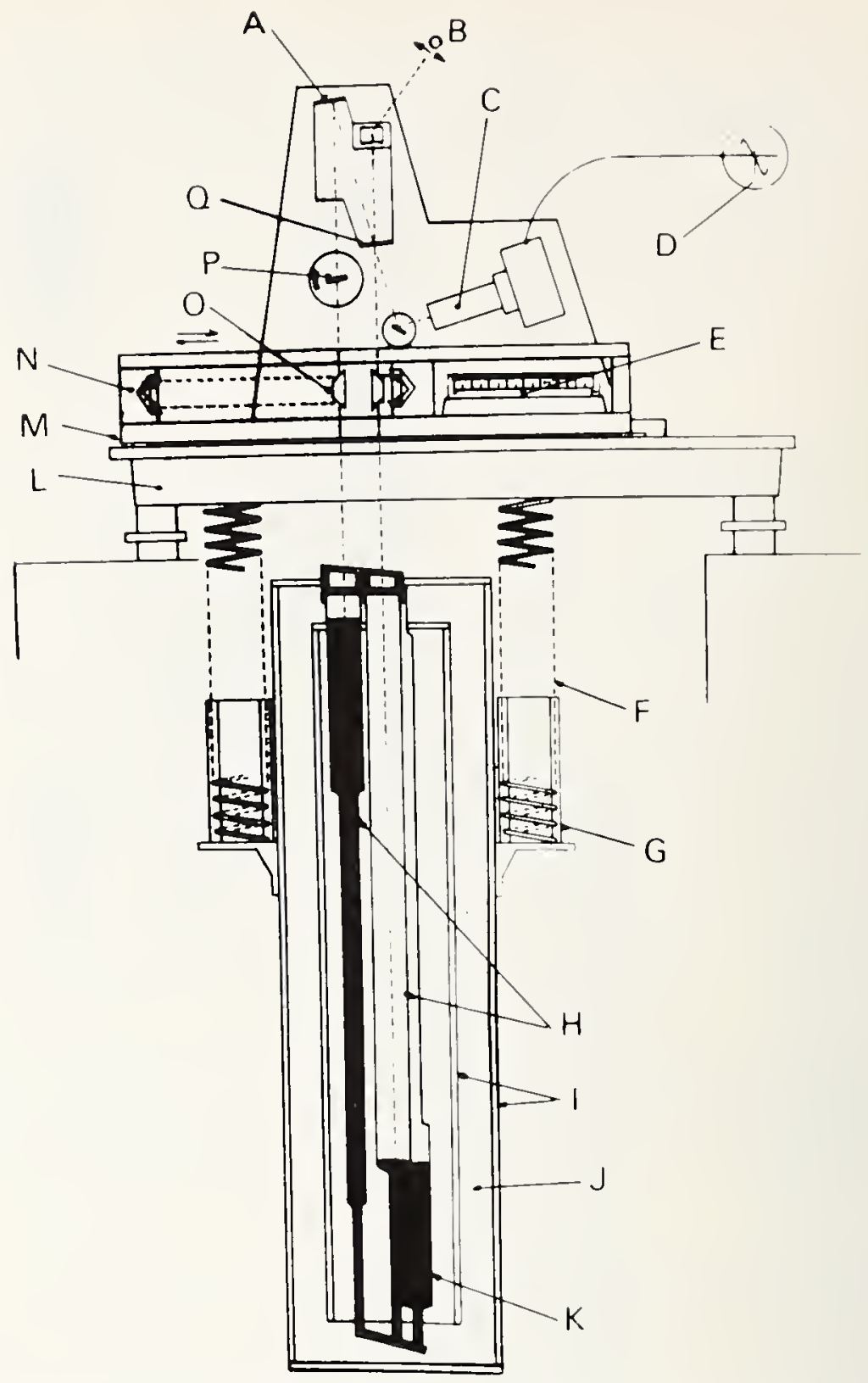

Figure 3. Diagram of the standard manobarometer placed in service at the BIPM in 1966. A. reference mirror: B. source of white light: C. photomultiplier: D. uscilloscope: E gratuated scale: F, suspension spring: (, anmlar oil damper: H. measurement chamber; I. aluminum envelopes: J. thermal insulation: $K$, reservoir chamber: L. supporting frame: M, carriage: N. uptical comer reflector: O, uptical reflecting prism: P. uscillating compensator plate: Q. beam splitter.

The measurement of a pressure comprises two operations. First, one evaeuates the two measurement chambers and displaces the carriage bearing the two concr reflecturs and the scale to the puint of obtaining fringes on the oscilloscope screen: a reading of the scale by means of a microscope provided with a micrometer then furnishes the "zero" of the apparatus. One of the chambers is then put in communication with the clused space in which one wishes to know the pressure and. by a procedure analogous to the preceding. a second reading on the scale is obtained. The differcnce of the $\mathrm{t}$ wo readings gives, after various corrections, the level difference between the two mercury menisci. 


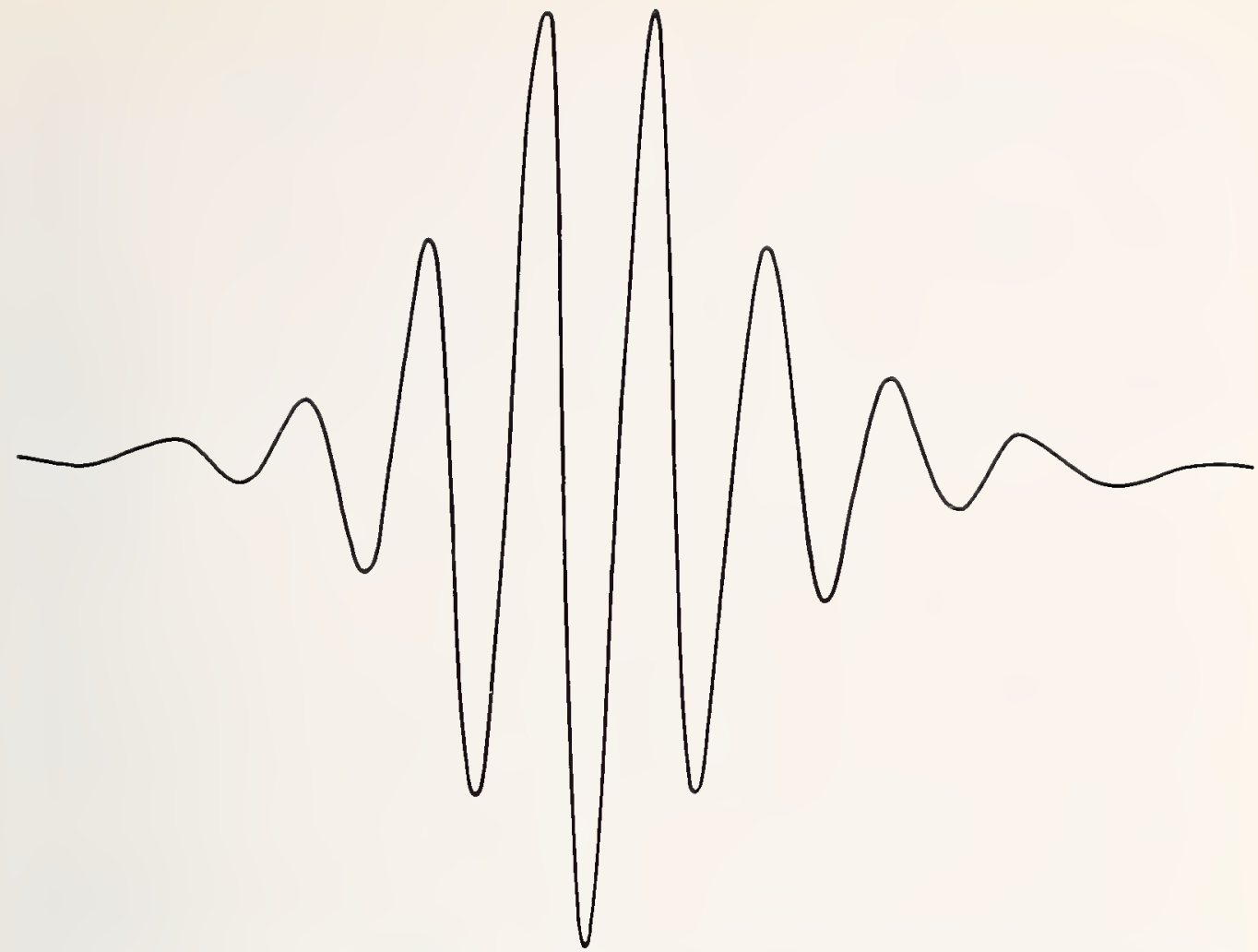

Figure 4. Image observed on the oscilloscope screen. When one observes a packet of several fringes, approximately centered on the oscilluscope screen, the arbustment is reached the length of the packet corresponds to a carriage displacement of less than $1 \mu \mathrm{m}$ ).

change in the relative positions of the two menisci, that is to say, when the pressure is changed, can be compensated by the displacement of two solid optical elements; this displacement, measured on a calibrated scale, indicates the change of height of the mercury. The interference fringes are observed on the screen of an oscilloscope (fig. 4) whose sweep is synchronized with the oscillatory movement of the compensation plate of the interferometer; this movement creates the small variation of path difference needed for exploration of the fringes.

Some corrections are, however, necessary. For example, the use of light interference involves the indices of refraction of the different media traversed by the light; now the ambient air and the nitrogen contained in the measuring chamber have slightly different indices of refraction and are not equally dispersive; it results from this that, in general, equality of the optical paths does not rigorously correspond to equality of the geometrical paths (fig. 5). The correction term, a function of the pressure to be measured, and easily calculated, never exceeds $5 \mathrm{~Pa}$, however. The manobarometer thus permits measuring pressure in the entire range from 0 to a little above $10^{5} \mathrm{~Pa}$, with an accuracy of 0.1 to $0.2 \mathrm{~Pa}$, that is to say, to about 1 in $10^{6}$ in the neighborhood of atmospheric pressure. 

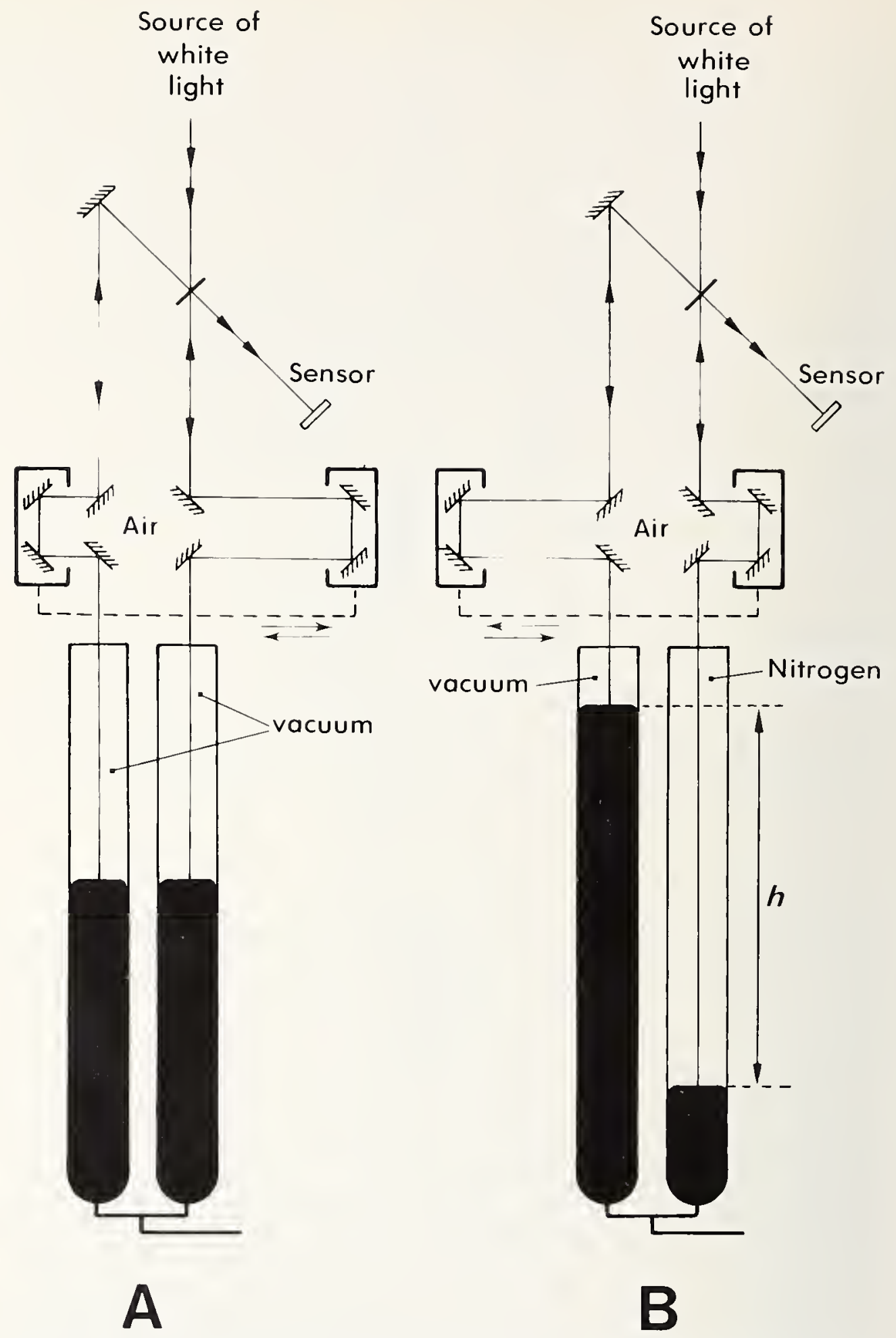

Figure 5. Paths of the light beams in the interferometer. A: for a zero pressure: B: for an arbitrary pressure, $p$. Note that the perfect symmetry of the optical paths in the first case no longer exists in the second (in fact. one compensates the difference by a difference of pathlengths in air). 
The practical realization of the apparatus has naturally required great precautions. In order to utilize the mercury menisci as mirrors, it is necessary to protect the manometer chambers from vibrations; these exhibit themselves by ripples on the mercury surface which weaken the contrast of the interference fringes; observations then become difficult without. however, any risk of systematic error. We have sufficiently attenuated the perturbing vibrations at frequencies above a few hert $z$ by using a damped elastic suspension of very low resonance frequency (helical springs and oil dampers) and by connecting the manometer chambers to the fixed pipes with flexible links.

For the mercury to retain its initial properties, it is necessary to protect against all contamination and, in particular, to avoid exposing it to contact with the humidity of the air; the manometer chambers are kept under an artificial atmosphere of dry nitrogen. Up until now, the manobarometer has been used only for comparative measurements, tied to a closed space containing dry nitrogen, but nothing would prevent interposing a mem. brane for particular applications (gas thermometry, for example).

Another very important problem concerns the mercury temperature which should be very uniform and known to better than about $0.01 \mathrm{~K}$, if one wishes to know the density of the mercury to better than 10-6; a double envelope of polished aluminum enclosing a large thickness of a good thermal insulating material assures the required uniformity of temperature; an ensemble comprising a mercury thermometer and several differential thermocouples allows determining the temperature at a reference point and the temperature differences between this point and the mercury at different levels.

This manobarometer, as it operates at the BIPM, permits either the measurement of a pressure or the adjustment of a pressure to a predetermined value, in a few minutes.

An apparatus of this type not being transportable, there appears a need for pressure transport standards of an equivalent precision; it has already been proposed, for example, to use for reference the vapor pressure of the triple points of various pure substances. These standards would permit an interesting comparison of some absolute manobarometers which are in service in the large metrology laboratories. 


\section{Bibliography}

[1] Marek, W. J.. Pesćes exécutées au B.I.P.M. du ler octobre 1881 au 15 janvier 1883 , Traucux et Mémoires B.I.P.M. 3, (1884), see Instruments auxiliaires, Baromètres, pp. D22-D51 (1884).

[2] Cook, A. H.. Precise measurements of the density of mercury at $20^{\circ} \mathrm{C}$, Phil. Trans. Roy. Soc. 254 A. pp. 125-154 (1961).

[3] Acceleration of gravity: this volume. p. 103.

[4] Bonboure, J. and Terrien, J., The new standard manobarometer of the Bureau International des l'oids et Mesures, Metrologia 4, pp. 59-68 (1968).

Bonhoure, J., Manobaromètre pour la mesure absolue des pressions. Entropie 13, pp. 57-61 (1967), and Recueil de Travaux du B.I.P.M. 1 (1966-1967). 


\section{CHAPTER VI}

\section{THERMOMETRY}

\section{The Gas Thermometer and the Mercury Thermometer}

Measurements of length and mass, which were originally the principal preoccupation of BIPM, require a good knowledge of the temperature; this explains the almost simultaneous development, starting in 1875, of important studies on the gas thermometer and the mercury thermometer [1].

The work undertaken utilizing successively hydrogen, nitrogen, and carbon dioxide in the thermometer quickly showed that hydrogen was the gas closest to the ideal state, and as early as 1887 the CIPM adopted as the standard thermometric scale, the centesimal (or centigrade) scalc of the hydrogen thermometer. This scale was based on two fixed temperature points to which were assigned the values 0 and 100: the temperature of melting ice and that of the vapor of water boiling under standard atmospheric pressure.

For practical applications, however, there was needed a measuring in strument which would be more convenient to use than the gas thermometer. Although in 1875 the reputation of the mercury thermometer was not excellent, the BIPM decided to evaluate its possibilities. Techniques were developed very rapidly; they consist in determining very carefully all the corrections necessary to bring the readings into agreement with those which a perfect instrument would give: they permitted reliable and precise use of the mercury thermometer. The disappearance, about 1925, of the "hard glass" used up until then for the construction of thermometers, almost brought the question up again and almost nullified the value of the enormous work which had been accomplished; but the adoption of glasses of equivalent quality (Jena glass $16^{I I I}$ for the reservoir, "green glass" for the stem) saved the situation. Further progress, which brought the mercury thermometer to a high degree of perfection, was marked by two important stages. In 1933, the double-graduation stem (front and rear) permitted the suppression of parallax error; in the next few years, the feasibility of replacing glass by fused quartz made possible the elimination of a limitation of the mercury thermometer, the instabilities of the zero: "slow climb" due to aging of the glass and "depression" due to residual dilatation of the glass. It is generally admitted that the mercury thermometer, used under 
the best conditions and by an experienced observer, can give a precision of 2 or $3 \mathrm{mK}$. Since 1887, therefore, mercury thermometers which have been carefully evaluated and compared to a hydrogen thermometer could provide a convenient and precise representation of the standard thermometric scale.

\section{The International Practical Temperature Scale}

Some discussion of the chronological evolution of this practical scale of temperature [2] is necessary to establish the orientation of the work of BIPM.

Since the beginning of the century, the need for an international agreement on a practical temperature scale had been felt; a draft presented by the United States of America and soon supported by Germany and Great Britain led to the adoption of the "International Temperature Scale of 1927." This scale, defined only above - $190^{\circ} \mathrm{C}$, was based on six reproducible equilibrium temperatures to which were assigned numerical values, and on instruments and interpolation procedures: the platinum resistance thermometer up to $660{ }^{\circ} \mathrm{C}$, the platinum/platinum -10 percent rhodium thermocouple up to the freezing point of gold $\left(1063^{\circ} \mathrm{C}\right)$, and the Wien radiation law above that. The scale agreed with the thermodynamic scale as well as possible and as far as the knowledge of the time permitted its verification.

Some modifications were made to the scale in 1948, in 1960, and in 1968 to arrive at the scale which is currently in force under the name "International Practical Temperature Scale of 1968"; these had been primarily for the purpose of extending the scale to low temperatures (down to 13 $\mathrm{K}$ ), of replacing the melting point of ice by the more reproducible triple point of water and of substituting Planck's ${ }^{1}$ law for that of Wien. Moreover, account had been taken of the increase in the accuracy of measurements, and the international practical scale was adjusted so that it gives, within the limits of this accuracy, an approximation as close as possible to the thermodynamic temperature (fig. 1).

Note moreover that there is no longer a thermodynamic scale, since the kelvin $(\mathrm{K})$, which is the unit of thermodynamic temperature $(T)$, is defined as being the fraction $1 / 273.16$ of the thermodynamic temperature of the triple point of water (1954, 10th CGPM). Concurrently, there exists

\footnotetext{
${ }^{1}$ Planck's law gives the spectral density of the energy exitance $M$ of a blackbody as a function of the wavelength $\lambda$ and the temperature $T$ :

$$
M=c_{1} \lambda^{-5}\left\{\exp \left(c_{2} / \lambda T\right)-1\right\}^{-1}: c_{1} \text { and } c_{2} \text { are two constants. }
$$

Wien's law is obtained by neglecting 1 compared to the exponential. The exitance is the quotient of the flux leaving a surface element of the blackbody, by the area of that element.
} 


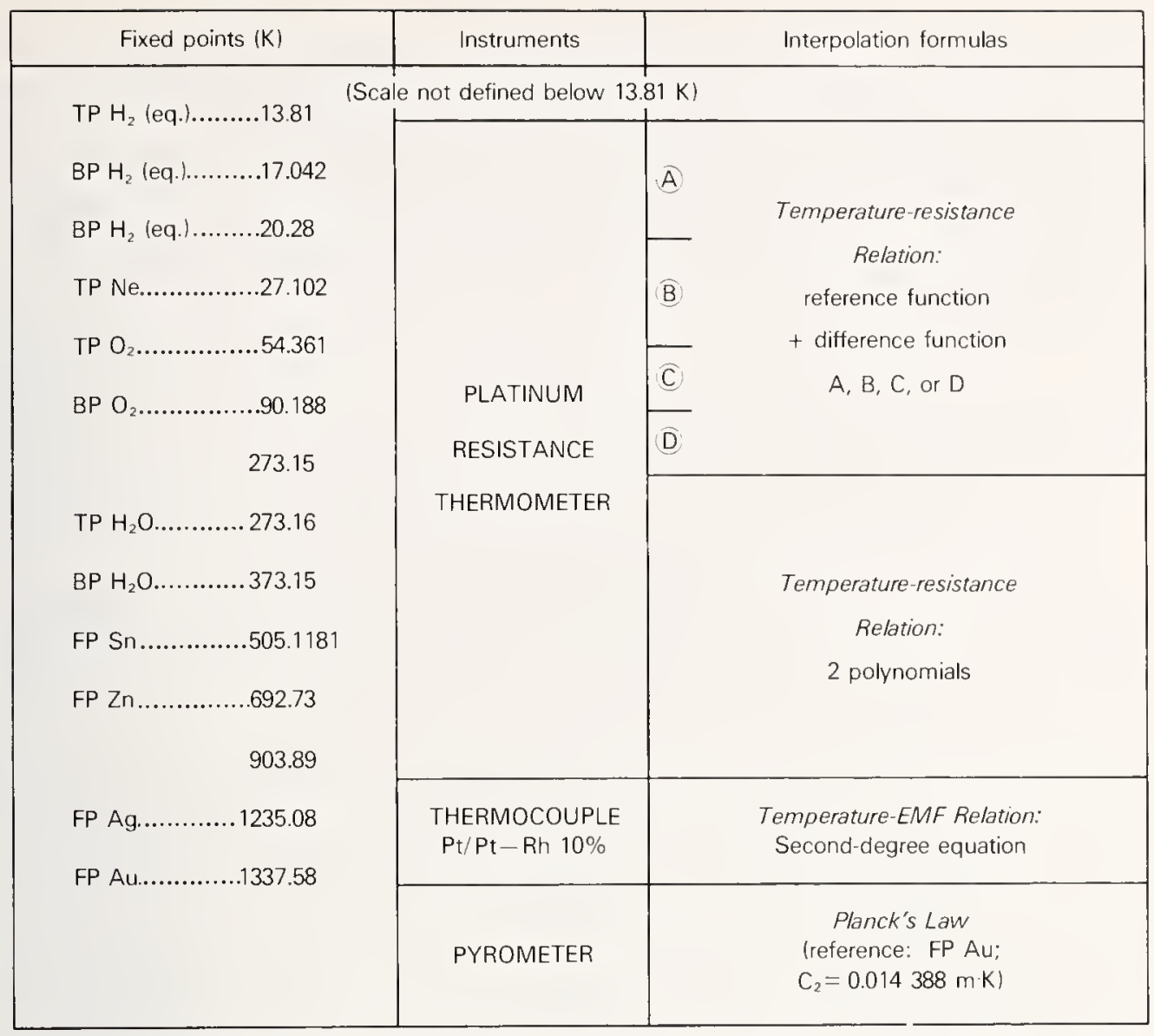

Figure 1. Principal elements defining the International Practical Temperature Scale of 1968 (IPTS-68). TP. triple point: FP, freezing point: BP. boiling point under a pressure of 101325 $\mathrm{Pa}$ : BP*, boiling point uder a pressure of $33360.6 \mathrm{~Pa}$; eq., signifies that the hydrogen has its wrtho-para composition in equilibrium at the temperature considered.

The instruments should be standardized at the fixed temperature puints; at intermediate temperatures. the interpolation formulas relate the temperature to the instrument indicatiin.

a Celsius temperature $(t)$, obtained by shifting the zero and defined by $t=$ $T-273.15 \mathrm{~K}$, whose unit is the degree Celsius $\left({ }^{\circ} \mathrm{C}\right)$ which is equal to the kelvin. It follows that the temperature of the triple point of water is also $0.01{ }^{\circ} \mathrm{C}$, by definition.

The mercury thermometer has never figured as an interpolation instrument in the International Scale, even in the range of amhient temperatures; the platinum resistance thermometer has always been preferred because it is more precise, and also because the same instrument can cover a very large temperature range. Since 1897, the BIPM has been interested in this latter type of thermometer and has studied its properties up to $600{ }^{\circ} \mathrm{C}$ by direct comparison with a gas thermometer; it verified the resistance-temperature relation proposed by Callendar and Griffiths. Incidentally, in the course of this work, the BIPM even assigned a value to the boiling point of sulfur, a fixed point which was used much later, from 1927 to 1960, as a reference in the International Scale. But later and for 
various reasons, the activity of BIPM in this area declined and, as paradoxical as it may appear, it was necessary to wait until 1960 for the beginning of the installation at BIPM of the necessary equipment for the realization of the International Scale. Today, this installation operates from $0{ }^{\circ} \mathrm{C}$ to $1064{ }^{\circ} \mathrm{C}$ with its greatest precision, and it is in operation below $0{ }^{\circ} \mathrm{C}$, both to satisfy BIPM's own needs and to respond to calibration requests from national laboratories.

\section{Measurement Equipment of the BIPM}

The installation includes not only the defined fixed points and the specified interpolation instruments, but also measuring apparatus.

The fixed points actually realized are the triple point of water $\left(0.01{ }^{\circ} \mathrm{C}\right)$ and the freezing points of tin $\left(231.9681^{\circ} \mathrm{C}\right)$, of zinc $\left(419.58{ }^{\circ} \mathrm{C}\right)$, of silver $\left(961.93^{\circ} \mathrm{C}\right)$, and of gold $\left(1064.43^{\circ} \mathrm{C}\right)$; the boiling point of water, less precise, has been abandoned.

The technique for use of a water triple-point cell (fig. 2) is now well established. An ice mantle of well-regulated thickness is obtained around the thermometer well by introducing a very closely fitting metallic rod, previously chilled in liquid nitrogen; fewer than 10 such operations suffices, which requires only a very short time, but it is necessary to wait several hours if one wishes the thermal equilibrium of the triple point to be attained to better than $0.000 \mathrm{l} \mathrm{K}$. This equilibrium can be preserved for

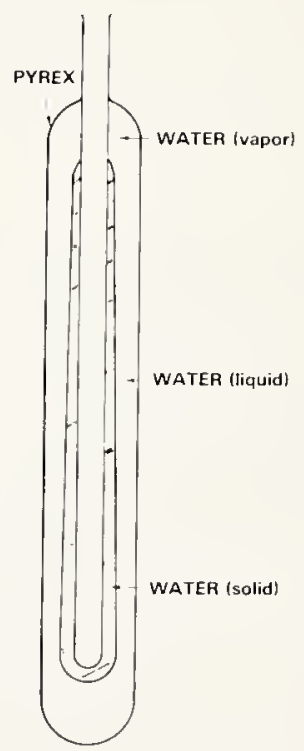

Figure 2. Cell for the triple point of water. The temperature $0.01{ }^{\circ} \mathrm{C}$ is obtained, with water of very high purity contained in a sealed ampoule. wherever the ice is in equilibrium with the liquid-vapor interface. The ampoule should therefore be free of all gas and all foreign substance. 
several weeks if one takes the precaution of placing the cell in a Plexiglas tube which is itself kept in a tub of crushed ice.

To realize the freezing points (figs. 3 and 4), we have researched the techniques which allow the best reproducibility, for example by blowing argon into the thermometer well to initiate crystallization in the case of a metal which undergoes an appreciable supercooling (tin).

Similarly, crucibles are kept in an argon atmosphere to avoid oxidation of the graphite and, in the case of silver, the solution of the oxygen of the air into the molten metal. As to ovens, they have a large time constant (greater than 10 hours) and multiple (five) heater windings, which permits obtaining a very high stability and a very good uniformity of temperat ure throughout the crucible $(0.01$ to $0.02 \mathrm{~K})$.

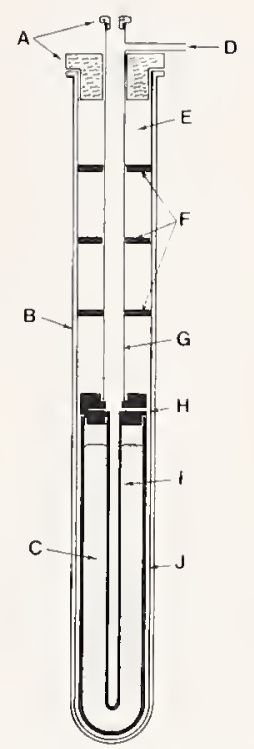

Figure 3. Diagram of a cavity containing a pure metal, for the realization of a freezing point. A, Teflon stoppers; B, guard cavity; C, pure metal; D, argon inlet; E, thermal insulation; F. graphite disks; G, thermometer guide; H, graphite lid; I, graphite thermometer well; J. graphite cavity.

A step in the curve of temperature as a function of the time corresponds to the solidliquid equilibrium of a very pure metal; one can thus realize very reproducible temperature.

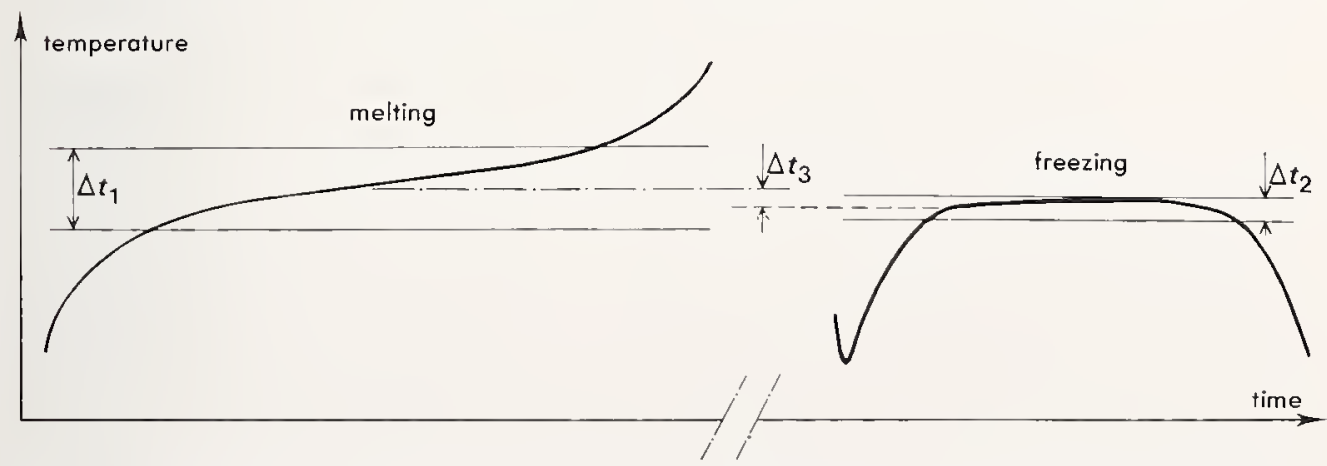

Figure 4. Steps of the freezing and melting of a metal. The form of the steps (in particular the temperature interval $\Delta t_{1}$ of the fusion step) and the lowering of the temperature of the freezing step, $\Delta t_{3}$, give information on the purity of the metal. In the ideal case, $\Delta t_{1}, \Delta t_{2}, \Delta t_{3}$ would be zero. 


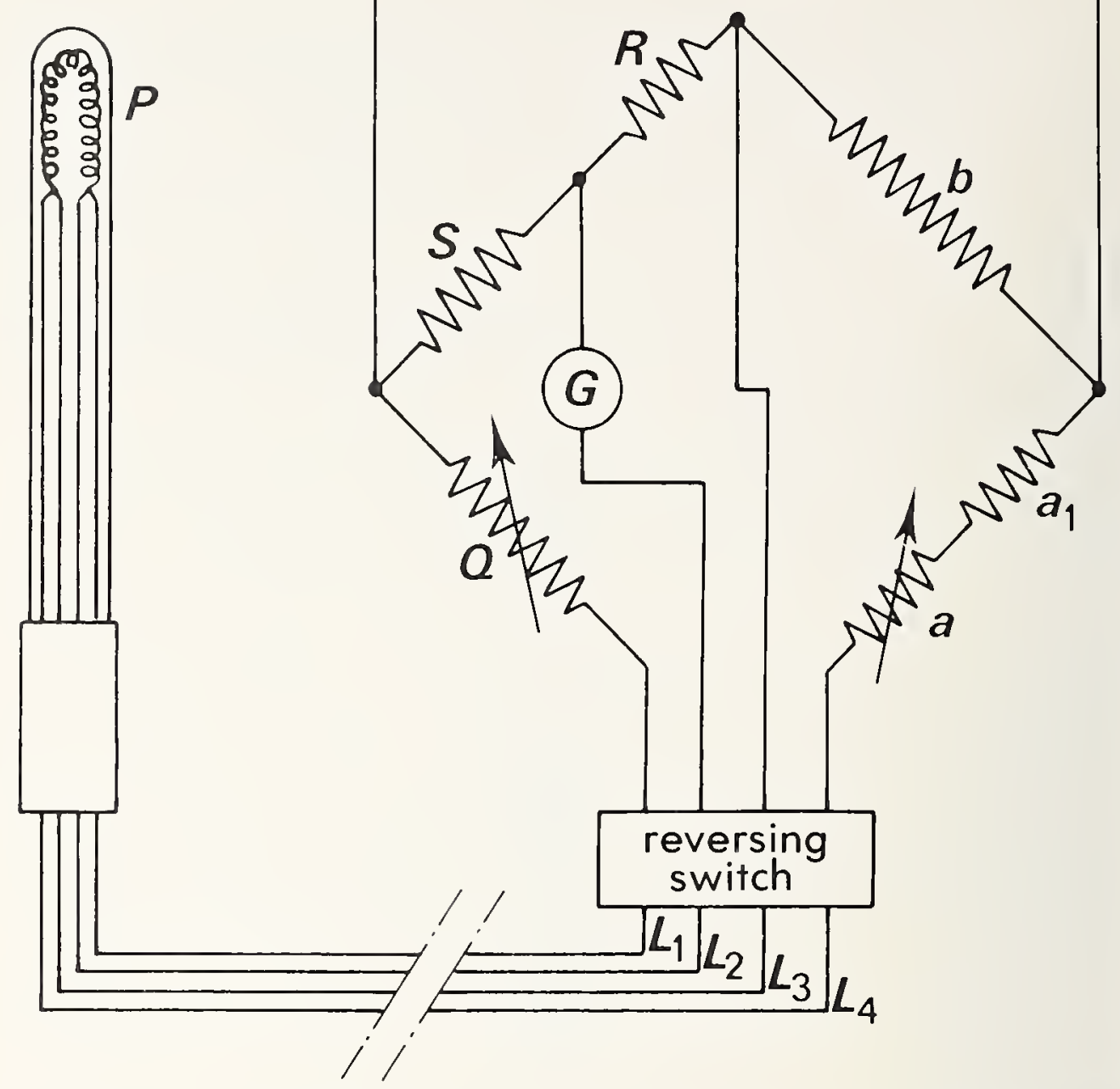

Figure 5. The Smith bridge and the measmement of resistance thermometers. The nominal values of the bridge resistances are: $S=1000 \Omega, K=10 \Omega, b=990 \Omega, a_{1}=10 \Omega ; a=Q$. The lead resistances $L_{1} . L_{2,}, L_{3,3}, L_{4}$ are almost equal (mean value $L_{\text {) }}$.

If the preliminary arljustment of the bridge is such that

$$
a+a_{1} / Q+R=b / S-R
$$

one has, at balance:

$$
P=(R / S)\left[Q+\left(L_{3}-L_{2}\right)+\frac{L^{2}}{b+a_{1}+Q}\right]
$$

One can. by interchange of the leads $L_{1}, L_{2} . L_{3}, L_{4}$ with $L_{4}, L_{3}, L_{2}$. $L_{1}$. get rid of the resistances of the conductors, up to a mean value $L$ which is a function of the precision desired for $P$ or equivalently for the temperature. In practice, temperature measurements are possible to ahout $10^{-4} \mathrm{~K}$ for $L \leqslant 2 \Omega$. which allows heeping the Smith bridye at a fixed location even for measuring thermometers as far as $100 \mathrm{~m}$ away from the bridge. 
The interpolation instruments differ on the two sides of $630.74{ }^{\circ} \mathrm{C}$ : the resistance thermometer below, and the platinum/platinum - 10 percent rhodium thermocouple above. The BIPM possesses some resistance thermometers of recent fabrication: the resistor, of very pure platinum, is enclosed in an envelope containing dry air; the thermocouples are completely mounted and stabilized at the BIPM, starting with wire of suitable characteristics and purity and with tubes of pure alumina.

The measuring apparatus comprises a potentiometer of $0.1 \mu \mathrm{V}$ resolution for the thermocouples, a resistance comparator using alternating current (resolution of few parts in $10^{7}$ ) and a type III Smith bridge (resolution $10^{-5} \Omega$, corresponding to $10^{-4} \mathrm{~K}$ for a thermometer having a resistance of 25 $\Omega$ at $0{ }^{\circ} \mathrm{C}$ ) for the resistance thermometers. The type III Smith bridge (fig. 5 ) is a modified version of the Kelvin double bridge; it is particularly well adapted to measuring resistance thermometers because it permits, by interchange of connections, the almost perfect elimination of the resistance of the conductors which connect the thermometer to the bridge; the Smith bridge nevertheless requires upkeep and frequent and tedious calibration. This is why a direct current bridge using the principle of the current comparator could advantageously replace the Smith bridge in the near future.

The precision of temperature measurement with the resistance thermometer can be considered as satisfactory: it is better than $0.001 \mathrm{~K}$ in the range $0-100^{\circ} \mathrm{C}$, for example; at elevated temperatures, it is limited to 0.1 or $0.2 \mathrm{~K}$ by the instabilities of the thermocouple. Studies underway, with the aim of reducing this defect, could lead to the abandonment of the thermocouple as an interpolation instrument; the range of temperature left open would be shared between the resistance thermometer which, well constructed, can go beyond $630^{\circ} \mathrm{C}$, and the photoelectric pyrometer which, with modern detectors, becomes practical below $1064{ }^{\circ} \mathrm{C}$.

\section{Pyrometric Study of the Freezing-Point Temperature of Gold}

There is another problem to be considered, that of the accuracy of the values assigned to the defined fixed points: for example, a recent determination of the temperature of the boiling point of water by use of the gas thermometer seems to show that the uncertainty which affects the temperatures is perhaps greater than was estimated in 1968, even for temperatures which were believed to be well known. This is why some work is being pursued in laboratories by diverse methods, notably gas thermometry and pyrometry: the BIPM is participating in these researches and has chosen to study the temperature of the freezing point of gold [3].

The value assigned to this fixed point in the IPTS-68 resulted from several determinations, all made with gas thermometers, whose dispersion is greater than $0.5 \mathrm{~K}$; on the other hand, two studies made later by monochromatic pyrometry furnish values which are lower by several tenths 
of a kelvin. In the face of these disagreements, the BIPM has undertaken a determination, also by a pyrometrie method, but under different conditions of realization.

The principle of the measurement consists in comparing the monochromatic luminances (wavelength in the neighborhood of $1 \mu \mathrm{m}$ ) of two identical radiating cavities. approximating blackbodies, which are observed alternately (fig. 6); the cavities are maintained at $630^{\circ} \mathrm{C}$ and $1064{ }^{\circ} \mathrm{C}$ respectively: their temperatures are measured by two thermocouples which give the temperature values in the IPTS. If at $630^{\circ} \mathrm{C}$ the difference between the true temperature and its value in the IPTS is known, the pyrometric method permits determining the corresponding difference at $1064{ }^{\circ} \mathrm{C}$. By application of the same principle, the study has been extended to the temperature of the freezing point of silver and to several intermediate temperatures between $630{ }^{\circ} \mathrm{C}$ and $1064{ }^{\circ} \mathrm{C}$. The use of an odd number of intermediate temperatures constitutes moreover a useful variant in the study of the freezing point of gold; it permits the comparison of the luminances at $630{ }^{\circ} \mathrm{C}$ and $1064{ }^{\circ} \mathrm{C}$ of the same radiating cavity, which eliminates the effect of possible dissimilarities between the two cavities.

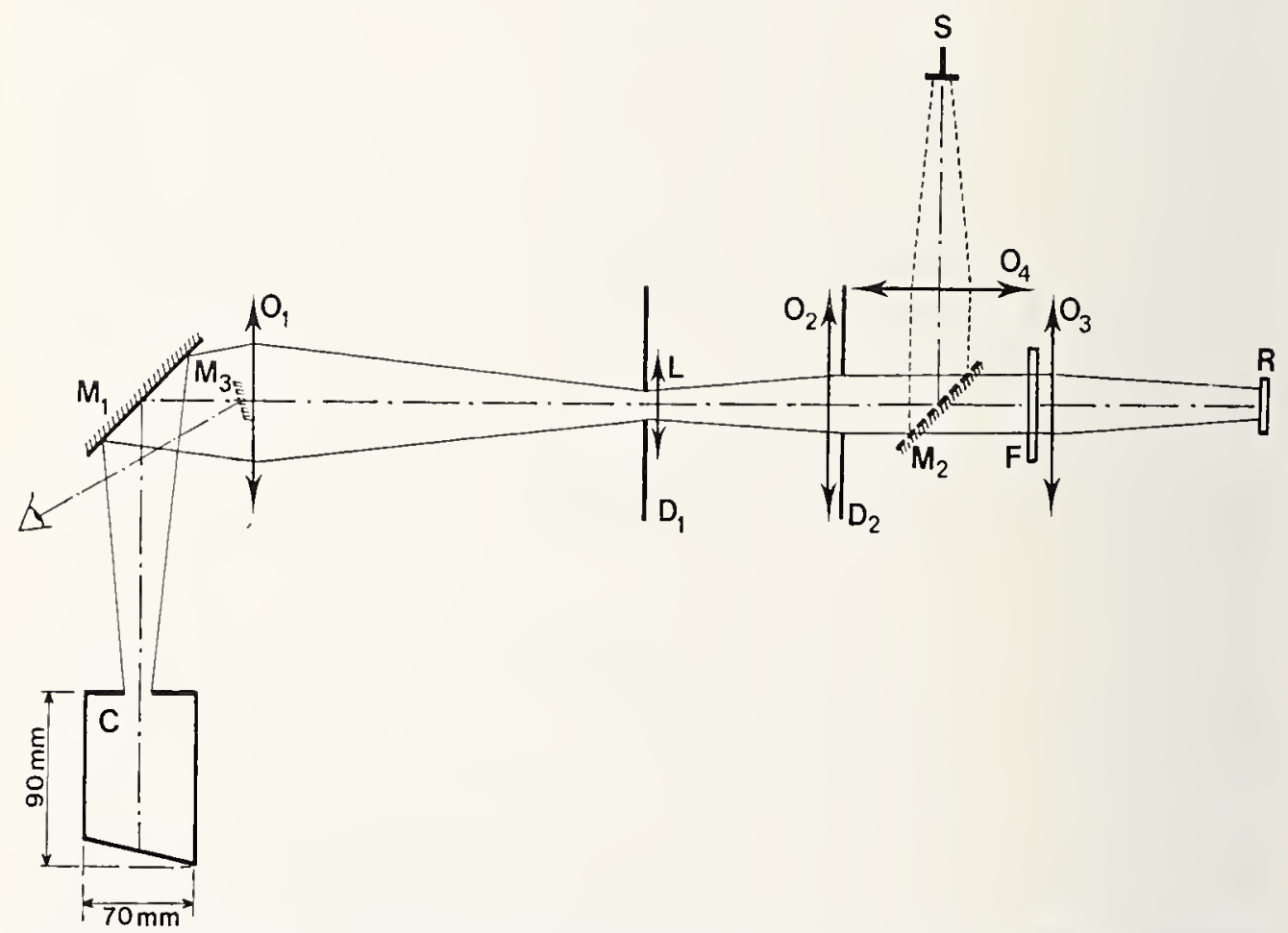

Figure 6. Pyrumetric determination of the freezing point of wold; the optical measuring device. $\mathrm{M}_{1}$. aluminum coated quartz mirror: $\mathrm{O}_{1}, \mathrm{O}_{2}, \mathrm{O}_{3}$. $\mathrm{O}_{4}$ oljectives: L. field lens: $\mathrm{D}_{1}, \mathrm{D}_{2}$ diaphrams; $F$, interference filter: R. detector; $C$, aperture of the blacklondy cavity; $S$, mercury vapor lamp, $\mathrm{N}_{2}$, retractable mirror.

The letector $R$ (photoelectric tube of very good linearity) observes alternately the cavity at $630^{\circ} \mathrm{C}$ and that at $1064{ }^{\circ} \mathrm{C}$ : the centering of the cavity is satisfactory when the green light spot furnished by the ensemble $\mathrm{S}, \mathrm{O}_{4} \cdot \mathrm{II}_{2}$ completely enters the aperture $\mathrm{C}$ of the cavity in question: this adjustment is verified with the aid of $\mathrm{M}_{3 \text { - }}$ 
By this approach, the ratio of luminances, which is of the order of 150 for the direct comparison, is lowered to 12, or evcn less, which facilitates its measurement; on the other hand, there is no longer only one ratio to be determined, but several.

The current work is distinguished from similar determinations in several respects. First. we have obtained an emissivity very close to unity for the radiating cavities (fig. 7), by hollowing out in blocks of pure nickel, cavities having a very high "effective surface/aperture" ratio (above 700); next, we have used techniques perfected in the course of other work to develop ovens of very high stability and very good temperat ure uniformity; finally, we have chosen interference filters to isolate the desired spectral band (half-width from 8 to $15 \mathrm{~nm}$ ).

The difficulties encountered are essentially due to the thermocouples and interference filters; the formcr do not permit temperature verification to better than $0.1 \mathrm{~K}$; the latter are likely to give very weak residual transmission over very broad spectral ranges outside the useful transmission band, and these effects are difficult to evaluate with precision.

The results obtained, in good agreement with those of oilher laboratories, show that the IPTS-68 is not a satisfactory representation of thermodynamic temperatures in the range $630-960^{\circ} \mathrm{C}$; on the other hand, the temperature interval between the freezing points of silver and gold seems

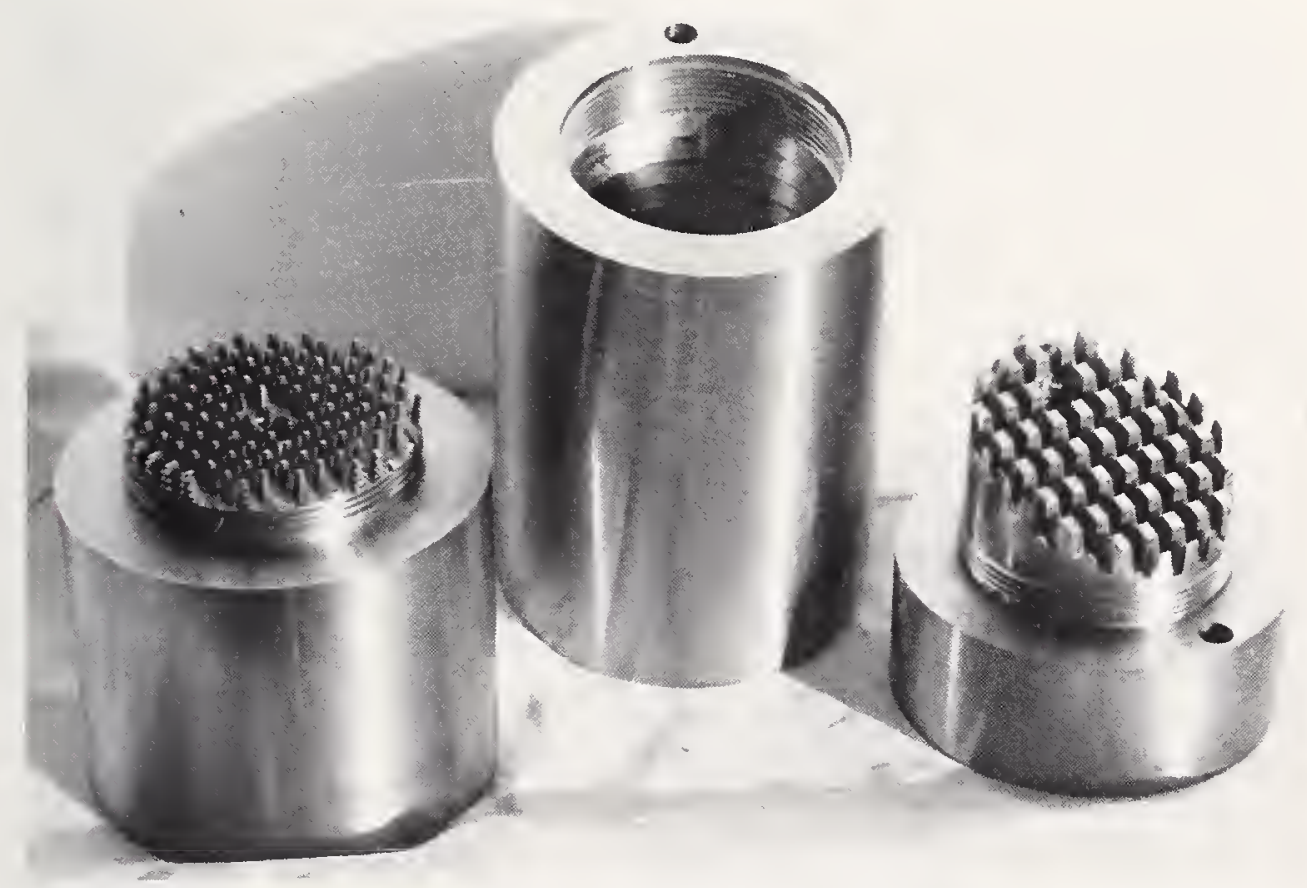

Figure 7. Pyrometric determination of the freezing point of gold: view of the interior of a radiating cavity. From left to right, the cover, the cylinder, and the base. The internal wall of the cavity is grooved and cross-ruled to increase the surface/opening ratio. and the bottom is slightly inclined to diminish the loss of radiation by specular reflection. At the center of the cover, one sees the aperture ( $10 \mathrm{~mm}$ diameter). 
to be correctly evaluated (fig. 8). But the problem is much more general [4] and much work remains to be donc to render the mcasurcments more exact, in particular at very low and very high temperatures.

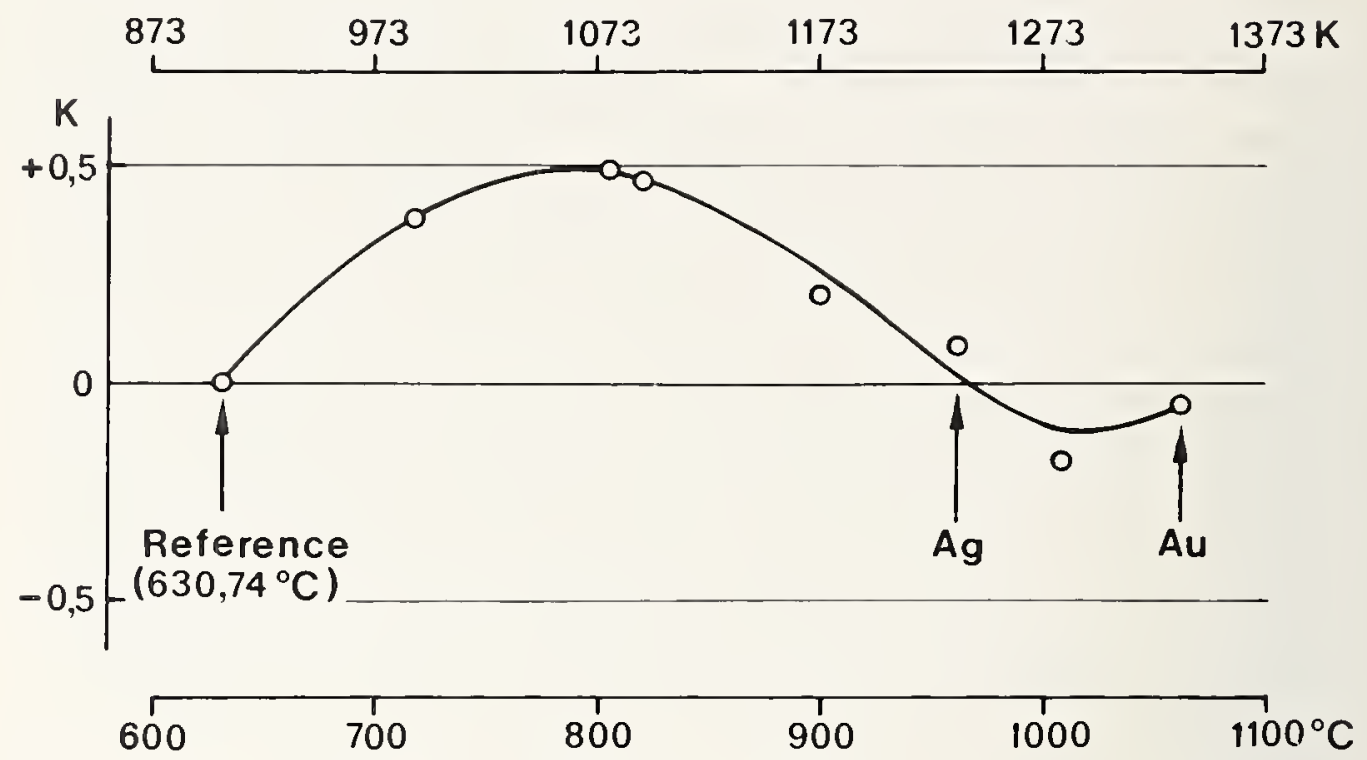

Fiqure 8. Pyrometric determination of the freezing point of oold; results obtained. The curve nives frum 630 to $1064{ }^{\circ} \mathrm{C}$, the difference between the thermodynamic temperature and the temperature in the IPTS- 68 . One accepts by hypothesis that the temperature $630.74{ }^{\circ} \mathrm{C}$ in the IPTS-68 is exact and the $c_{2}$, Planck's second radiation cunstant, has the value 0.014388 $\mathrm{m} \cdot \mathrm{K}$. The precisions of the freezing points of silver and gold are 0.13 and $0.16 \mathrm{~K}$ respectively.

\section{Bibliography}

[1] Guillaume, Ch.-Ed., Etudes thermométriques, Travaux et Ménoires B.1.P.M. 5, 251 pages (1886).

Chappuis, P'., Etudtes sur le thermomètre à gaz et comparaison des thermomètres à mercure avee le thermomètre à gaz, Travaux et Mémoires B.1.P.M.6, 312 pagres (1888).

Chappuis, P., Nouvelles études sur les thermomètres à gaz, Travaux et Mémoires B.I.P.M. 13, 66 pages (1907).

[2] Echelle Internationale Pratique de Température de 1968, Comptes Rendus $13^{e}$ C.G.P.M., 24 pages (1967); Comité Consultatif de Thermométrie, 8th session, pp. AlA24 (1967).

[3] Bonhoure, J., Température du point de eongélation de l'or, Comité Consultatif de Thermométrie, 9th session, pp. T84-T87 (1971).

Bonhoure, J., Etude pyrométrique des températures comprises entre $630{ }^{\circ} \mathrm{C}$ et 1064 ${ }^{\circ} \mathrm{C}$, Comité Consultatif de Thermométrie, 10th session, à paraitre (1974).

[4] Temperature, Its Measurement and Control in Seience and Industry, 2, 444 pages (1955); 3, 2500 pages (1962). Reinhold Publishing Corp., New York; 4, 2383 pages (1973), Instrument Soeiety of America, Pitshurgh. 


\section{CHAPTER VII}

\section{ELECTRICITY}

The BIPM took a very early interest in electrical measurements. As early as 1884, for example, at the request of the International Committee for Weights and Measures, J. R. Benoit constructed on behalf of the French Ministry of Postal and Telegraph Services, 4 prototypes of the legal ohm and about 15 secondary standards. At a time when the ohm was the resistance, at the temperature of melting ice, of a column of mercury of 1 $\mathrm{mm}^{2}$ cross section and $106 \mathrm{~cm}$ length, its construction was essentially a matter of the measurement of length and bore of glass tubing; it was thus natural to entrust it to the specialists of the BIPM who were currently performing this kind of study on mercury thermometers of high precision. Much later, in 1907, it was the BIPM which calibrated and adjusted the tubes destined to realize for France the new ohm defined at Chicago in 1893 (length: $106.3 \mathrm{~cm}$; mass of mercury: $14.4521 \mathrm{~g}$ ).

Although this activity was known and appreciated, it was only in 1921 that the 6th CGPM modified the Convention of the Metre of 1875 to extend the scope of the BIPM to the domain of electric units.

This decision was implemented in 1927 by the 7th CGPM which created for this purpose a Consultative Committee for Electricity (CCE) to "advise the International Committee for Weights and Measures on questions relative to systems of measure and electric standards."

The CCE defined in 1928, in the course of its first session, the functions which needed to be assigned to the BIPM. These were:

- To establish a central secretariat for organizing a systematic exchange of standards and for assuring the synthesis of the results obtained by the national laboratories.

- To establish a laboratory to which physical standards represent ing the results obtained in various countries could be brought for precise comparisons.

- To establish a stock of reference standards and working standards, with the installations necessary for the comparison of other standards with those of the Bureau.

These recommendations, adopted by the CIPM in 1929, define the present mission of the BIPM in the domain of electricity. 


\section{Electric Units}

In 1908, the International Conference of London had noted the concern of physicists with making a very clear distinction between the definitions of the units and their material representations.

At this time, the electric units called "practical" were defined in terms of the CCS electromagnetic units and comprised part of the "Practical system of units"; they were for example: the ohm which had the value $10^{9}$ CGS elcctromagnetic units of resistance, the ampere equal to $10^{-1} \mathrm{CCS}$ electromagnetic unit of current, the volt equal to $10^{8}$ CCS electromagnetic units of potential difference.

On the contrary, the representations of these units constituted another system called "International System," based on two material standards: the international ohm, the resistance offered to an unvarying electric current by a column of mercury of a mass of 14.4521 grams, of a constant cross section and of a length of 106.300 centimetres, at the temperature of melting ice, and the international ampere, an unvarying electric current which, in a solution of silver nitrate in water, deposits silver at the rate of 0.001118 00 gram per second.

After the London Conference, there were then two systems:

- the CCS system, absolute but purely theoretical (with its practical multiples and submultiples: ohm, volt, etc.).

- and the international system permitting the reproduction of the units with the best precision possible at the time, and the assurance of uniformity of electrical measurements throughout the world.

In 1908, the agreement between the two systems was satisfactory but, very rapidly, the progress of electrical metrology made annoying divergences appear. As early as its first session, in November 1928, the CCE therefore proposed to the CIPM to substitute for the international units, an absolute system derived from the CCS units, as soon as it would be possible to "fix with the desired accuracy the ratios between the absolute units and the international units of current, electromotive force, and resistance" [1].

In June 1939, the CCE judged that this accuracy had been realized and recommended the transition to the absolute units; but the world events that occurred several months later did not allow the CIPM to meet before 1946. and it was necessary to wait until that time for the CCE recommendations to be adopted [2].

In 1946, the CIPM fixed the date of 1 January 1948 for putting the absolute electric system of units into effect (in usage since then) and indicated the relation to be used for converting from the international units

\footnotetext{
1 This "International System," designated thus in 1893 by the Congress of Chicago to distinguish it from the absolute CGS System. should not be confused with the International System of Units (SI) adopted by the 11th CGPMI (1960).
} 
to the absolute units. It also gave definitions of the electric units; we recall below those of the ampere, volt, and ohm.

- The ampere is that constant current which, if maintained in two straight parallel conductors of infinite length, of negligible circular cross section, and placed 1 metre apart in vacuum, would produce between these conductors a force equal to $2 \times 10^{-7}$ MKS unit of force [newton] per metre of length.

- The volt is the difference of electric potential between two points of a conducting wire carrying a constant current of 1 ampere, when the power dissipated between these points is equal to 1 watt.

- The ohm is the electric resistance between two points of a conductor when a constant potential difference of 1 volt, applied to these points, produces in the conductor a current of 1 ampere, the conductor not being the seat of any electromotive force. [3].

These decisions of the CIPM were ratified by the 9th CGPM in 1948

\section{Standards}

Although in the International System of Units (SI) the electric base unit is the ampere, the two units whose material representations have always served jointly as the departure point for electrical measurements in all laboratories are the units of resistance and electromotive force; the ohm and the volt are in fact the units which we know how to realize and maintain with the most accuracy.

\section{Resistance Standards}

To be completely defined these standards have four terminals: two current leads and two potential taps (fig. 1). The best quality standards are the standards of nominal $1 \Omega$ value developed by the national laboratories (fig. 2). They are constituted of a metallic wire of an alloy having a low temperature coefficient, for example, manganin (85\% copper, $12 \%$ manganese, $3 \%$ nickel); they should in fact have the least possible sensitivity to variations of the ambient temperature and to the heating produced by the passage of the measuring current. This wire, of 1.2 to $1.5 \mathrm{~mm}$ diameter, is wound in the form of a double helix (to reduce the self-inductance) and carefully annealed to assure the stability of its resistance; it is placed inside a hermetically sealed container filled with pure mineral oil or an inert gas. 
The standards of good quality have a temperature coefficient between 5 and $10 \times 10^{-6} / \mathrm{K}$ in the neighborhood of $20^{\circ} \mathrm{C}$; their resistance may change slowly with time, but this change does not exceed $1 \times 10^{-7}$ per year for the best standards.

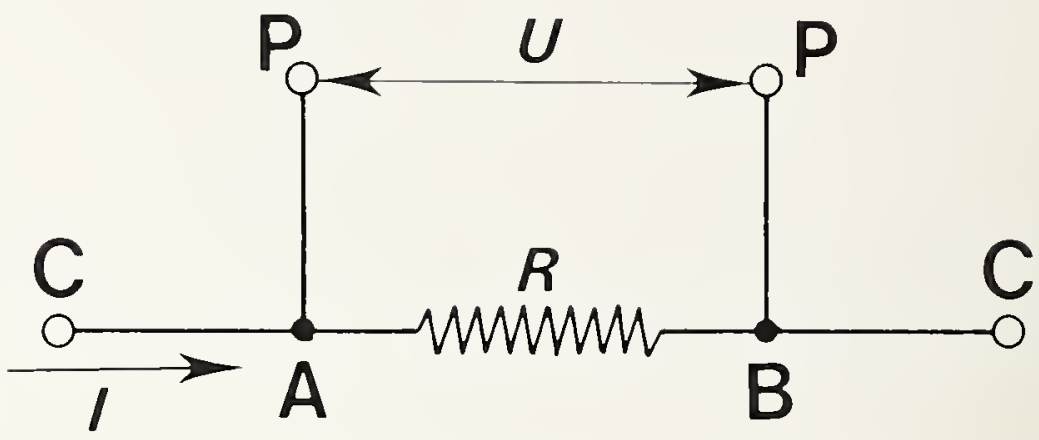

Figure 1. Diagram showing the principle of a standard resistor: $R=U / I$, C. terminats for the current feed: l', potential taps. To measure such a resistor it is necessary to use an arrangement which athows rhmmation of the effect of the resistances in arms AC. BC. AP. and BP

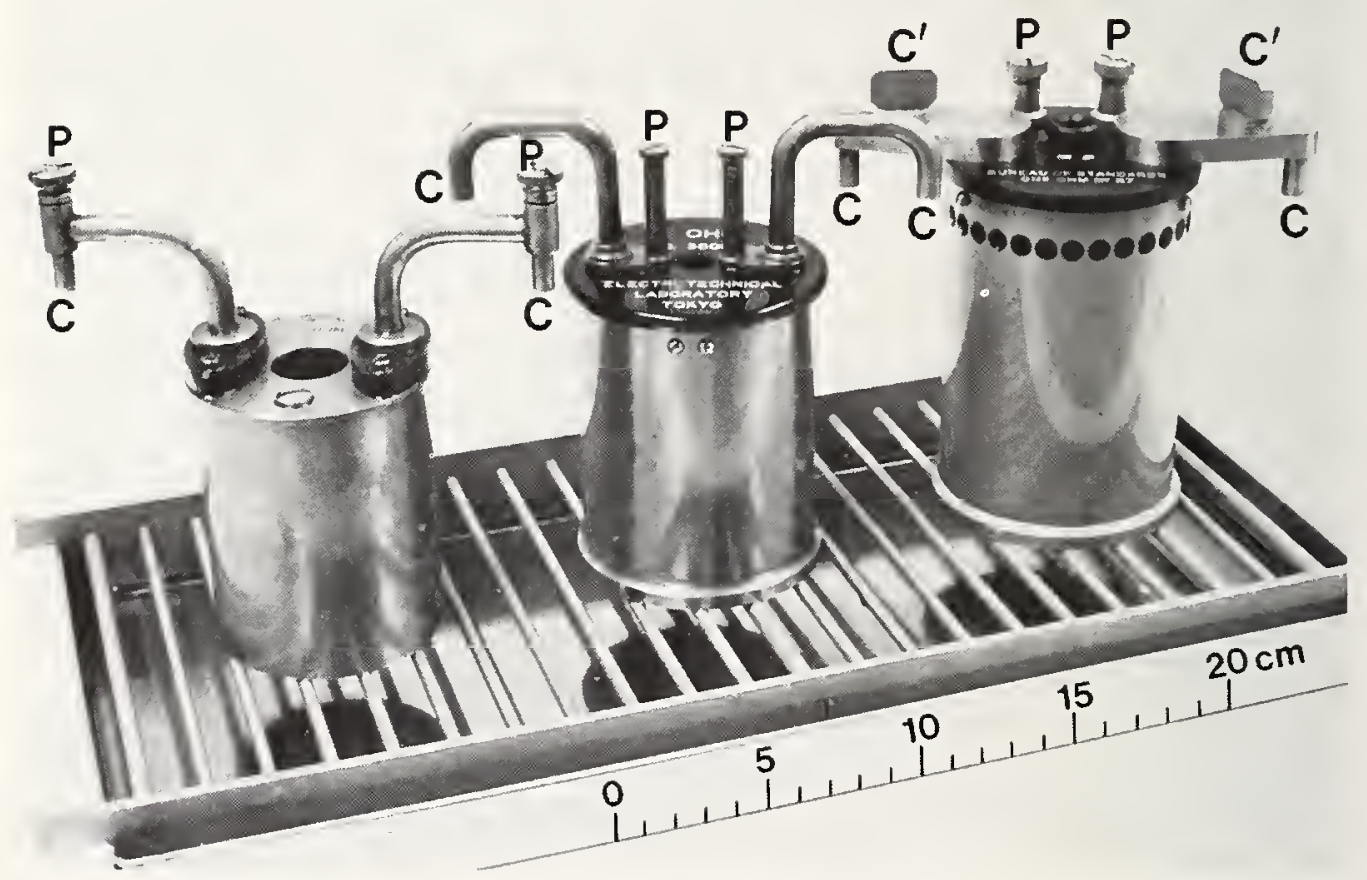

Finure 2. Resistance stantards of $1 \Omega$ developed by national laboratories. To insert a standard into the measuring circuit. terminals $C$ are set in cups filled with mercury (for the third standard (1) the right, one can also clamp wires under the terminals $C^{\prime}$ ). Terminals Perve in the measurement of the rultage drop which results fom the passage of current through the resistor. 
The volt is "maintained" by means of cells - or elements - of the saturated cadmium sulfate type (developed by Weston in 1893), whose electromotive force at $20^{\circ} \mathrm{C}$ is of the order of $1.01860 \mathrm{~V}$. These cells belong to the category of elements called "reversible," each of the electrodes being immersed in an aqueous solution of a salt of the metal which constitutes the electrode (fig. 3).

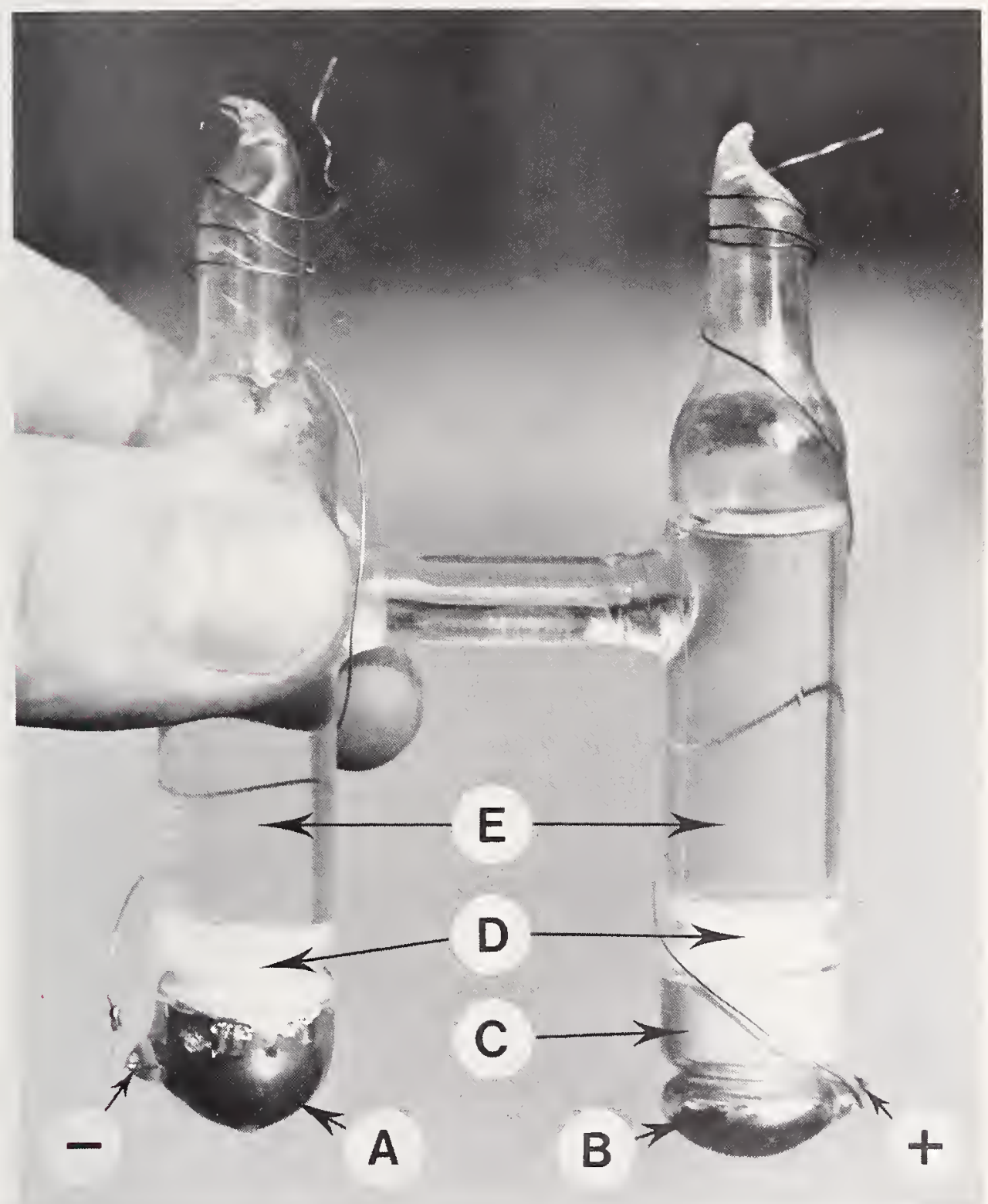

Figure 3. Cadmium-sulfate standard cell. Negative limb: A, cadmium amalgam in 2 phases: Pusitive limb: B, pure mercury: C. mercurous sulfate $\mathrm{Hg}_{2} \mathrm{SO}_{4}$ : E. saturated solution of cadmium sulfate: $\mathrm{D}$, crystals of cadmium sulfate $\mathrm{CdSO}+8 / 3 \mathrm{H}_{2} \mathrm{O}$. 
The Weston elcment is a fragile standard: it is sensitive to shock. vibration, and light; a reversal is fatal to it. But it is especially sensitive to temperature variations which render its use ticklish.

The electromotive force of a saturated Weston elcment decreases about $40 \mu \mathrm{V}$ when its temperature increases by 1 degree, in the neighborhood of $20^{\circ} \mathrm{C}$; furthermore it varies by $330 \mu \mathrm{V}$ per degree of temperature difference between the two limbs. For the electromotive force of a standard ccll to be defined to about $10^{-7} \mathrm{~V}$, it is thus neccssary that its temperature be known in absolute valuc to about $2.5 \mathrm{mK}$ and that the temperature of its two limbs be cqual within about $0.3 \mathrm{mK}$.

The Weston element also has a serious defect as a standard: not only does its electromotive forcc require several weeks to stabilize after a temperature change, but in general it does not recover exactly its initial value when the initial temperature is reestablished. Only a few very well equipped laboratories can thus achieve a high stability: a microvolt per year or even several tenths of a microvolt per year for the most successful elements.

\section{Installations of the BIPM}

The study of typical standards for the ohm and the volt comprises the basic experimental activity of the BIPM. To carry out this study and to maintain the standards under the best conditions, the BIPM has put various installations into more or less perfect running order.

\section{Kelvin Double Bridge}

A Kelvin double bridge (figs. $4 \mathrm{a}$ and $4 \mathrm{~b}$ ) is used for determining the difference between two resistance standards; this instrument in fact, allows eliminating the influence of contact resistance, of current leads, and of the potential taps of the measured standards.

The standards are compared in turn and repeatedly to a "tare resistance" whose value is adjusted by means of a shunt to obtain bridge balance (null current in the detector).

The standards are kept in a bath of mineral oil. During the measurements this oil is kept in motion by an impeller, to remove the energy dissipated in the standards and in the tare by the measuring current $(0.1 \mathrm{~A})$.

The comparisons are carried out at ambient temperature $(20 \pm 0.05)$ ${ }^{\circ} \mathrm{C}$; the exact temperature of each standard is registered to a few thousandths of a degree by means of mercury thermometers calibrated at BIPM. To eliminate the effect of possible parasitic electromotive force, bridge balances are obtained for the two directions of current.

The installation at the BIPM permits determination of the difference between two $1-\Omega$ standards to about $1 \times 10^{-7} \Omega$. 


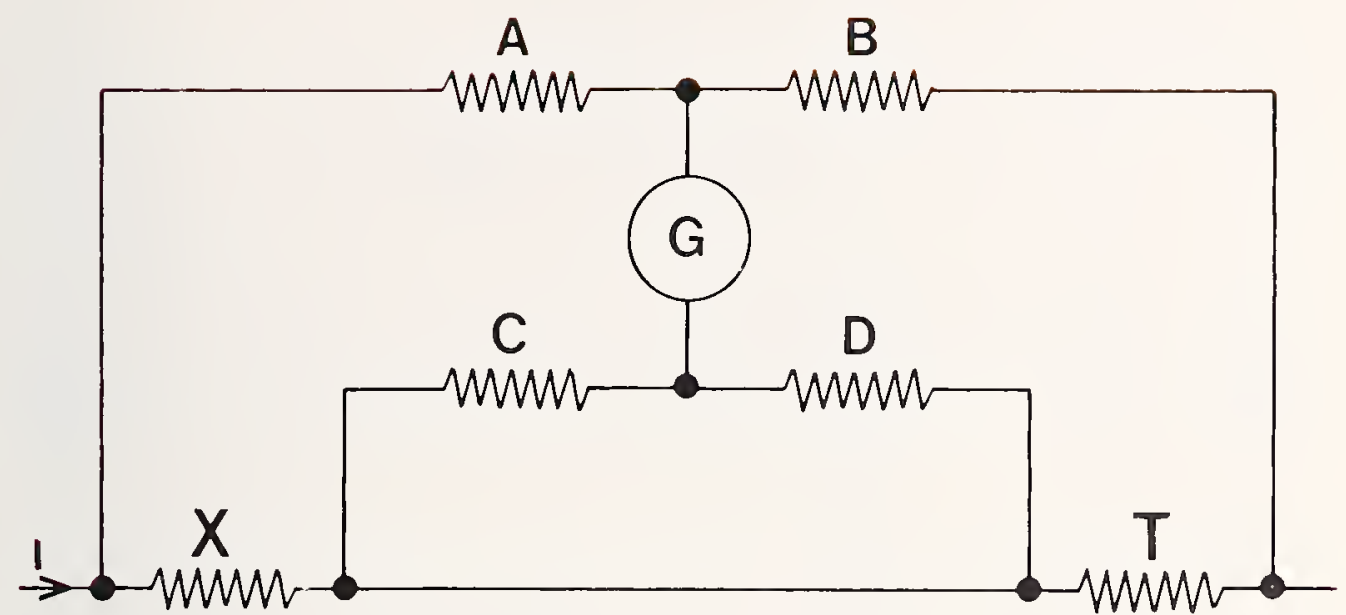

Figure 4a. Diagram showing the principle of the Kelvin double bridge. When there is no current through the detector G, one has the relation:

$$
X / T=A / B=C / D
$$

$X$. standard to he compared: T, tare resistance. 


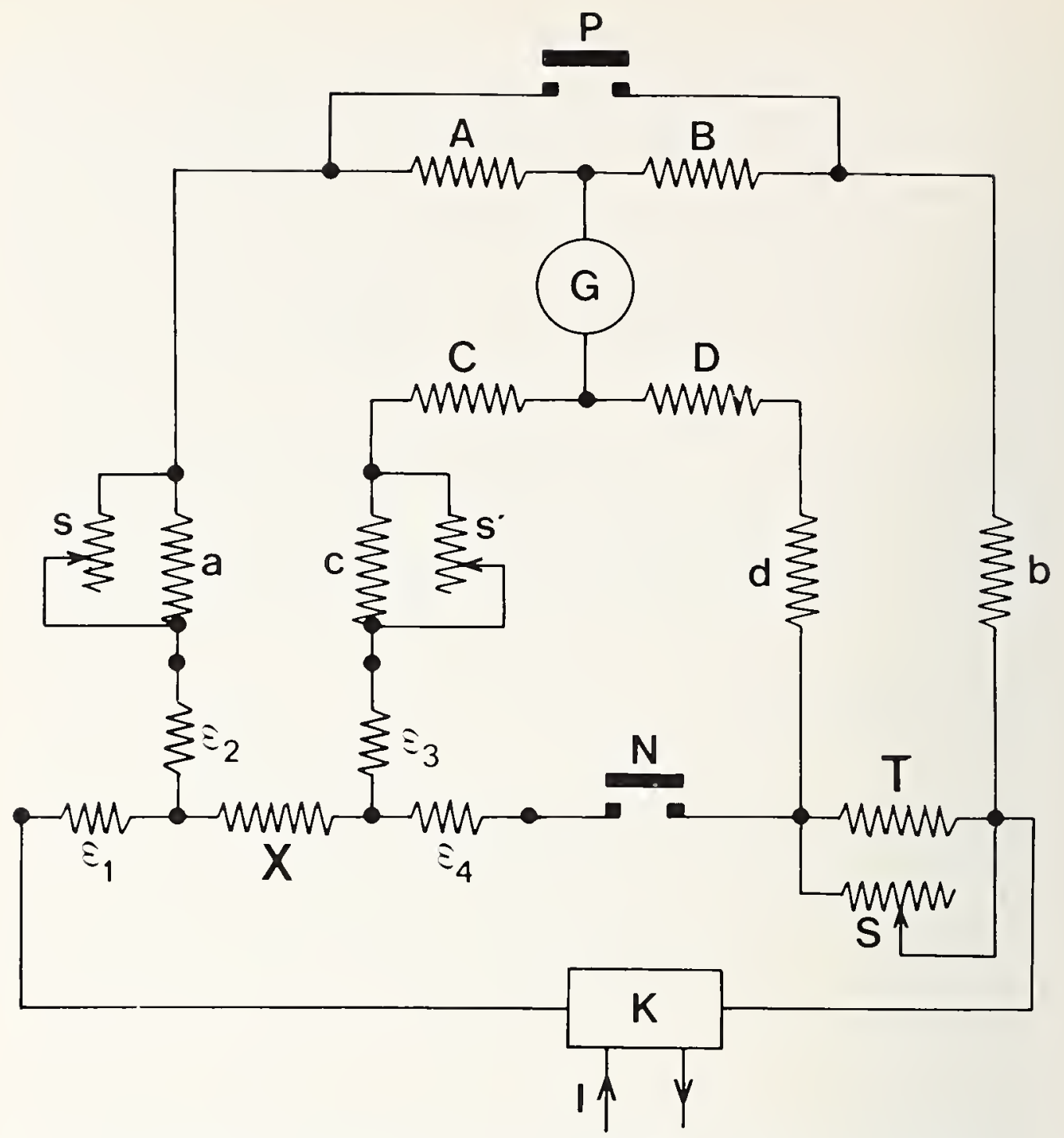

Figure $4 \mathrm{~b}$. Diagram of the double briftge used at the BIPM. X. standard io be compared: T. tare resistance (1.007 $\Omega$ ): S, 6-decade resistance box (resolution: 0.1 $\Omega$ ): A. B. C. D principal bridge resistances (nominal value: $1000 \Omega$ ): $P$ ' and $N$. switches: $K$. current reversing switch: $\epsilon_{1}, \epsilon_{2} . \epsilon_{3} . \epsilon_{4}$. resistances of the arms of standard $X$ and of the comnections (the influence of these parasitic resistances is efiminated by establishing auxiliary batances. the first with $P$ and $N$ closed with adjustment of $s$ across the terminals of a, the second with $P$ and $N$ open and with adjustment of $s^{\prime}$ across the terminats of $\mathrm{c}$. The principal doubte bridge corresponds to $\mathrm{P}$ open and $\mathrm{N}$ closed; it is balanced with adjustment of $\mathrm{S}$. If $\mathrm{S}_{1}$ and $\mathrm{S}_{2}$ are respectively the values needed for $S$ to obtain bridge balance with two standards $X_{1}$ and $X_{2}$ the difference between these two standards is given by:

$$
X_{1}-X_{2}=(A / B)\left\{\frac{T S_{1}}{T+S_{1}}-\frac{T S_{2}}{T+S_{2}}\right\}
$$

If the ratio $A / B$ of the arms of the principal bridge. nominally equal to 1 . is in fact equat to $(1+\delta)$. the difference $\left(X_{1}-X_{2}\right)$ is determined with a relative error equal to $\delta$. By interchanging the arms $A$ and $B$ in the middle of the comparison of the two standards $X_{1}-$ $X_{2}$, the relative error of the difference is retuced $t, \delta^{2} / 2$. 
To determine the difference of electromotive force between two cells, they are connected in opposition and the resulting electromotive force measured by means of a potentiometer. The voltages to be measured are thus always small (at a maximum, several hundred microvolts) and relatively easy to determine with precision. The device used at the BIPM essentially comprises two potentiometers connected in series (fic. 5); the first, A, serves to regulate the potentiometer current $\left(10^{-4} \mathrm{~A}\right)$, the second, B, to measure the difference between the electromotive forces of the two cells to be compared.

For a null detector, we use a galvanometer amplifier whose principal advantage is to avoid discharging the cells during measurement.

The BIPM potentiometer allows determination of the difference between two cells to a few tens of nanovolts.

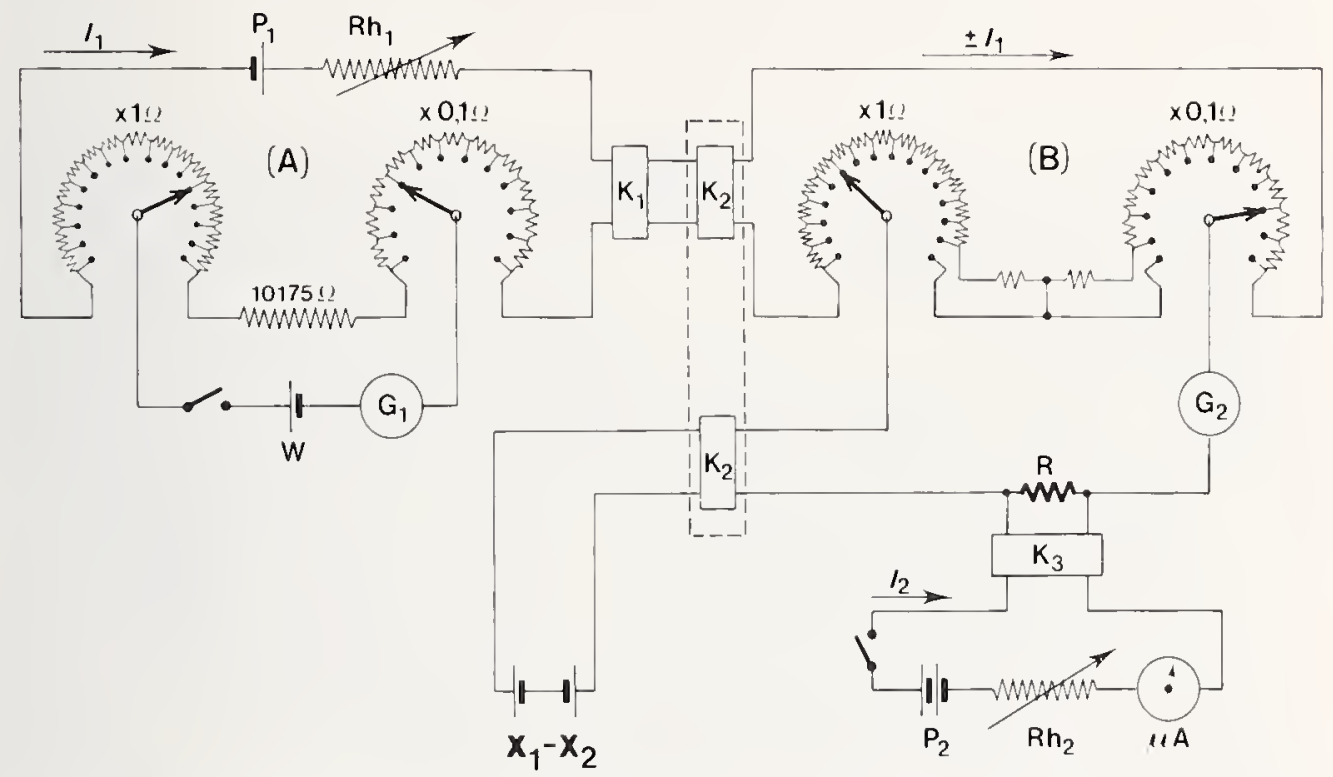

Figure 5. Diagram of potentiometer used to compare two standard cells. $X_{1}$ and $X_{2}$, cells to be compared, connected in opposition; $K_{1}$, reversing switch to give the appropriate polarity $t$, the potential difference to be balanced against $\left(X_{1}-X_{2}\right): \mathrm{K}_{2}$. double reversing switch for simultaneously reversing the sense of the potentiometer current and the polarity of the measured voltage $\left(X_{1}-X_{2}\right)$, for eliminating the effect of possible parasitic electromotive forces in the circuit of the balance detector $\mathrm{G}_{2}$. The potentiometer current $I$, is furnished by a battery of dry cells $P$ and is not interupted. Before each measurement its value is adjusted to $1 \times 10^{-4} \mathrm{~A}$; for this, the potentiometer $(\mathrm{A})$ is set to the known value of electromotive force of a standard cell $\mathrm{W}$ and the rheostat $R h_{1}$ is adjusted for a null on the calvanometer $G_{1}$. When $I_{1}=10^{-4} \mathrm{~A}$. the terminals of the potentiometer (B) supply a potential difference which is adjustable from 0 to $1600 \mu \mathrm{V}$ in steps of $10 \mu \mathrm{V}$. To establish the balance inull current in the detector $\left.C_{2}\right)$, we add algebraically $t_{0}\left(X_{1}-X_{2}\right)$ the potential (lifference created at the terminals of a $l-\Omega$ resistor $\mathrm{R}$ by the passage of a current $I_{2}$ measured by means of the microammeter $\mu \mathrm{A}$. 


\section{Oil Bath for Storage and Measurement of Cells}

The BIPM stores and measures its reference cells in a tank filled with mineral oil (fig. 6). It is also in this tank, which can hold 120 cells, that are immersed the transport cells which participate in the international com. parisons and the cells which the BIPM receives for calibration.

The heat capacity of the oil reduces the temperature fluctuations. It permits obtaining, as quickly as possible, thermal equilibrium of the cells which are sent to the BIPM. During measurement, the rotation of an impeller assures a radial circulation of the oil whose temperature uniformity is thus obtained to better than $5 \times 10^{-4} \mathrm{~K}$ throughout the bath.

In the course of a measurement, which lasts around a half-hour, the temperature rise produced by the agitation of the oil is of the order of $1.5 \mathrm{mK}$.

This bath is not temperature regulated but it is placed in a room kept at $(20 \pm 0.05){ }^{\circ} \mathrm{C}$. Its temperature is determined by means of one or several platinum resistance thermomcters. It is closed by a cover of opaque Plexiglas.

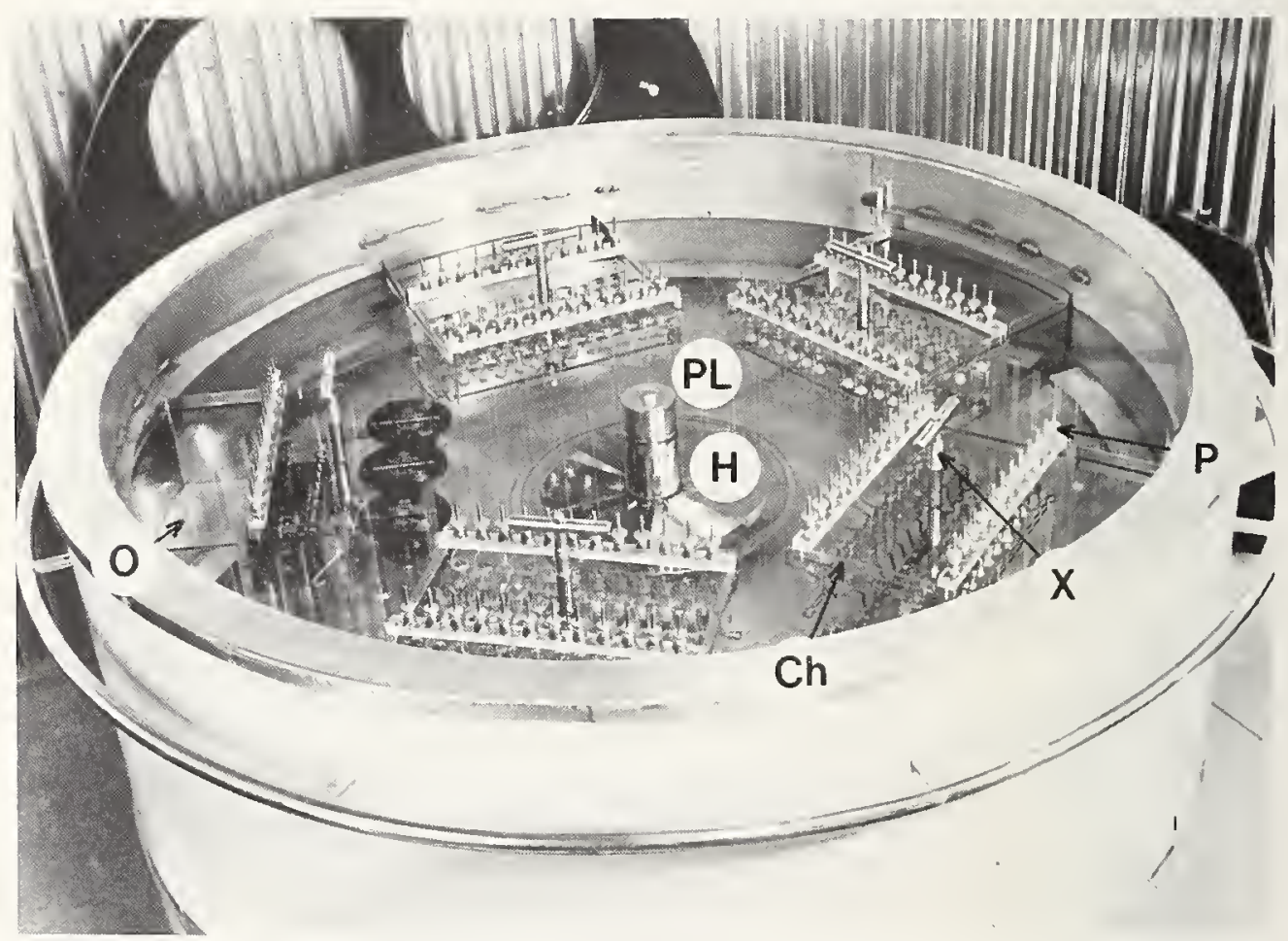

Figure 6. Mineral-oil bath in which the standard cells are stored and measured. Ch. rack supporting the standard cells (each rach can rotate a half-turn about its vertical axis $X$ ); $P$, positive tcrminal (the ncgative terminals are connected to each othcr): PL. Plexiglas partition separating the tank into two compartments; H, impcller assuring circulating of the oil, which passes from the upper compartment to the lower compartment, comes back up through the peripheral orifices 0 , and is then channeled toward the center of the tank by the lateral faces of the racks. 
The racks which carry the cells are mounted on supports which can turn about a vertical axis. By rotation of a half turn the cells placed at the periphery are carried to near the center (and conversely) and the positions of the limbs of each cell relative to the flow of oil are interclianged. The effects of possible temperature gradients are thus eliminated.

\section{Temperature-Regulated Air Enclosure}

To maintain the cells at a temperature still more constant and more uniform than in the oil bath, the BIPM has constructed a temperature-regulated air enclosure whose thermal behavior is being studied. This enclosure comprises cubes, some of thin duralumin walls, others of thermally insulat ing walls of expanded polyst yrene, stacked one within the other (fig. 7). The innermost cube which contains the cells (distributed in 16 massive duralumin blocks) is of copper with thick watls to have a large heat capacity.

This enclosure is placed in the same room, controlled at $20^{\circ} \mathrm{C}$, as is the oil bath; it is maintained at about $21{ }^{\circ} \mathrm{C}$ by electric heating of $10 \mathrm{~W}$ uniformly distributed on the six faces of the next to the outer metallic cube. Its temperature is regulated by a mercury thermometer with a contact and is measured by means of two $100-\Omega$ platinum probes, a thermistor bridge, and two copper-constantan thermocouples lodged in the middle partition of the interior chamber.

To reduce possible heat exchange between the cells and the exterior, the conductors which lead to the cell terminals are teflon insulated multistrand copper cables of very small cross section $\left(0.014 \mathrm{~mm}^{2}\right)$, very long (about $4 \mathrm{~m}$ ), and wound in a spiral.

\section{Periodic International Comparisons}

To assign values to the resistance standards and standard cells which constitute their reference groups, the principal national laboratories carry out absolute determinations. These are electromechanical experiments which relate the electric units to the units of length, mass, and time in conformity with the definition of the ampere. These are difficult measurements. long and costly, which must, it is well understood, be repeated periodically; their precision is, for most of the electric units, inferior to the stability of physical standards which represent these units, and to the com parison measurements between these standards. For these reasons, and for reasons of continuity, the national laboratories do not modify the values of their reference standards each time they carry out absolute determinations. They do this only exceptionally. practically in the three following cases: 


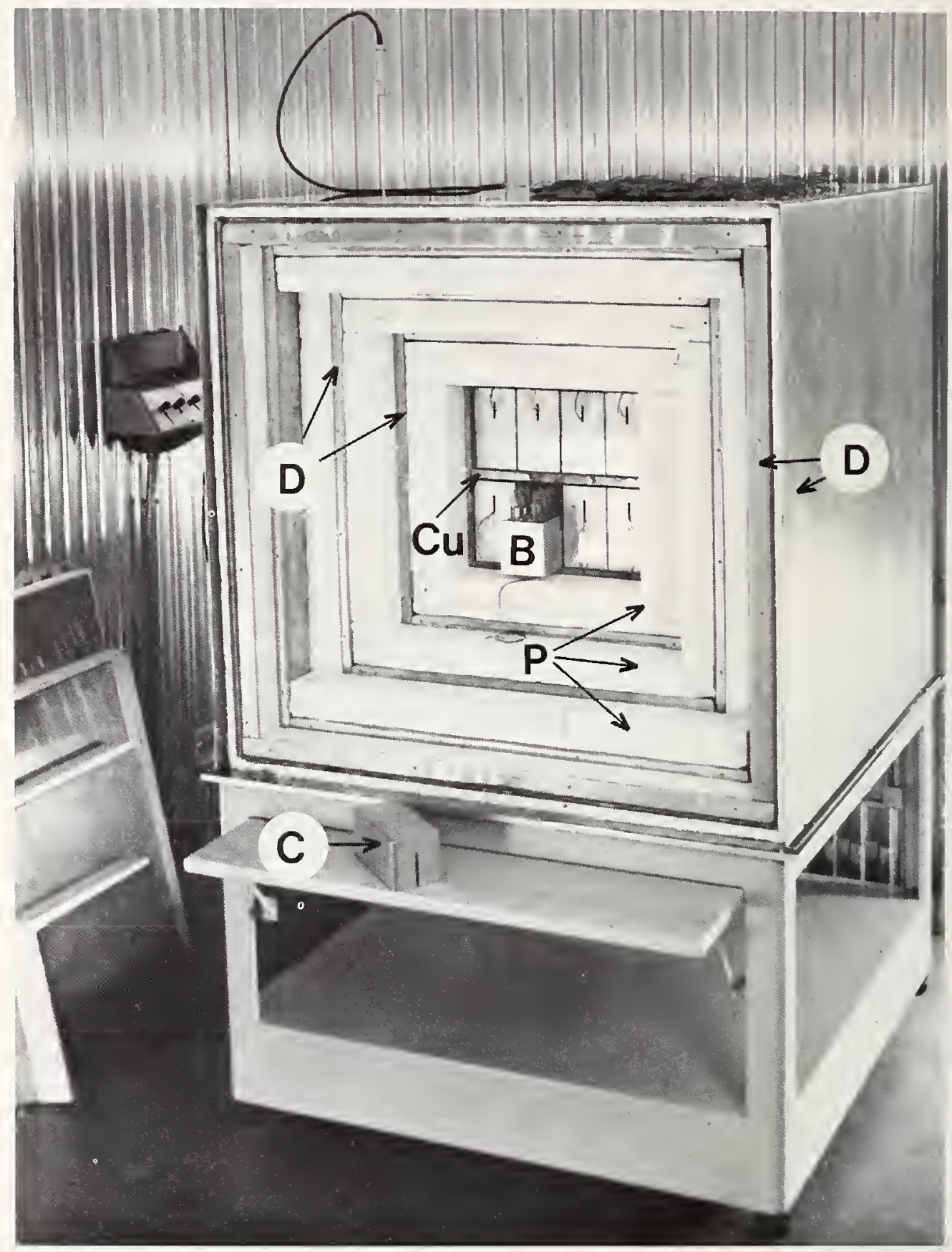

Figure 7. Tempcraturc-regulated air enclosurc for storing cells. Cur. interior cube of copper with its middle pratition enclosing the platinum probes, thermistors. and thermocouple junction which permit knowing the temperature of the cells: D, duralumin cubes: P. expanded polystyrene: B, duralumin blocks which can each hold six cells: C, cover for a block. 
- When the difference between the result of the new detcrmination and the value previously assigned exceeds the uncertainty of the measurements, and when some particular reasons can explain an abnormal change in the material standards (bad storage conditions, for example).

- When the progress of metrology permits improving the precision of the absolute determinations (this has been the case for several years for the farad and the ohm),

- To improve international uniformity (as was the case for the ohm and the volt the lst of January 1969).

The reference groups maintained in various national laboratories comprise only approximate representations of the units (ohm and volt).

One of the essential tasks of the BIPM consists in determining the spread among these representations; it is the object of the international measurements in the world. These comparisons also permit evaluation of the effective precision obtained in the absolute determinations and can lead to the discovery of possible systematic errors.

To participate in these periodic comparisons (biennial from 1932 to 1957, then triennial since 1957), all the laboratories invited by the CIPM simultan eously submit several standards of resistance (of $1 \Omega$ ) and of electromotive force (Weston cells) to BIPM. The transportation of Weston cells requires elaborate precautions; laboratories most often utilize Cardan suspensions to keep them vertical (fig. 8) and transportable temperatureregulated enclosures to avoid all thermal shock.

With the development of high-precision metrology throughout the world, the number of participants in the international comparisons continues to grow. In 1932, the first comparisons had assembled at the BIPM 20 standard cells and $81-\Omega$ resistors provided by 4 laboratories.

In 1973, 11 laboratories sent to the BIPM 59 bare cells (in 10 groups), 37 cells at $30^{\circ} \mathrm{C}$ (in 9 temperature-regulated enclosures), 36 1- $\Omega$ resistors, and for the first time, 15 resistors of $10^{4} \Omega$. The duration of the measurements has a parallel growth; those of 1973 lasted about 4 months.

Given the large number of standards, it is not possible, also not useful, to compare them two by two in all possible combinations. We therefore adopted some simplified comparison schemes; an example of comparison of resistance standards is reproduced in figure 9.

For resistors as for cells, each measurement is made twice, at times which are symmetrical relative to the central date of the comparison, to eliminate in the first approximation the possible drifts of the standards. 


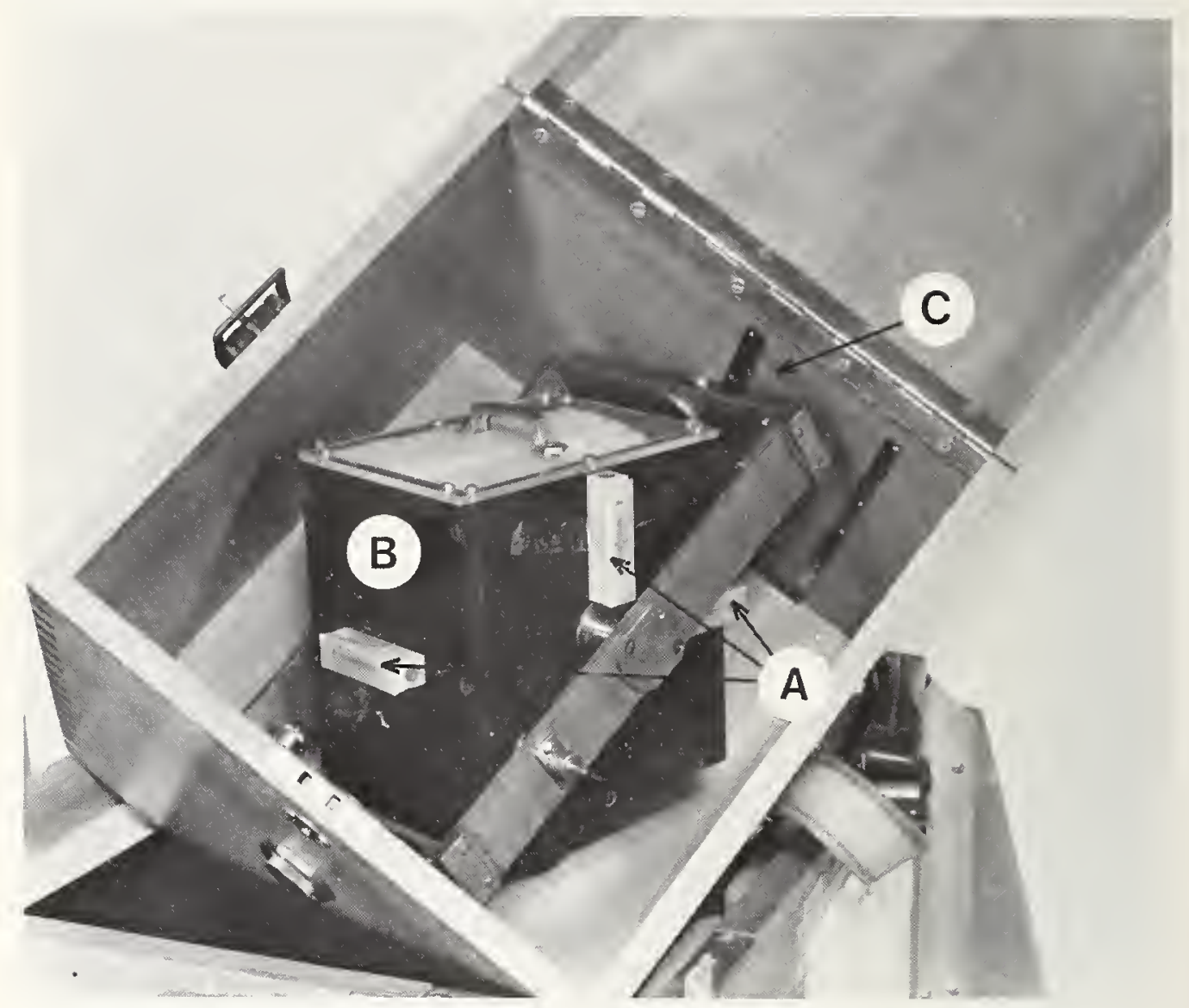

Fiqure 8. Shipping case with Cardan suspension for the transportation of cells by freight. The box $\mathrm{B}$ which holds the cells remains vertical during transport. A, accelerometers recording maximum acceleration (shuch. in particular): C, recorder for uscillations (a second recorder is invisible in this photograph). 


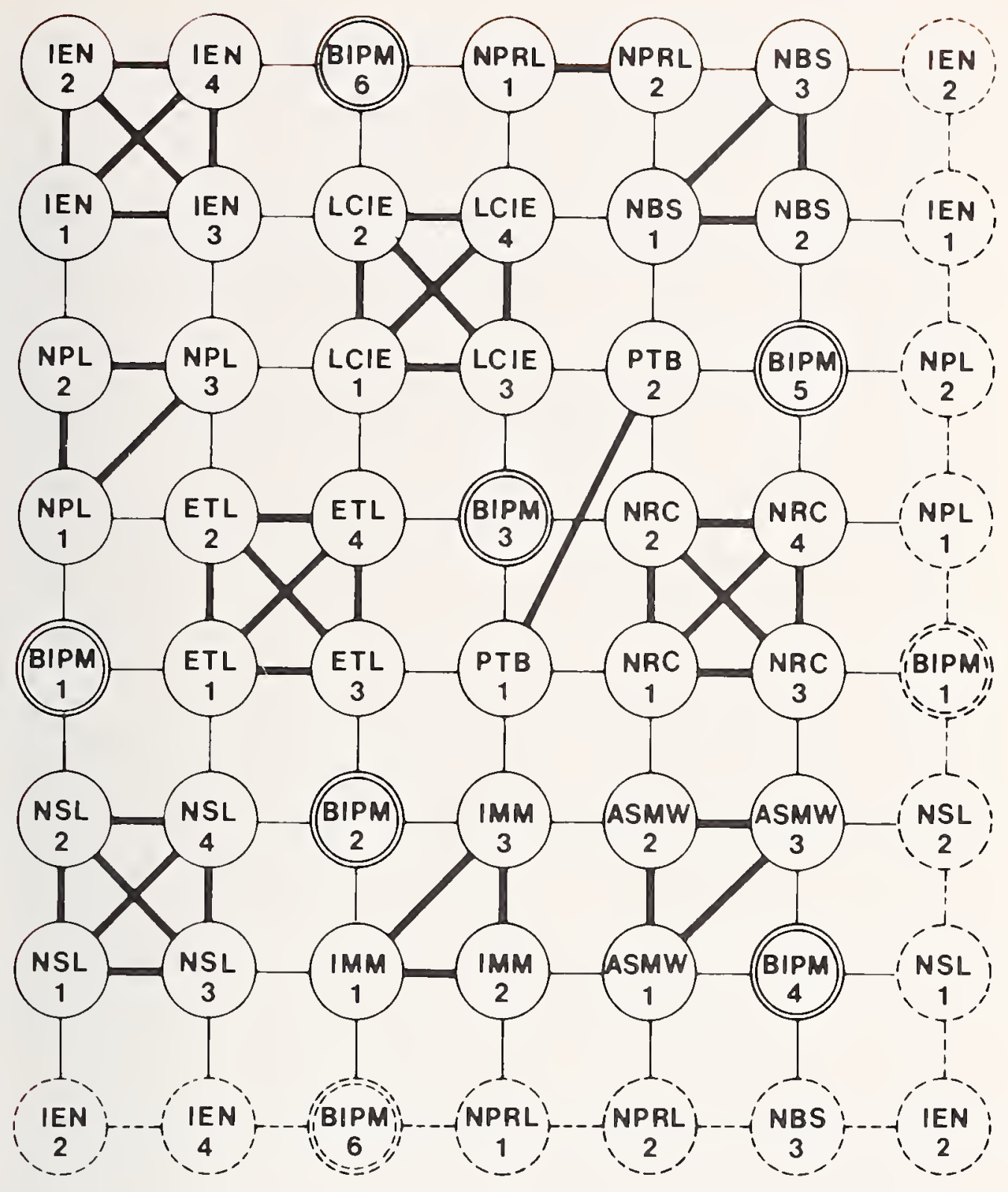

Figure 9. Example of the comparisun scheme for $1-\Omega$ resistancr standards. For comprehension of the scheme, the first column has been repeated on the right in bruken lines, and the first row repeated at the bottom. The standards of the BIPM are identified loy double circles. The standards of a given laboratory are compared amono themselves in all possible combinations (comparisons indicated by heavy lines). This scheme, used in 1973 to compare 42 standards, needed the execution of 198 comparisons $(99$ comparisons "going" and 99 "returning"). 
The results of measurements carried out at the BIPM express the value of the transport standards of national laboratories in terms of the representations of the ohm and the volt maintained by the reference groups of the BIPM.

Before their trip to the BIPM and after their return, the transport standards are carefully calibrated in their laboratory of origin; thus it is verified that they have not been subject to any damage by reason of their transport, and their probable values are calculated for the central date of the measurements made at the BIPM.

In comparing the two values attributed to the transport standards for the same date, we deduce the divergences between the representations of the units maintained by the national laboratories and those which are maintained by the BIPM.

Figures 10 and 11 show the relative changes, since 1950, of the representations of the units of resistance and of electromotive force maintained by the national laboratories.

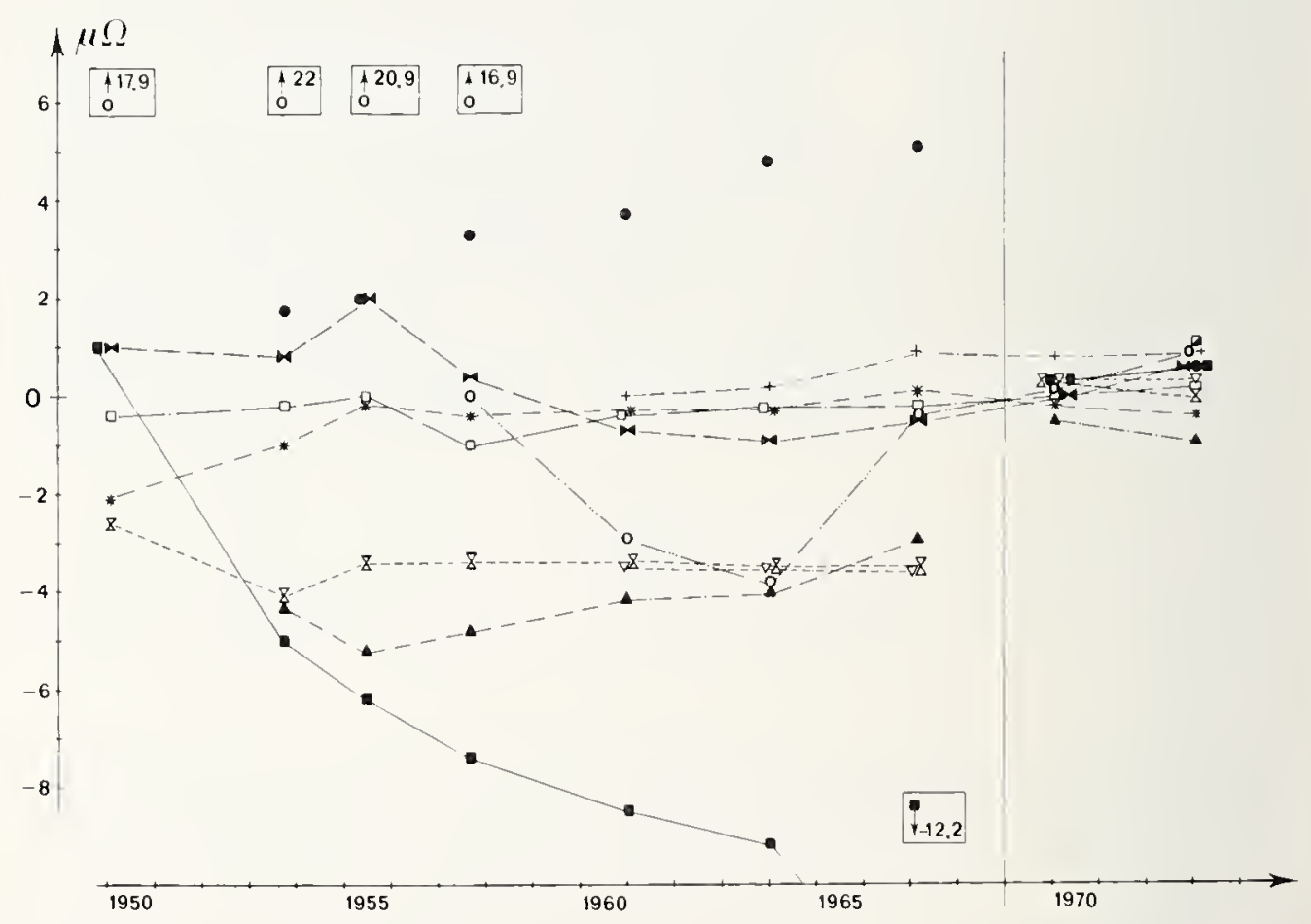

Figure 10. Results of the periodic comparisons of national resistance standards, from 1950 to 1973 (difference between the reference values maintained by the national lahoratories and that of the BIPM). In 1957, the ASMW reduced the value of its national reference by 16.9 $\mu \Omega$ to adjust it to that of the BIPM. To achieve a better international uniformity, the PTB, the NSL, the NRC, the LCIE, and NPL adjusted the values of their rational references to that of the BIPM on the lst of January 1969. П NPRL;OASMW:OPIB: $\square$ NSL; $\triangle N R C$;

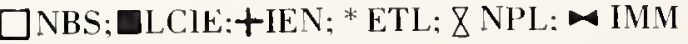




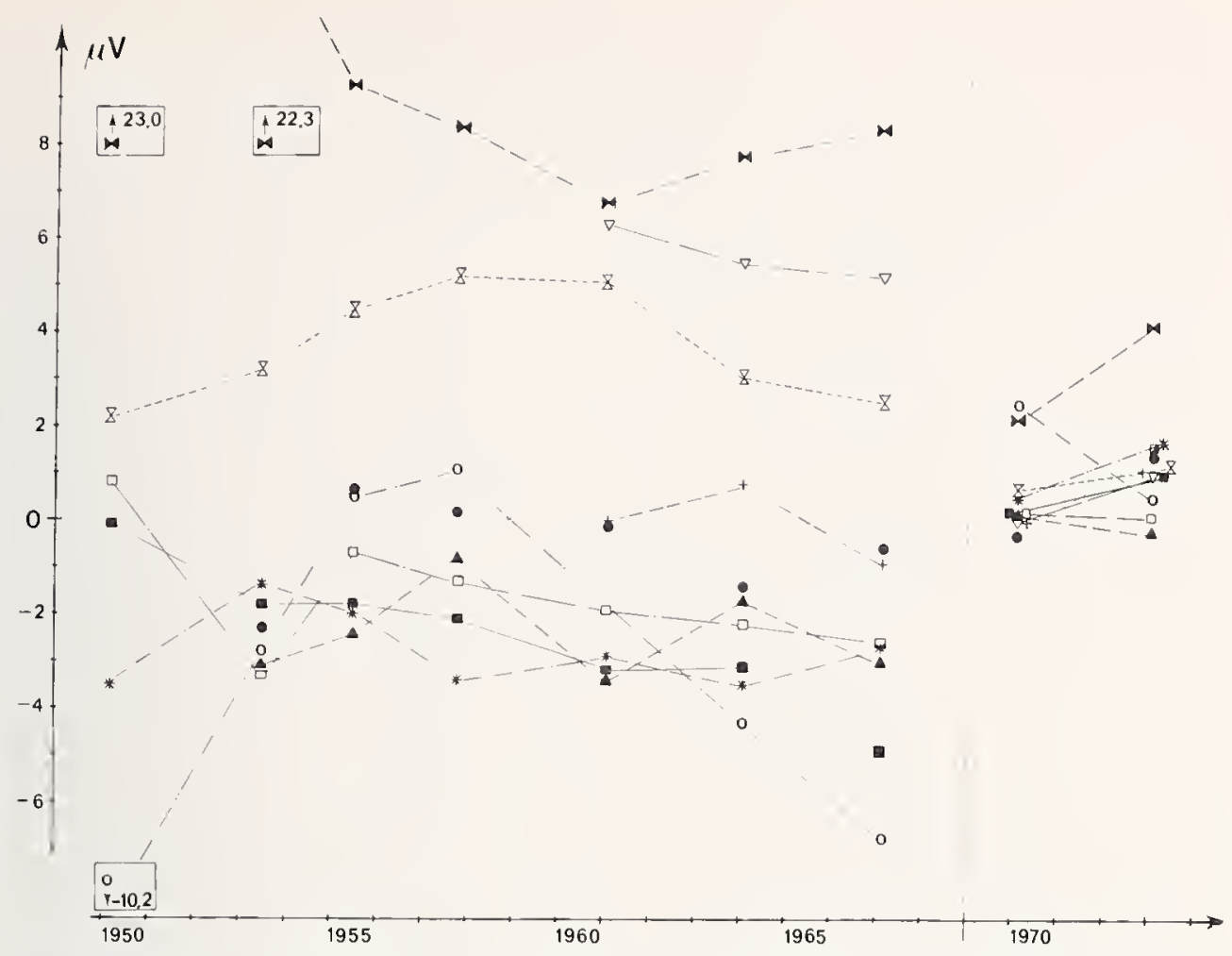

Figure 11. Results of the periodic comparisons of national standards of electromotive force, from 1950 to 1973 (difference between the reference values maintained by the national laboratories and that of the BIPM). On January 1, 1969, the reference maintained by the BIPM was reduced by $11 \times 10^{-6}$ to put it into closer agreement with the volt. On the same date all the national laboratories, except the ASMW, also corrected their national references to make them coincide with the new reference of the BIPM. For the identifica. tion of the signs, see figure 10 .

\section{Maintenance of the Ohm and the Volt by the BIPM}

As do also all the national laboratories, the BIPM maintains the ohm and the volt by means of $1-\Omega$ resistance standards and saturated cadmium sulfate cells. The majority of these standards (especially resistors) were fabricated by various national laboratories and deposited at the BIPM in accordance with the recommendation of the CCE (1928) approved by the CIPM. Currently, $61-\Omega$ standards and 43 cells constitute the reference groups of the BIPM. It is by comparison with these references that the BIPM determines the value of standards which are submitted to it for study.

What do the references maintained at the BIPM represent?

Until 1935, these references, identified by the symbols $\Omega_{\mathrm{m}}$ and $V_{\mathrm{m}}$, were the averages of material representations of the ohm and the volt in use in the national laboratories which had participated in the most recent periodic international comparisons. They could consequently vary on the occasion of each comparison; it sufficed for this that a new laboratory came 


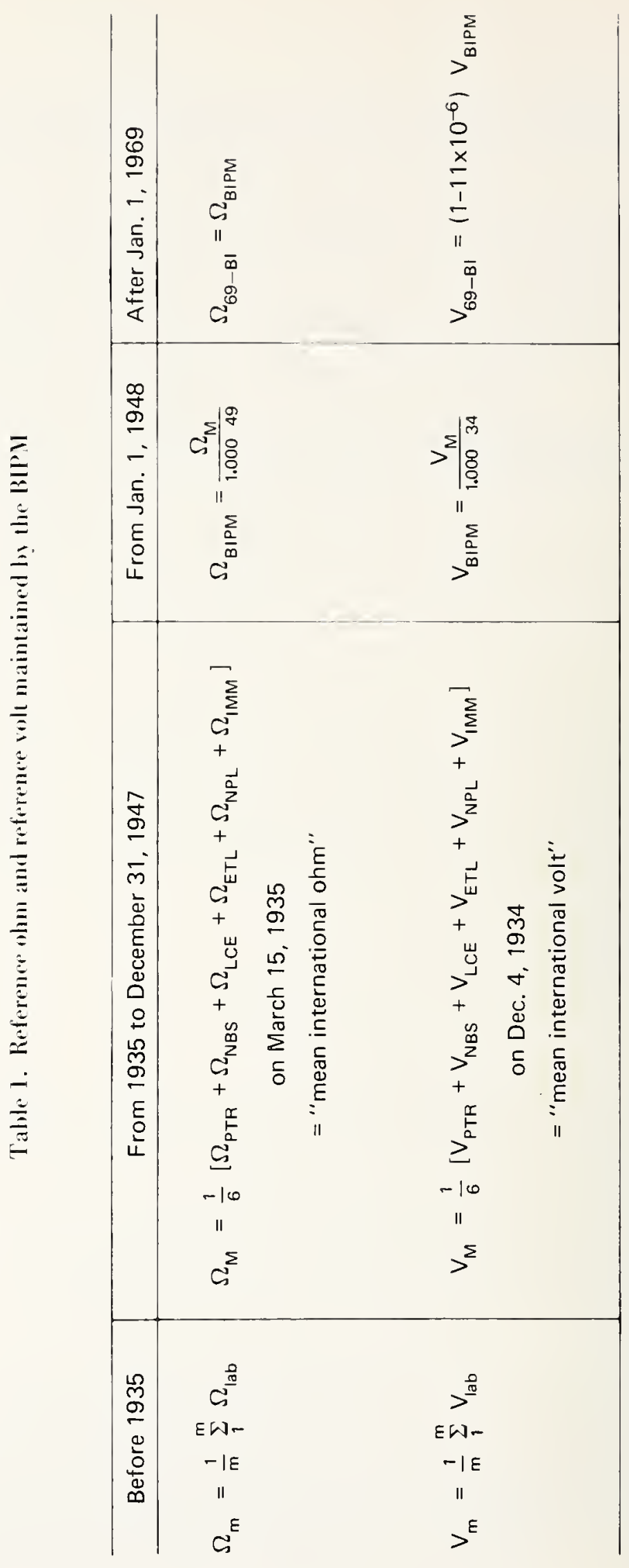


in or that one of the old ones modified the value assigned to its reference standards in the interval between two comparisons.

To avoid this serious drawback, the CCE in 1935 defined the "mean international ohm" $\left(\Omega_{\mathrm{N}}\right)$ and the "mean international volt" $\left(V_{\mathrm{M}}\right)$, as the means, on 15 March 1935 and on 4 December 1934, respectively, of the representations of the ohm and the volt maintained in the six large national laboratories of the time (PTR. NBS, LCE, RTL. NPL, and IMM). these means to be considered henceforth as constant. These are the means $\Omega_{\mathrm{M}}$ and $\mathrm{V}_{\mathrm{M}}$ which the BIPM maintained from 1935 to the 1st of January 1948, date of the transition to absolute units.

It was foreseen that the value of the standards of the BIPM would be readjusted, when necessary, on the occasion of international comparisons (and this was actually the case in 1935. 1937, and 1939); but starting in September 1939 the BIPM found itself isolated and was forced to maintain $\Omega_{\mathrm{M}}$ and $V_{n}$ depending solely on the quality of its own group of reference standards. Some later studies have shown that the behavior of these standards, kept under the best possible conditions, had been excellent; and this was fortunate for they played an important role in 1946. In fact, between 1939 and 1946, several reference standards of the six national laboratories involved were destroyed or damaged. It was thus considered in 1946 that the "international mean units" $\Omega_{M}$ and $V_{M}$ were those maintained by the standards of the BIPM. The relations then adopted by the CIPM to pass from these units to the absolute units were therefore applied directly to the references maintained by the BIPM which became, starting on 1 January 1948, $\Omega_{\mathrm{BIPM}}$ (absolute ohm maintained by the BIPM resistors) and $V_{\mathrm{BIPM}}$ (absolute volt maintained by the BIPM cells), with the transformation relations given in table 1 .

In 1946, the CIPM decided that henceforth $\Omega_{\mathrm{BIPM}}$ and $V_{\mathrm{BIPM}}$ would constitute autonomous references and would participate in the international comparisons on the same basis as the references of the national laboratories. Since 1950 the results of the international comparisons have been expressed in terms of the references maintained by the BIPM.

The CIPM also specified in 1946 that "the values to be assigned to the reference standards maintained by the BIPM would be fixed from time to time by the CIPM on the advice of the CCE, in accord with the results of the comparisons carried out between these standards and the national standards whose values had been determined directly by absolute measurements. Conforming to this decision, the CIPM decided in 1968 to correct the values assigned to the references of BIPM. To avoid confusion between the old references and the new ones, the symbols have been modified.

The relations between the references successively maintained by the BIPM are given in table 1 .

Most of the large national laboratories having simultaneously proceeded to the readjustment of the values assigned to their reference 
standards, concordance among the national representations of the ohm and the volt was assured for the first time to about one part in a million, practically throughout the entire world, the lst of January 1969.

\section{Progress of Metrology in the Field of Electricity}

After havin: been very slow and laborious for numerous years, being limited to the refinement of relatively old classical methods (use of the current balance to realize the ampere, calculation of self-inductance or mutual inductance to realize the ohm), the progress of electrical metrology has been remarkable since 1956: thanks to the application of recently discovered physical laws, the verification of the permanence of standards and the accuracy of the realizations of the units have been considerably improved.

\section{Permanence of Standards}

Up until about 1960 for the olım and 1970 for the volt, it was difficult to detect the drift of national reference groups. The relative drifts placed in evidence by the international comparisons indeed seemed to prove the instability of the standards, but the limited precision of absolute measurements (20 to 50 times less precise than the relative measurements) did not permit det ermining this instability with assurance.

\section{Resistors of Pure Metal}

To attempt the determination of the instability of manganin resistance standards which comprised its reference group, the BIPM has constructed several $1-\Omega$ standards of platinum wire, defined at the temperature of the triple point of water. From very numerous comparisons carried out regularly from 1958 to 1969 between the standards made of manganin and of platinum, the BIPM has concluded that its reference group probably remained stable to about $5 \times 10^{-7} \Omega$.

\section{Gyromagnetic Ratio of the Proton}

It has been known for several years, how to reproduce the ampere with a good precision (about $10^{-6}$ ) with the aid of the phenomenon of nuclear magnetic resonance.

For a proton (nucleus of hydrogen) of magnetic moment $\mu$ placed in a field of magnetic flux density $B$, there exist only two possible energy states: 
$\mu B$ when its magnetic moment is parallel to $B$ and $-\mu B$ when it is antiparallel. When the proton passes from one state to the other, its energy thus changes by $2 \mu B$, which involves the emission or absorption (according to the sense of the flip) of a quant um of radiation $h \nu=2 \mu B$ ( $h$, Planck's constant; $\nu$, radiation frequency). The frequency of the radiation is proportional to $B: \nu=(2 \mu / h) B$; the proportionality factor $\gamma=(2 \mu / h)$ is called the gyromagnetic ratio of the proton [4]. If the flux density $B$ is created by a current $I$ in a solenoid, it is expressed by $B=k I$ and consequently $\nu=k \gamma I$. The phenomenon of nuclear resonance then permits directly relating a current $I$ to a frequency which is well defined and easy to measure. If $k$ remains constant (that is to say, if the geometric dimensions of the solenoid and the permeability of the medium do not change), it is sufficient to reproduce $\nu$ to recover $I$.

Experimentally, the nuclear magnetic resonance of a very large number of protons is observed at the same time; besides, the larger this number is, the more convenient is the measurement. As it is necessary, however, to contain these protons in a small volume (in the region of space where the flux density is uniform), one observes in practice the nuclear resonance in a sample of pure water of several cubic centimetres $\left(6 \times 10^{22}\right.$ hydrogen atoms per $\mathrm{cm}^{3}$ ) which has been placed at the center of a long solenoid.

At the BIPM, we do not yet use the phenomenon of nuclear magnetic resonance to maintain the ampere but we use it to stabilize and measure the magnetic flux density in the air-gap of the electromagnet where we determine the energies of $\alpha$-particles (see p. 190).

\section{Application of the Josephson Effect}

To monitor the behavior of its reference cells, the BIPM has undertaken to put the Josephson effect into operation. The phenomenon, discovered in 1962 [5], manifests itself between two superconductors separated by an insulating layer of very small thickness ( 1 to $3 \mathrm{~nm}$ ) constituting a "Josephson junction"; it furnishes a relation between a direct voltage and a frequency.

In a superconductor, the conduction electrons of opposite spin group themselves by pairs (Cooper pairs). These pairs of electrons do not obey the same statistics as do independent electrons; they have a tendency to put themselves into the same quantum state so that a single wave function $\phi=\phi_{0} \mathrm{e}^{i \theta}$ (called the order parameter) suffices for characterization. As a result, one can observe a quantum effect on the macroscopic scale.

When the insulating barrier which separates two superconductors is sufficiently weak that the phases $\theta_{2}$ and $\theta_{1}$ of the order parameters appropriate to each of them cannot vary independently, some electron pairs 
can cross the barrier by the tunnel effect. The current $I$ which results, and the time variation of the difference between the phases have for their expression:

$$
I=I_{\mathrm{c}} \sin \left(\theta_{2}-\theta_{1}\right)
$$

and

$$
\frac{\mathrm{d}\left(\theta_{2}-\theta_{1}\right)}{\mathrm{d} t}=\frac{2 e}{h} U
$$

where

$I_{\mathrm{c}}=$ critical current whose value depends mainly on the nature of the junction and on the magnetic field in which it is placed,

$e=$ elementary charge

$h=$ Planck's constant

$U=$ potential difference across the terminals of the junction.

If one passes a bias current $I$ through such a junction, the phases $\theta_{1}$ and $\theta_{2}$ adjust themselves to satisfy equation (1) and no voltage appears at the terminals of the junction as long as $I \leqslant I_{\mathrm{c}}$ (this is the d-c Josephson effect). When $I$ becomes greater than $I_{\mathrm{c}}$ a voltage $U$ appears and the difference between the phases $\left(\theta_{2}-\theta_{1}\right)$ increases proportionally with the time; by this fact the junction becomes the seat of an alternating current of frequency $\nu=(2 e / h) U$ (this is the a-c Josephson effect which relates $U$ to $\nu$ through the natural constants $e$ and $h$ ).

If the bias current $I$ is progressively increased while the junction is irradiated with an electromagnetic radiation of frequency $\nu$, the voltage $U$ across the terminals of the junction increases discontinuously and exhibits steps whose levels are multiples of $h / 2 e \nu$ (figs. 12a and 12b). With the value
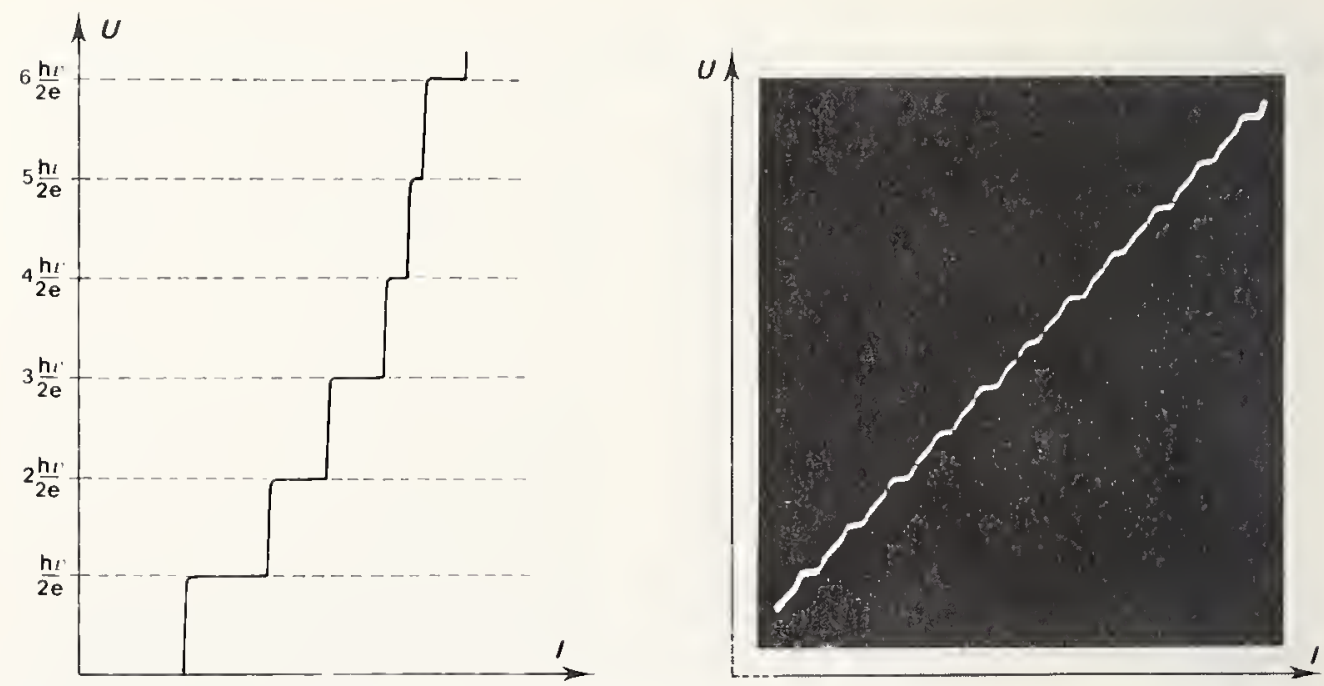

Figure 12. A-c Josephson effect. a: theoretical characteristic $U=f(1)=n(h \nu / 2 e)$; b: characteristic observed in the neighborhood of the $250 \mathrm{th}$ step (about $5 \mathrm{mV}$ ); step width in current, about $20 \mu \mathrm{A}$. 
$2 e / h=483594 \mathrm{GHz} / \mathrm{V}$ and for a frequency of incident radiation $\nu=9.67$ $\mathrm{GHz}$, the steps are equidistant in the neighborhood of $20 \mu \mathrm{V}$.

The relation $U=n(h / 2 e) \nu$ is independent of the nature of the superconductors and the barrier, of the temperature, of the magnetic field, and of the power of the incident radiation.

The Josephson voltages are however always small (no more than a few millivolts), thus difficult to measure with high precision. They are nccessarily produced at a very low temperature $(4.2 \mathrm{~K}$ or less), while the cells to which they are to be compared are maintained at $20{ }^{\circ} \mathrm{C}(293 \mathrm{~K})$ or $30{ }^{\circ} \mathrm{C}$ ( 303 $\mathrm{K}$ ) ; thermoclectric voltages necessarily result, whose influence must be eliminated as completely as possible under pain of introducing systematic errors into the measurements. Finally, the precision of the comparison depends directly on that of the voltage comparator.

At the $\mathrm{BIPM}$, we fabricate junctions $\mathrm{Pb}-\mathrm{Pb}_{x} \mathrm{O}_{y}-\mathrm{Pb}$ or $\mathrm{Pb}-$ $\mathrm{Al}_{x} \mathrm{O}_{y}-\mathrm{Pb}$ (fig. 13) (barrier resistance 200 to $300 \mathrm{~m} \Omega$ : step height. 50 to $100 \mu \mathrm{A})$, with which we can produce conveniently usable Josephson volt age of $5 \mathrm{mV}$ (for example, the 250th step). We put two junctions in series to finally make available a voltage of $10 \mathrm{mV}$.

The voltage comparator realizes to about $1 \times 10^{-8}$ the $100 / 1$ ratio which exists between the electromotive force of the cell under study and the Josephson voltage. It is constituted of 2 "Hamon devices" [6] placed in series and each comprising $10100-\Omega$ resistors; the 10 resistors of the first device are connected in parallel (resulting resistance $10 \Omega$ ) when the $10 \mathrm{re}$ sistors of the second are connected in series (resulting resistance $1000 \Omega$ ) and conversely.
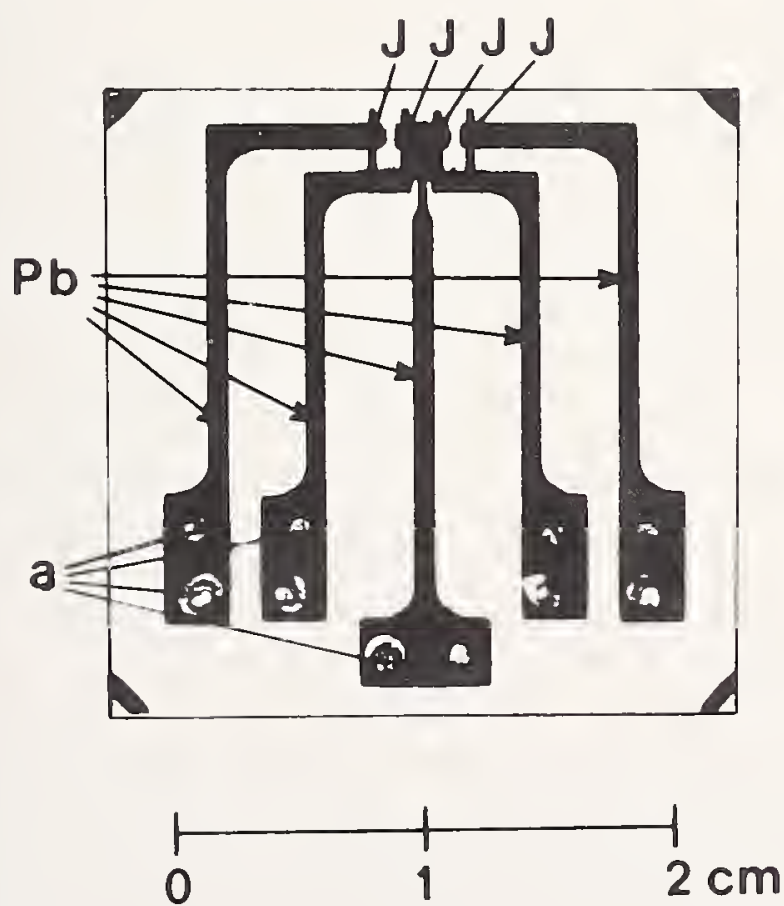

Figure 13. Small glass plate carrying four Josephson junctions. J. junction: l'b, learl: a, suldering points for current and volt age leads. 
We eliminate the effect of thermal electromotive forces by repeatirg the measurements after having reversed the bias current through the junctions, the current in the comparator, and the polarity of the cell under study.

A diagram of the principle of the installation at the BIPM is given in figure 14. This installation is placed inside a screened room to shield it from stray electromagnetic radiation.

The cells which are compared to the Josephson voltage are maintained in the temperature-regulated enclosure at $30^{\circ} \mathrm{C}$ located in the screened room and they are periodically calibrated relative to the BIPM reference cells.

During the measurements, the temperature of the helium bath in which junctions are immersed is lowered by pumping to about $1.5 \mathrm{~K}$. Below the lambda point $(2.17 \mathrm{~K})$ the helium in fact becomes superfluid and its thermal conductivity exceptionally high (200 times better than that of copper); the uniformity of the bath temperature is considerably improved and the loss of helium reduced.

The microwave-frequency electromagnetic radiation is furnished by a klystron where frequency is held constant to a few parts in $10^{9}$ throughout

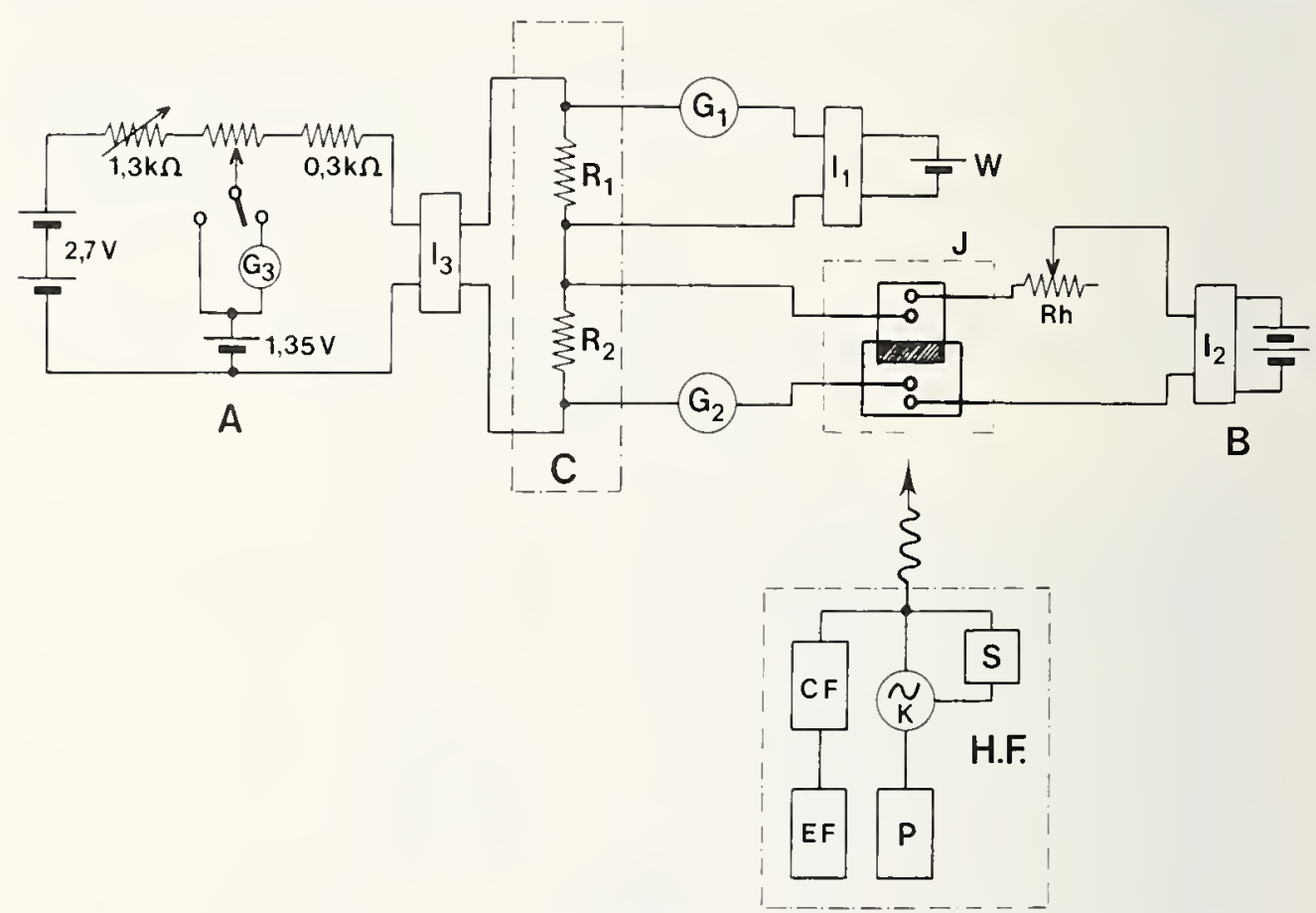

Figure 14. Diagram of the principle of the installation for the comparison of the Josephson voltage with the electromotive force of a standard cell. A. source of constant current for the voltage comparator; $B$. source of bias current for the junction; C. voltage comparator: J. junction: $W$. standard cell (electromotive force $E$ ); $I_{1}, I_{2}, I_{3}$ current reversers: $G_{1}, G_{2} . G_{3}$. balance detectors; H.F., source of microwave radiation (K, klystron; P, controlled supply for the klystron: S. frequency stabilizer; CF, frequency counter; EF. frequency standard). At balance (null current in $G_{1}$ and $G_{2}$ ) the potential difference across the junction terminals is equal to $E R_{2} / R_{1}$. 
the whole measurement by a stabilizer which acts on the polarization volt age. The frequencies used are of the order of $8.5 \mathrm{GHz}$ for the $\mathrm{Pb}-\mathrm{Pb}_{x} \mathrm{O}_{y}-\mathrm{Pb}$ junctions and of $9.5 \mathrm{GHz}$ for the $\mathrm{Pb}-\mathrm{Al}_{x} \mathrm{O}_{y}-\mathrm{Pb}$ junctions, and the irradiation power is in the neighborhood of $200 \mathrm{~mW}$.

The frequency counter is calibrated by means of a quartz clock, itself calibrated starting from the $200 \mathrm{kHz}$ transmissions of the station at Droitwich (United Kingdom). The measured frequencies are known to about $1 \times$ $10^{-9}$.

The BIPM continues to maintain the volt by means of standard cells, but the electromotive force of these cells is periodically redetermined by comparison with the reference voltage of a Josephson junction. We hope to thus be able to assure the permanence of the unit of electromotive force maint ained by the BIPM to about $1 \times 10^{-7} \mathrm{~V}$.

\section{Accuracy of Realization of the Units}

Although the BIPM does not carry out absolute measurements, it very closely follows the results and advancements of measurements made in the national laboratories; these results in fact enter into the international com parison and serve to fix the values of the BIPM reference standards; as to advancements, they guide the future activities of the BIPM.

\section{Unit of Resistance}

A very important advance has been realized in the determination of the farad and, as a consequence, in that of the ohm. Three reasons have combined for this advance.

The first and essential reason was the discovery in 1955 by A. M. Thompson and D. G. Lampard, of the NSL (Austratia), of a new electrostatic theorem [7] permitting rigorously the reduction of the determination of the capacitance of a capacitor of appropriate type to the determination of a length, assuming the permittivity of space, $\epsilon_{0}$, known. In the most highly perfected apparatus [8], the length to be measured is that of a displacement; it is determined by interference measurements in terms of the standard radiation of krypton 86 with a precision around $1 \times 10^{-8}$.

There was next the development of transformer comparator bridges [9] thanks to which one can pass from very small capacitances $(0.25$ to 0.50 $\mathrm{pF}$ ) determined in absolute value to capacitances of practical value (up to $0.1 \mu \mathrm{F}$ ) without loss of precision.

There was finally, more recently, the new determinations of the speed of light to about $4 \times 10^{-9}(c=299792458 \mathrm{~m} / \mathrm{s})$ [10], from which is deduced the permittivity of space by the relation $\epsilon_{0}=10^{7} / 4 \pi c^{2}$. 
The conjunction of these three advances today makes the farad, formerly without metrological interest, the electric unit best known in absolute value. It is thus the farad, and no longer the henry, from which one now derives the value of the ohm through the intermediary of a frequency. The precision thus obtained is in the neighborhood of $1 \times 10^{-}$; it has been improved by a factor of 30 in about 15 years.

The interest in capacitors of small value is such that they will probably, in the near future, be objects for international comparisons of the same kind as standards of resistance and of electromotive force. The BIPM has consequently equipped itself for carrying out comparisons of capacitors of 1,10 , and $100 \mathrm{pF}$ with a precision of about $1 \times 10^{-8}$.

\section{Unit of Electromotive Force}

The absolute determination of the volt has similarly profited from the advances made in the determination of the farad. The possibility of replacing the always difficult and uncertain calculation of a capacitor by its measurement has given a fresh impetus to the so-called "absolute electrometer" in which the potential $U$ applied to the movable member of a capacitor is deduced from the measurement of a force $F$ and the variation $\delta C$ of the capacitance in terms of the relative displacenent $\delta x$ of the movable member $\left(F=\frac{1}{2} \frac{\delta C}{\delta x} U^{2}\right)$. The precision of the determinations underway in several national laboratories could reach $1 \times 10^{-6}$, that is to say, three to four times better than the precision currently obtained.

\section{Conclusion}

Following the mission which was assigned by the CIPM in 1929, the BIPM has for almost a half century assured the uniformity of electrical measurements throughout the world. Applying without delay the advances of science and technology, it has continually improved its installations or created new ones in order that its standards permanently represent the ohm and the volt as exactly as possible. In 1939, the CCE estimated that the uncertainty was $2 \times 10^{-5}$ for the ohm and 1 or $2 \times 10^{-4}$ for the volt; in 1948 these uncertainties were reduced to $1 \times 10^{-5}$ for the ohm and $2 \times 10^{-5}$ and a few in $10^{6}$. Progress has thus been continual.

These successes have led several national laboratories to request the CIPM to extend the competence of the BIPM to measurements of electric quantities in the domain of radiofrequencies. The CIPM received this request favorably and in 1964 approved the creation within the $\mathrm{CCE}$ of a permanent working group for radiofrequency quantities. Without participating in the work experimentally, the BIPM has coordinated, since 1965, the execution of several international comparisons carried out in this domain by the specialized laboratories. 
The results already obtained are satisfactory and we believe that in this new discipline in full development. international collaboration will permit rapid improvement in the consistency and precision of measurement results.

\section{Bibliography}

[1] Procès-Verbaux C.I.P.M. 13, p. 58 (1929).

[2] Procès-Verbaux C.I.P.M. 20,p. $129(1945-1946)$.

[3] Comptes Rendus 9" C.G.P.M., p. 49 (1948).

[4] Purcell, E. M., Torrey, H. C., and Pound, R. V., Resonance absorption by nuclear magnetic moments in a solid, Phys. Rev. 69, pp. 37-38 (1946).

Bloch, F., Hansen, W. W., and Packard, M., Nuclear induction, Phys. Rev. 70, pp. 460-474 (1946).

[5] Josephsom, B. D., Phys. Letters 1, p. 251 (1962).

Langenberg, D. N., Scalapino, D. J., and Taylor, B. N., The Jusephson effects, Scientific American, pp. 30-39 (May 1966).

Taylor. B. N., Parker. W. H., Langenberg, D. N., and Denenstein, A., On the use of the $\mathrm{AC}$ Josephson effect to maintain standards of electromotice force, Metrologia $\mathbf{3}$, No. 4. pp. $89-98$ (1967).

Petley, B. W. and Morris, K., A measurement of $2 e / h$ by the AC Josephson effect, Metrologia 6, No. 2, pp. 46-51 (1970).

[6] Hamon. B. V., A 1-100 $\Omega$ build-up resistor for the calibration of standard resistors, J. Sci. Instr. 31 , No. 12, pp. 450-453 (1954).

Page, Ch. H., Errors in the series-parallel buildup of four-terminal resistors, J. Res. Nat. Bur. Stand. (U.S.) 69C, No. 3, pp. 181-189 (1965).

Page, Ch. H.. Errors in the series-parallel buildup of four-terminal resistors, J. Res. Nat. Bur. Stand. (U.S.) 69C, No. 3, pp. $181-189$ (1965).

Page, Ch. H., Tetrahedral junction error contribution to a series-parallel four-terminal resistor, IEEE Trans. Instrum. Meas. IM-23, No. 1, pp. 5-8 (1974).

Harris, F. K., Fowler, H. A., and Olsen, l’. T., Accurate Hamon-pair potentiometer for Josephson frequency-to-voltage measurements. Metrologia 6, No. 4, pp. 134.142 (1970). Finnegan, T. F. and Denenstein, A., High accuracy potentiometers for use with ten millivolt Josephson devices. I. Double series-parallel exchange comparator, Rev. Sci. Instr. 44, No. 8, pp. 944-953 (1973).

[7] Thompson, A. M. and Lampard, D. G., A new theorem in electrostatics and its application to calculable standards of capacitance, Nature 177 , p. 888 (1956).

Lampard, D. G., A new theorem in electrostatics with applications to calculable standards of capacitance, Proc. Inst. El. Engrs., Monograph 216M, 104C, 10 pages (1957).

[8] Clothier, W. K., A calculable standard of capacitance, Metrologia 1, No. 2, pp. 36-55 (1965).

[9] Thompson, A. M., The precise measurement of small capacitances, IRE Trans. on Instrumentation 1-7, Nos. 3-4, pp. 245-253(1958).

McGregor, M. C., Hersh, J. F., Cutkosky, R. D., Harris, F. K., and Kotter, F. R., New apparatus at the National Bureau of Standards for absolute capacitance measurement, IRE Trans. on Instrumentation 1-7, Nos. 3-4, pp. 253-261 (1958).

.[10] Terrien. J., International agreement on the value of the velocity of light, Metrologia 10 , p. 9 (1974). 



\section{CHAPTER VIII}

\section{PHOTOMETRY}

When, in 1875, the BIPM was created to look after length and mass, T. Edison had not yet constructed the first incandescent lamp. It was, however, already a century and a half since P. Bouguer had published his Essai d'optique sur la gradation de la lumière, which can without doubt be considered as the first serious attempt at visual photometry; the luminous sources of that time were flames and even the name of the various units in use up to 1948 strangely recalls the first standards: candles. There have been successively lamps using coleseed oil (Carcel), pentane gas (VernonHarcourt), amyl acetate (Hefner); in all these cases the luminous intensity depended not only on the fuel, but also on the wick, the height of flame, etc. To avoid the inconveniences of flame standards, Violle proposed in 1879 to adopt the luminous intensity emitted from $1 \mathrm{~cm}^{2}$ of the surface of a bath of pure platinum in the course of solidification; unhappily, and for various reasons (impurities in the platinum, for example), Violle's standard had low reproducibility.

Artificial illumination became practical and effective only with the appearance of the incandescent lamp with a carbon filament (1879) and the invention of the gas mantle (1895); it was the rivalry between these two sources that made photometry progress.

Originally, each country had its own, and rather poorly reproducible, unit of luminous intensity; it was necessary to wait until 1909 to see a beginning of unification on the international level, when the national laboratories of the United States of America, France, and Great Britain decided to adopt the international candle represented by carbon filament lamps; Germany, at the same time, stayed with the Hefner candle, defined by a flame standard, and equal to about nine-tenths of an international candle. But a standard based on incandescent lamps, and consequently dependent upon their stability, would never have been fully satisfactory and could therefore be only provisional; on the other hand, the properties of a blackbody provided a theoretically perfect solution and, as early as 1933 . the principle was adopted that new photometric units would be based on the luminous emission of a blackbody at the freezing temperature of platinum $(2045 \mathrm{~K})$. 
It was in this situation, perhaps a little confused, but full of hope, that the CIPM decided in 1937 to extend the activities of the BIPM and to install a photometric laboratory in the Pavillon de Breteuil, in order to "contribute to the international unification of the important physical quantity which is the unit of light.'

The role of the BIPM was not, and is no more so today, to realize the primary standard (blackbody), but to maintain secondary reference standards for practical use in the form of standard incandescent lamps having carbon or tungsten filaments; the values assigned to these lamps were to be expressed with the aid of the "mean unit" that results from determinations made in all national laboratories that have realized the primary standard.

Naturally, photometry is much more than the comparison of luminous sources. Associated with radiometry, its applications extend to many other disciplines such as colorimetry, optics, photography, astronomy, pyrometry, etc. But here as elsewhere, the role of BIPM is definite and volunt arily limited to the basic metrology.

\section{Apparatus and Measurement Methods at the BIPM}

The laboratory of the BIPM created in 1937 was thus conceived to respond to these needs; its equipment can be divided into two entirely distinct parts: the electrical facility for providing power to the lamps and the specifically photometric installations.

The direct current electrical supply of the lamps is simple since a relative precision of a few parts in $10^{5}$ is sufficient; initially assured by lead storage batteries of large capacity, the supply is now provided from the power lines through the intermediary of rectifiers and stabilizers, giving a more versatile operation.

A single potentiometer permits simultaneous determination of the potential difference between the lamp terminals (by the intermediary of a voltage divider, however) and the current through the lamp; for the latter. one measures the potential difference between the terminals of a calibrated resistor connected in series with the lamp (fig. 1). Two lamps can be supplied simultaneously on completely independent electrical circuits, this was originally required by the measurement techniques used, and remains today a practical advantage.

The photometric measurement of lamps at the BIPM displays a characteristic which results from the coordinator role played by the BIFM and which greatly facilit ates the comparisons; all the lamps to be compared are always operated at the same distribution temperature, ${ }^{* 1}$ that is to say that their radiations have just about the same color; it is therefore not necessary, at least in principle, to verify the spectral properties of the detector, whether it is the human eye or a physical sensor.

\footnotetext{
1 The terms marked with an asterisk are defined in the lexicon.
} 


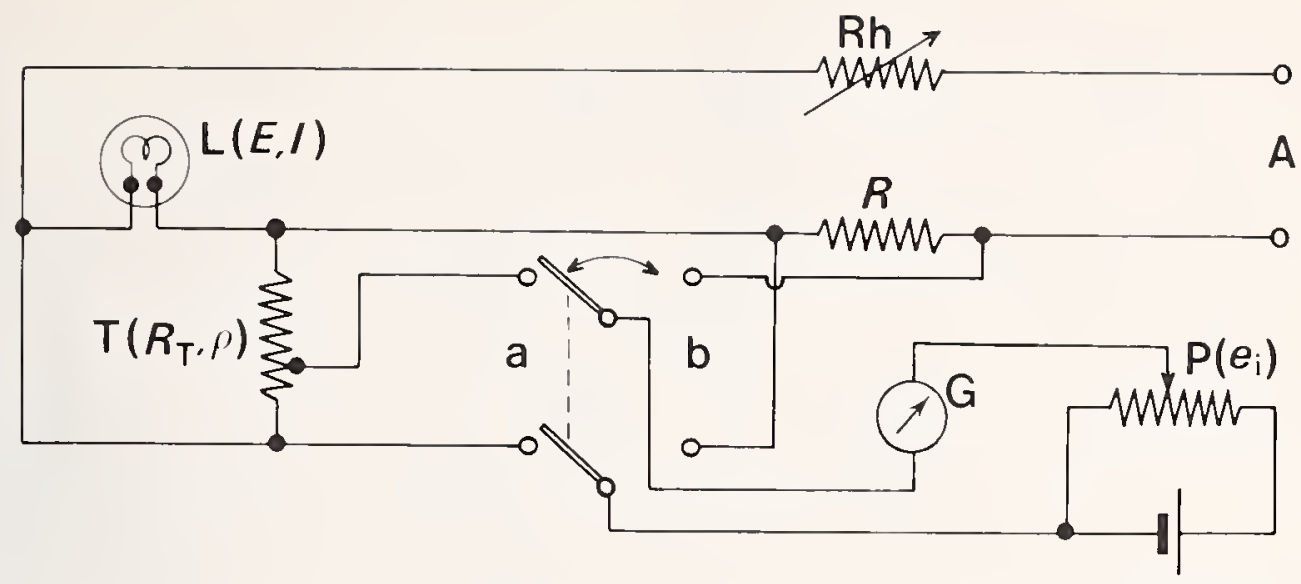

Figure 1. Neasurement of the electrical characteristice of an incandescent lamp. L. lamp: $R$. calibrated resistor: $A$, adjustable stabilized supply (direct current): Rh. fine arljustment rhenstat: T, voltage divider of total resistance $R_{\mathrm{T}}$ and of division ratio $\rho=0.01$ : P. meanuring potentiometer: (i, tralvanometer.

a) The reading e corresponds to the potential clifference E between the terminals of $L$ :

$$
E=\rho_{1} / \rho . \text { wr } E=100 e_{1}
$$

$(E$ is generally between 10 and $200 \mathrm{~V}$.)

b) The reading e. corresponds to the current $/$ through $L$ :

$$
I=e_{2} / R-E / R_{\mathrm{T}}
$$

( $/$ is generally between 0.2 and $6 \mathrm{~A}$.)

Photometric measurement [1] depends on either standards of huminous intensity* or standards of luminous flux*: thus a difference of only a geometric nature separates the two kinds of measurement. Standards of intensity are placed directly on a photometric bench in front of the sensor; standards of flux are placed in the center of a sphere (fig. 2) provided with a small observation window and coated on the interior with a diffusin white coating; the window faces the sensor. In the two cases, the measurement itself is finally the same and consist in attributing a value to the ratio of illuminances* received by the sensor in comparing the two standard lamps.

Originally the measurements were visual; the eye can judge correctly only the equality of two illuminances, which must therefore be realized simultaneously by using an auxiliary comparison lamp: the system for adjustment of the illuminance utilized variation of the distance to the photometer, either from the comparison lamp or from the standard lamp (fig. 3). Since the spectral sensitivity of the eye is not rigorously identical from one individual to another, it was necessary either to have the measurements made by a large number of persons or to use several observers who possessed the "average eye," at the least for a time and under certain conditions. This "average eye" has been well defined physically since 


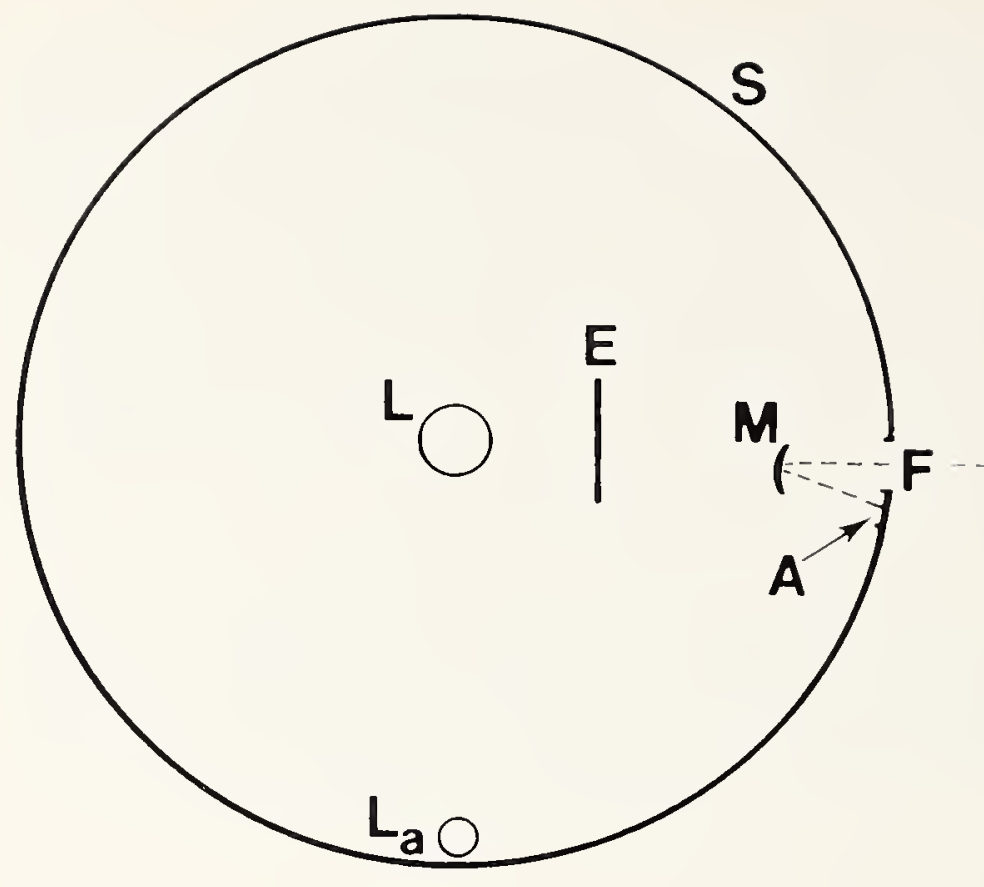

R

Figure 2. Integrating sphere. This device is used for comparing st andards of luminous flux. S, sphere (1.5 m diameter): L, standard lamp; $L_{i a}$, auxiliary lamp: E. opaque screen $(15 \mathrm{~cm}$ diametcr); I, spherical mirror; F, observation window (4 cm diameter): R, photoelectric sensor: A, area of the interior wall of the sphere, seen by R, via $M$. E protects $F$ and A from direct radiation emitted by $\mathrm{L}$. The illuminance at $\mathrm{A}$ i s proportional to the luminous flux from L.

The interior wall surface of the sphere carries a coating which should be uniform. stable. perfectly diffusing: and with a nonselective high reflection factor. It is difficult to unite these diverse propertios, but a flat white paint having zinc oxide pioment constitutes a satisfactory compromise.

The lamp $L$ placed at the center of the sphere must be considered as a foreign body which "alosorbs" a part of its own luminous flux; since this ahsorption is different from one lamp to another, one is led to apply a correction (rarely more than $1 \%$ ) whose determination is provided by $L_{a}$ (measurements of the flux of $L_{a}$ with and without $L$ in the sphere). 
(a)

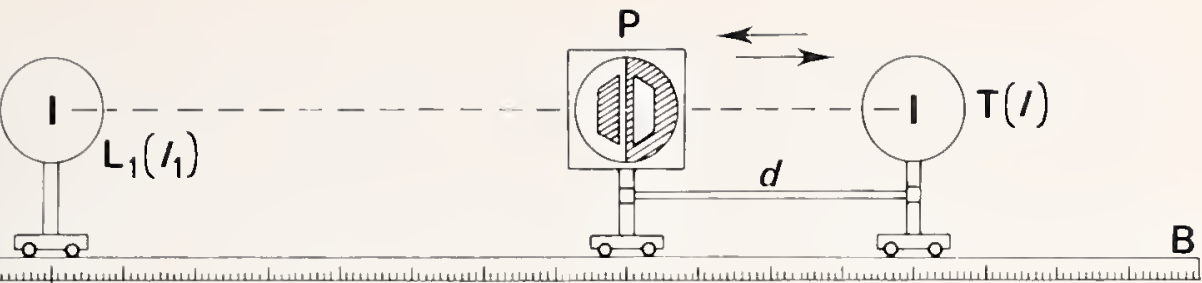

0

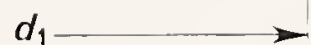

(b)

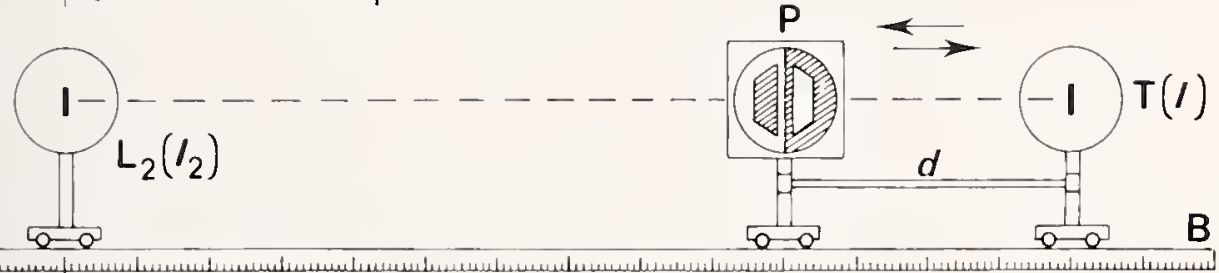

0

$d_{2}$

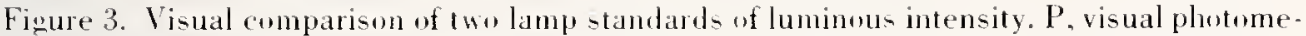
ter: T. comparison lamp (of luminous intensity $I$ ): B, photometric bench furnished with a graduated scale: $L_{1}$, reference lamp (of known luminous intensity $I_{1}$ ); $L_{2}$, lamp to be calibrated (of unknown luminous intensity $I_{2}$ ). P and T. movable along B. are rigidly linked to each other at the fixed listance $d$, which it is not necessary to know. In the course of each of the two operations (a) and (b), one displaces the ensemble (P,T) just to the point where the eye estimates that there are equal illuminances at $P$; one then has:

$$
I_{1} / d_{1}^{2}=I / d^{2} \text { and } I_{2} / d 2^{2}=I / d^{2}
$$

whence:

$$
I_{2}=I_{1}\left(\frac{d_{2}}{d_{1}}\right)^{2}
$$


1924, thanks to an international agreement bearing on the curve of relative spectral luminous efficacy $V(\lambda)$ (fig. 4); photometry is then completely freed from physiological influence and has thus become a major scientific discipline. similar to thermontetry for example. Even aside from the fact that visual measurements were long and painstaking, their duration presented a definite inconvenience because incandescent lamps are standards of a shightly peculiar character: they change, as a matter of fact. although very slowly, while being used.

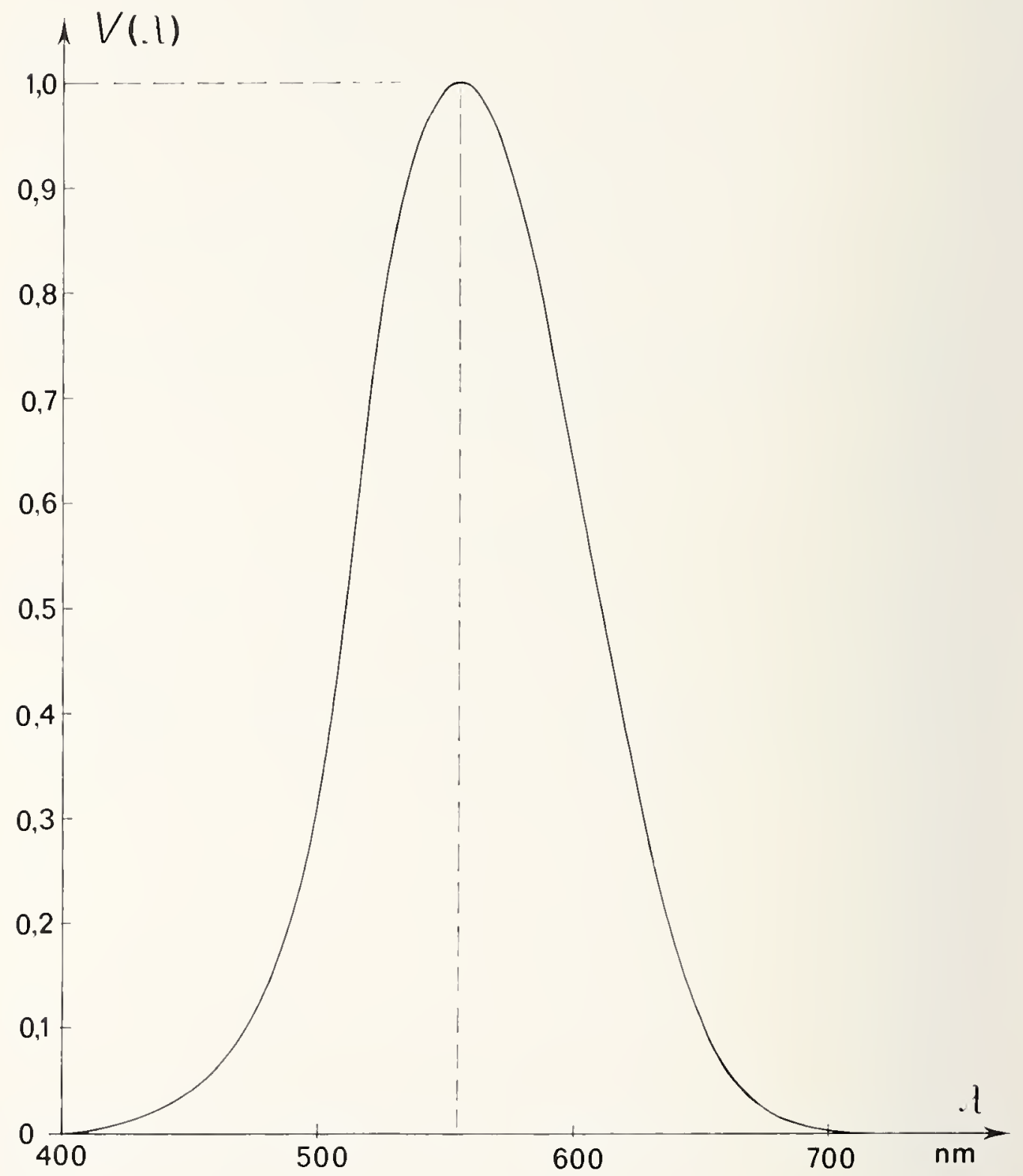

Figure 4. Relative spectral luminous efficacy $V(\lambda)$. The values adopted by the Commission Internationale de l'Eclairage (C.I.E.) are given in the form of a table [2], at $1 \mathrm{~nm}$ wavelength intervals from 360 to $830 \mathrm{~nm} . V(\lambda)$ is valid for photopic vision (or daytime vision) only: there is another curve $V^{\prime}(\lambda)$ applicable to scotopic vision (night-time vision). 
A substitute solution was therefore sought; nonsubjective and more rapid, and there soon followed the adoption of the selenium photovoltaic cell which, under certain conditions of use. constitutes a satisfactory sensor for photometry. The photovoltaic cell is used in the same way as the eye. for determining the equality of two illuminances, the determination of the ratio of two standards always resulting from the measurement of distances to the sensor.

A new advance was achieved with the photoelectric cell, today still in service. The vacuum cell, of the Gillod-Boutry type (cylindrical anode forming a Faraday cage around the cathode), presents the enormous advantage of excellent linearity, better than $10^{-4}$ for an illuminance ratio between 1 and 2 for example; this remarkable quality, together with the fact that the illuminances to be compared no longer need be realized simultaneously, but on the contrary, should be realized successively, has permitted simplifying the measurement installation (fig. 5). A comparison lamp is no longer needed, and the measurement of distances is no longer required; the current given by the cell is proportional to the illuminance on the sensor. A potentiometric compensating device, associated with a direct-current amplifier using a null detector, completes the receiver. Although the incandescent lamps to be compared have identical spectral energy distributions in principle, it has been judged prudent to correct the spectral sensitivity of the cell by means of filters to make it approximately analogous to $V(\lambda)$ thus avoiding a small uncertainty due to the minor differences in spectral distribution of the radiation from the lamps.

This measurement method is rapid, which is beneficial for the standard lamps; moreover, it permits total freedom from the law of variation of illuminance as a function of distance, a law whose rigorous validity cannot be guaranteed (source and receiver of non-negligible dimensions compared

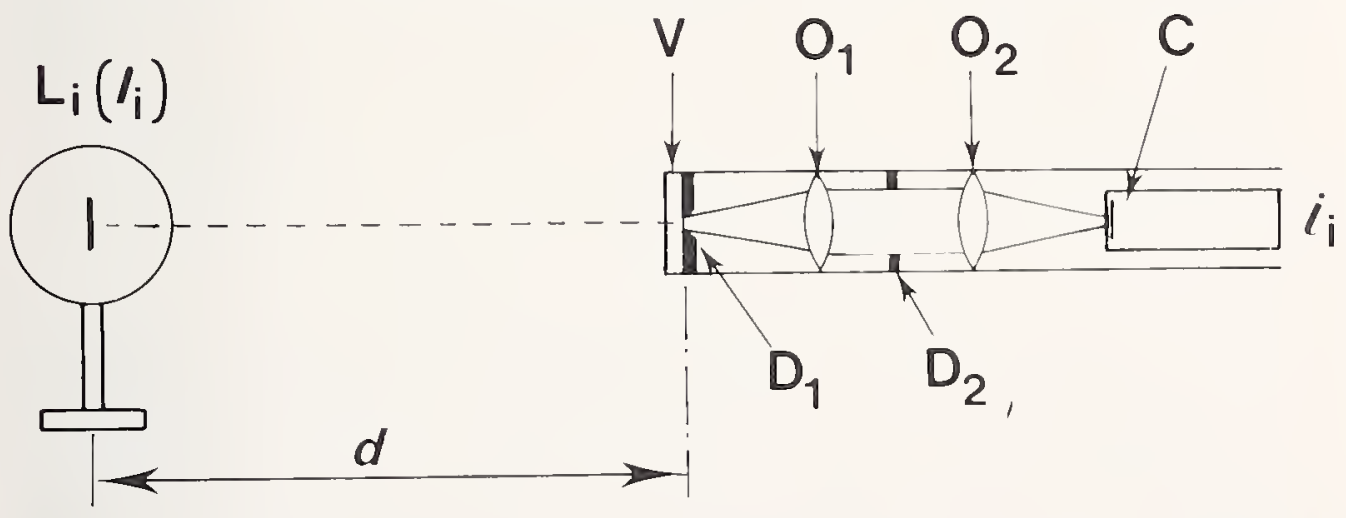

Figure 5. Photoelectric comparison of two lamp standards of luminous intensity. $V$, double opal glass: $D_{1}, D_{2}$, diaphragms; $\mathrm{O}_{1}$. $\mathrm{O}_{2}$ objectives: $\mathrm{C}$. cathode of the photoelectric tube. The reference lamp $L_{1}$ lof known luminous intensity $I_{1}$ ), then the lamp to be calibrated $L_{2}$ (of unknown luminous intensity $\left.I_{2}\right)$ are successively placed at the unknown. hut fixed. distance $d$ in front of $\mathrm{V} . \mathrm{D}_{1}$ defines the surface of the cathode which is illuminated and. with $\mathrm{D}_{2,}$ the geometrical throughput of the beam. The photoelectric current $i_{1}$ is proportional to $I_{\mathrm{i}}$. which implies:

$$
I_{2}=I_{1}\left(i_{2} / i_{1}\right)
$$


to the distance that separates them, atmospheric absorption). It is cstimated that the precision of measurement is better than 0.1 percent, when all the precautions in the organization of the measurements, symmetry in particular, are taken.

\section{International Comparisons of Intensity and of Luminous Flux}

The BIPM has already utilized its installations five times $(1948,1952$, 1956, 1961, 1969) for comparisons of standard lamps which maintain, in the large national laboratories, the two principal photomctric units [3]; these two units are the candela*, which is either determined directly starting with the primary standard at $2045 \mathrm{~K}$ (fix. 6), or realized at higher distribution temperatures $(2357 \mathrm{~K}$ and $2859 \mathrm{~K})$, and the lumen* realized at $2357 \mathrm{~K}$ or at $2793 \mathrm{~K}$ (fig. 7). The laboratories participating in these comparisons are

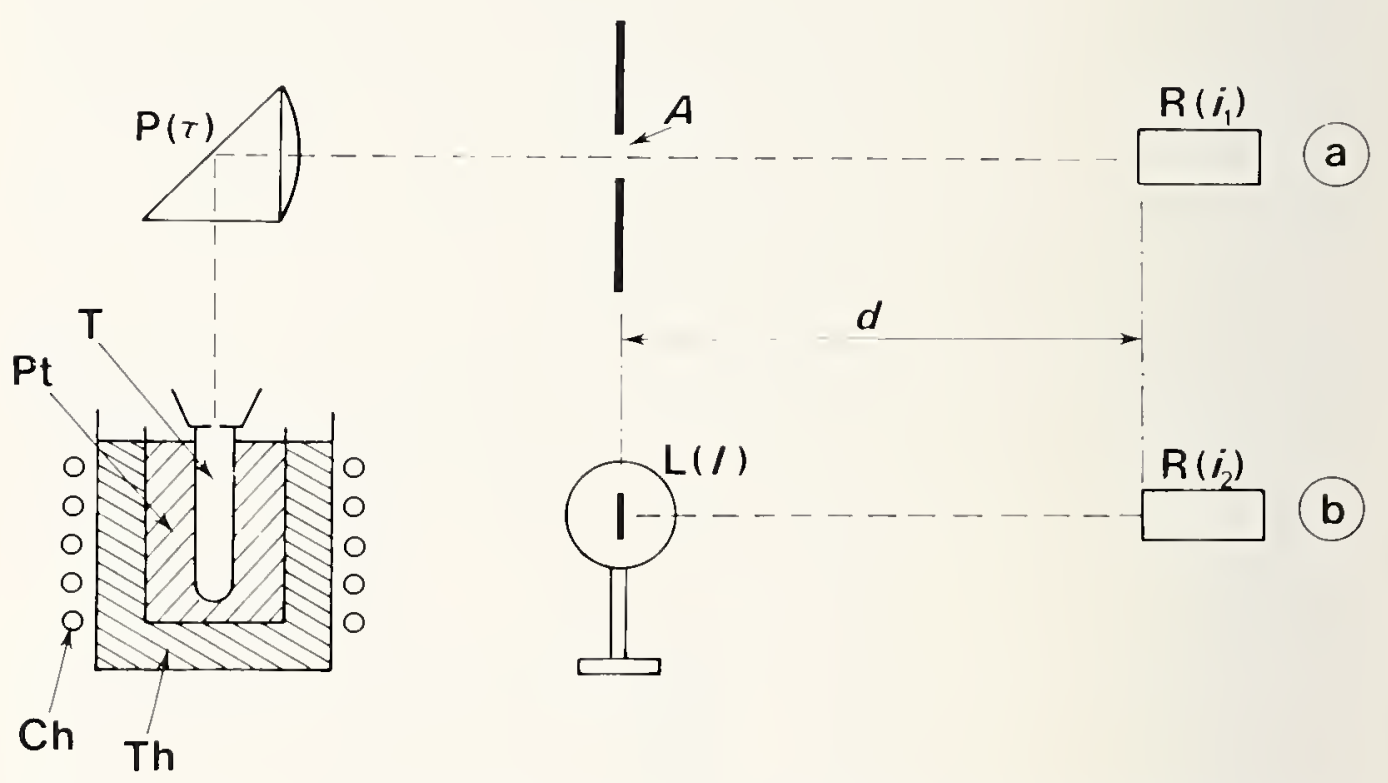

Finure 6. Principle of the calibration of an incandescent lamp ly comparison with the primary standard of luminous intensity $(2045 \mathrm{~K})$. T. Backhody (thoria tube): Pt. pure platinum at its freezing temperature: Th, thoria powder: Ch. heater: 1'. prism-lens of transmission factor $\tau: A$, area (to be determined precindy) of the aperture of a diaphragm; R. photoelectric sensor whose current $i_{1}$ is proportimal on the received illuminance $E_{\mathrm{i}}$ : $L$, incandescent lamp of luminous intensity $l$ to be determined: $d$. distance which need not be known, but which must lec constant.

(a) $E_{1}=600000\left(\tau \cdot A / d^{2}\right)$. from the definition of the candela

(b) $E_{2}=\left(1 / d^{2}\right)$

so that

or

$$
I=600000 \tau \cdot A\left(E_{2} / E_{1}\right)
$$

$$
I=600000 \tau \cdot A\left(i_{2} / i_{1}\right)
$$


in principle those which have realized the primary standard, but the magnitude of the work to be done for this realization is such that there are rarely more than one or two laboratories that have redetermined the candela starting with the blackbody before each new comparison.

The results obtained show some disagreements between laboratories of as much as about \pm 0.8 percent for the candela determined directly from the primary standard; this is much larger than the errors (about 0.3\%) which each laboratory estimates, which proves that there exist important systematic errors in the blackbody realization or in the transfer to incandescent standards; studies undertaken on the causes of these errors have not yet permitted reducing the differences.

Under these conditions, it might be feared that the spread among the results of the laboratories may be larger for standards of huminous intensity at higher distribution temperatures, and for standards of flux; this is happily not the case, without doubt by a phenomenon of partial error compensation, since the disagreements do not exceed \pm 1.1 percent (fig. 8 ). But perhaps the most important verification is that in the chain of comparisons
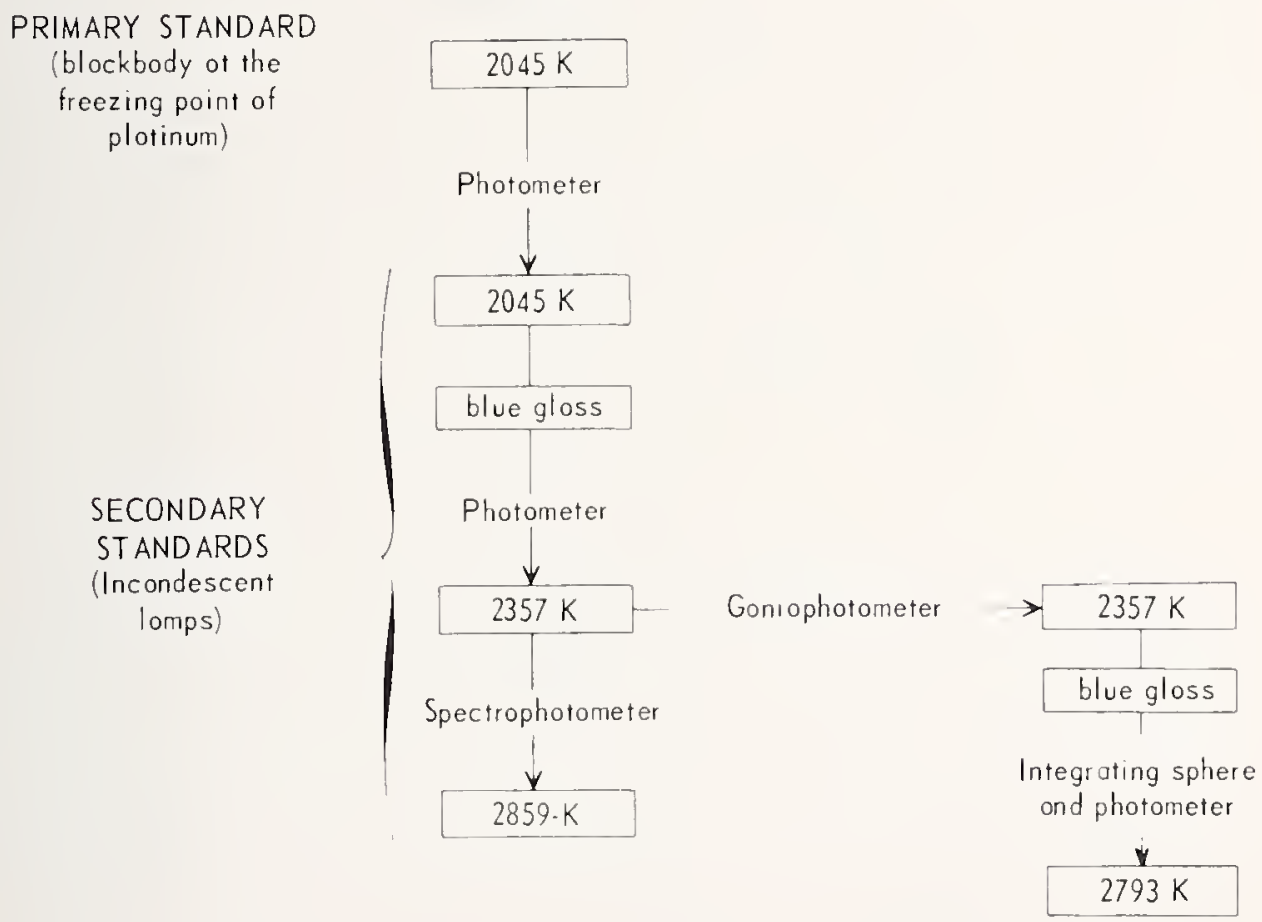

Figure 7. Genealogy of photometric standarls. The origin of the various values of distribution temperature attributed to the standards is the following: $2045 \mathrm{~K}$ - freezing tomperature of platinum: $2357 \mathrm{~K}$ - temperature olstained by placing a certain blue glass in front of a standard at $2045 \mathrm{~K}: 2793 \mathrm{~K}$ - temperature obtained by placinctle same blue wlass in front of a standard at $2357 \mathrm{~K} ; 2859 \mathrm{~K}$ - temperature of a colorimetric reference standard. (The arrows indicate the passage from one standart to the n'st, with the comparison instrument indicated.) 
there has appeared no reduction in the dispersion of the results between national laboratories: we have, however, in particular for the comparison of 1969, tried to get rid of some defects and uncertainties of incandescent lamps by multiplying the precautions taken: choice of a unique lamp type only slightly subject to variation during transport, substantial increase in the number of lamps in the groups, specification of the manner of excitation and of the duration of prehcating the lamps, ctc. If no progress has becn realized with respect to the dispersion among laboratories, we have, however, observed for cach laboratory a greater consistency than previously between the results of two successive comparisons.

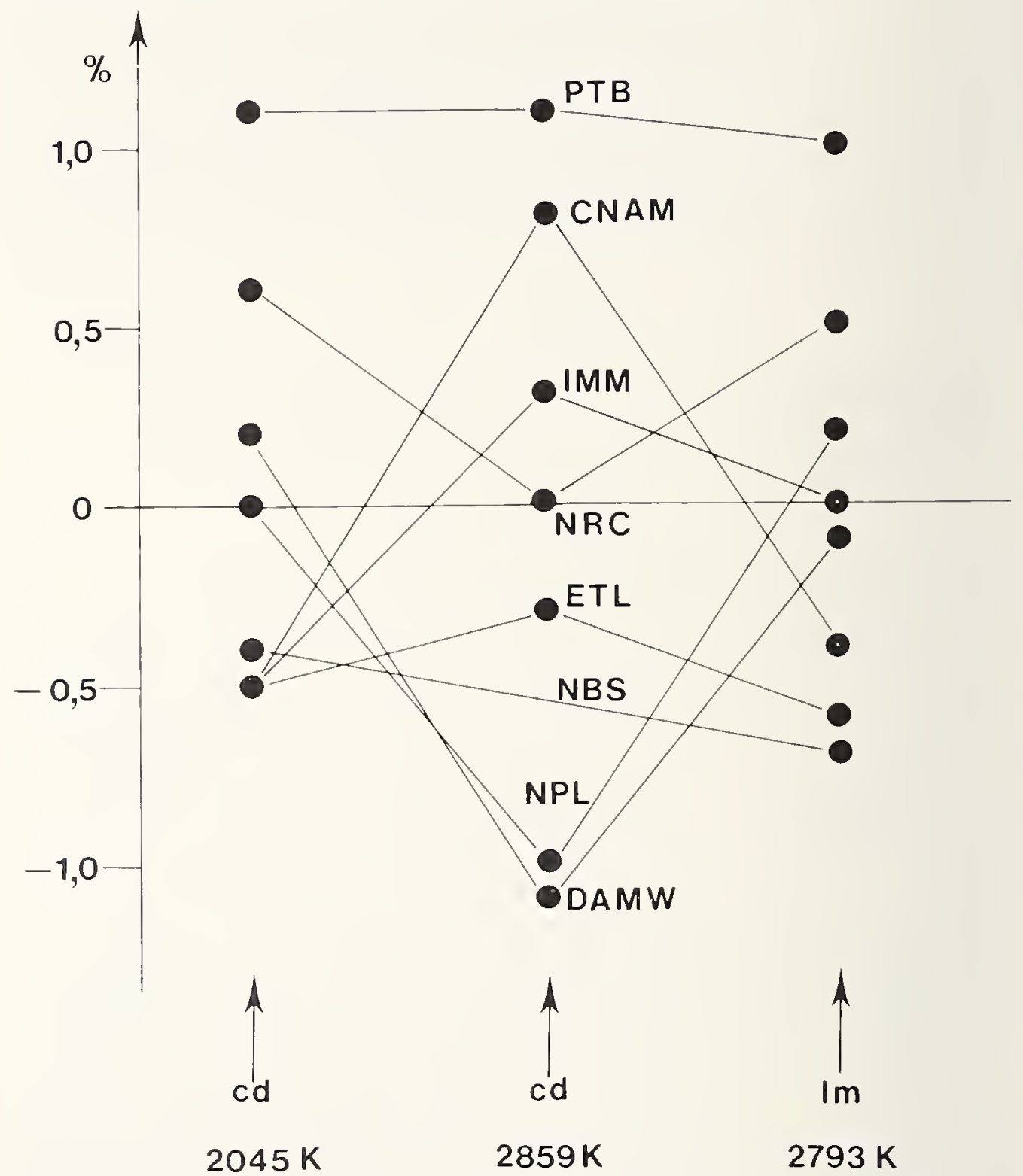

Figure 8. Results of the comparison of national standards of luminous intensity $(2045$ and $2859 \mathrm{~K}$ ) and of luminum flux $(2793 \mathrm{~K}$ ) carried out in 1969 . In the three cases, deviation of each laboratory relative to the mean. (The abloreviations are lhose used by the participatiog laboratories.) 


\section{Maintenance of the Units and Stability of the Standard Lamps}

The BIPM maintains reference standards whose values, in candelas or in lumens, results from the mean of the groups of lamps sent by the national laboratories at the time of the 1952 comparison. These standards are used for the varied work carried out at the BIPM and for furnishing calibrations to laboratories requesting them; several hundred lamps have been sent to Sèvres since 1952 for calibration or for verification of their calibration. The three following international comparisons $(1956,1961,1969)$ have shown that the reference standards of the BIPM have continually satisfactorily represented the mean of the national standards of the laboratories having realized the primary standard at least once.

The uncertainty of the photometric measurements, of the order of 0.5 percent, is such that an attempt at unification of the national realizations of the candela and lumen, made in 1961, failed; it was finally judged preferable to have each laboratory retain the initiative as concerns its own representation of the units. This uncertainty is due essentially to the instabilities of some incandescent lamps; despite a rigorous selection and a preliminary aging with the aim of stabilizing their electrical and photometric characteristics, the lamps remain subject, with almost rare exceptions. both to a change with time which would be easy to take into account, and also, which is more serious, to unexpected variations due to their design or to their construction. Some progress has already been made, in particular for evacuated lamps usable at a low color temperature (fig. 9); but much remains to be done to obtain stable gas-filled lamps, which are, however, the most necessary because they are the most used in practice.

\section{Spectrophotometric Measurements}

The comparison results given above might be interpreted to mean that the realizations of the candela and the lumen at different distribution temperatures are independent units; such is not the case. For example, if the photometric laboratories could obtain radiation sensors whose spectral sensitivity was exactly proportional to $V(\lambda)$, there would evidently no longer be any difficulty in comparing two lamps operated at different distribution temperatures.

There exists, however, a method, which is quite satisfactory theoretically for resolving this delicate problem; it consists of dividing the two radiations to be compared into a very large number of homologous spectral elements and comparing these elements two-by-two, which is a simple photometric measurement; the ratio of the two radiations is then obtained by taking rigorous account of the values of $V(\lambda)$ in the computation. 


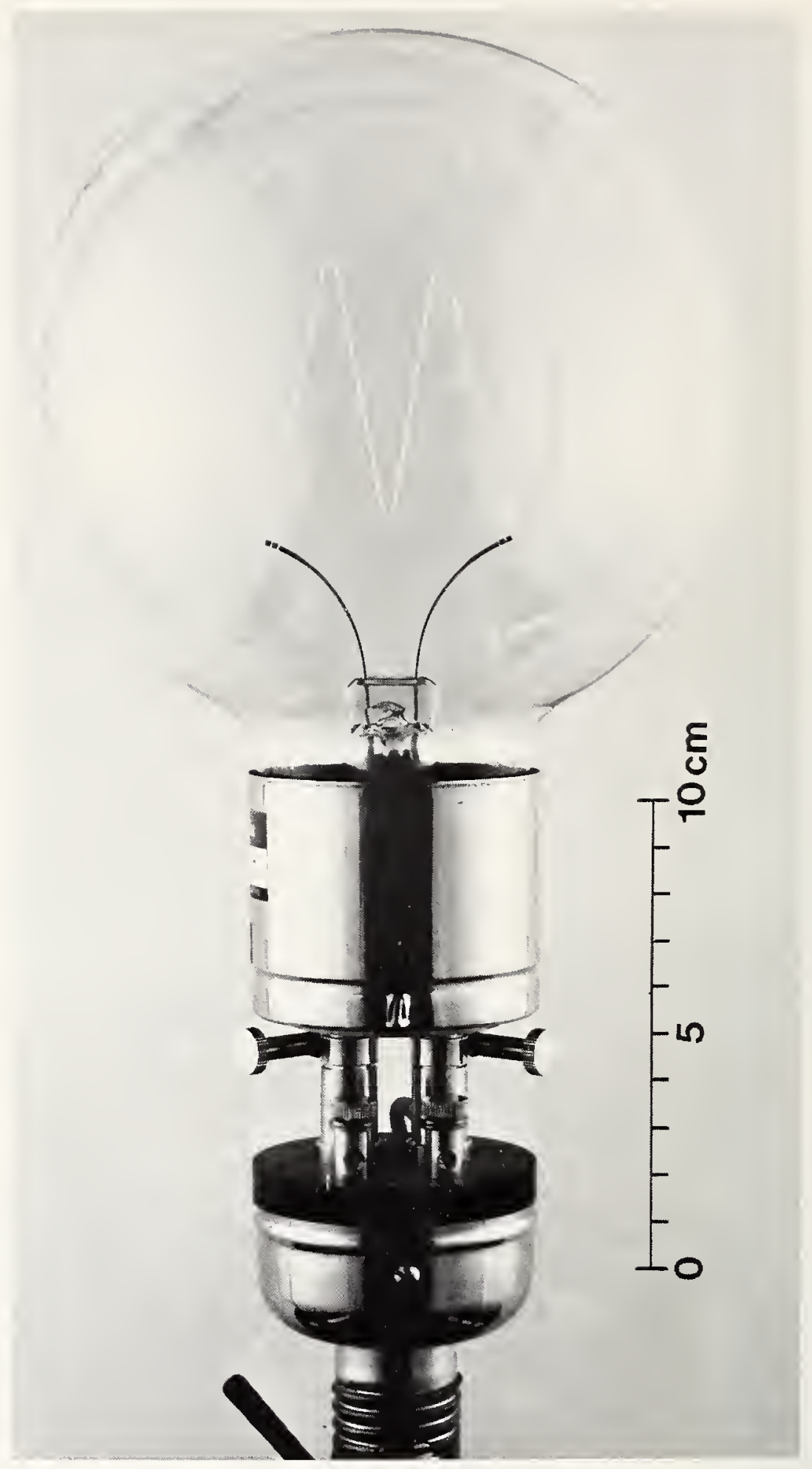

Figure 9. Lamp standard of luminous intensity. Nominal characteristics: 10 V, 5 A, 2045 K, 15 ad: preheat time $15 \mathrm{~min}$. The type of construction (absence of hooks to support the filament), the choice of material (envelope without noticeable defects) and the care taken in assembling the lamp make it a standard of excellent stability. It must, however, never be forgotten that an incandescent standard lamp is a fragile instument. particularly sensitive to - Horch and vibration. 
This method of spectrophotometry, rather long but certainly much more sure, has already been used several times by the BIPM, to see what were the relations which linked the mean representations of the candela at $2045 \mathrm{~K}, 2357 \mathrm{~K}$, and $2859 \mathrm{~K}$; the results obtained show that the magnitude of the candela is the same in the three cases, to better than about 0.3 percent, which can be considered as entirely satisfactory in view of the measurement uncertainties. These results, combined with those of the international comparisons, allow estimating the precision with which the national laboratories compare standards of different spectral distribution temperature; we note some spreads of 1 to 2 percent which show that the methods of heterochromatic photometry used-methods generally based on colored glasses - do not offer the hoped-for reliability of measurement. In the case of the lumen, some older studies lead to similar conclusions.

\section{International Comparison of Distribution Temperature Scales}

We have spoken several times of the distribution temperature without, however, completely specifying which scale is involved. For a very long time, the BIPM has had available only one scale, rather uncertain but nevertheless sufficient for its needs, based on the means of the distribution temperatures near $2045 \mathrm{~K}, 2357 \mathrm{~K}$, and $2793 \mathrm{~K}$, of the national standards of luminous intensity and of luminous flux which were involved in the international comparison of 1952.

In 1963, the organization of the first comparison of standards of distribution temperature enabled one to compare the national scales and establish a mean scale between $2045 \mathrm{~K}$ and $3000 \mathrm{~K}$ [4]. The measurements made by two different methods, one of which was the spectrophotometric method, and at six nominal temperatures distributed rather regularly between 2045 and $3000 \mathrm{~K}$, have shown an appreciable spread among laboratories from $2600 \mathrm{~K}$ up; the maximum spread between the two laboratories whose results are the most divergent reaches several tens of kelvins (fig. 10). The resulting mean scale is a practical scale defined at six points; despite the disagreements among laboratories, and doubtless here also by a happy phenomenon of compensation of errors, one can verify the excellent coherence of the mean scale to a few kelvins.

\section{Prospects Offered by Radiometry}

We see that the work of the BIPM has been restricted until now to the comparison of incandescent lamps, and it is evident that even aside from the realization of the blackbody at the freezing point of platinum, there are some improvements to be made in heterochromatic photometry and in the transfer from standards of luminous intensity to standards of luminous flux. 


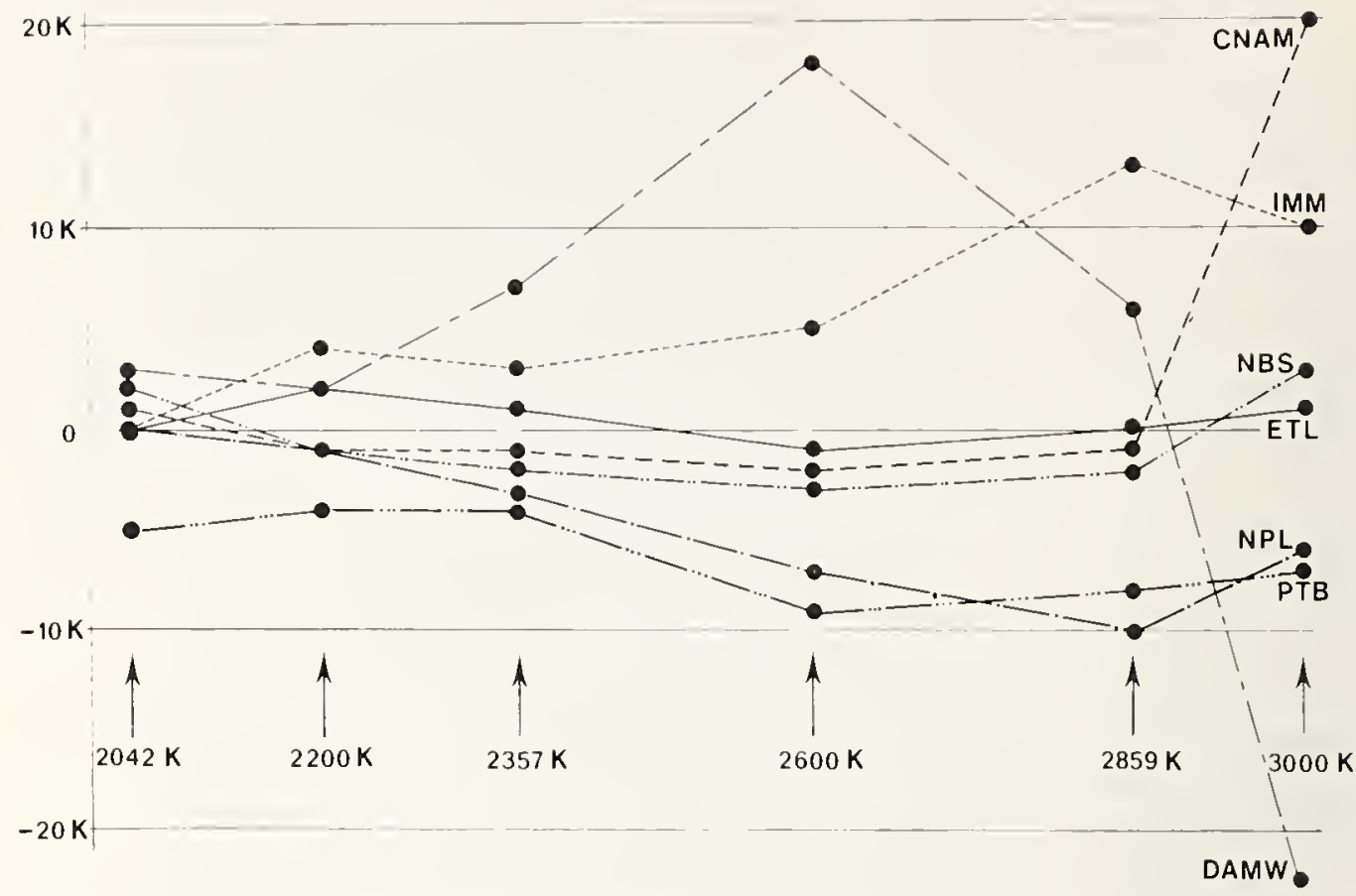

Figure 10. Results of the comparison of national standards of distribution temperature carried out in 1963-1964. At the six nominal temperatures indicated, deviation of each laboratory relative to the mean. (The abbreviations are those used by the participating laboratories.)

But there are also other problems. For example, there exists a disagreement between the calculated value and various experimental values of the maximum luminous spectral efficacy $K_{\mathrm{m}}$, which is the ratio between the luminous flux (in lumens) and the radiant flux (in watts) of a given monochromatic radiation at the wavelength $555 \mathrm{~nm}$ (maximum of the curve $V(\lambda)$ ); the calculated value requires a good knowledge of the freezing temperature of platinum, which has stimulated several national laboratories to undertake new determinations of this temperature. Conversely, one could also, by convention, assign a value to the luminous efficacy of a given monochromatic radiation and realize the photometric quantities by means of an absolute heat detector preceded by a $V(\lambda)$ filter with a known transmission factor; we would then have a new basis for the candela (or for the lumen) which would allow abandoning the blackbody.

Some studies along this line are underway in several national laboratories and some encouraging results have already been obtained; nevertheless, an international comparison of incandescent lamps calibrated in power units, organized in 1966, exhibited the same dispersion among the radiometric scales as among the practical realizations of the candela [5]. It would therefore be premature to change the current definition of the candela. Radiometric measurements, and especially spectroradiometric ones, however, open the way to such applications and their rapid development is inescapable. 


\section{Small Lexicon of Photometry}

\section{Luminous flux}

Quantity derived from energetic flux (energy transported in the form of radiation) by the evaluation of radiation through its action on a selective sensor whose spectral sensitivity is defined by the relative spectral luminous efficacy $V(\lambda)$.

Unit: lumen, $\operatorname{lm}$

Luminous intensity (of a source, in a direction).

Quotient of the luminous flux leaving the source and propagating in an element of solid angle containing the direction, by that element of solid angle.

Illuminance (at a point of a surface).

Quotient of luminous flux received by a surface element containing the point, by the area of that element.

\section{Unit: lux, lx}

Luminance (in a direction, at a point on the surface of a source).

Quotient of the luminous intensity, in the given direction, of a surface element at this point, by the area of the orthogonal projection of that element on a plane perpendicular to the given direction.

\section{Distribution temperature}

Temperature of the blackbody for which the ordinates of the spectral distribution curve of its luminance are proportional, or nearly so, in the visible range, to those of the distribution curve of the radiation considered.

$$
\text { Unit: kelvin, } \mathrm{K}
$$

\section{Candela}

SI unit of luminous intensity: the luminous intensity. in the perpendicular direction, of a surface of $1 / 600000$ square metre of a blackbody at the temperature of freezing platinum under a pressure of 101325 pascals. (13th CGPM, 1967)

$$
\text { Symbol: cd }
$$

\section{Lumen}

SI unit of luminous flux: the luminous flux emitted per unit solid angle (steradian) by a uniform point source having a luminous intensity of 1 candela. (9th CGPM, 1948) 


\section{Bibliography}

[1] Walsh. J.-W. T., Photometry, second edition, 532 pages, Constable and Co., London (1953).

[2] Publications of the Commission Internationale de l'Eclairage No. 18, pp. 43-48 (1970), and No. 15. pp. 93-102(1971).

Procès-Verbaux C.1.P.M. 40, Annexe 1, pp. 145-150(1971).

[3] Terrien, J. and Moreau, H., Rapport sur la dcuxième comparaison des étalons nationaux d'intensité et de flux lumineux (1950-1952), Procès-Verbaux C.1.P.M., 23-B, pp. P76-P100 (1952).

Terrien, J., Moreau, H., and Bonhoure, J., Rapport sur la troisième comparaison des étalons nationaux d'intensitć et de flux lumineux (1956-1957), Procès-Verbaux C.I.P.M. 26-B, pp. P74-P103 (1957).

Bonhoure, J., Rapport sur la quatrième comparaison des étalons nationaux d'intensitć et de flux lumineux (1961), Comité Consultatif de Photométrie, 5 session, pp. 63-87 (1962).

Bonhoure, J., Rapport sur la cinquic̀me comparaison des étalons nationaux d'intensité et de flux lumineux (1969), Comité Consultatif de Photométrie, $7^{e}$ session, pp. P86-P119 (1971).

[4] Bonhoure, J., Rapport sur la première comparaison des étalons nationaux de témperature de répartition (1963-1964), Comité Consultatif de Photométrie, $6^{p}$ session. pp. P47-P65 (1965).

[5] Bonhoure, J.. Comparaison internationale des échelles radiométriques, Rev. Gén. Electricité, 78, pp. 1001-1007 (1969), and Recueil de Travaux du B.I.P.M. 2, article 20 (1968-1970). 


\section{CHAPTER IX}

\section{MEASUREMENT OF RADIOACTIVITY}

\section{Generalities on the Methods of Measurement}

The term radioactivity designates the ability of certain nuclides to emit radiation and particles, and to undergo spontaneous changes. In traversing matter, these radiations lose their energy and produce ions.' electrons, and photons. The measurement of radioactivity is based on the detection of these secondary products: its goal is to determine the average number of nuclear transformations (commonly called disintegrations) per second, that is to say the activity of a source.

We can distinquish two classes of detector depending on whether one counts the primary particles or photons individually (at least in principle). or measures only their average effect. Those of the first class are yenerally called "counters," those of the second can be called detectors. The proportional counter, which has almost completely supplanted the famous CeigerMuiller comter, records the passage of a single ionizin@ particle by multiplication of the primary ions. It belongs to the first class, just as does the scintillation connter which consists of for example, a crystal of sodium iodide in conjunction with a photomultiplier which transforms the scintillations produced in the crystal by the radiation into electrical inupulses, and amplifies these. In contrast, ionization chambers (integrating type) collect without multiplication the charge liberated by the radiation.

A considerable part of the radiation emitted by a source often escapes detection, for example by self-absorption: this prevents a direct measure of activity. Some emitters of more than one radiation, however, under certain conditions lend themselves to an absolute measurement by the method of coincidence counting. An example will be explained in nore detail in the next section. The results obtained are often accurate to a few parts in a thousand.

Let us recall, in passing, that accuracy refers to the difference between the results obtained and the true, but unknown value. On the

\footnotetext{
${ }^{1}$ An ion is an atom or a molecule that is electrically charged. It can be crealed ly the liberation of one or more electrons from a neutral atom or molecule which lies in the path of ionizin" radiation.
} 
other hand, precision characterizes the spread among the results, that is to say, their repeatibility (under conditions as identical as possible) or their reproducibility (under varied conditions). The long-term reproducibility of the results obtained with a well-type ionization chamber can be considerably better than that obtained from other detectors or assemblies: this explains its importance in activity measurements.

For the BIPM, the principal goal is achieving uniformity of measurements throughout the world and over a long time. The only sure means is to improve the accuracy of these measurements: this demands a thorough knowledge of extraneous effects which can introduce systematic errors. Comparisons of independent measurements carried out in different laboratories not only allows checking uniformity but can also sometimes reveal systematic errors. This requires close cooperation with the national laboratories.

Since the comparison of solid radioactive sources presents several inconveniences - source fragility, mountings not adapted to all detectors, impossibility of simultaneous measurement in different laboratories - it is often preferable to compare liquid sources, that is to say, aqueous solutions of radioactive salts, provided that these are homogeneous and stable. One then measures the activity per unit mass of solution, called radioactive concentration. Evidently, this gain in the freedom and generality of the measurement introduces new parameters and additional uncertainties aris ing from the determination of the mass of a drop of solution (sampling) and the preparation of the sources. These techniques and manipulations must be considered as being part of the measurement method. Having determined the mass of the aliquot, one can let the solvent evaporate or add it to another liquid (scintillation liquid).

It is not useful for a small laboratory to try to practice a large number of different methods and techniques. In the case of the BIPM. it has seemed preferable to choose only two or three methods, to study them, and to perfect them as far as possible. The basic method chosen was the $4 \pi \beta(\mathrm{PC})-\gamma$ method of coincidence counting; this method is generally considered as the most accurate for certain radionuclides.

\section{The Method of Counting by $4 \pi \beta(\mathrm{PC})-\gamma$ Coincidences}

The BIPMl has proposed to study this method in its smallest details to increase its precision as much as possible and to consolidate its theoretical bases, in order to be able to apply the various necessary corrections with more certainty. Long term experiments and special studies on the techniques used have already achieved considerable progress.

The method is applicable to radionuclides which, immediately after a $\beta$ disintegration, undergo a transition with the emission of a photon. 
A source is placed inside a proportional counter (PC) which records practically only electrons. irrespective of their direction. and thus has a high efficiency (teometry with a solid angle of $4 \pi \mathrm{sr}$ ). A scintillation comnter using a Nal crystal, placed close to the proportional counter. records only the $\gamma$ rays emitted in a limited solid angle, thus has a reduced, but constant. efficiency. Now these efficiencies can be considered as the probabilities that the corresponding events are recorded. One has to determine only one or the other of these probabilities, banking on the simultaneity of the $\beta$ and $\gamma$ events. An appropriate electronic device counts the number of coincident events.

Let us denote by $\epsilon_{\beta}$ and $\epsilon_{\gamma}$ the respective probabilities that disintenralion of a source atom will be recorded in the $\beta$ channel or the $\gamma$ channel. Then the $\beta-\gamma$ coincidence will be recorded with the probability $\epsilon_{\mathrm{r}}=\epsilon_{\beta} \cdot \epsilon_{\gamma}$, since $\epsilon_{\beta}$ and $\epsilon_{\gamma}$ are independent of each other. The counting rate, that is to say the number of particles. photons. or coincidences recorded per unit time. $\rho_{\beta} \cdot \rho_{\gamma} \cdot \rho_{r}$. observed in the three channels, are all proportional to the sought-for disintegration rate, $\rho_{0}$ :

$$
\begin{gathered}
\rho_{\beta}=\rho_{0} \epsilon_{\beta} \rho \gamma=\rho_{0} \epsilon \gamma . \\
\rho_{c}=\rho_{0} \epsilon_{r}=\rho_{0} \epsilon_{\beta} \epsilon \gamma .
\end{gathered}
$$

whence

$$
\rho_{0}=\rho_{\beta} \rho \gamma / \rho_{c} .
$$

In this simple formula. we have necrlected all loss of counts due, for example. to dearl time' or to inevitable delays in the detectors and the electronics. It is relatively easy to take account of these effects in an approximate way: this seems sufficient in most cases, but there still does not exist a rigorous treatment. The work carried out at the BIPM in this fipld is described later (p. 188).

The BIPM has, moreover, always attached much importance to technical questions on the counting process and source preparation techniques [1.2.3]. Numerous sperial reports. discussions within Section II of the CC.EMRI. and resultiny work, address these questions.

\section{The BIPM Laboratory for Radionuelide Measurements}

A $4 \pi \beta-\gamma$ comntin system, installed in 1962. comprises severah pieces of equipment generously donated to the BIPM by various organizations. Such a system should always be in working order: it is quickly obsolescent and must be updated every 5 years. In addition, a second system was constructed soon after the first, and installed in an air-conditioned room in the

-The minimum interval necessary for recording two separate pulses is called dead time tor resolution time). To establish well defined condirions. an anlificial dead time is oflen imposed. 
new building of the ionizing-radiations section. Four years later. the clectronic system was replaced with a completely transistorized system [4]. Modifications 10 give still more efficient acquisition of ditta are in process. Finure l gives a view of this setup.

Our present technipues for preparing dilutions and sources have been tanght to mumerous foreign guest workers who have come to spend several weeks at the BIP'M. The manipulations of source preparation are illustrated by figure 2 .

Let us recalt finally that the BIPM has prepared and standardized nearly 10000 sonces since 1963: several hundred of these have been placed at the disposal of various national laboratories.

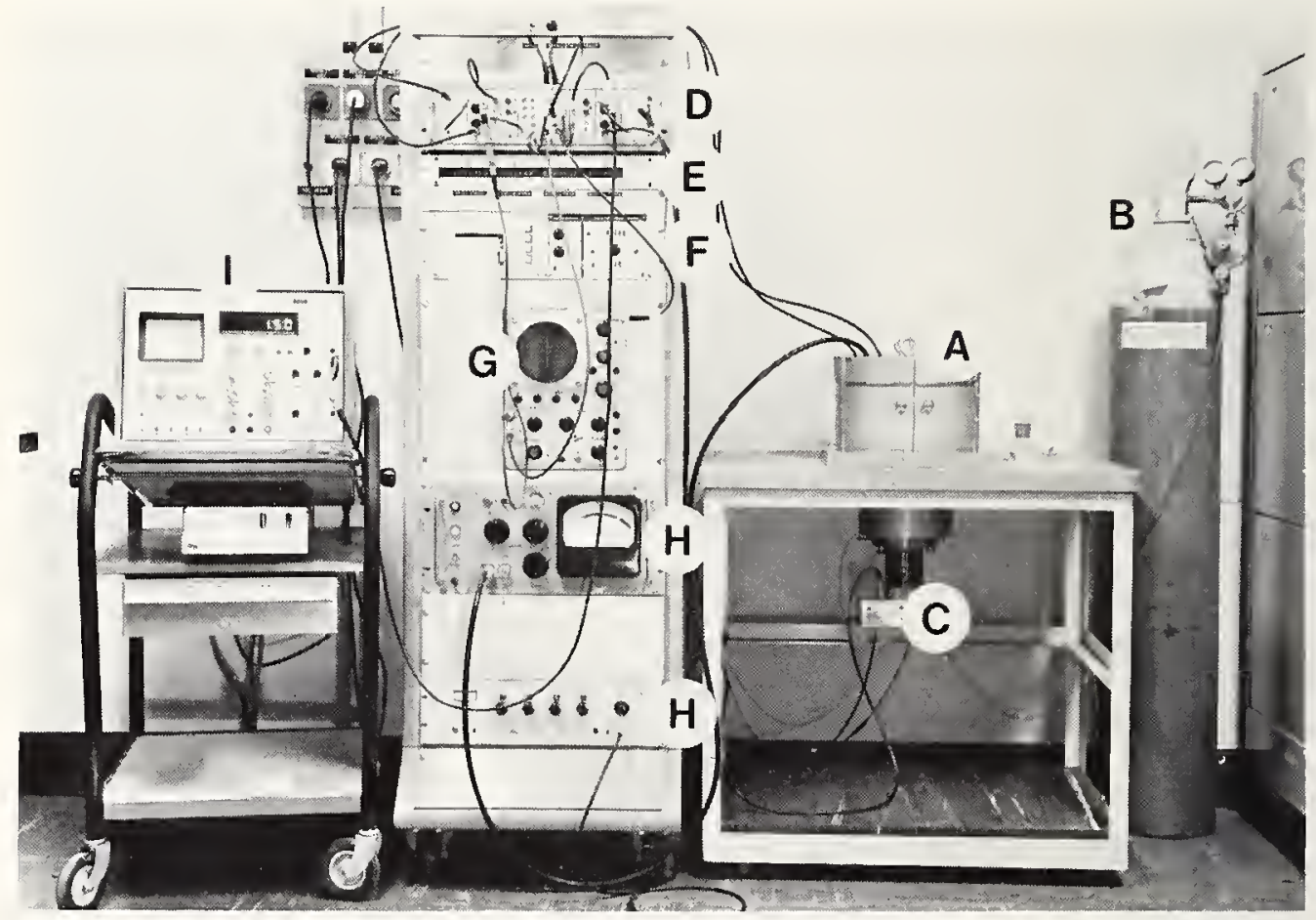

Fiqure 1. View of the BIPl system for counting by the $4 \pi \beta$ P C $-\gamma$ coincidence method. A: lead rastle containing the detectors and the soure to be measured: B: bothle containing was which circulates thromels the proportional counter: C: peamplifier of the scintillation counter (for y radiation): D: transistorized electronic system: E: counter displays: F:

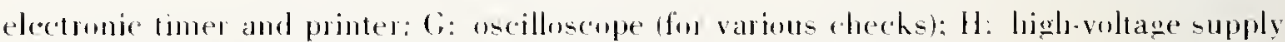
for the detectors: I: multichannel andyzer ifor recording $\gamma$ spectra). 


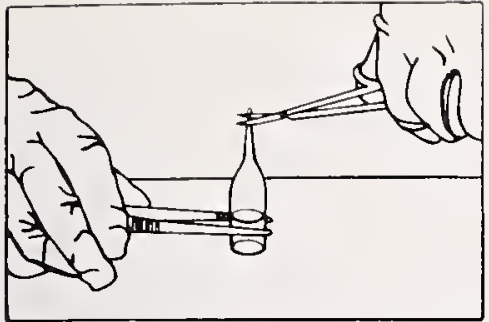

a

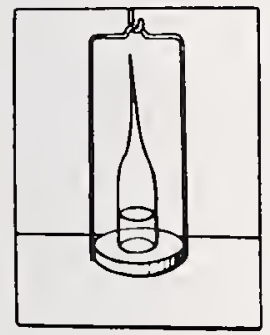

d

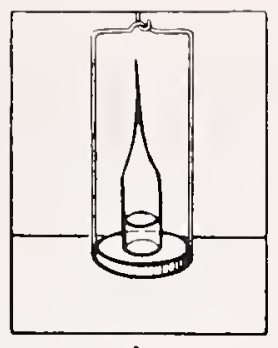

b

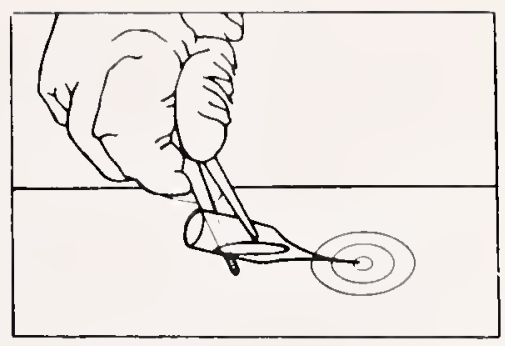

C

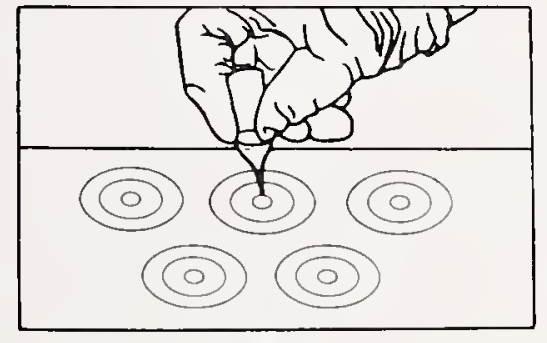

e

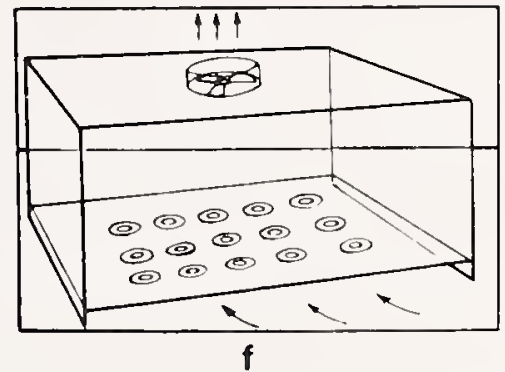

Figure 2. Preparation of radioactive sources. Manipulations are carried out with gloves to avoid contaminating the hands. a: The polyethylene pycnometer containing rachoactive solution; the end seal of the drawn-out neck is being cut. b: First weighing of the pycnometer on the platform of a microbalance. The pycnometer $(\approx 1 \mathrm{~g})$ contains 1 to $2 \mathrm{~g}$ of solution. c: Deposition of several drops ( $50 \mathrm{mg}$, on the average) on the support. This latter is a film of thin plastic. metallized and stretched across the hole of a metallic washer. d: Second weighing of the pycnometer; the mass of solution used is obtained by the difference from the preceding weighing. e: A drop of wetting agent is added to each source with the aid of another pycnometer. $f$ : Drying the sources in a current of air. The cleanliness of the instruments must be carefully guarded.

\section{Radioactivity Standards}

More than 1800 nuclides are known today; most of these are radioactive. The measured half-lives ${ }^{3}$ range from less than a microsecond to more than a billion years. The radionuclides of metrological interest have halflives between an hour and several thousand years. For the choice of a standard, it is important to recall that the results of a measurement of radioactive decay of, in general, a mixture of radionuclides depend on the values of the half-lives of each radionuclide present, and on the mixture ratios of the radionuclides. These ratios are not known with certitude; the useful life of a standard is therefore also not known. A practical solution consists of preparing a batch of standards and replacing it with a new batch well before the sources become too weak.

In principle, the unit of activity could be maintained with the help of a single absolute measurement device, without use of standard sources. It has been established, however, that a $4 \pi \beta(\mathrm{PC})-\gamma$ system, probably the device best adapted to this use, can remain unchanged for only a few years.

${ }^{3}$ The radioactive half-life is the time required for the activity to decrease to half its initial value. 
It is therefore safer to refer also. if possible. to one or more appropriate standard sources. At the BIPM we have chosen a certain number of " ${ }^{\prime \prime} \mathrm{Co}$ and ${ }^{5-1}$ In sources, having respective half-lives of 1926 and 312 days; in the course of measurements. we slip these in among the sources whose activity is to be determined. Each standard can be used for one or two half-lives and then must be replaced by a new source. This procedure guarantees grood continuity of measurements while furnishing absolute results. It has the inconvenience of being valid only for the radionuclides mentioned and requires a rather delicate source preparation.

In certain cases. we prefer to sacrifice the absolute character of the method. and thus the measurement accuracy. for the benefit of better precision. This is the case for current-measuring ionization chambers like the well chamber (where one has provided a tube, or well, for placing the source inside the chamber). Such a chamber, also called a $4 \pi \gamma$ chamber. can constitute a reference device of very high reliability for $\gamma$ emitting radionuclides if the current measurements are made relative to an appropriate radium source (half-life $\approx 1600$ years).

The BIPM has embarked on a permanent reference system for international comparisons. This system comprises a $4 \pi \gamma$ chamber, an automatic system for current measurement, and a set of special radium sources.

It is expected that the national laboratories that distribute radioactive solutions will send some of their production ampoules to the BIPM. We shall measure them. store them. and communicate the complete results, thus establishing the possibility of relating these standards and achieving uniformity of activity measurements on $\gamma$ emitters.

The international radium standard. stored at the BIPM (see p. 37), no longer has great metrological importance aside from its historical-interest and its well defined mass of very pure radium. The mobility of the salt in its container diminishes the validity of ionization measurements, and the increasing fragility of the container forbids frequent use.

\section{International Comparisons of Radionuclides}

We estimate that we can measure the activity of certain radionuclide sonrces, such as ${ }^{60} \mathrm{Co}$. to a few parts in a thousand. This uncertaint y is composed of a random part and a systematic part. The first can be determined by the laws of statistics: it decreases when the number of independent measurements increases. The second. however, is determined only by an estimate whose validity depends on the experience of the operator and the extent of possible variations of parameters (method, operator, laboratory. measurement conditions. etc.).

The systematic error can be estimated on firm crrounds only if comparative measurements are carried out between various laboratories and by different methods. The CCEMRI has recognized this need since the 
beginning of its existence. Numerous comparisons of radionuclides had already been made before, but it was considered desirable that they be continued on a more extensive and official basis. The goal, which might seem ambitious. was formulated by the CCEMRI in 1961: "It is desirable that an accuracy of $\pm 1 \%$ - possibly going to $\pm 0.1 \%$ in certain cases - be attaincd in the standardization of radionuclides."

Comparisons organized by the BIPM began in 1961 at a very rapid pace but this subsequently slowed down (see table 1). As each of these comparisons has been described in detail [5], we limit ourselves to presenting here some interesting figures.

Part of the results of the international comparison of ${ }^{\prime \prime} \mathrm{Co}$ in March 1963 is represented graphically in figure 3 . One will note the small difference (statistically significant) linked to the method of weighing drops of solution. Several authors have subsequently very carefully studied this difference which would have perhaps passed unnoticed if the comparison had not revealed it.

The temporary halt of comparisons after 1967 was due to the predominant opinion of the participants who found that the results, often difficult to interpret, did not always justify the difficult work since a certain number of problems had not been studied [6]. These problems have been distributed among several working groups of which one is rightly charged with preparing new proposals for international comparisons.

\section{Statistics of Counting}

All the tasks undertaken in this field at the BIPM have their origin in a practical goal. In certain cases, it is a matter of finding, adapting, or developing methods which permit the extraction of maxinum useful information from the results of measurements which have been made with the greatest possible care. In other cases, one is led to the use of statistical reasoning to more correctly describe an observed process, as, for example, the counting of pulses of nuclear origin whose distribution departs appreciably from a simple Poisson Law. ${ }^{4}$ To neglect these deviations or to take account of them only in an imperfect way would inevitably introduce systematic errors. Finally, such a description can sometimes give rise to a new measurement method.

Here are some examples of applications in different fields which have been treated at the BIPM:

- Exploration of experimental data: analysis permitting discovery of distortions due to a dead time, method of extrapolation of the profile of a spectral line of $\alpha$ particles to zcro intensity, smoothing an experimental spectrum.

\footnotetext{
${ }^{4}$ In this case, the probability of observing $n$ pulses in a time interval $t$ is given by e ${ }^{-\rho t}(\rho t)^{n} / n !$. where $\rho$ is the counting rate (mean number of pulses per unit time).
} 
$B C M N$

CENS

IKO

IMM

ISS

JEN

PTB

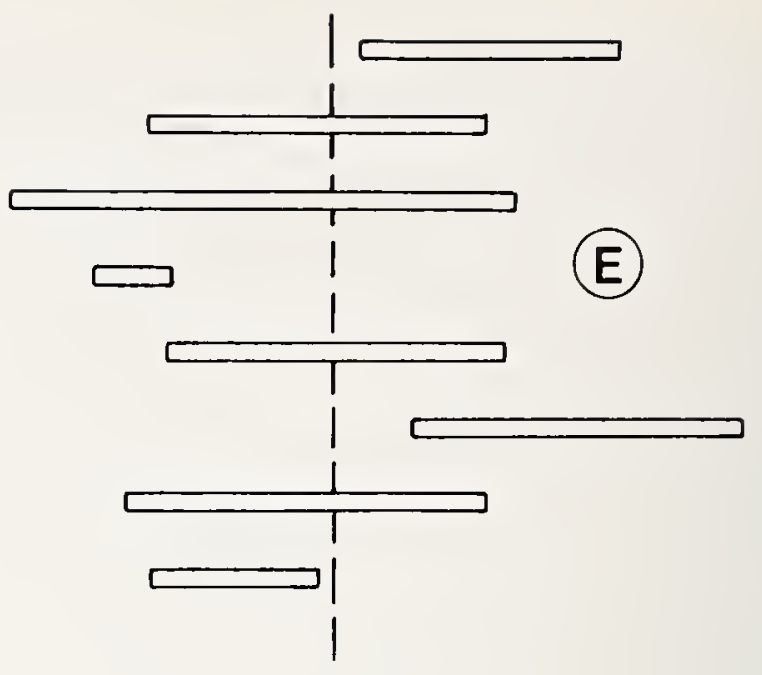

$A E C L$

AIEA

BIPM

CNAM

ETL

ISN

NBS

NPL
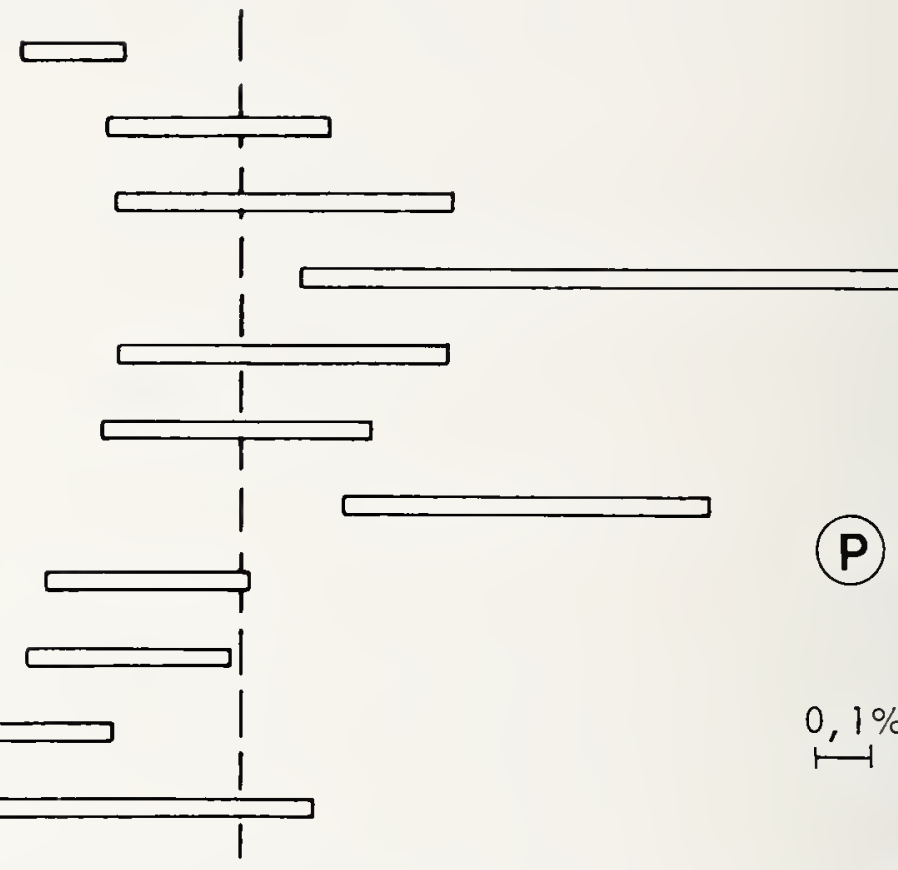

NPRL

NRC

UVVVR

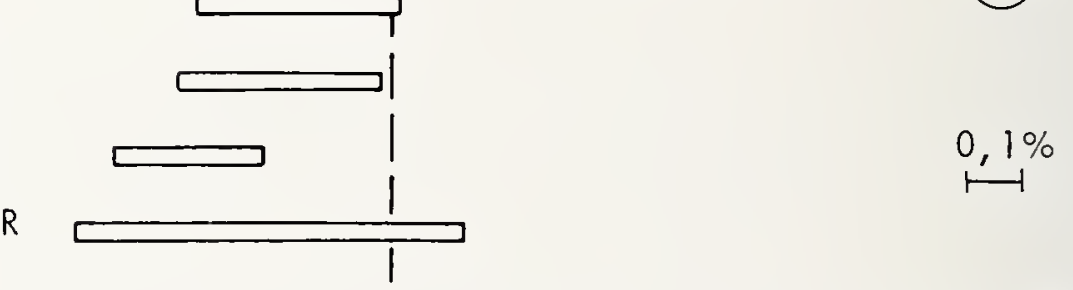

1510

1520

Figure 3. International comparison of a solution of ${ }^{60} \mathrm{Co}$ (May 1963). The horizontal bars represent mean results (activity per gram) with their standard deviations. The vertical lines (broken) indicate arithmetic means of groups of results obtained either by the pycnometer method (P), or by the "extrapolation" method (E) in which the deposited drops are weighed directly. but in which allowance must be made for evaporation before and during the weighing, by extrapolation to the initial instant. The initials at the left designate the participating laboratories. 
Table 1. Renume of the eleven international comparions of radionuclides organized lyy the BIPNI

\begin{tabular}{l|l|c|c|c|c}
\hline Radionuclide & Date & $\begin{array}{c}\text { Number of } \\
\text { participants }\end{array}$ & $\begin{array}{c}\text { Total } \\
\text { range } \\
(\%)\end{array}$ & $\begin{array}{c}\text { Number of } \\
\text { selected } \\
\text { results }\end{array}$ & $\begin{array}{c}\text { Range of } \\
\text { the selected } \\
\text { results } \\
(\%)\end{array}$ \\
\hline${ }^{32 P}$ & Jan. 1961 & 16 & 6.0 & 13 & 2.0 \\
${ }^{{ }^{311}}$ & April 1961 & 16 & 4.0 & 12 & 1.5 \\
${ }^{198} \mathrm{Au}$ & Jan. 1962 & 25 & 3.1 & 15 & 2.5 \\
${ }^{60} \mathrm{Co}$ & Jan. 1962 & 21 & 9.0 & 18 & 3.0 \\
${ }^{204} \mathrm{Tl}$ & May 1962 & 19 & 14 & 14 & 5.9 \\
${ }^{35} \mathrm{~S}$ & June 1962 & 15 & 18 & 4 & 6.5 \\
${ }^{60} \mathrm{Co}$ & March 1963 & 22 & 4.6 & 19 & 1.4 \\
${ }^{241} \mathrm{Am}$ & June 1963 & 21 & 3.1 & 13 & 0.8 \\
${ }^{90}(\mathrm{Sr}+\mathrm{Y})$ & Feb. 1964 & 23 & 3.0 & 16 & 2.0 \\
${ }^{54} \mathrm{Mn}$ & Apr. 1965 & 16 & 14 & 15 & 6.0 \\
${ }^{60} \mathrm{Co}$ & May 1967 & 24 & 1.7 & 20 & 0.9 \\
\hline
\end{tabular}

- Process description: superposition of regenerative processes, ${ }^{5}$ time intervals between pulses after an extendable dead time (fig. 4), modifications of a Poisson process by a nonextendable dead time, effect of two successive dead times, statistics of pairs of pulses, time intervals between correlated pulses.

- New methods: determination of dead time to better than 0.1 percent, coincidence counting by a delay method, measurement of secondary pulses.

Most of the problems concerning dead time have been described in two articles which give a comprehensive view of the current state of our knowledge in this area $[7,8]$. Other studies have been treated in internal reports of the BIPM; these are available on request.

The majority of these studies aim at a better knowledge of the corrections to be applied to counts obtained by the $4 \pi \beta-\gamma$ method. The study of perturbations capable of causing systematic errors is a permanent task. Numerous problems are not yet resolved in a satisfactory way, as, for example, the loss of coincidences produced by the dead times in the $\beta$ and $\gamma$ channels. At the same time, these already well-advanced studies are being actively pursued, with the aim of a simplified application of the correlation method. This method is a new route which may rival the direct measurement of coincidences.

To illustrate the results obtained by these studies, we have chosen the two following examples which outline some new measurement methods that have already aroused lively interest in other laboratories.

\footnotetext{
${ }^{5}$ In a regenerative process, successive events occur independently of each other. Such a process is thus completely characterized by the distribution function of the time intervals between successive events.
} 

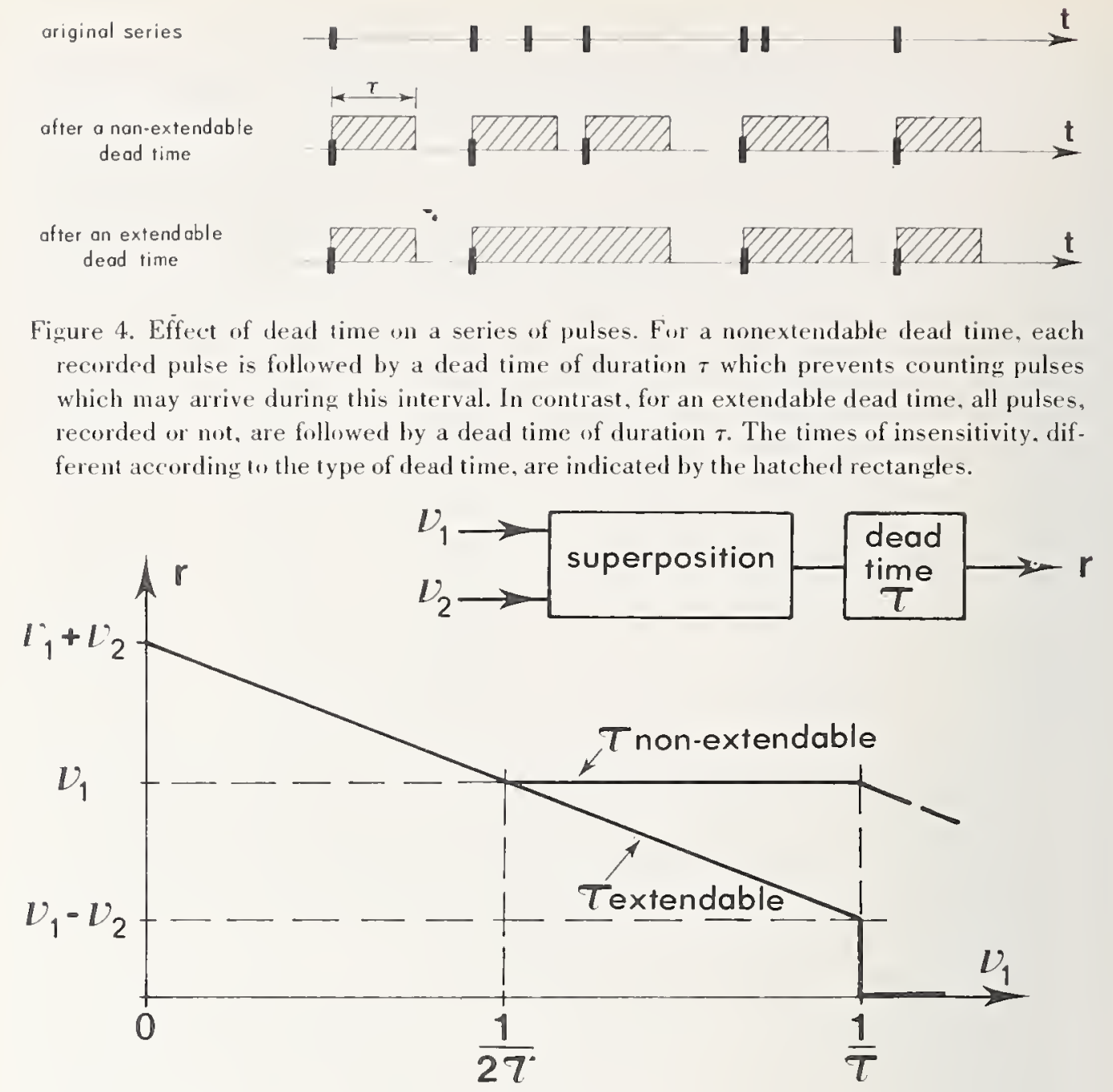

Figure 5. Precise measurement of an imposed dead time.

- upper: scheme of the principle for measuring $\tau$ with the use of two oscillators.

- lower: counting rate $r$ measured as a function of the frequency $\nu_{1} ; \nu_{2} / \nu_{1}$ is assumed constant.

\section{a) Precise measurement of dead time}

If the elaboration of more and more rigorous correction formulas for lost counts is indispensable, it is important that the numerical values inserted into these formulas be sufficiently accurate, in particular the duration of the dead time. Traditional techniques (two-source method, decay method) all have the inconvenience of being tedious and of low precision. Furthermore, they assume that the original process follows a Poisson Law strictly and that the type of dead time is known in advance. Now to measure a dead time, we are not obliged to use a series of pulses coming from a natural source; it is only important to know the distribution of time intervals between successive events. This distribution is particularly simple if we use a superposition of pulses coming from two oscillators of frequencies $\nu_{1}$ and $\nu_{2}$ (with $\nu_{1}>\nu_{2}$ ). Figure 5 illustrates the results obtained for the 
counting rate $r$ of the superposed process. It is assumed that the length of the pulse is negligible with respect to the dead time $\tau$. For the range $\nu_{1}<$ $1 / 2 \tau, r$ is independent of the type of dead time. $\tau$ can be deduced from the relation

$$
\tau=\left(\nu_{1}+\nu_{2}-r\right) / 2 \nu_{1} \nu_{2}
$$

On the other hand, for $\nu_{1}>1 / 2 \tau$, the counting rate $r$ depends on the type of dead time and thus permits its identification.

\section{b) Measurement of secondary pulses}

Questions concerning the detection of secondary pulses hold a particular interest for all absolute measurements of activity, for the occasional occurrence of more than one pulse for a single initial event risks a systematic falsification of the count. Such duplications can show up by their influence on the distribution of intervals between successive pulses. Other perturbations of the process under study have, however, a similar effect, and if an anomaly is observed it is often difficult to attribute it to the phenomenon of double-pulsing with certainty. For a series of pulses containing simple events which occur individually and thus which appear in the form of pairs, the total number of observed counts per time interval $t$ can be decomposed into $k=k_{1}+2 k_{2}$, where $k_{1}$ and $k_{2}$ are respectively the number of simple pulses and the number of pairs in $t$. We note that $k$ and $k_{1}$ are both even or both odd, whatever $k_{2}$ is. Analogously we can write for the total counting rate, $\rho^{\text {tot }}=\rho_{1}+2 \rho_{2}$. This property is the basis of a statistical method which allows resolution of the contributions $\rho_{1}$ and $\rho_{2}$ to the total counting rate.

The pulses are directed to an electronic flip-flop which changes state each time a pulse arrives; the same pulses are also sent to a second flipflop, but after a delay $t$ during which $k$ pulses reach the first flip-flop. Each flip-flop controls a gate which opens and closes alternatively after each pulse. The two gates are initially in the same state: they find themselves again in the same state if $k$ is even and in different states if $k$ is odd. Examination of the state of these gates allows determination of the probabilities Prob ( $k$ even) and Prob ( $k$ odd). We also know how to calculate these probabilities in the case where the distribution of separation follows a Poisson law; the probably that $k$ is odd is independent of the number of pairs of pulses and is given by

$$
\operatorname{Prob}(k \text { odd })=1 / 2\left[1-\exp \left(-2 \pi_{1} t\right)\right] .
$$

Since Prob ( $k$ odd) is measured, this relation allows the computation of $\rho_{1}$. Moreover, one obtains $\rho_{\text {tot }}$ by direct counting, and calculates

$$
\rho_{2}=1 / 2\left(\rho_{\text {tot }}-\rho_{1}\right) \text {. }
$$

If the delay $t$ is much less than the mean interval $\delta$ between the pulses forming a pair, only a single one of the two pulses of a pair can appear in 
this interval, in general. On the contrary, if $t$ is much larger than $\delta$, the two pulses of a pair practically always appear in the same interval. Figure 6 shows that these limiting behaviors are independent of the distribution of the interval between pulses of a pair.

This reasoning has been fully confirmed by experiment. Even for a ratio so small that $\rho_{2} / \rho_{\text {tot }}=0.005$, the secondary pulses show up clearly and can still be measured.

Some details on this new type of measurement and a generalization of the method in the case of multiple secondary pulses are found in the references [9-11].

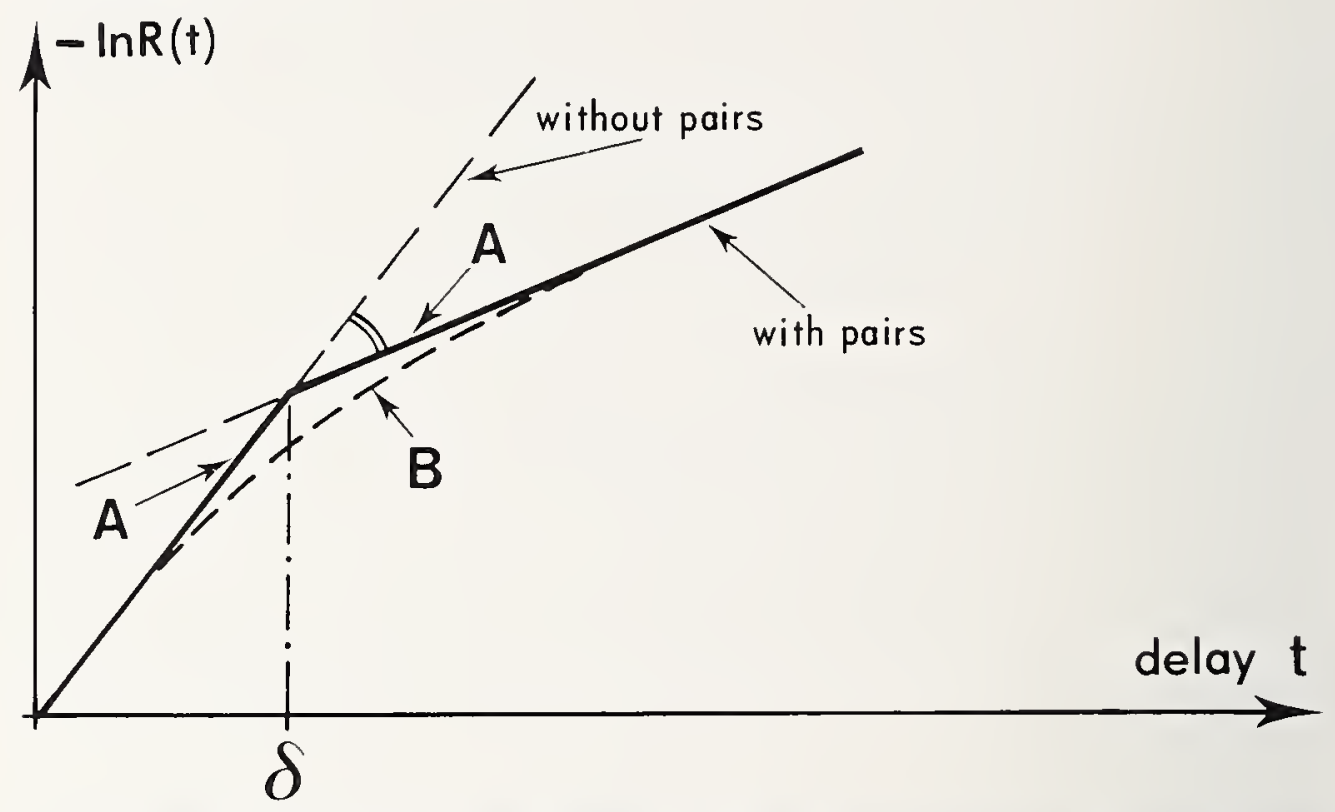

Figure 6. Measurement of secondary pulses. Graphical representation of - In $R(t)$, where $R(t)=1-2$ Prob ( $k$ odd), for a distribution which is originally Poisson. The two straight lines represent the behavior limits: the difference of their slopes is proportional to the counting rate $\rho_{2}$ of paired pulses. The intervals between pulses of a pair are assumed equal to $\delta$ (case $A$ ) or distributed about the mean value $\delta$ according to an experimental law (case B).

\section{Energy Measurement: Alpha-Particle Spectrometry}

In 1965, it was decided that the BIPM would engage in the field of energy measurement of $\alpha, \beta$, and $\gamma$ rays to be able to express an official opinion on the energy of certain lines. It was first occupied with $\alpha$ spectrometry, where the need for unification was urgent and where it could benefit from certain previous experience.

Nearly $300 \alpha$-emitting radionuclides are known; this is slightly greater than the number of stable nuclides. The $\alpha$ particle is a helium nucleus with electric charge $+2 \mathrm{e}$, comprising two protons and two neutrons. For each $\alpha$ emitter these particles are ejected with one or more discrete energy values. The excellent definition of these energies, normally between 4 and $9 \mathrm{MeV}$, gives them the quality of standards. 
The preferred method for measuring absolute energy is that of deflection by a uniform magnetic field. Knowing that a charged point mass having a velocity perpendicular to the direction of the field describes a perfect circle in vacuo, one measures the magnetic flux density $B$ and the radius $r$ of this circle. The kinetic enrgy $E_{\alpha}$ is then, except for a relativistic correction,

$$
E_{\alpha}=(2 e / m)(B r)^{2},
$$

where $2 e$ and $m$ are respectively the charge and mass of the $\alpha$ particle.

The magnetic spectrograph of the BIPM (fig. 7) is the only one in the world which has been specially designed for the absolute measurement of the energy of $\alpha$ particles. The electromagnet of $8.5 \mathrm{Mg}$ has a semicircular airgap of $650 \mathrm{~mm}$ radius and $70 \mathrm{~mm}$ spacing. The flux density, stabilized to about $1.5 \times 10^{-6}$ with the help of a proton-resonance probe, can have several values between 0.6 and $1.0 \mathrm{~T}$. Into the spacious vacuum chamber, one places either the device for measuring magnetic flux density, or the spectrograph proper; this latter essentially comprises a slit and a photographic plate held in the same plane at a fixed distance apart by a rigid bar. The photographie plate serves as an $\alpha$-particle detector. The principle of the functioning of the semicircular spectrograph is indicated in figure 8 .

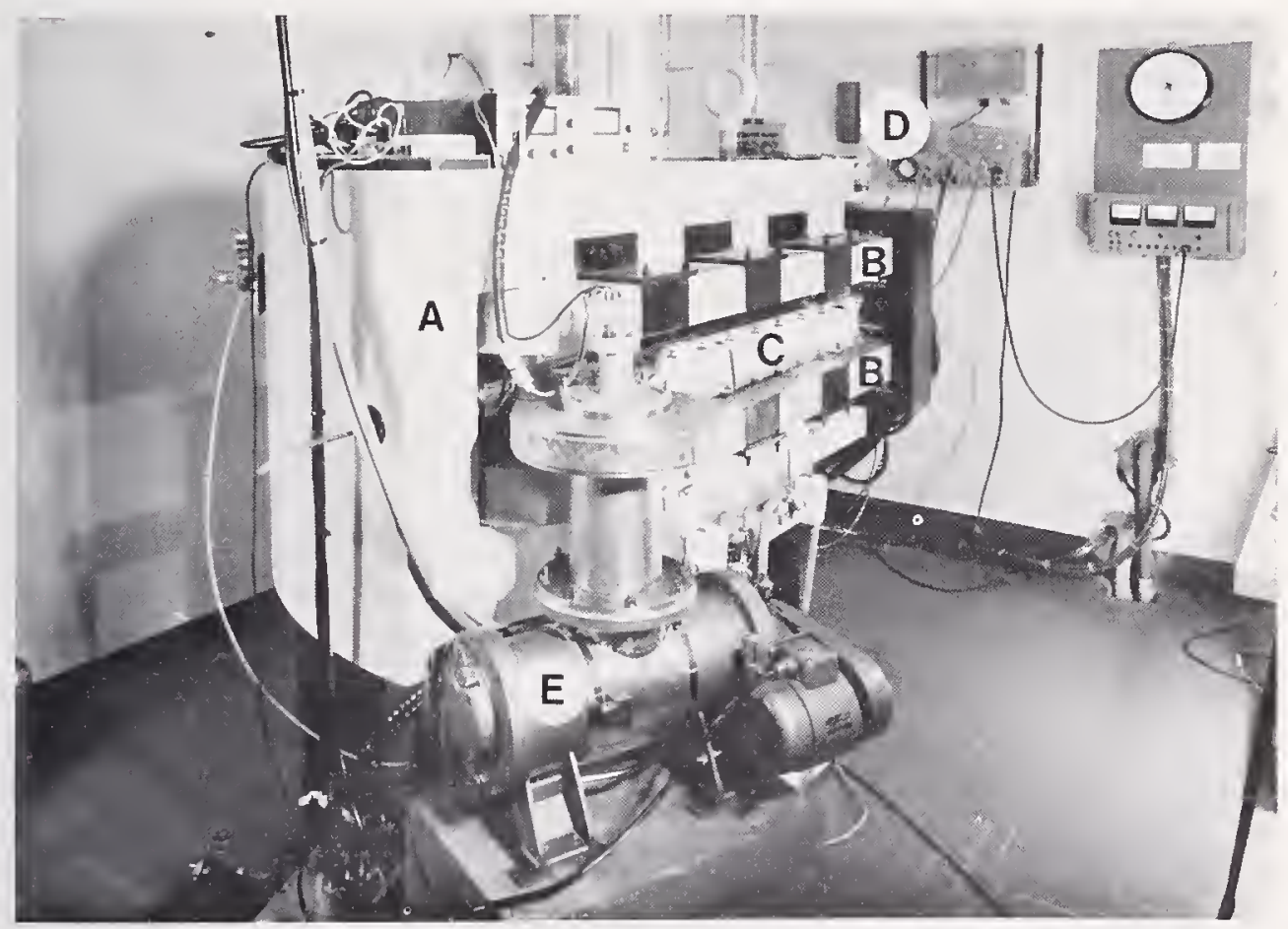

Figure 7. Overall view of the BIPM magnetic spectrograph for absolute measurement of the energy of $\alpha$ particles. A: electromagnet (mass $8.5 \mathrm{Mg}$ ), maximum flux density $1 \mathrm{~T}$; $\mathrm{B}$ : water-cooled coils: $C$ : cover of the vacuum chamber; D: device for stabilizing the magnetic field using proton resonance; E: turbomolecular vacuum pump (speed: $900 \mathrm{~m}^{3} \mathrm{~h}^{-1}$ ). 


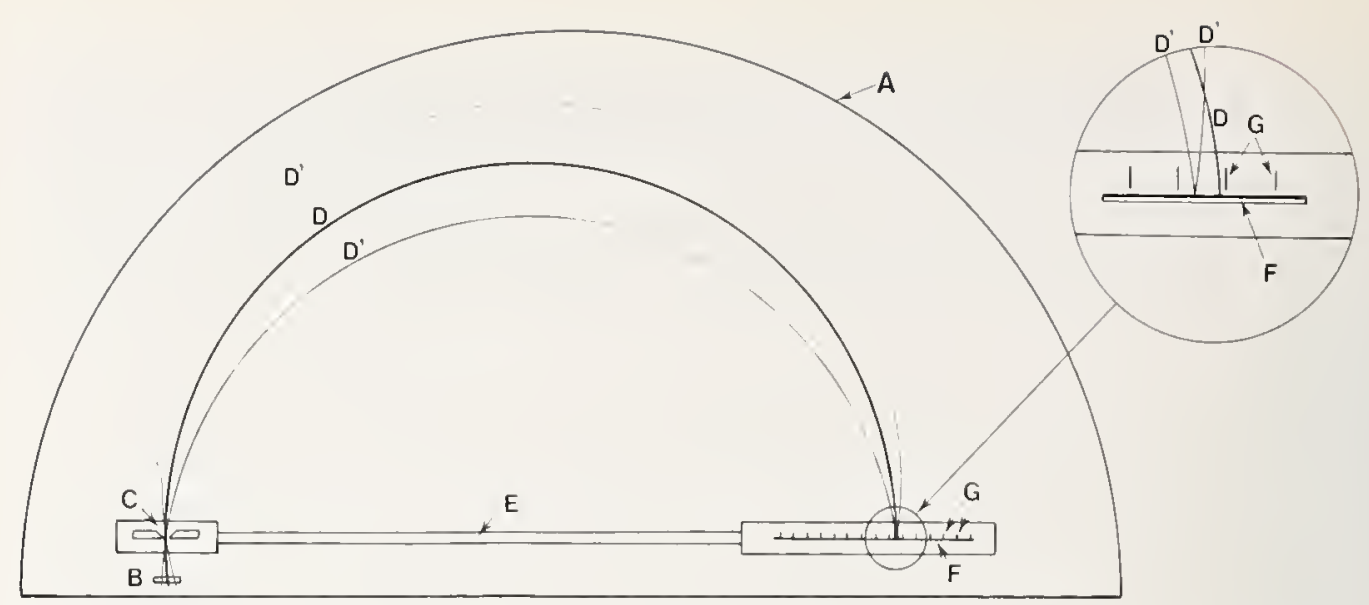

Figure 8. Diagram showing the principle of the semicircular spectrograph. Under the action of the uniform magnetic field perpendicular to the plane of the figure, $\alpha$ particles of the same energy follow circular paths of the same radius $r$. A: pole face of the electromagnet; B: $\alpha$-particle source; C: slit: D.D': $\alpha$-particle paths: the particles enitted perpendicularly to the direction CF (path D) give the limit of the recorded line: E: rigid bar to maintain the distance from $C$ to $G$; F: detector (photographic plate): $G$ : reference scale whose distance from $C$ has been previously measured. The scale is recorded on the photographic plate at the same time as the $\alpha$-particles.

The distance between the slit and the detector impact point of the particle emitted by the source depends upon the energy of the particle and on its direction of passage through the slit. To a group of monoenergetic particles emitted by a source of limited size, there corresponds a calculable distribution of surface density of the number of particles on the detector (fig. 9). Since sources are not infinitely thin, the energy of a group of particles spreads, because of self-absorption, over a certain range which is called a "line"; its upper limit does not depend on the source thickness. This limit can be determined by extrapolating to zero the density of tracks on the plate. Experience confirms that this density increases as the $3 / 2$ power of the distance from the extrapolated point. Consequently, if the density is plotted to the $2 / 3$ power on a graph, the observed values lie on a straight line, except for statistical uncertainties. By extending this line to its intersection with the baseline (fig. 10), one obtains the extrapolated point whose distance from the inner edge of the slit is equal to the diameter $2 r$. The baseline, always present, comprises tracks whose origin is not in the source. Details of the method and of the experimental device are described in the reference [12].

At the time of writing, 37 different lines from 24 emitters have been measured with uncertainties varying between $10^{-5}$ and $10^{-4}$. These new results have permitted adjusting a large number of earlier measurements and publishing a complete catalog of recommended $\alpha$-particle energy values [13]. It must be noted, moreover, that starting from the energy of an 
$\alpha$ particle and recoil nucleus, one can calculate the energy of disintegration which, in turn, is equivalent to the difference between the mass of the emitting atom and the sum of masses of the residual atom and the $\alpha$ particle. The systematics of atomic masses makes large use of the new values of $\alpha$-particle energies determined at the BIPM.

Despite the limited number of $\alpha$ emitters which can be measured, these experiments are far from being finished. Problems relating to the state of the sources and to the natural widths of the lines have not yet really been touched, but will merit special attention.

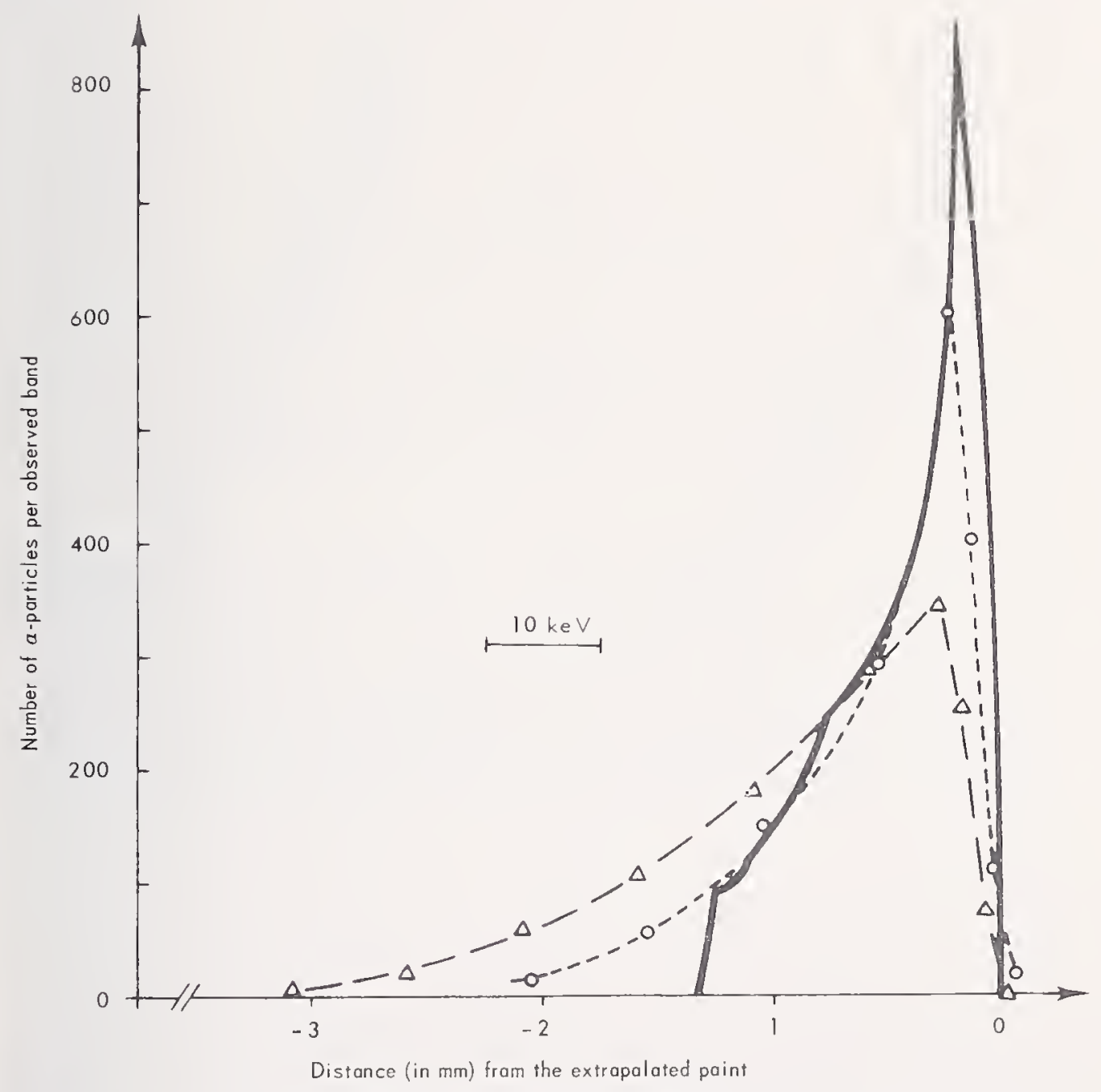

Figure 9. Line profiles obtained by semicircular spectrography. The $\alpha$-particle tracks have been counted in $0.02 \times 5 \mathrm{~mm}$ bands on the photographic plate as a function of distance from the slit (fig. 8). $\Delta$ measured profile, thick source: O measured profile, thin source; - profile calculated for monoenergetic $\alpha$ particles. 


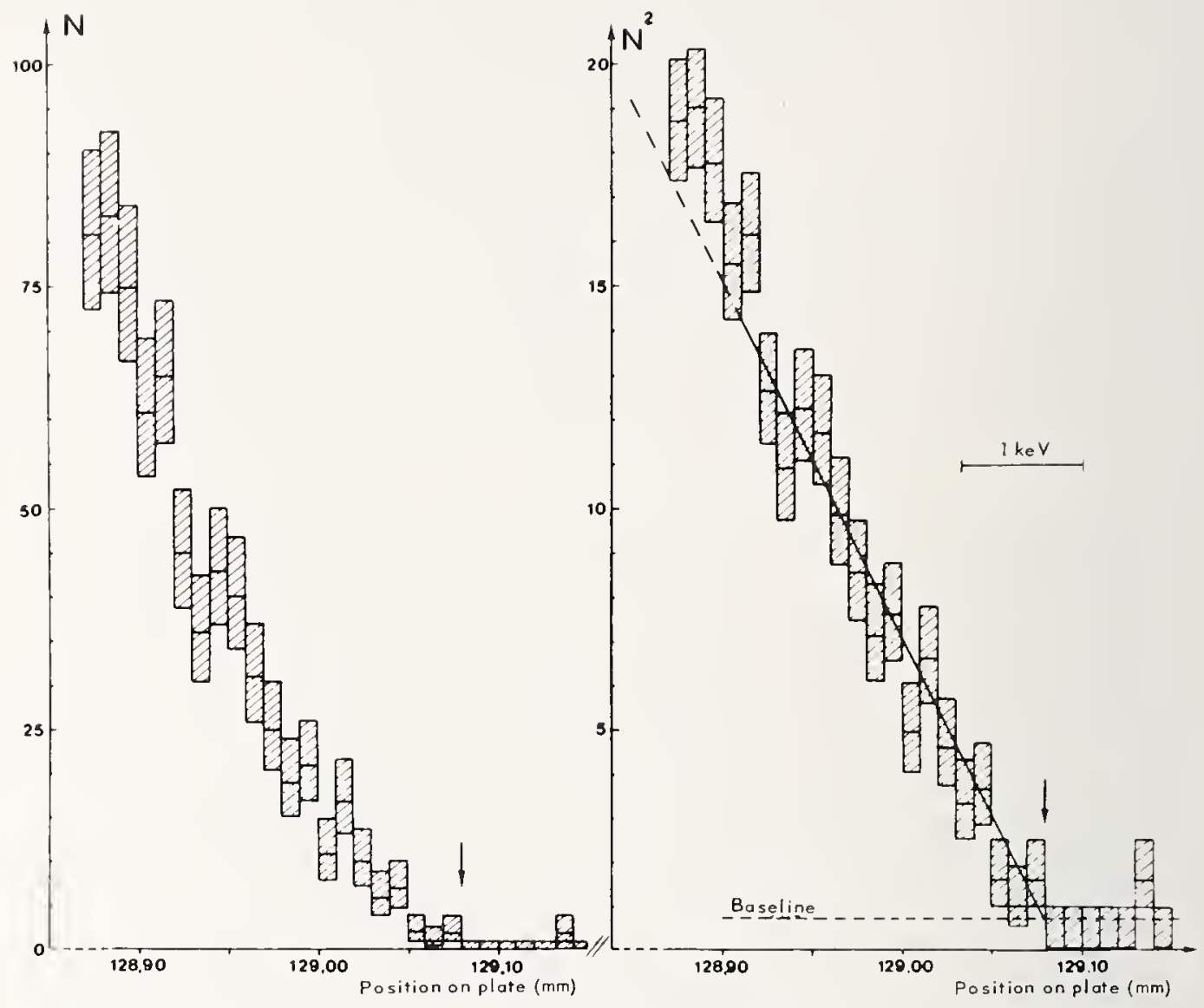

Figure 10. Edge of an $\alpha$-particle line; at the left the distribution of $N$, at the right that of $N^{2 / 3}$ : $\mathrm{N}$ : number of tracks counted (and statistical uncertainty) per band of $10 \mu \mathrm{m}$ width. The abscissa indicates the position on the photographic plate: the particle energy increases to the right. The extrapolated point, marked by an arrow, is the furthest point the particles reach. 


\section{Bibliography}

[1] Rytz, A., Standardization of ${ }^{40} \mathrm{Sr}^{+90} \mathrm{Y}$ by means of a chemical separation, in "Standardization of Radionuclides," IAEA. Vienna, pp. 247-256 (1967), and Recueil de Travaux du B.I.P.H. 1, (1966-1967).

[2] Rytz, A., Colas, C., and Veyradier, C., Some experiments on the dilution of radioactive solutions and the uniformity of mixing, Recueil de Travaux du B.I.P.M. 2, article 25, 8 pages (1968-1970).

[3] Colas, C. and Rytz, A., La résistance électrique des supports et des sources utilisés dans le comptage $4 \pi \beta-\gamma$, Recueil de Travàux du B.I.P.M. 3, article 18, 8 pages (19711972).

[4] Description of assembly No. 3 for counting by $4 \pi \beta(\mathrm{PC})-\gamma$ coincidences, Rapport BIPM$75 / 2$.

[5] See, for example, the appendices to CCEMRI, 4th session, 1963, and 5th session, 1964.

[6] CCEMRI, Section II, 1st meeting, p. R17 (1970).

[7] Müller. J. W.. Dead-time problems, Nucl. Instr. and Meth. 112, pp.47-57 (1973), and Recueil de Travaux du B.I.P.M. 4, article 20 (1973-1974).

[8] Müller. J. W., Some formulae for a dead-time-distorted Poisson process, Nucl. Instr. and Meth. 117, pp. $401-404$ (1974), and Recueil de Travaux du B.I.P.M. 4, article 21 (19731974).

[9] Müller, J. W.. Some notes on pair statistics, Recueil de Travaux du B.I.P.M. 4, article 24, 12 pages (1973-1974).

[10] Müller, J. W., A new method for distinguishing between pairs and single pulses, Recueil de Travaux du B.I.P.M.4, article 25, 10 pages (1973-1974).

[11] Müller, J. W.. A complex modulo K counter, Recueil de Travaux du B.I.P.M. 4, article 26, 16 pages (1973-1974).

[12] Grennberg, B. and Rytz, A., Absolute measurements of $\alpha$-ray energies, Metrologia 7, pp. 65-77 (1971), and Recueil de Travaux du B.I.P.M. 3, article 26 (1971-1972).

[13] Rytz, A., Catalogue of recommended alpha-energy and intensity values, Atomic Data and Nuclear Data Tables 12 , pp. $479-498$ (1973), and Recueil de Travaux du B.I.P.M. 4, article 31 (1973-1974). 



\section{CHAPTER X}

\section{AND GAMMA RAYS}

\section{Generalities}

\section{The Quantity "Exposure." The Principle of the Ionization Chamber With "Air Walls."}

Ionizing radiations are frequently used to produce modifications in matter-modifications sometimes physical (tests of materials for nuclear reactors), sometimes chemical (polymerization of plastics), sometimes biological (treatment of cancer). These radiations must be measured and checked at the time of use. In situations of this kind, the radiation is considered not from the point of view of the source producing it, but from that of the material which is irradiated: whence the name dosimetry (literally, measurement of dose) given to the measurement of quantities which characterize radiations of this kind.

As to $\mathrm{x}$ and $\gamma$ radiations, one uses a particular modification of matter which lends itself well to measurement: the ionization of air. Photon radiations are not directly ionizing: in the first step, the photons liberate fast electrons, ${ }^{1}$ these then progressively lose the energy which the photons have given them, by exciting and ionizing atoms in their path. When photons liberate electrons in a small volume $\mathrm{d} V$, the resulting ions appear in the entire neighboring region. This zone of influence has dimensions which vary with the energy of the radiation, from about a millimetre to several tens of centimetres in air, depending on whether it is a matter of hard or soft $x$ rays.

Whatever be their initial kinetic energy, the electrons practically always expend the same energy on the average in producing a pair of ions in a given medium. This energy, customarily designated by the symbol $\bar{W}$, is about $54 \times 10^{-19} \mathrm{~J}$ in air. The number of ions obtained is thus proportional to the energy transferred by the photons to the electrons and allows characterization of the beam of photons. This is why one defines at a point $\mathrm{M}$ of a

\footnotetext{
${ }^{1}$ In the field of interest to us, two processes are responsible for the production of electrons: the photoelectric effect in which the photon loses all its energy in expelling an electron from an atom, and the Compton effect, an elastic collision with a free electron in which the electron receives and carries away part of the energy of the incident photon, the remainder appearing in the form of a scattered photon.
} 


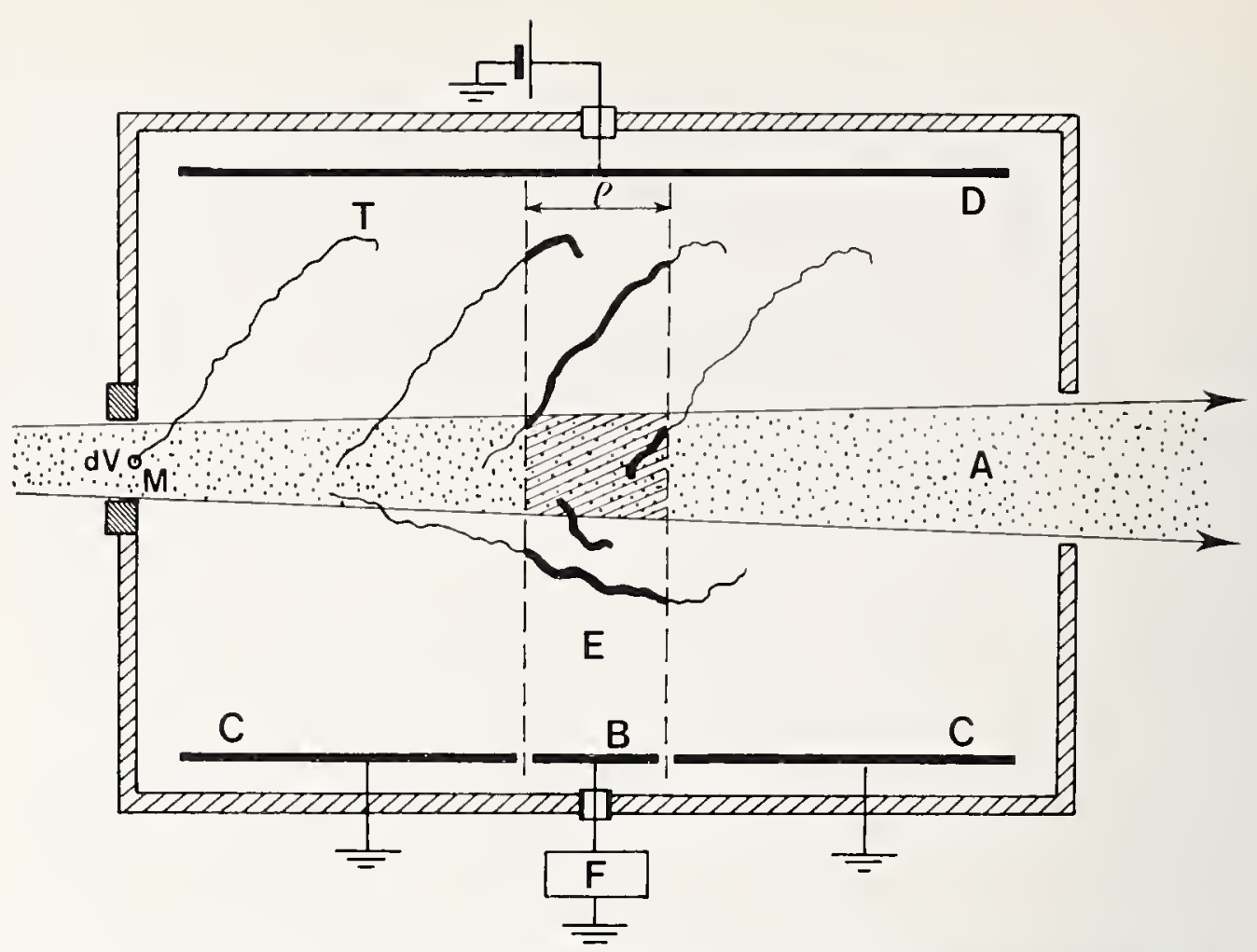

Figure 1. Principle of the air-wall ionization chamber. A: beam of $x$ rays: B: collector plate (potential $\sim 0$ ); $\mathrm{C}$ : grounded guard plate (potential 0); D: plate at a potential of a few kilovolts, the electric field of about $20000 \mathrm{~V} \cdot \mathrm{m}^{-1}$ thus created between D and B-C allows collection of the ions; $\mathrm{E}$ : region of length $l$ (several centimetres) from which are collected the ions reaching electrode $B$, the charge which they transport is measured by the device $F$. A few examples of electron paths are shown. only the ions produced on the portion of the paths shown in heavy lines are collected. The upper part of the figure illustrates a typical case of compensation; the three trajectory portions utilized are on the whole equivalent to the trajectory $T$.

beam of $\mathrm{x}$ or $\gamma$ radiation a quantity called exposure, ${ }^{2}$ derived from the electric charge carried by the ions produced in air by the electrons liberated by the photons in the mass of air $\mathrm{d} m$ contained in a small volume surrounding the point $M$.

The diagram of the air-wall ionization chamber shown in figure 1 indicates the principle of the measurement of exposure. Between two parallel plates, there is an electric field which can collect the ions produced by the pencil of $x$ rays passing through the center of the chamber. According to the above, determination of the exposure at the point $M$ in the diaphragm aperture requires measuring the total ionization produced by the electrons which come from the small volume $\mathrm{d} V$ surrounding $M$, and this ionization

2 The definition of exposure given by the International Commission on Radiation Units and Measurement (ICRU). Its latest formulation (1971) is as follows: "The exposure $X$ is the quotient of $\mathrm{d} Q$ by $\mathrm{d} m$, where $\mathrm{d} Q$ is the absolute value of the total charge of ions of one sign produced in air when all the electrons (negative and positive) liberated in a volume element of air of mass $\mathrm{d} m$ are completely stopped in air. The special unit of exposure is the röntgen $(\mathbf{R})$. $1 \mathrm{R}=2.58 \times 10^{-4} \mathrm{C} \cdot \mathrm{kg}^{-1}$." 
only. This is evidently impossible, for inside the chamber, the ions which interest us are inextricably mixed with those generated by electrons liberated along the path of the photon beam. But thanks to compensations like that indicated in figure 1 , the charge $Q$ collected from the central region of the chamber provides the desired information and consequently the exposure at M. ${ }^{3}$

This implies that the cross-hatched volume in the figure is surrounded on all sides by a sufficient thickness of air (hence the name "air-wall" chamber): in fact, if the diaphragm is too close to the hatched volume, the compensation will be only partial, also, if the two parallel plates are not sufficiently separated, some electrons will strike these plates and their paths will thus not be totally utilized for the production of ions in the air.

\section{The Concept of a Standard of Exposure}

It would seem natural to choose as a standard, the exposure at a designated point in a reference beam. But the definition of such a beam cannot be envisaged for $\mathrm{x}$ rays because an $\mathrm{x}$-ray tube emits complex radiation which is known only imperfectly. In the most favorable case of $\gamma$ radiation emitted by a radioactive source, stability of the beam implies that the geometry of the system (and also that of nearby objects which could be sources of scattered radiation) remains fixed.

By reason of these difficulties, the unit of exposure is not maintained by the use of a radiation beam. The role of standard is assigned to the instrument for measuring exposure: an ionization chamber can constitute a standard of exposure rate, ${ }^{4}$ since it permits determination of this quantity from the measurement of two base quantities: electric current and mass. ${ }^{5}$ But it is impossible to experimentally reproduce the ideal situation assumed in the definition of exposure: the measurement of this quantity can be made only indirectly, thanks, in particular, to the compensations indicated earlier. Whatever standard chamber is used, it is necessary to

\footnotetext{
${ }^{3}$ If the beam is parallel (and if we neglect the attenuation of photons associated with the liberation of electrons), the exposure at $M$ would be the quotient of $Q$ by the mass of air contained in the cross-hatched volume. In reality, the beam diverges, but (always if we neglect the attenuation of the photons) there are as many photons coming through the aperture as through successive sections of the beam perpendicular to the axis, only the area in which they are distributed being changed. Similarly, the production of electrons remains constant in the slabs of air successively traversed by the beam. From this it can be deduced that the exposure at $\mathrm{M}$ is equal, in the first approximation, to $Q / A l \rho$, where $A$ is the area of the aperture, $l$ the length of the collection region, and $\rho$ the mass density of the air (see [1] for a more complete analysis).
}

${ }^{4}$ Exposure rate $=$ exposure/time

${ }^{5}$ In fact, these two quantities are measured in an indirect way: this has been indicated above for the mass of ionized air $(=A l \rho)$, we shall see it later for the ionization current. 
determine various correction factors which allow, starting with experimental results, calculation of the value of exposure at a given point in the beam which conforms to the ideal conditions of the definition. Finally it is the uncertainty in the determination of these factors which limits the accuracy of measurements of exposure.

\section{Measurement of X Radiation}

\section{Measurements of Exposure at the BIPM}

Since the beginning of work in this field at the BIPM (1962), we have tried to obtain good measurement conditions, which presupposes a highly stable radiation emission. In an $\mathrm{x}$-ray tube, the $\mathrm{x}$ rays are produced by the impact of an electron beam on a tungsten target. The radiation emitted will be stable if the number and energy of the electrons striking the target are both constant, which implies simultaneous stabilization of the tube current and the applied high potential (5 to $150 \mathrm{kV}$ and 100 to $300 \mathrm{kV}$ for the BIPM tubes). This potential is supplied by an electrostatic generator; by adding a supplementary regulating device, we have reduced its already small fluctuations to a few parts in a hundred thousand. With respect to the current, instead of adjusting the filament heating directly, we have done it more closely by using an error signal from the fluctuations of tube current. In this way it is possible to achieve a stability in the neighborhood of a tenthousandth.

Figure 2 shows the $150-\mathrm{kV}$ x-ray tube and the BIPM air-wall ionization chambers. The quantity usually determined is the exposure rate. It is therefore necessary to measure the ionization current resulting from the collection of ions. This current is very small, of the order of a few picoamperes or a few tens of picoamperes. It is measured by the Townsend method: the ionization current charges a capacitor of capacitance $C$; the potential difference $\Delta U$, proportional to the time of irradiation $\Delta t$, appears across the terminals. The measurement consists of determining the time $\Delta t$ which corresponds to collection of the charge $C \Delta U$. With the aid of a device developed and constructed at the BIPM since 1963, we have achieved a precision of the order of a ten-thousandth in the measurement of these very small currents. This precision allows us to carry out studies of phenomena (see below) causing small variations of the ionization.

It has been stated much earlier that to obtain the exposure, various correction factors must be applied to the results of ionization measurements: it is necessary, for example, to take account of the attenuation of the photons by the air in the chamber, of the parasitic ionization due to scattered photons, etc. The BIPM has worked on improving the accuracy of the determination of these factors on which the measurement of exposure depends strongly. Thus the attenuation by air is measured by placing 


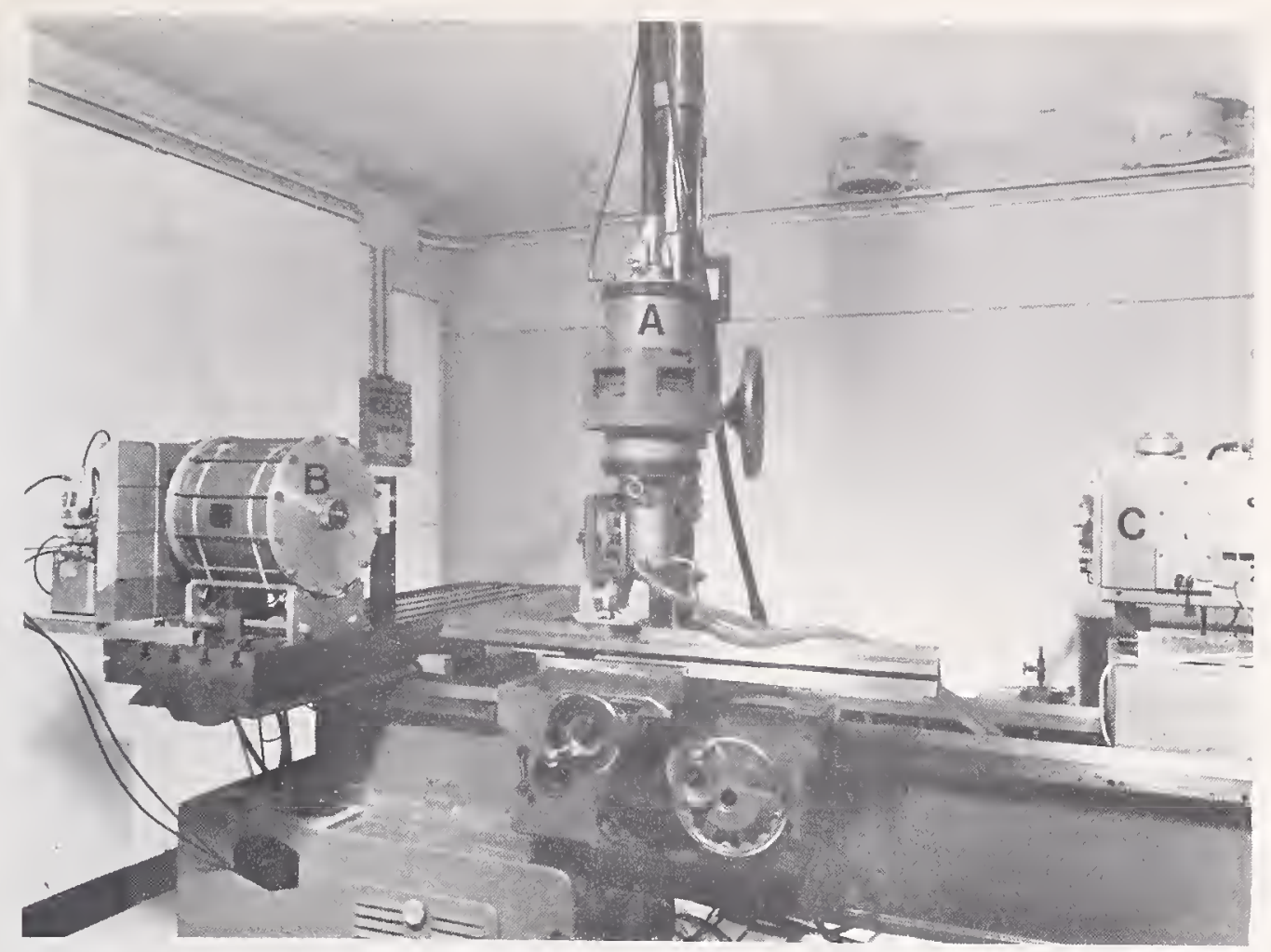

Figure 2. Measurement of exposure in a beam of $x$ rays. A: x-ray tube capable of being oriented so that either of the chambers B and C can be irradiated); B: standard chamber for soft x rays (plate spacing $7 \mathrm{~cm}$, collector potential $1500 \mathrm{~V}$ ); C: standard chamber for $\mathrm{x}$ rays of intermediate energy (plate spacing $18 \mathrm{~cm}$, collector potential $4000 \mathrm{~V}$ ).

an evacuated pipe between the tube and the chamber. Studies on two of these factors have been published. One of them [2] concerns the recombination of ions, a phenomenon which depends upon the applied electric field. Whatever field is used for the measurements, the collection of ions is incomplete (99.8 to $99.9 \%$ under usual conditions) and an extrapolation of the results to an infinite field is necessary. We have succeeded in determining experimentally the part which should be attributed to each of two types of recombination (initial recombination and volume recombination); this permits making a valid extrapolation for obtaining the saturation current.

Another experimental study has been devoted to the influence of water vapor on the ionization produced in an air-wall chamber [3]. An airwall chamber was placed in an airtight tank. A closed circuit connected to this tank comprised a pump producing gas circulation by compressing a flexible tube, a drying or humidifying device, and a dew-point hygrometer. Drying was obtained by use of a trap immersed in a mixture of methanol and dry ice; in this way no chemically foreign substance could pollute the air used in the chamber. If one takes account of the effect of water vapor on a) the liberation of electrons by photons, or b) the ionization produced by the electrons, and if one assumes that in these two cases the air and water 
vapor play entirely independent roles, a linear variation of ionization current as a function of water vapor content is predicted. The experimental results are very different from these predictions in the range of low humidity. Since the hypothesis of independent roles is very questionable as concerns b), the observed disagreement can be interpreted as due to a nonlinear variation of mean energy $\bar{W}$ needed to produce a pair of ions, as a function of the proportion of water vapor contained in the air (fig. 3).

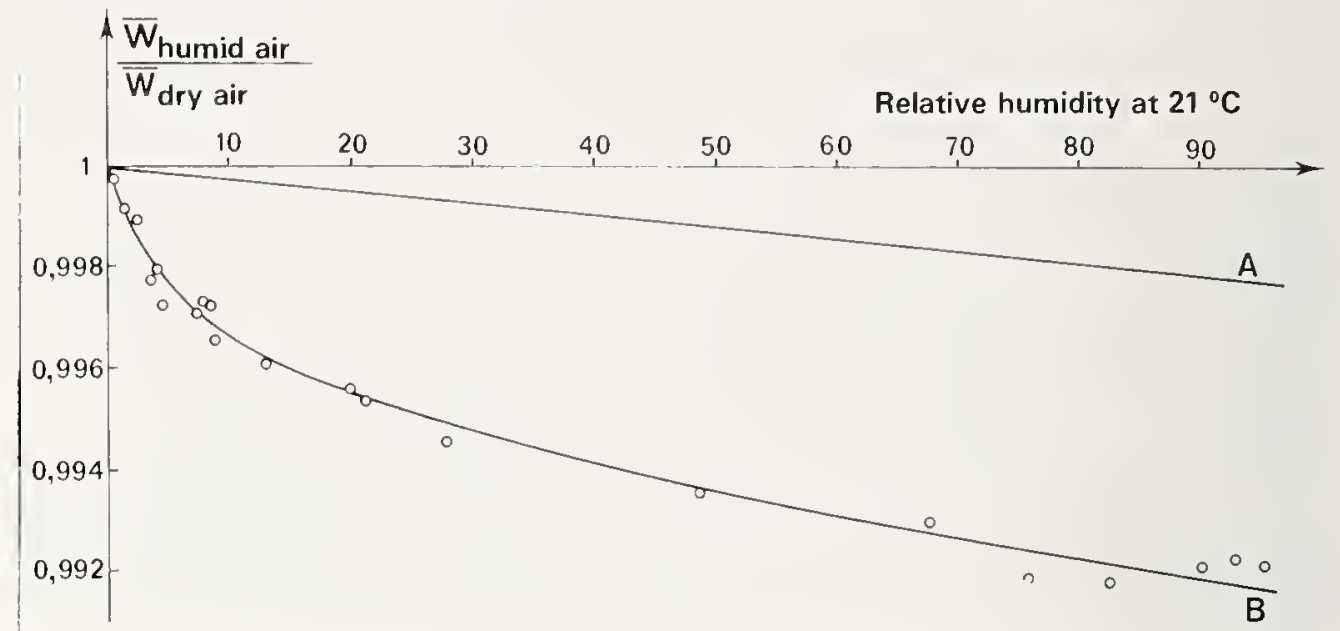

Figure 3. Variations of the mean energy $\bar{W}$ necessary for production of a pair of ions in air as a function of relative humidity.

Curve A corresponds to theoretical predictions, curve B is derived from experimental results.

\section{International Comparisons of Exposure Standards}

Direct international comparisons have been made at the BIPM since 1966 for several qualities of soft $x$ rays $(10-50 \mathrm{kV})$ between the standard chamber of the BIPM and some national exposure standards (United States of America, Canada, Netherlands, Sweden, Hungary) [4]. The differences $(0.5 \%$ at the most) are generally compatible with the estimated uncertainties. We have nevertheless observed systematic differences between standards of small dimensions (4-cm plate separation) and standards of larger dimensions (plate separations of 6 to $12 \mathrm{~cm}$ ) (fig. 4). This can be attributed to inaccurate corrections for the small standards, notably the correction for scattered photons (at $10 \mathrm{kV}$ ) and that for insufficient plate separation (at 50 $\mathrm{kV}){ }^{6}$ The tracking down of systematic errors is one of the interests in this type of comparison and the principal preoccupation of the BIPM.

\footnotetext{
${ }^{6}$ As we have seen earlier, if the plates are not sufficiently separated, we must make a correction since the trajectories of some electrons will not be fully used. This correction becomes more important when the radiation becomes more penetrating, for it then liberates more electrons of high energy, having long paths.
} 


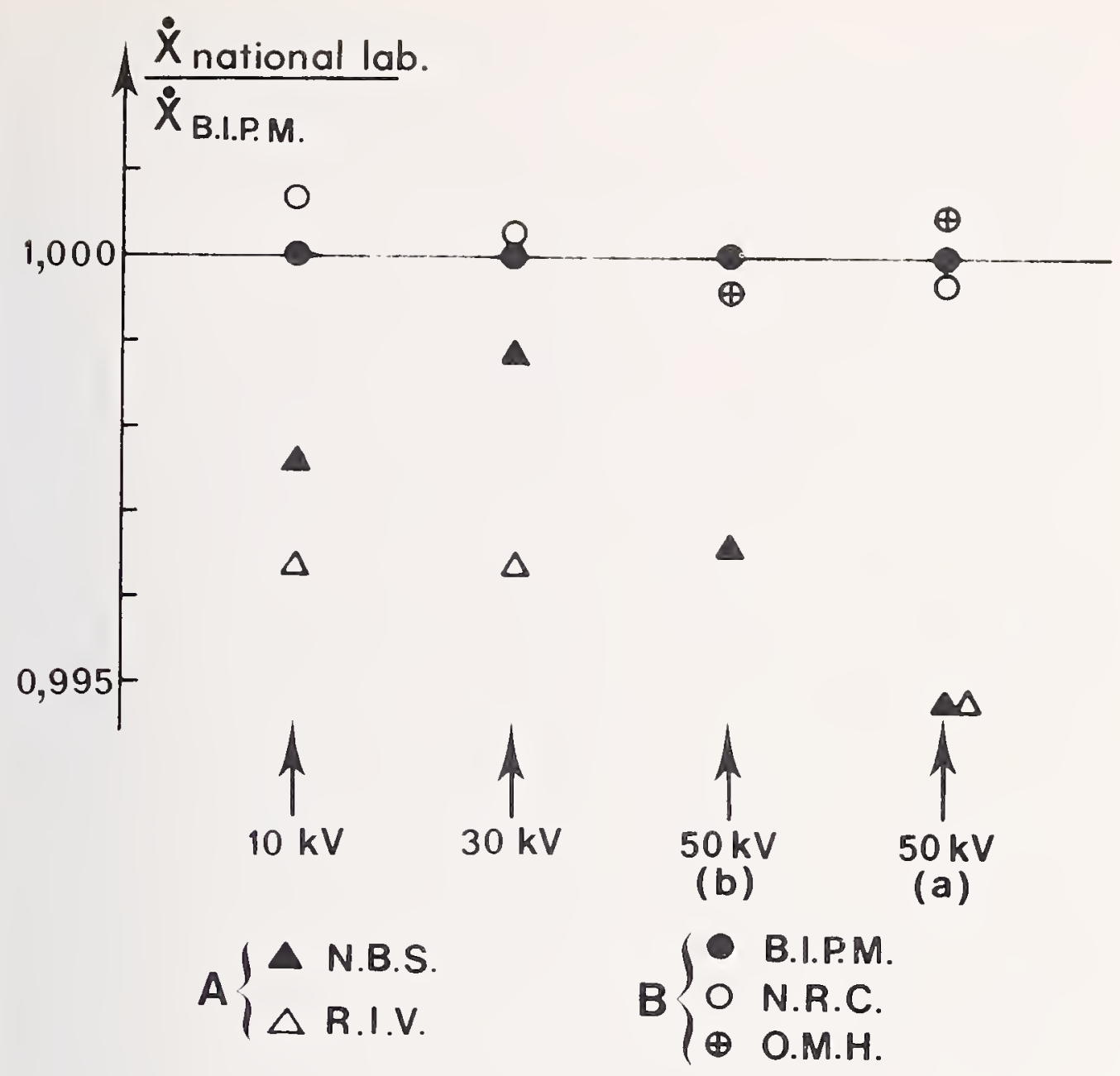

Figure 4. Some results of international comparisons in the field of low-energy $x$ rays. The figure gives the exposure rates $\dot{X}$ measured at the BIPM by the standards of different national laboratories, the BIPM standard being taken as reference. A: standards of small dimensions: B: stantarts of large dimensions. The figure shows a difference between the groups $\mathrm{A}$ and $\mathrm{B}$. This difference is accentuated when the radiation hecomes more penetrating (the $50 \mathrm{kV}$ radiation (a) is made more penetrating than the $50 \mathrm{kV}$ radiation (b) of the same energy by interposing in the path of the beam. a supplementary disk of aluminum, 3 mm thick, which stops the low-energy photons).

\section{Measurement of Gamma Radiation}

The $\gamma$ radiation from ${ }^{60} \mathrm{Co}$ plays a particularly important role in dosimetry, both because it is used very much in medicine and because it forms a sort of link between the low and medium energies $(<1 \mathrm{MeV})$ and the high energies ( 1 to $50 \mathrm{MeV}$ ). Measurements at high energy are frequently based on calibrations made with $\gamma$ radiation from ${ }^{60} \mathrm{Co}$. For this reason, the BIPM has chosen to give priority to measurements of this radiation. 


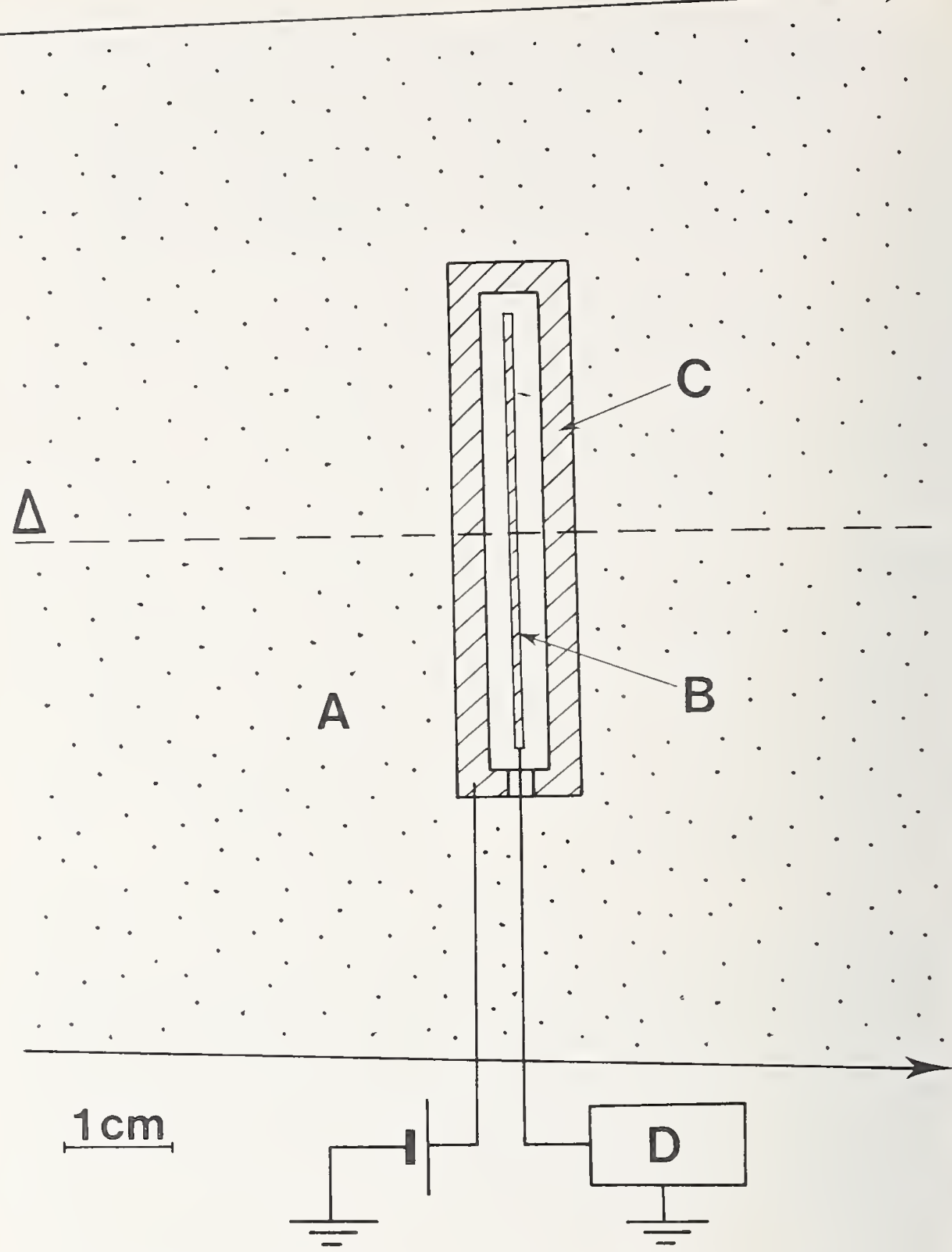

Figure 5. Schematic section of the cavity chamber with graphite walls, at the BIPM. A: heam of $\gamma$ rays irradiating the entire chamber - this radiation liberates. in the graphite. electrons which ionize the air in the cavity; B: graphite collecting electrode (potential $\approx 0$ ): $\mathrm{C}$ : graphite walls at a potential of $80 \mathrm{~V}$; the electric field $\left(\approx 40000 \mathrm{~V} \cdot \mathrm{m}^{-1}\right)$ thus created between $B$ and $C$ allows collection of the ions produced in the cavity. The ions of one sign ( + in the case of the figure) terminate on electrode B: the charge they carry is measured by the device $D$. The chamher is cylindrical; its circular faces are perpendicular to the axis $\Delta$ of the beam: the cavity volume is about $6.8 \mathrm{~cm}^{3}$. 
A source of ${ }^{60} \mathrm{Co}$ emits photons of 1.17 and $1.33 \mathrm{MeV}$. The paths of electrons liberated by these photons can attain several metres in at. mospheric air. In this case, exposure measurement with an air-wall chamber at atmospheric pressure poses many problems. One can try to avoid them by increasing the pressure to reduce the chamber dimensions, but then other difficulties appear (loss by recombination of ions can reach 10 percent if the pressure is of the order of 10 atmospheres). A different method would consist of utilizing a magnetic field created by a coil having the same axis as the photon beam: with a flux density of $0.25 \mathrm{~T}$, the electrons can be kept in a cylinder of approximately 10-cm diameter [5]. Unfortunately, this scheme permits the reduction of only the transverse dimensions (perpendicular to the beam axis) of the air-wall chamber. No solution using an air-wall chamber proves to be satisfactory. That is why the BIPM has chosen for its exposure standard a cavity chamber with graphite walls (fig. 5) in which several millimetres of graphite replaces several metres of air and is sufficient to assure "electron equilibrium."

This substitution of graphite for air brings a modification of the electron production which must first be considered. Moreover, one no longer operates in a simple medium: it is by virtue of the energy dissipated in one medium (air) by the electrons liberated in another medium (graphite) that there are ions to be measured. This results in a correction (called stopping power ${ }^{7}$ ) because the dissipation of energy of electrons in air is different from what it would be in graphite. The lack of accuracy (about $0.5 \%$ ) of this correction constitutes the principal defect of cavity chambers.

\section{The Installation at the BIPM, and the Exposure Measurements}

Although several millimetres of lead suffices for protection against $\mathbf{x}$ rays of intermediate energy, a thickness of some 20 centimetres is needed when it is a question of the radiation emitted by a ${ }^{60} \mathrm{Co}$ source having an activity of several hundred curies such as those used for exposure measurements. ${ }^{8}$ The BIPM therefore started by studying and having constructed

\footnotetext{
${ }^{7}$ For electrons of a given energy, the quotient $\mathrm{d} E / \mathrm{d} l$ is called the stopping power $S$ of a substance, where $\mathrm{d} E$ is the energy lost by an electron of energy $E$ in traversing an element of path of length $\mathrm{d} l$ in the substance considered. The electric charge due to ions produced in the air

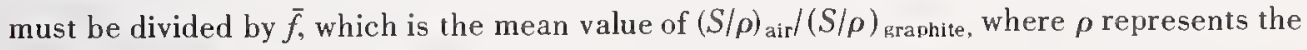
mass density of the corresponding substance.

${ }^{8}$ At $1 \mathrm{~m}$ from a source of $500 \mathrm{Ci}$, the exposure rate is about $10 \mathrm{R} / \mathrm{min}$ (which corresponds approximately to an ionization current of $250 \mathrm{pA}$ for a $5-\mathrm{cm}^{3}$ cavity chamber). After penetrating $25 \mathrm{~cm}$ of lead, this exposure rate is reduced to less than $2 \mathrm{mR} / \mathrm{h}$, which is less than the official permissable dose.
} 
(1965) a lead "castle" capable of holding up to six radioactive sources. Figure 6 shows schematically the essentials of this device which was designed to reduce to a minimum the radiation scattered into the beam defined by the collimator. This castle received two sources of $1 \mathrm{Ci}$ in 1966 and one of $480 \mathrm{Ci}$ (specific activity $400 \mathrm{Ci} / \mathrm{g}$ ) in $1968 .{ }^{9}$ This last source is currently used for exposure measurements.

The determination of exposure by use of a cavity chamber raises a certain number of problems. Some have been mentioned above, but those are not the only ones: in particular, if one proposes to characterize the $\gamma$-ray beam by means of the exposure which would exist in air in the absence of the chamber, it is necessary to know quantitatively the perturbation that the presence of the measuring instrument has on the quantity which one wishes to measure. This effect is usually studied by increasing the watl thickness of the chamber (above the $3 \mathrm{~mm}$ which is necessary to assure electron equilibrium) and extrapolating to zero thickness the variation observed in the ionization current as a function of wall thickness. By this procedure one measures in fact the superposed effects of several phenomena produced in the walls (photon attenuation and production of scattered photons of which some are in turn absorbed and contribute to the measured ionization) and the extrapolation is applied to the variation of a

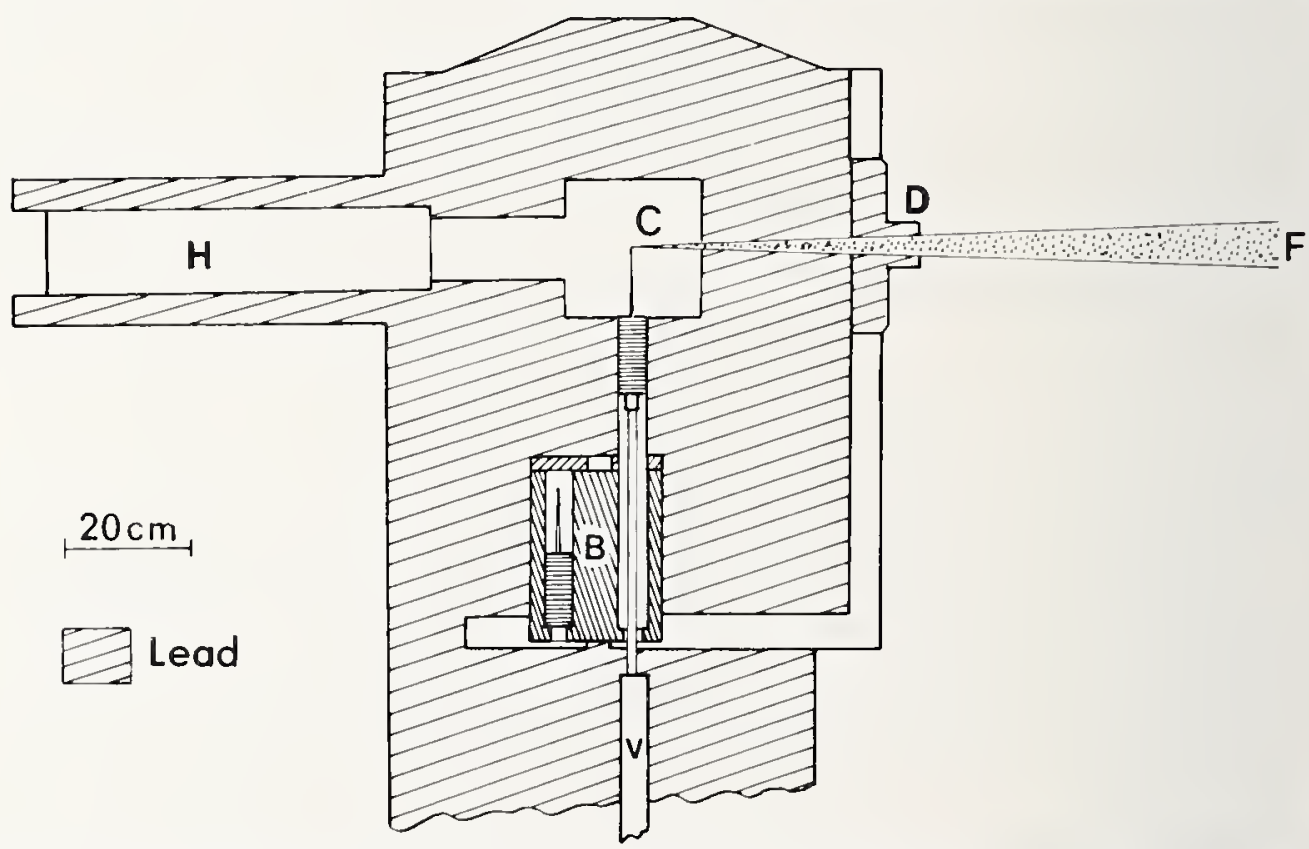

Figure 6. Schematic section of the lead castle for intense radioactive sources. Any of the sources contained in the barrel $B$ can bc chosen, then raiscd with the aid of an electric jack $V$ to the source chamber $C$. The port $H$ (lead glass) allows observation that the chosen source is correctly centered on the axis of the collimator D which limits the useful beam $\mathrm{F}$. (To simplify the figure, many details, for example the device for rotating the barrel $B$, have been omitted.)

\footnotetext{
${ }^{9}$ These sources were given to the BIPM, those of $1 \mathrm{Ci}$ by the National Research Council (Canada), that of 480 Ci by the National Bureau of Standards (U.S.A.).
} 
sum whose different terms do not vary in the same manner with thickness. This is why we have tried to study these phenomena separately at the BIPM. For example, if we place a graphite disk either on the source side of the collimator aperture, or next to the chamber, the attenuation of photons stays the same but the ionization current is a little larger in the second case, the difference corresponding to the contribution from scattered photons. ${ }^{10}$ In a general way, the BIPM has attacked the problem of the cavity chamber from a new angle by trying to approach as closely as possible the conditions of the theoretical analysis [1]. For this reason, we have chosen a flat cylindrical cavity (fig. 5). Among the problems to be resolved, one of the most difficult is the following: how to take account of the nonuniformity of the radiation field in which are placed the chamber walls which produce the electrons, and in particular the nonuniformity of this field parallel to the beam axis, which becomes important if the cavity size is increased or if the chamber is closer to the source. This last problem has been studied theoretically [6] and experimentally by varying the sourcechamber distance. Unfortunately, the role of the side walls of the chamber can not be analyzed validly in a simple way ${ }^{11}$ and this probably increases the uncertainty of the determination of exposure by 0.3 percent. As for the reproducibility of measurement, it is excellent since the long-term standard deviation (for the most intense source), calculated for results extending over a year and a half, is equal to $10^{-4}$, allowing for weakening of the source whose half-life is 1926 days.

\section{International Comparisons of Exposure Standards for the ${ }^{60} \mathrm{Co} \gamma$ Radiation}

International comparisons have taken place at the BIPM between the standard chamber of the BIPM and some national standards of exposure (United States of America, Federal Republic of Germany, Netherlands, Hungary). These standards are also graphite cavity chambers but these chambers, with one exception, have forms and dimensions differing from that of the BIPM. Their total wall corrections are determined by the method indicated earlier. Figure 7 shows a satisfactory agreement, allow ing for the uncertainties associated with different chambers.

There do not appear to be any systematic differences due to the chamber geometries or to the methods of correcting for wall effects. Unfortunately, this type of comparison does not permit uncovering systematic errors involving the ratio $f$ of the stopping powers of air and graphite. These

\footnotetext{
${ }^{10}$ For a disk of $3.3 \cdot \mathrm{mm}$ thickness, the attenuation produces a loss of 3.6 percent and the scattered photons a gain of 2.0 percent.

11 The contribution of the side walls represents 14 percent of the total ionization measured. It was determined experimentally with a chamber similar to the standard chamber, but having side walls as thin as possible $(0.5 \mathrm{~mm})$. We added rings successively until electron equilibrium was reached and extrapolated the observed variation to zero thickness.
} 
errors are practically the same for all graphite chambers, and therefore are eliminated when one determines the ratio of exposure measured by two of them.

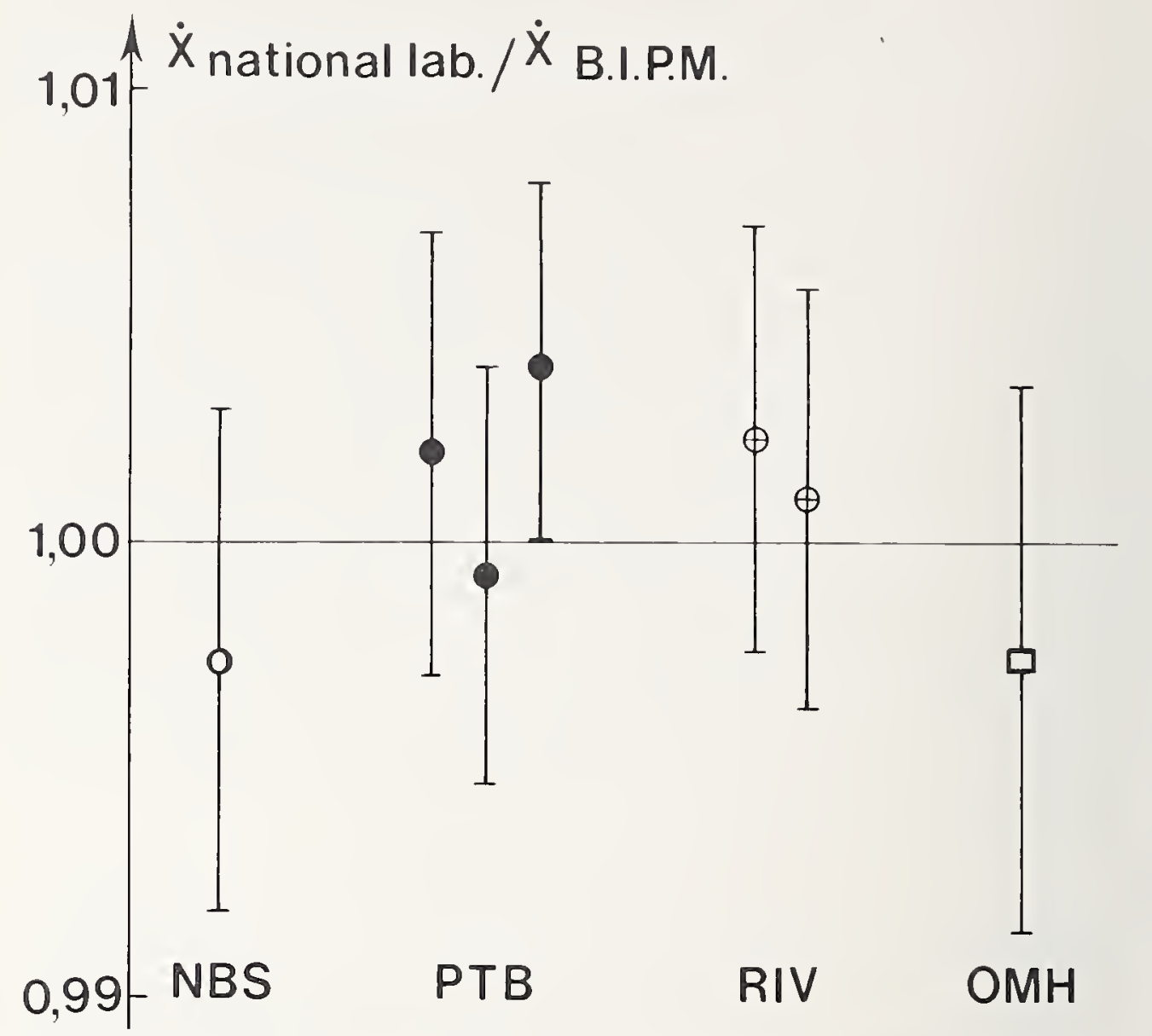

Figure 7. International comparison of exposure standards for the radiation from ${ }^{60}$ Co. The figure gives the exposure rates $\dot{X}$ measured at the BIPM by the standards of different national laboratories, the BIPM standard being taken as reference. The error bars represent the estimates of systematic uncertainty (the random uncertainties are negligible).

\section{Comparison of Activity and Exposure Measurements}

The systematic errors which have just been noted can, on the contrary, be brought out when one compares, for a given cobalt source, measurements of two quantities of different kinds such as activity and exposure. Two photons (1.17 and $1.33 \mathrm{MeV}$ ) are emitted at each disintegration of an atom of ${ }^{60} \mathrm{Co}$. The activity is the number of disintegrations per unit time; it thus permits knowledge of the incident energy of the photons crossing unit area per unit time at a given distance from the source. A known fraction ${ }^{12}$ of this energy is transferred to the electrons liberated into the medium,

12 This fraction is the coefficient of energy transfer, which is easily calculated from the probabilities of photoelectric and Compton interactions. 
which we assume to be air. Knowing that a mean energy $\bar{W}$ is needed to produce a pair of ions in air, the corresponding ionization, and thus the exposure, is easily deduced.

For this activity-exposure comparison, we use one of the two 1-curie sources (say $3.7 \times 10^{10} \mathrm{~s}^{-1}$ ). Although it corresponds to a small exposure rate, this activity is much too high to be measured directly. Measurement of the activity $A_{\mathrm{S}}$ of this source $\mathrm{S}$ was carried out by an original method utilizing a second source $\mathrm{G}$ of the same geometry, but having a much lower activity, $A_{\mathrm{G}} \approx 2 \mathrm{mCi}$ [7], as an intermediary. This method involves two steps: a) a relative measurement, the ratio $R=A_{\mathrm{S}} / A_{\mathrm{G}}$ of the activities of the two sources; and b) an absolute measurement of the activity $A_{\mathrm{G}}$. Measurement a) is made with a scintillation counter system. ${ }^{13}$ The sources $\mathrm{G}$ and $\mathrm{S}$ are placed at distances $D_{\mathrm{G}} \approx 1 \mathrm{~m}$ and $D_{\mathrm{S}} \approx 23 \mathrm{~m}$ from the diaphragm limiting the useful beam so that the number of photons incident per unit time at the diaphragm is practically the same for the two sources; this eliminates many of the systematic errors. ${ }^{14}$ The ratio $R$ is given, to within a few corrections, by the ratio of the squares of the distances, $D_{\mathrm{s}}{ }^{2} / D_{\mathrm{G}}{ }^{2}$, multiplied by the ratio of the pulse count rates, $N_{\mathrm{S}} / N_{\mathrm{G}}$. Measurement b) made by the $4 \pi \beta$ $\gamma$ coincidence counting method, consists in determining the activities $A_{m}$ of a series of $m$ very weak sources (about $0.2 \mu \mathrm{Ci}$ ) obtained in the following way: the source $G$ is dissolved in nitric acid and the solution is diluted in a known ratio, the sources $m$ comprise small quantities of this diluted solution, each containing a fraction $1 / F\left(\approx 10^{-4}\right)$ of the source $G . A_{\mathrm{G}}$ is then the mean value of the products $F A_{m}$, and $A_{\mathrm{S}}$ is the product $R A_{\mathrm{G}}$; this value $A_{\mathrm{S}}$ which one finally reaches is known with a good accuracy (better than $0.2 \%$ ) despite all the intermediate steps brought into play. ${ }^{15}$

In comparing the measured exposure rate at a given distance from the source $\mathrm{S}$ to the calculated exposure rate based on the value $A_{\mathrm{S}}$, a difference of 0.8 percent is found. This difference can arise either from the inaccuracy of the exposure measurement (due mainly to the ratio $f$ of the stopping powers of air and graphite), or from the value of $\bar{W}$ (mean energy needed to produce a pair of ions) which had been used in the calculation of exposure rate from activity. It is therefore necessary to carry on the efforts to improve the accuracy of such constants as $f$ and $\bar{W}$.

\footnotetext{
${ }_{13}$ The detector is a crystal of sodium iodide in which part of the energy dissipated by the electrons liberated by the $\gamma$-ray photons appears in the form of luminous energy: one observes flashes in the crystal, called scintillations, which are transformed into pulses of electric volt age by means of a photomultiplier. An electronic device allows counting these pulses, whose total number is proportional to that of the $\gamma$-ray photons striking the crystal.

${ }^{14}$ Note that without other precautions, the number of photons would be reduced by about 14 percent in the case of the source $S$, by the additional 22 metres of air. To avoid this important correction, the beam passes down an evacuated pipe.

15 The validity of this result is confirmed by the excellent agreement $(0.02 \%)$ hetween the two values of the activity of a single source, one obtained by this method, the other by determining the power emitted by the source (calorimetric measurement at the National Bureau of Standards) [8].
} 


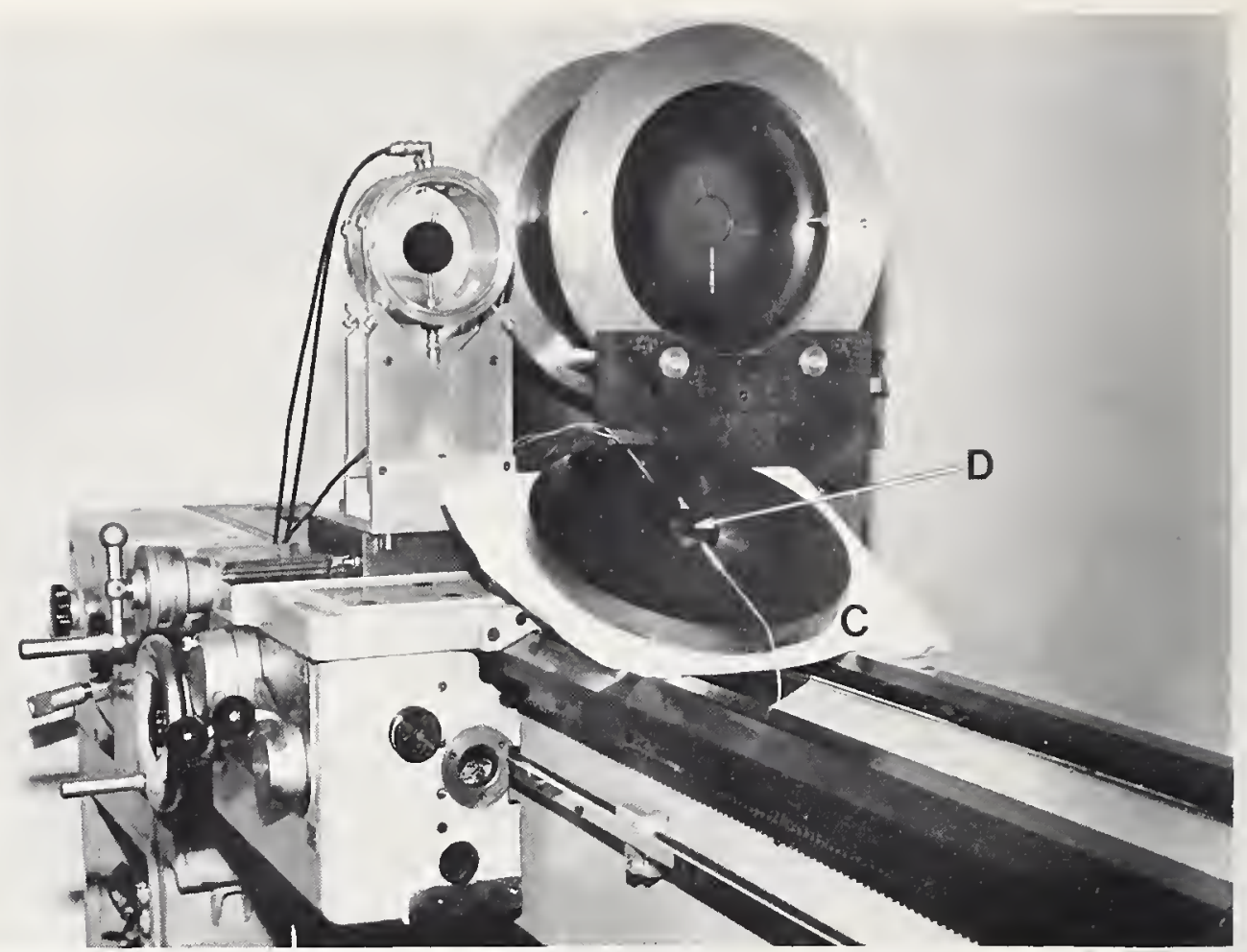

Figure 8. Measurement of exposure and absorbed dose in graphite, for $\gamma$ radiation from ${ }^{60} \mathrm{Co}$. A: standard cavity chamber for exposure measurements: B: graphite phantom whose front cover is shown in the foreground with the cavity chamber D used for measurement of $a b$ sorbed dose.

\section{Absorbed Dose}

In 1973, the BIPM commenced measurements of another quantity which is fundamental in dosimetry: absorbed dose, the energy dissipated per unit mass in a volume element of the material under consideration. The CCEMRI has chosen graphite as the reference material, the standard of absorbed dose being implicitly represented by a graphite cylinder of given dimensions (the name "phantom" assigned to this cylinder originates from medical reasons, for it simulates a human body with respect to the energy given it by radiation).

The BIPM uses the ionization method (fig. 8), as for the measurement of exposure. If the energy $\bar{W}$ is known, the ionization produced permits calculation of the dose absorbed in the air of the cavity and from this is derived the dose absorbed in the graphite with the help of the ratio of stopping powers of graphite and air. The determination of this ratio is more complex in this case since the electrons are no longer liberated by only 1.17 and $1.33 \mathrm{MeV}$ photons: in fact, the deeper the beam penetrates into the phantom, the more it is enriched in scattered photons in proportion to its impoverishment in photons of 1.17 and $1.33 \mathrm{MeV}$. The photon spectrum has been calculated for the BIPM phantom by the Monte Carlo method, 
simulating 25000 photon "histories" with the aid of the IBM 1130 calculator at the BIPM. Another problem to be resolved is that of the thickness of the chamber, whose cavity can not be likened to an elementary volume. It will be very fruitful to compare in the near future, the ionometric absorbeddose standard of the BIPM with the standards of national laboratories, in particular with those which use the calorimetric method for measuring the energy imparted by the radiation.

\section{Bibliography}

[1] Allisy, A., Contribution à la mesure de l'exposition produite par les photons émis par le ${ }^{60} \mathrm{Co}$, Metrologia 3,41-51 (1967), and Recueil de Travaux du B.I.P.M. 1, (1966-1967).

[2] Niatel, M.-T., An experimental study of ion recombination in parallel-plate free-air ionization chambers, Phys. Med. Biol. 12 , pp. 555-563 (1967), and Recueil de Travaux du B.I.P.M. 1, (1966-1967).

[3] Niatel, M.-T., Etude expérimentale de l'influence de la vapeur d'eau sur l'ionisation produite dans l'air, C.R. Acad.Sci. Paris 268-B, pp. 1650-1653 (1969), and Recueil de Travaux du B.I.P.M. 2, article 22 (1968-1970).

[4] Boutillon, M., Henry, W. H., and Lamperti, P. J., Comparison of exposure standards in the 10-50 kV x-ray region, Metrologia 5, pp. 1-11 (1969), and Recueil de Travaux du B.I.P.M. 2, article 21 (1968-1970).

[5] Allisy, A. and Boutillon, M., Sur l'utilisation d'un champ d'induction magnétique dans les mesures d'exposition de rayons X et $\gamma, C . R$. Acad. Sci. Paris 260, pp. 6576-6579 (1965).

[6] Boutillon, M., and Niatel, M.-T., A study of a graphite cavity chamber for absolute exposure measurements of ${ }^{60} \mathrm{Co}$ gamma rays, Metrologia 9, pp. 139-146 (1973), and Recueil de Travaux du B.I.P.M. 4, article 16 (1973-1974).

[7] Roux, A.-M., A method of measuring the activity of a $1 \mathrm{Ci}^{60} \mathrm{Co}$ source. Metrologia $\mathbf{1 0}$, 99-102 (1974), and Recueil de Travaux du B.I.P.M. 4, article 17 (1973-1974).

[8] Roux, A.-M., Comparison of activity and power measurements of a $1 \mathrm{Ci}{ }^{61} \mathrm{Co}$ source, Metrologia 10, 103-104 (1974), and Recueil de Travaux du B.I.P.M. 4., article 18 (19731974). 



\section{CHAPTER XI}

\section{NEUTRON MEASUREMENTS}

\section{Gemeralities}

Neutrons and protons, which are the constituents of atomic nuclei and thus of matter, resemble each other strongly in some respects. Neutrons, however, have no electric charge and thus they can pass without significant perturbation through the cloud of electrons which surrounds the nuclei of atoms. They can therefore easily penetrate matter without ionizing the atoms, and reach to the nuclei, causing nuclear reactions. These properties play an essential role in the production, detection, and utilization of neutrons. In particular, certain nuclear reactions which they produce release a very large amount of energy. It is especially the utilization of this energy on a large scale which brought about the urgent need for exact neutron measurements.

\section{Neutron Sources}

By bombarding light elements such as beryllium, boron, and lithium with helium nuclei ( $\alpha$ particles), one can produce neutrons ( $n$ ) by reactions denoted $(\alpha, n)$, for example:

$$
{ }_{4}^{9} \mathrm{Be}+{ }_{2}^{4} \mathrm{He} \rightarrow{ }_{6}^{12} \mathrm{C}+{ }_{0}^{1} \mathrm{n},
$$

or according to the usual notation, ${ }^{19} \mathrm{Be}(\alpha, \mathrm{n})^{12} \mathrm{C}$. Let us note in passing that it was this reaction which led J. Chadwick, in 1932, to the historic discovery of the neutron. Thus a mixture of beryllium and an $\alpha$-particle emitter, such as radium or americium, provides a convenient $(\alpha, n)$ neutron source.

Most nuclei are capable of emitting a neutron when struck by a sufficiently energetic $\gamma$ photon. For this $(\gamma, n)$ reaction to take place, the energy of the photon must be equal to or greater than the binding energy of the

\footnotetext{
${ }^{1}$ This notation indicates, in order, the target nucleus $\left({ }^{9} \mathrm{Be}\right)$. the incident particle $(\alpha)$, the ejected particle $(\mathrm{n})$, and the final nucleus $\left({ }^{12} \mathrm{C}\right)$. The subscript, often omitted, indicates the number of protons (atcmic number); the superscript is the sum of the numbers of protons and neutrons (mass number).
} 
neutron; this energy is generally between 6 and $8 \mathrm{MeV}$, except for beryllium (1.6 MeV) and deuterium (2.2 MeV):

$$
\begin{gathered}
{ }_{4}^{9} \mathrm{Be}+\gamma \rightarrow{ }_{4}^{8} \mathrm{Be}+{ }_{0}^{1} \mathrm{n} \rightarrow 2{ }_{2}^{4} \mathrm{He}+{ }_{0}^{1} \mathrm{n} \\
{ }_{1}^{2} \mathrm{H}+\gamma \rightarrow{ }_{1}^{1} \mathrm{H}+{ }_{0}^{1} \mathrm{n} .
\end{gathered}
$$

Thus a capsule of radium surrounded by beryllium constitutes a currently used $(\gamma, n)$ source, the radium emitting a large number of $\gamma$-rays of energy above 1.6 MeV. However the most used and most intense neutron sources at present are nuclear reactors.

Neutrons produced by $(\alpha, n)$ and $(\gamma, n)$ sources or by nuclear reactors generally have a broad energy spectrum. In order to produce a beam of fast monoenergetic neutrons, charged particle accelerators are needed. Some of the reactions used with charged particle accelerators are:

$$
\begin{gathered}
{ }^{2} \mathrm{H}(\mathrm{d}, \mathrm{n}){ }^{3} \mathrm{He},{ }^{3} \mathrm{H}(\mathrm{p}, \mathrm{n}){ }^{3} \mathrm{He},{ }^{3} \mathrm{H}(\mathrm{d}, \mathrm{n}){ }^{4} \mathrm{He}, \\
{ }^{7} \mathrm{Li}(\mathrm{p}, \mathrm{n}){ }^{7} \mathrm{Be},{ }^{12} \mathrm{C}(\mathrm{d}, \mathrm{n}){ }^{13} \mathrm{~N},{ }^{11} \mathrm{~B}(\alpha, n){ }^{14} \mathrm{~N}, \text { etc. }{ }^{2}
\end{gathered}
$$

\section{Detection of Neutrons}

Since the neutron does not ionize the atoms it encounters, the usual methods of detection are not applicable and one must have recourse to other effects. The interaction of free neutrons with the matter they traverse is shown by their scattering and absorption. The scattering (elastic and inelastic collisions) slows the neutrons which transfer energy to the nuclei they encounter; the nuclei can acquire, by recoil, enough energy to produce ions. The absorption of neutrons causes nuclear transformations. Essentially three types of detection are seen: a) by collision, particularly by elastic collision of fast neutrons with protons; the kinetic energy of the neutrons is partially converted to recoil energy of protons which can then be measured through ionization produced or by light emitted; b) by counting light particles $(\mathrm{p}, \mathrm{d}, \alpha, \ldots)$ produced in the course of a nuclear reaction; c) by analysis of resulting products (chemical analysis, analysis by the radioactivity produced, etc.).

\section{Neutron Measurements}

When a beam of neutrons is incident on a target (a body containing a large number of atoms of a certain kind), only a small fraction of the incident neutrons passes sufficiently close to target atoms for the reaction under study to take place. The beam is characterized by its flux density $\Phi$,

${ }^{2} \mathrm{p}={ }^{\mathrm{i}} \mathrm{H}=$ proton (hydrogen nucleus); $\mathrm{d}={ }^{2} \mathrm{H}=$ deuteron (deuterium nucleus); ${ }^{3} \mathrm{H}=$ triton (tritium nucleus); $\alpha={ }^{4} \mathrm{He}$ (nucleus of helium -4 ); ${ }^{3} \mathrm{He}=$ (nucleus of helium-3). 
the number of neutrons crossing a unit area per unit time. If the target contains a total number $N$ of nuclei and if each of these nuclei presents an area $\sigma$ to the beam, the mean number of times per second that a nucleus interacts with a neutron is equal to the product $N \Phi \sigma$ and is called the reaction rate. The quantity $\sigma$, called the cross section for this reaction, must not be confused with the (very small) geometric cross section of the nuclei or of the neutron; it is a fictitious area which in fact measures the probability of occurrence of the reaction under consideration.

The measurement of cross sections holds a very important position in the field of neutron measurements and requires the determination of neutron flux density (there is however one exception: the cross section multiplied by the number of nuclei per unit volume in an absorber gives the attenuation coefficient; this latter can be determined by measurements of transmission through a sheet of material, which requires only the measurement of a ratio of flux densities).

Furthermore, precise measurements of flux density are also necessary for standardizing detectors used in various fields (nuclear physics, neutron dosimetry, protection against ionizing radiation, industry, etc.). This quantity has been judged sufficiently important for the BIPM to undertake a program in this area. The BIPM has for this purpose two types of neutron sources which will be described in the following sections, along with the methods used to measure neutron flux density.

\section{Measurement of the Emission Rate of a Neutron Source by the Manganous Sulfate Bath Method}

Here we are concerned with neutron sources of the types $(\alpha, n)$ or $(\gamma, n)$ which use long half-life radioactive emitters such as radium ( 1600 years) or americium (433 years). The emission rate of such a source is defined by the number of neutrons emitted per second (into all directions). Figure 1 gives the dimensions and compositions of four neutron sources of this kind possessed by the BIPM.

Measurements of emission rate of these neutron sources are carried out by a classical method of total absorption of the neutrons in a concentrated aqueous solution of manganous sulfate. The fast neutrons emitted by the source are first slowed down, primarily by scattering by hydrogen; they then have a high probability of being captured when they are in thermal equilibrium with the medium (thermal neutrons). The dimensions of the spheres which contain the manganous sulfate solution are chosen according to the energy spectrum of the neutrons emitted by the source to be measured, to be large enough to reduce the loss of neutrons from the sphere to an acceptable value; for $(\alpha, n)$ sources whose spectrum extends up to a maximum energy of $12 \mathrm{MeV}$, the sphere has a diameter of $1 \mathrm{~m}$ and 


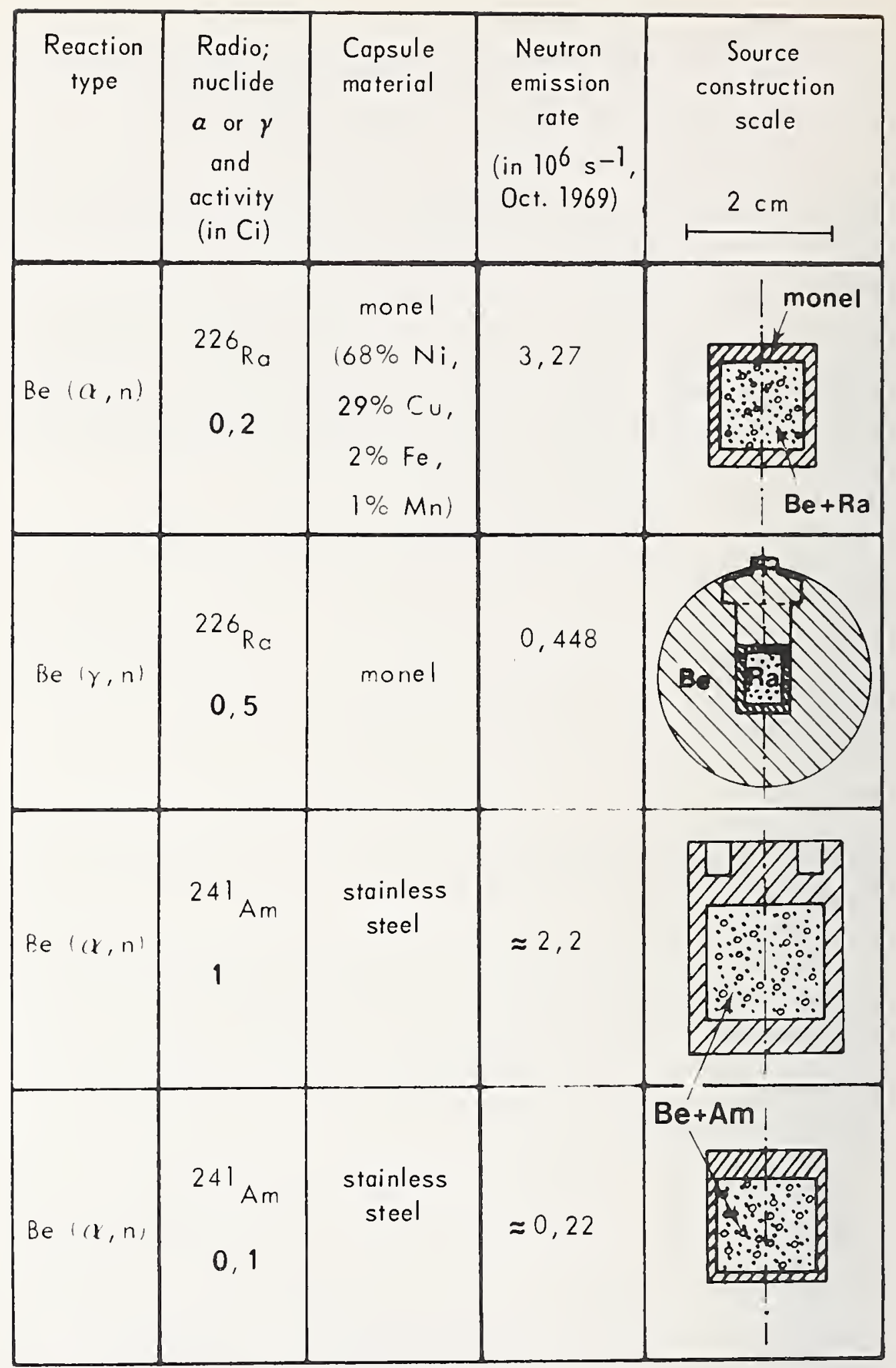

Figure 1. The BIPM neutron sources. 
the neutron loss is of the order of 1 percent; for the $\operatorname{Ra}-\mathrm{Be}(\gamma, \mathrm{n})$ source, whose spectrum extends up to a maximum energy of $700 \mathrm{keV}$, a $50 \mathrm{~cm}$ diameter sphere is used and the loss of neutrons is negligible. If a neutron is captured by a nucleus of ${ }^{55} \mathrm{Mn}$, the nucleus is transformed into ${ }^{56} \mathrm{Fe}$, according to the scheme:

$$
{ }^{55} \mathrm{Mn}+\mathrm{n} \rightarrow{ }^{56} \mathrm{Mn} \stackrel{\beta}{\rightarrow}{ }^{56} \mathrm{Fe}^{*} \stackrel{\gamma}{\rightarrow} 56 \mathrm{Fe}
$$

where the asterisk indicates an excited state. If a neutron is captured by a hydrogen nucleus, a deuteron (stable) is formed. Capture by other atomic nuclei present in the solution is much less frequent.

The disintegration scheme of ${ }^{56} \mathrm{Mn}$ is represented in figure 2. The emission rate for neutrons from the source $Q$ is equal to the sum of the capture rates for neutrons in the solution and in the source (self-absorption), and of the rate of escape of neutrons from the solution. One thus has

$$
Q=Q_{\mathrm{Mn}}(1 / f) k,
$$

where $Q_{\mathrm{Mn}}$ is the number of neutrons captured per second by the manganese nuclei in the solution, $f$ is the ratio of the number of neutrons captured by manganese nuclei to the number of neutrons captured by the totality of elements contained in the solution $(f \approx 0.5), k$ is a correction factor to allow for systematic errors due to the absorption of neutrons (especially slow ones) in the source due to the presence of the bath, to the ab-

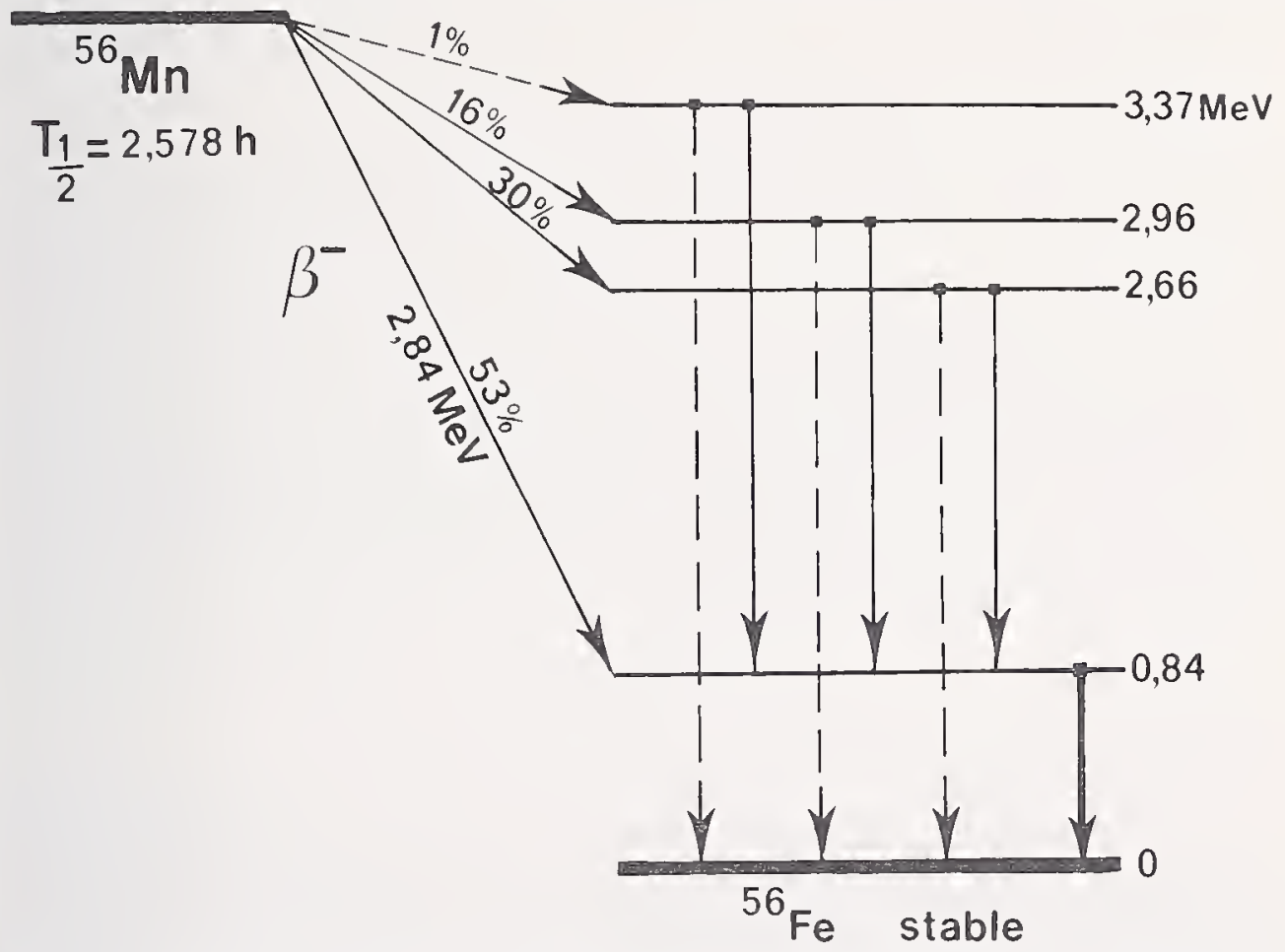

Figure 2. Disintegration scheme for ${ }^{56} \mathrm{Mn}$. The nucleus transforms itself by emission of a $\beta^{-}$ particle (oblique arrows) into ${ }^{56} \mathrm{Fe}$ in an excited state: the latter then loses its excess energy by emitting a photon of $\gamma$ radiation (vertical arrows). The horizontal lines correspond to the energy levels of the nuclei. 
sorption of fast neutrons by oxygen and sulfur in the solution, to impurities in the solution, and to the escape of neutrons from the manganese bath $(k \approx$ 1.04).

For irradiation to saturation, that is to say for an irradiation time $t$ long in comparison to the half-life of ${ }^{56} \mathrm{Mn}\left(T_{1 / 2} \approx 2.6\right.$ hours $)$, as many neutrons are being captured by the ${ }^{55} \mathrm{Mn}$ as there are ${ }^{56} \mathrm{Mn}$ nuclei disintegrating, and the disintegration rate $Q^{\prime}{ }_{M n}$, at the moment the source is withdrawn from the solution (time $t^{\prime}=0$ ), is equal to $Q_{\mathrm{Mn}}$. In practice, the source is withdrawn at the end of a time $t \approx 16 \mathrm{~h}$, and at time $t^{\prime}=0$ we have:

$$
Q_{\mathrm{Mn}}^{\prime}(t)=Q_{\mathrm{Mn}}\left(1-\mathrm{e}^{-\lambda t}\right)=0.9865 Q_{\mathrm{Mn}}
$$

where $\lambda=\ln 2 / T_{1 / 2}=0.2688 \mathrm{~h}^{-1}$.

In order to measure the relative activity of the irradiated solution, $\gamma$ rays emitted by the ${ }^{56} \mathrm{Fe}^{*}$ are counted by means of a scintillation detector (sodium iodide crystal) immersed in the bath in place of the source. This relative measurement of activity is completed by an absolute calibration: after the bath has lost its activity, a quantity of active ${ }^{56} \mathrm{Mn}$ solution, whose specific activity has been previously determined by the method of $4 \pi \beta-\gamma$ coincidence counting is added to the bath. We then have

$$
Q_{\text {Mn }}^{\prime}=A\left(\rho_{\text {source }}\right) /\left(\rho_{\text {solution }}\right) \text {, }
$$

where $\rho_{\text {source }}$ designates the counting rate obtained in the irradiated manganese bath, and $\rho_{\text {solution }}$ is the rate obtained after adding a solution of activity $A$ to the bath.

For measurement of $\operatorname{Ra-Be}(\gamma, n)$ sources, we have also set up a variant of the manganese bath method. It involves measuring the activity of the manganous sulfate solution outside the sphere in which the solution is continuously irradiated by the source to be measured. A pump circulates the solution in a closed circuit (flow of 6 litres per minute) between the sphere and an enclosure 10 metres away (fig. 3). This enclosure is protected from $\gamma$-rays from the source by a 40 -cm thick concrete wall and by a lead shield with wall thickness of $10 \mathrm{~cm}$; in this shielded location the measurements of the activity of the irradiated solution are made. This new technique, called the circulation method, lends itself particularly well to the measurement of relatively low intensity neutron sources.

\section{Comparative Measurements of Neutron Sources}

It is planned that the neutron sources belonging to the BIPM remain there permanently. National laboratories which desire to have their own sources compared can send them to the BIPM; the BIPM can however organize international comparisons using sources furnished by other laboratories, as in the following example. 


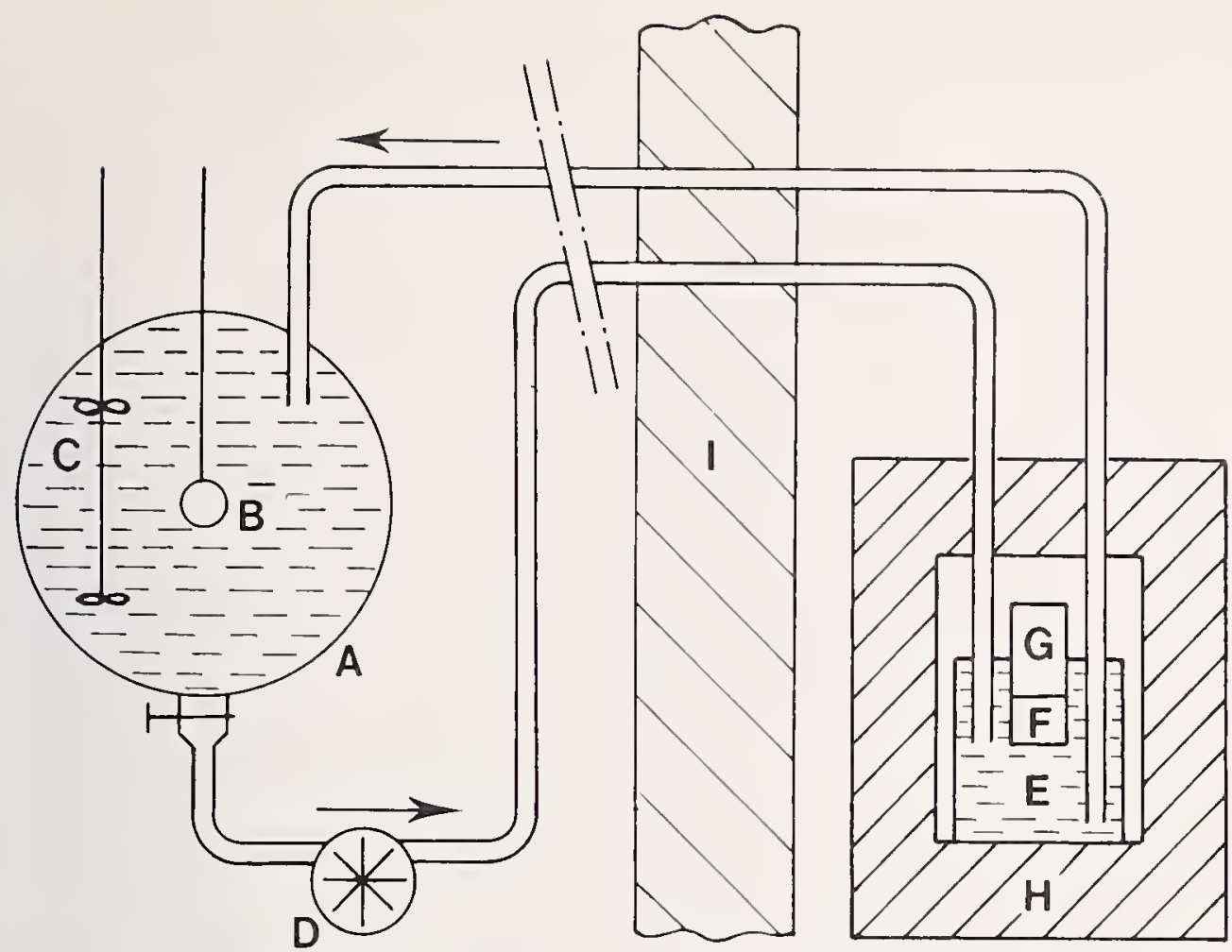

Figure 3. Measurement of the emission rate of a neutron source by the method of circulation of a manganous sulfate solution. A: sphere $(d=50 \mathrm{~cm})$ containing the solution: $B:(\gamma, n)$ source; C: stirrers; D: circulation pump; $\mathrm{E}$ : counting beaker placed $10 \mathrm{~m}$ from the sphere; F: sodium iodide crystal; G: photomultiplier; H: lead shield (wall thickness : $10 \mathrm{~cm}$ ); I: concrete wall (thickness : $40 \mathrm{~cm}$ ).

Standard Ra-Re $(\alpha, n)$ Source No. 200-1 of the National Research Council of Canada

Measurements of the emission rate of this source, made by the manganese bath method from January 1962 to March 1965 by 10 participating laboratories [1], gave a 3 percent maximum spread among the results. The total uncertainty estimated by the participants varied between \pm 0.7 and \pm 1.7 percent (fig. 4). The manganese bath method, adopted by most of these laboratories, is considered to be the method capable of the best precision in measuring the emission rate of a neutron source.

\section{Standard Ra-Be $(\alpha, n)$ and Ra-Be $(\gamma, n)$ Sources of the BIPM}

The standard Ra-Be $(\alpha, n)$ source (fig. 1) has been measured periodically at the BIPM since December 1963, by the manganese bath method; the consistency of the measurements is very satisfactory. Currently, the reproducibility of measurement is 0.1 percent, while the accuracy of the emission rate of the source is 1 percent. The principal sources of error are 


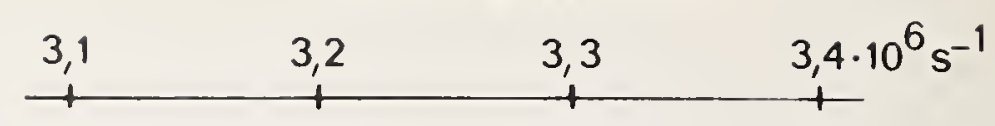

ETL

IKO

CENS

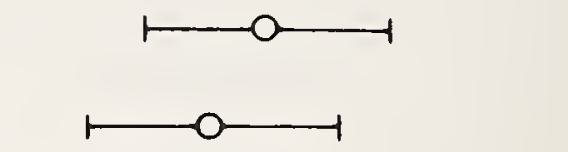

IMM

SRE

BCMN

BIPM
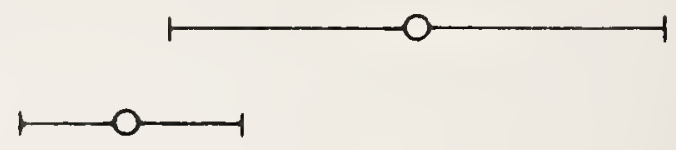

AEET

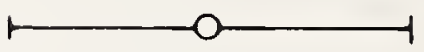

NRC

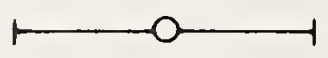

NBS
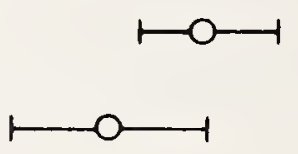

NPL

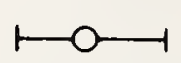

mean value

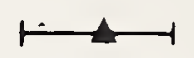

Figure 4. Results of measurements of emission rate of the Ra-Be $(\alpha, n)$ neutron source No. 200-1 of the NRC, normalized to reference cross sections and to January 1962. The laboratories are listed in the order of the dates of measurement (January 1962 to February 1965). The measurement by the NPL dates from June 1959. The bars indicate the total uncertainties estimated by the participants.

the uncertainties in the capture cross sections for thermal and fast neutrons, and in the correction factors.

As for the standard $\operatorname{Ra}-\operatorname{Be}(\gamma, n)$ source (fig. 1), the emission factor has been determined with an accuracy of 0.5 percent. In fact, the $\operatorname{Ra-Be}(\gamma, n)$ sources have the advantage over the $\operatorname{Ra}-\mathrm{Be}(\alpha, n)$ sources of not needing correction for absorption by the $(n, p)$ and $(n, \alpha)$ reactions with oxygen and sulfur, the upper neutron energy limit of $700 \mathrm{keV}$ being below the threshold for these reactions. 


\section{Measurement of the Flux Density of a Source of Fast Monoenergetic Neutrons}

The BIPM has undertaken the study of a neutron source using the reaction ${ }^{2} \mathrm{H}(\mathrm{d}, n)^{3} \mathrm{He}$. This source, which emits relatively monoenergetic fast neutrons of $2.5 \mathrm{MeV}$, can in fact be studied with rather modest experimental facilities; the source also emits, in association with the neutrons, charged ${ }^{3} \mathrm{He}$ particles which can be detected in order to measure the neutron flux density in a given direction by the associated particle method.

The principle of this measurement method is the following: the emission of each neutron corresponds to the emission of a ${ }^{3} \mathrm{He}$ particle in the opposite direction in the center-of-mass system ${ }^{3}$ in other words, in the system of the center-of-mass, the number of ${ }^{3} \mathrm{He}$ particles emitted per unit solid angle in a given direction must be equal to the number of neutrons emitted per unit solid angle in the opposite direction. It is therefore possible to measure the neutron flux density in a given direction either by counting the ${ }^{3} \mathrm{He}$ particles (with an efficiency of $100 \%$ ) by means of a surface-barrier semiconductor detector, or by the counting of neutrons by means of a scintillation counter (hydrogenous scintillator) whose efficiency has been measured. These two relatively independent methods can be used simultaneously to compare the results obtained.

Let us designate by $\Phi_{\mathrm{He}}$ the number of neutrons measured by counting the ${ }^{3} \mathrm{He}$ particles in the solid angle $\Omega_{\mathrm{n}}$ covered by the neutron detector. If $k_{\mathrm{He}}$ and $k_{\mathrm{n}}$ are the factors for converting solid angles from the laboratory system $(\Omega)$ to the center-of-mass system $\left(\Omega^{\prime}\right)$, for ${ }^{3} \mathrm{He}$ particles and neutrons respectively, and if $N_{\mathrm{He}}$ is the total number of ${ }^{3} \mathrm{He}$ particles counted with the use of the semiconductor detector which covers the solid angle $\Omega_{\mathrm{He}}$, we can write:

$$
\begin{gathered}
\Omega_{\mathrm{n}}=k_{\mathrm{n}} \Omega_{\mathrm{n}}^{\prime}, \Omega_{\mathrm{He}}=k_{\mathrm{He}} \Omega_{\mathrm{He}}^{\prime}, \\
\Phi_{\mathrm{He}}=N_{\mathrm{He}} \frac{\Omega_{\mathrm{n}}}{\Omega_{\mathrm{He}}} \frac{k_{\mathrm{He}}}{k_{\mathrm{n}}} .
\end{gathered}
$$

On the other hand, if $\Phi_{\mathrm{n}}$ represents the number of neutrons emitted in $\Omega_{\mathrm{n}}$ and $N_{\mathrm{n}}$ the number of neutrons counted by the hydrogen scintillation counter of efficiency $\epsilon$, we can write

$$
\Phi_{\mathrm{n}}=\left(N_{\mathrm{n}} / \epsilon\right) f
$$

The factor $f$ takes account of effects such as: neutron scattering, anisotropy of the source, emission of neutrons by secondary sources, etc. We determine the efficiency $\epsilon$ in the course of the same series of measurements, by counting, in addition to $N_{\mathrm{He}}$ and $N_{\mathrm{n}}$, the coincidences of ${ }^{3} \mathrm{He}$ par-

${ }^{3}$ The center-of-mass system is the reference system in which the center of mass of all the particles participating in the reaction is taken as the origin. This system is not at rest relative to the laboratory frame-of-reference, but it has the advantage of making the formulas particularly simple for calculating speeds and angles. 


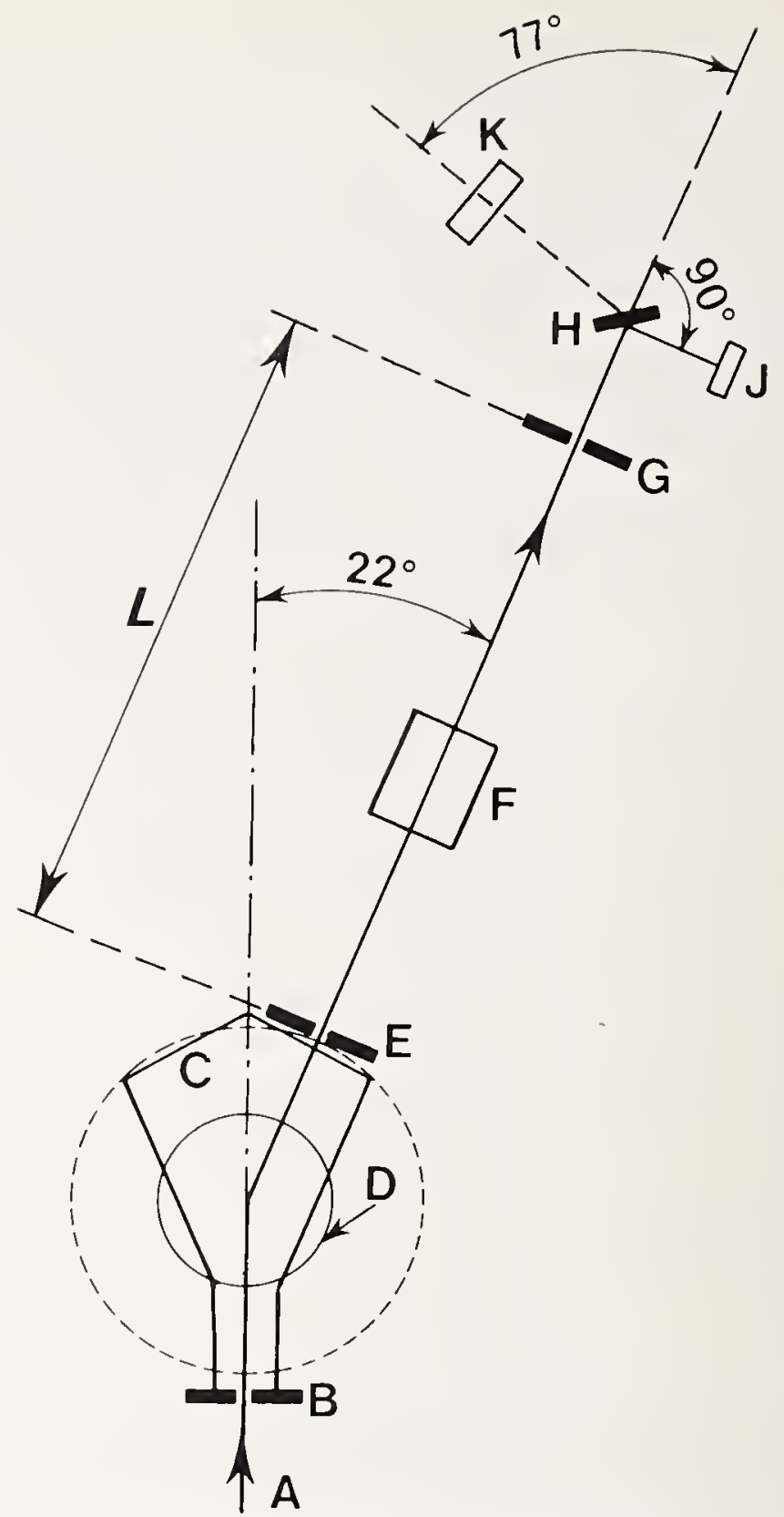

Figure 5. Diagram of experimental arrangement for measurement of the flux density of a ${ }^{2} \mathrm{H}(\mathrm{d}, \mathrm{n})^{3} \mathrm{He}$ neutron source; comparison of the two methods (direct detection and associated particle). A: beam of accelerated deuterons $(100 \mathrm{keV}) ; \mathrm{B}$ : aperture, $\mathrm{d}=12 \mathrm{~mm}$; $\mathrm{C}$ : vacuum chamber of magnetic analyzer; D: magnetic field; E: aperture, $\mathrm{d}=10 \mathrm{~mm}$ : $\mathrm{F}$ : quadrupole electrostatic lens: $\mathrm{G}$ : aperture, $\mathrm{d}=5 \mathrm{~mm} ; \mathrm{H}$ : target (neutron source) of deuterated titanium: J: semiconductor detector for ${ }^{3}$ He particles: K: hydrogenous scintillator, neutron detector; $\mathrm{L}$ : distance of $1.2 \mathrm{~m}$.

The ensemble $B$ to $G$ serves to eliminate molecular ions from the beam and to concentrate the deuterons on the target by magnetic separation. When an angle of $90^{\circ}$ is chosen for observation of ${ }^{3} \mathrm{He}$ particles, the neutron detector must be located at $77^{\circ}$ to count the neutrons associated with these particles. 
ticles and neutrons. Finally, it is a matter of comparing the two quantities $\Phi_{\mathrm{He}}$ and $\Phi_{\mathrm{n}}$ which should be equal.

Figure 5 shows the experimental arrangement which includes an electrostatic accelerator $(150 \mathrm{kV}, 2 \mathrm{~mA})$. Experiments have been carried out $[2,3]$ with a beam of $100 \mathrm{keV}$ deuterons and a target current of $2 \mu \mathrm{A}$ (emis sion rate of the source $\approx 10^{5} \mathrm{~s}^{-1}$ ), observing the ${ }^{3} \mathrm{He}$ particles at $150^{\circ}, 120^{\circ}$, and $90^{\circ}$ (in the laboratory reference system). The flux densities of neutrons of $2.76 \mathrm{MeV}, 2.67 \mathrm{MeV}$, and $2.53 \mathrm{MeV}$ have been measured by each of the two detectors with an uncertainty of \pm 2 percent. In comparing the values of $\Phi_{\mathrm{He}}$ and $\Phi_{\mathrm{n}}$, we have been able to determine that when the target is perpendicular to the direction of the incident deuteron beam (observation of ${ }^{3} \mathrm{He}$ particles at $150^{\circ}$ and $120^{\circ}$ ), there has been a satisfactory agreement between $\Phi_{\mathrm{He}}$ and $\Phi_{n}$; on the contrary, a disagreement has appeared $\left(\Phi_{n}>\right.$ $\Phi_{\mathrm{He}}$ ) when the target is inclined at $45^{\circ}$ (differences of +4.2 percent for the observation of ${ }^{3} \mathrm{He}$ particles at $90^{\circ}$ and of +3.1 percent for observation at $120^{\circ}$ ); none of the interpretations considered until now to explain this dis crepancy has been satisfactory.

To compare measurements of neutron flux density among various laboratories, the use of rather simple and rugged, but relatively insensitive, transfer instruments is anticipated. The participation of the BIPM in such comparisons being highly desirable, a considerable increase in the emission rate of the ${ }^{2} \mathrm{H}(\mathrm{d}, \mathrm{n})^{3} \mathrm{He}$ neutron source has become indispensable. With an incident deuteron energy of $140 \mathrm{keV}$ and a target current of $200 \mu \mathrm{A}$ (target cooled by a jet of nitrogen whose temperature is slightly below $0{ }^{\circ} \mathrm{C}$ ), it has been possible to increase the maximum emission rate for neutrons from this source to $4 \times 10^{7} \mathrm{~s}^{-1}$ in the solid angle $4 \pi$ steradian [2].

The results obtained with the lower emission rate, although not yet satisfactory, seem to indicate that the absolute measurement of a ${ }^{2} \mathrm{H}(\mathrm{d}, \mathrm{n})^{3} \mathrm{He}$ neutron source by the associated particle method does not pose insoluble problems. The future should show if the precision and consistency of these measurements can be increased enough that the results can be comparable to those of measurements of the same source by a neutron slowing-down method. 


\section{Bibliography}

[1] Naggiar, V., Rapport sur la comparaison internationale de la mesure du taux d'émission de la source de neutrons Ra-Be $(\alpha, n)$ du Conseil National de Recherches No. 200-1 par la méthode de ralentissement des neutrons dans une solution de sulfate de manganèse (fevrier 1967), Recueil de Travaux du B.I.P.M. 1, 48 pages (1966-1967).

[2] Huynh, V. D., Lafaye, L., and Bréonce, P., dans Procès-Verbaux C.I.P.M. 40, pp. 75-82 (1972).

[3] Naggiar, V., Lafaye, L., and Brćonce, P., Distribution angulaire d'un€ source de neutrons $\mathrm{D}(\mathrm{d}, \mathrm{n})^{3} \mathrm{He}$ en cible ćpaisse pour deutons de $100 \mathrm{keV}$, Nucl. Instr. and Meth. 41, pp. $77-83$ (1966). 


\section{Appendix 1}

\section{Convention of the Metre}

(May 20, 1875)

His Excellency the President of the French Republic, His Majesty the Emperor of Germany, His Majesty the Emperor of Austria-Hungary, His Majesty the King of the Belgians, His Majesty the Emperor of Brazil, His Excellency the President of the Argentine Confederation, His Majesty the King of Denmark, His Majesty the King of Spain, His Excellency the President of the United States of America, His Majesty the King of Italy, His Excellency the President of the Republic of Peru, His Majesty the King of Portugal and the Algarves, His Majesty the Emperor of all the Russias, His Majesty the King of Sweden and Norway, His Excellency the President of the Swiss Confederation, His Majesty the Emperor of the Ottomans, and His Excellency the President of the Republic of Venezuela, desiring international uniformity and precision in standards of weight and measure, have resolved to conclude a convention to this effect, and have named as their plenipotentiaries the following:

His Excellency the President of the United States of America: Mr. Elihu Benjamin Washburne, Envoy Extraordinary and Minister Plenipotentiary of the United States at Paris;

His Majesty the Emperor of Germany: His Highness Prince Hohenlohe Schillingsfürst, Grand Cross of the Order of the Red Eagle of Prussia, and of the Order of St. Hubert of Bavaria, \&c., \&c., \&c., his Ambassador Extraordinary and Plenipotentiary at Paris;

His Majesty the Emperor of Austria-Hungary: His Excellency Count Apponyi, his current Chamberlain and Privy Counselor, Knight of the Golden Fleece, Grand Cross of the Royal Order of St. Stephen of Hungary, and of the Imperial Order of Leopold, \&c., \&c., \&c., his Ambassador Extraordinary and Plenipotentiary at Paris;

His Majesty the King of the Belgians: Baron Beyens, Grand Officer of his Order of Leopold, Grand Officer of the Legion of Honor, \&c., \&c., \&c., his Envoy Extraordinary and Minister Plenipotentiary at Paris;

His Majesty the Emperor of Brazil: Mr. Marcos Antonio d'Araujo, Viscount d'Itajuba, Grandee of the Empire, member of His Majesty's Council, Commander of his Order of Christ, Grand Officer of the Legion of

\footnotetext{
${ }^{1}$ Articles preceded by the sign $>$ have been modified, or supplemented, by the International Convention of 1921 (see p. 230).
} 
Honor, \&c., \&c., \&c., his Envoy Extraordinary and Minister Plenipotentiary at Paris:

His Excellency the President of the Argentinc Confederation: Mr. Balcarce, Envoy Extraordinary and Minister Plenipotentiary of the Argentine Confederation at Paris;

His Majesty the King of Denmark: Count de Moltke-Hvitfeldt, Grand Cross of the Order of Dannebrog, and decorated with the Cross of Honor of the same order, Grand Officer of the Legion of Honor, \&c., \&c., \&c., his Envoy Extraordinary and Minister Plenipotentiary at Paris;

His Majesty the King of Spain: His Excellency Don Mariano Roca de Togores, Marquis de Molins, Viscount de Rocamora, Grandee of Spain of the First Class, Knight of the Renowned Order of the Golden Fleece, Grand Cross of the Legion of Honor, \&c., \&c., \&c., Director of the Royal Spanish Academy, his Ambassador Extraordinary and Plenipotentiary at Paris; and

General Ibañez, Grand Cross of the Order of Isabella the Catholic, \&c., \&c., Director General of the Geographical and Statistical Institute of Spain, Member of the Academy of Sciences;

His Excellency the President of the French Republic: The Duke Decazes, deputy to the National Assembly, Commander of the Order of the Legion of Honor, \&c., \&c., \&c., Minister of Foreign Affairs;

the Viscount de Meaux, deputy to the National Assembly, Minister of Agriculture and of Commerce; and

Mr. Dumas, Perpetual Secretary to the Academy of Sciences, Grand Cross of the Order of the Legion of Honor;

His Majesty the King of Italy: The Chevalier Constantin Nigra, Knight of the Grand Cross of his Orders of St. Maurice and St. Lazarus, and of the Crown of Italy, Grand Officer of the Legion of Honor, \&c., \&c., \&c., his Envoy Extraordinary and Minister Plenipotentiary at Paris;

His Excellency the President of the Republic of Peru: Mr. Pedro Galvez, Envoy Extraordinary and Minister Plenipotentiary of Peru at Paris; and Mr. Francisco de Rivero, formerly Envoy Extraordinary and Minister Plenipotentiary of Peru;

His Majesty the King of Portugal and of the Algarves: Mr. José da Silva Mendes Leal, Peer of the Realm, Grand Cross of the Order of Saint James, Knight of the Order of the Tower and Sword of Portugal, \&c., \&c., \&c., his Envoy Extraordinary and Minister Plenipotentiary at Paris;

His Majesty the Emperor of all the Russias: Mr. Gregory Okouneff, Knight of the Russian Orders of St. Anne of the first class, of St. Stanislaus of the first class, of St. Vladimir of the third, Commander of the Legion of Honor, current Counselor of State, Counselor of the Embassy of Russia at Paris;

His Majesty the King of Sweden and Norway: Baron Adelswärd, Grand Cross of the Order of the Polar Star of Sweden, and of St. Olaf of Norway, Grand Officer of the Legion of Honor, \&c., \&c., \&c., his Envoy Extraordinary and Minister Plenipotentiary at Paris; 
His Excellency the President of the Swiss Confederation: Mr. Jean Conrad Kern, Envoy Extraordinary and Minister Plenipotentiary of the Swiss Confederation at Paris;

His Majesty the Emperor of the Ottomans: Husny Bey, LieutenantColonel of Staff, wearer of a fourth-class decoration of the Imperial Order of Osmania, of a fifth-class decoration of the Order of Medjidie, Officer of the Legion of Honor, \&c., \&c., \&c.;

His Excellency the President of the Republic of Venezuela: Doctor Eliseo Acosta,

Who, after having exhibited their full powers, which were found to be in good and due form, have agreed upon the following articles:

\section{Article 1.}

The high contracting parties engage to establish and maintain, at their common expense, a scientific and permanent international bureau of weights and measures, the location of which shall be at Paris.

\section{Article 2.}

The French Government shall take all the necessary measures to facilitate the purchase, or, if expedient, the construction, of a building which shall be especially devoted to this purpose, subject to the conditions, stated in the regulations which are subjoined to this convention.

\section{ARTICLE 3.}

The operation of the international bureau shall be under the exclusive direction and supervision of an international committee for weights and measures, which latter shall be under the control of a general conference on weights and measures, to be composed of the delegates of all the contracting governments.

\section{Article 4.}

The general conference on weights and measures shall be presided over by the president for the time being of the Paris Academy of Sciences.

\section{ARticle 5.}

The organization of the bureau, as well as the formation and the powers of the international committee, and of the general conference on weights and measures, are established by the regulations subjoined to this convention. 
The international bureau of weights and measures shall be charged with the following duties:

1st. All comparisons and verifications of the new prototypes of the metre and kilogram.

$2 d$. The custody of the international prototypes.

$3 \mathrm{~d}$. The periodic comparison of the national standards with the international prototypes and with their test copies, ${ }^{2}$ as well as comparisons of the standard thermometers.

4th. The comparison of the prototypes with the fundamental standards of non-metric weights and measures used in different countries for scientific purposes.

5th. The sealing and comparison of geodetic measuring-bars.

6th. The comparison of standards and scales of precision, the verification of which may be requested by governments or by scientific societies, or even by constructors or men of science.

\section{$\checkmark$ Article 7.}

The persons composing the bureau shall be a director, two assistants, and the necessary number of employees.

When the comparisons of the new prototypes shall have been finished, and when these prototypes shall have been distributed among the different states, the number of persons composing the bureau shall be reduced so far as may be deemed expedient.

The governments of the high contracting parties will be informed by the international committee of the appointment of the persons composing this bureau.

\section{- ARTicle 8.}

The international prototypes of the metre and of the kilogram, together with the test copies² of the same, shall be deposited in the bureau, and access to them shall be allowed to the international committee only.

\section{Article 9.}

The entire expense of the construction and outfit of the international bureau of weights and measures, together with the annual cost of its maintenance and the expenses of the committee, shall be defrayed by contributions from the contracting states, the amount of which shall be computed in proportion to the actual population of each.

"Editor's note: Modern terminology is "check standards." 
The amounts representing the contributions of each of the contracting states shall be paid at the beginning of each year, through the ministry of foreign affairs of France, into the Caisse de dépots et consignations at Paris, whence they may be drawn as occasion may require, upon the order of the director of the bureau.

\section{- ARTICLe 11.}

Those governments which may take advantage of the privilege, open to every state, of acceding to this convention, shall be required to pay a contribution, the amount of which shall be fixed by the committee on the basis established in article 9, and which shall be devoted to the improvement of the scientific apparatus of the bureau.

\section{ARTiCle 12.}

The high contracting parties reserve to themselves the power of introducing into the present convention, by common consent, any modifications the propriety of which may have been shown by experience.

\section{ARTiCle 13.}

At the expiration of 12 years this convention may be abrogated by any one of the high contracting parties, so far as it is concerned.

Any government which may avail itself of the right of terminating this convention, so far as it is concerned, shall be required to give notice of its intentions one year in advance, and by so doing shall renounce all rights of joint ownership in the international prototypes and in the bureau.

\section{ARTICLE 14.}

This Convention shall be ratified according to the constitutional laws of each state, and the ratifications shall be exchanged in Paris within 6 months, or sooner, if possible. It shall take effect on the first day of January 1876.

In testimony whereof the respective plenipotentiaries have attached their signatures and have hereunto affixed their seals of arms.

Done at Paris, May 20, 1875. (The seals and signatures of the plenipotentiaries follow.) 
This Convention was modified slightly by the international Convention signed at Sèvres on October 6, 1921.

Article 7 became:

After the Committee shall have proceeded with the work of coordinat ing the measures relative to electric units and when the general conference shall have so decided by a unanimous vote, the Bureau will have charge of the establishment and keeping of the standards of the electric units and their test copies and also of comparing with those standards, the national or other standards of precision.

The Bureau is also charged with the duty of making the determinations relative to physical constants, a more accurate knowledge of which may be useful in increasing precision and further insuring uniformity in the provinces to which the above mentioned units belong (Article 6 and 1st paragraph of Article 7).

It is finally charged with the duty of coordinating similar determinations effected in other institutions.

In Article 8, the words "of the metre and of the kilogram" were deleted.

Article 11 was supplemented as follows:

Any state may adhere to this convention by giving notice thereof to the French Government which shall notify all the participant states and the Chairman of the International Committee for Weights and Measures.

Any new accession to the Convention of May 20, 1875, will necessarily involve adherence to this Convention.

Regulations are annexed to the Convention of 1875; they have the same force and value as the Convention itself. These regulations, which were modified in 1907, 1921, and 1960, establish in particular:

- Requirements on construction, equipment, and operation of the BIPM (buildings, instruments, budgetary rules).

- The role of the CGPM (dissemination and improvement of the metric system, ratification of fundamental metrological determinations, election to the CIPM), the voting rules and the distribution of financial expenses among the States.

- The composition, renewal procedures, and operation of the CIPM as well as its mission (direction of the work and operations of the BIPM, nomination of the director and some assistants, establishment of the budget, setting calibration fees, surveillance of the prototypes, annual report to the Governments).

- The conditions for access to the place of deposit of the prototypes and of publication (in French) of reports and work of the BIPM and CIPM. 
Appendix 2

\title{
Member States of the Metre Convention 1875 Signatories
}

\author{
Argentina \\ Austria-Hun grary \\ Belgium \\ Brazil \\ Denmark \\ France \\ Germany \\ Italy \\ Регu*
}

\author{
Porluyal \\ Russia \\ Spain \\ Sweden and Norway \\ Swilzerland \\ Turkey \\ U.S.A. \\ Venezuela
}

\section{Member States as of January 1,1975 (43 States) and Dates of Adherence}

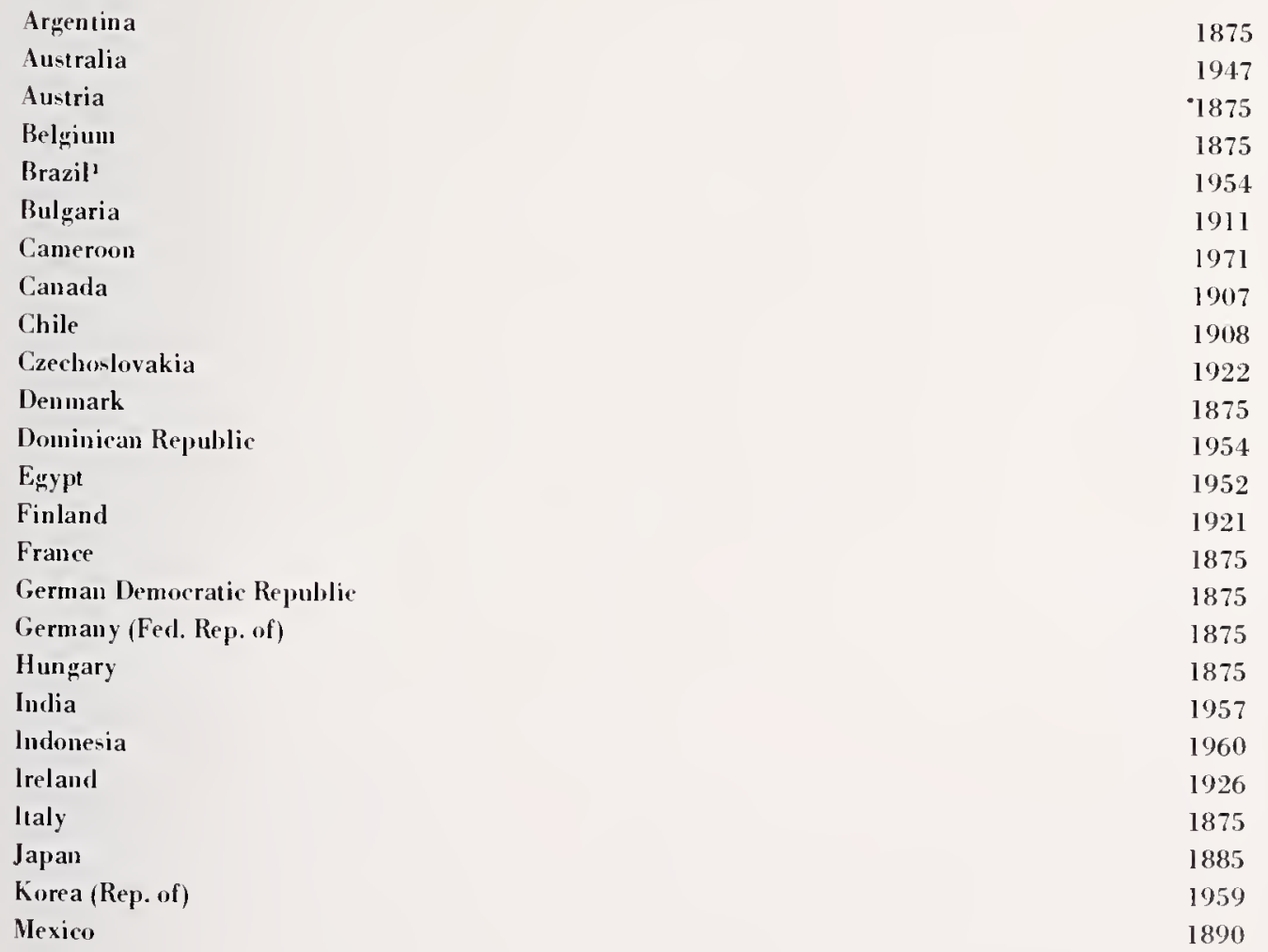

German Democratic Republic

India

lndonesia

Japan 
Vetlerlands (The)

Pakislan

1973

Poland

1925

Portugal

187.5

Romania

188.3

Spain

187.5

Sonth Ifrica

1961

swerlen

1875

switererland

187.5

Thailand

1912

Turkey ${ }^{2}$

193.3

1875

188.4

United Kingrlom

1875

1908

1960

lomoliaia

1879

\footnotetext{
${ }^{3}$ Turkey. an 1875 -innalory withdres in 1889.

3 signatory in 1875 . artherence nol mainlained in 1906.
} 


\section{Members and Officers of the CIPM, and Directors of the BIPM from 1875 to 1975 (in chronological order)}

\section{Members of the CIPM}

One hundred and ten noted reientists have served on the CIPII since its inception.

Bourcha. J.. Delft, The Vetherlands.'

1875

Broch, O. J., Christiana, Norway

$1875-1889$

Chisholm, H, W', London. Great Britain'

1875

Foerster, W., Berlin, Germany

1875-1921

Govi, G.. Turin, Italy

$1875-1889$

Herr, J., Vienna, Austria

Hilgard, J. E., Washington. D.C., U.S.A.

1875-1884

Hirsch, A, Neuchatel, Switzerland

Husny-Bey, Constantinople, Turkey

Ibañez, C., Madrid, Spain

$1875-1887$

1875-1901

Morin, A., Paris. France

$1875-1883$

1875-1891

$1875-1880$

Stas, J.-S, Brumels, Bel wiun

Wild, H., St. Petersburg, Russia

1875-1891

Wrede, Baron de, Stockholm, Sweden

1875-1895

1875-1885

Gould, B.-A.. Cordova, Argentina

$1879-1884$

Kruspér, E. de, Pest, Hun gary

1879-1894

Dumas, J.-B., Paris, France

1880-1884

Aguiar, A. de, Lisbon, Portugal

1884-1887

Bertrand, J., Paris, France

1884-1900

Christie, W.-II., Greenwich, Great Britain

1885-1891

Oppolzer, Th, son. Vicmua, Austria

Thalén, R., Uppsala, Sweden

$1885-1886$

Gould, B.-A., Cambridge, U.S.A.

$1885-1900$

$1887-1896$

Lang, V. von. Vienna, Austria

1887-1921

Macedo, H. de, Lisbon, Portugal

1887-1901

Arndsten, A.. Christiana. Norway

$1889-1913$

Brioschi, J., Rome, ltaly

1889-1895

Chaney. H.-J.. London, Great Britain

1891-1906

Arrillaga, F. de P., Madrid, Spain

1894-1920

Bodola, L. de, Budapest, Hungary

1894-1929

Hepites, St.-C., Bucharent, Romania

1894-1922

Ferraris, G., Turin, ltaly

1895-1897

Mendeleyev, D., St. Petersburg, Russia

1895-1901 "The Vetherland and Great Britain not haring signed the Vetre Comention in 1875. J. Bumcha and H. H. (hi-holm re-ignet from the
CIPY. 
Egroroff, N.. St. Petersburg, Russia

Gantier, R., Geneva, Switzerland

Mascart, E.. Paris, France

Stratton, S. W., Boston. U.S.A.

1905-1931

Gill, Sir David, London, Great Britain 1907-1914

Tanakarlate, A.. Tokyo, Japan 1907-1931 Darboux, J.-G., Paris, France 1909-1917

Appell, P.. Paris, France

Macllahon, P.A., London, Great Britain

1919-1930

Pasquier, E.. Louvain, Belgrum

Volterra, V., Rome, Italy

Kösters, W., Berlin, Germany

Torres y Quevedo, L., Madrid, Spain

Isaachsen, D., Oslo, Norway

1919-1929

1919-1926

1919-1940

1921-1950

1921-1929

1922-1940

1923-1927

Fredholm, E.-I.. Stockholm, Sweden

1923-1965

Karyatchin, C.. Belirarle. Yugonlavia

1927-1929

Konovalov, D., Leningrad, U.S.S.R.

Cabrera, B., Madrid, Spain

Johansen, E. S., Copenhagen, Denmark

1928-194.1

MaeLenan, J. C., Toronto, Canada

1928-1952

1928-1935

Posejpal. V.. Praque, Czeehoslovakia

1928-1935

Chatelain, M.. Lelingrad. U.S.S.R.

1929-1948

Stateseu. C., Buelıarest, Romania

Zeeman, P., Amsterdan, The Netherlands

1929-1958

Sears, J. E., Teddington-London, United Kingdom

1929-1943

1930-1954

Janet, P., Paris, Franee

Nagaoka, H., Tokyo, Japan

1931-1937

1931-1948

1933-1939

Kennelly, A. E., Cambridye, U.S.A.

1933-1954

Roร, M. G., Zurich, Switzerland

1936-1952

Dehalu, M., Liege, Belgium

1936-1945

Rauszer, Z., Varsovy, Poland

$1937-1945$

Fabry, C., Paris, France

1939-1959

Siegbahn, M., Uppsala, Sweden

1946-1951

de Broglie, L., Paris, Franee

1946-1964

Cassinis, G., Milan, Italy

Crittenden, E. C., Washington, D.C., U.S.A.

1946-1954

de Haas, W. J., Leyden, The Netherlands

1946-1951

Isnardi, T., Buenos Aires, Argentina

Field, R. H., Ottawa, Canada

1951-1954

1951-1954

Kouznetsov, A., Moseow, U.S.S.R.

$1952-1964$

Danjon, A., Paris, France

Vieweg, R., Braunsehweig, Germany (Fed. Rep. of)

1952-1965

Yamauti, Z., Tokyo, Japan

1952-1967

de Boer, J., Amsterdam, The Netherlands*

1953-

Nussberger, J., Prague, Czechoslovakia

1953-1970

1954-1969

Astin, A. V., W ashington, D.C., U.S.A.

1954-1966

Barrell, H., Teddington-London, United Kingdom

1954-1966

Bourdoun, G. D., Moseow, U.S.S.R.

Esserman, N. A., Chippendale-Sydney, Australia

1954-1962

Otero, J. M., Madrid, Spain* 
Stulla-Götz. J., Vienna. Anstria

Våisälä, Y., Turku, Finland

Howlett, L. E., Ottawa, Canada

1955-1969

Krishnan, K. S., New Delhi, India

1958-1961

Sandoval Vallarta, M., Mexico City, Mexico*

$1960-$

Lehany, F. J., Chippendale-Sydney, Australia*

$1963-$

Kichlu, P. K., New Dellui, ludia

Maréchal, A., Paris, France*

Siegbahn, K., Uppsala, Sweden*

Kersten. M.. Braun-chweig, Germany (Fed. Rep. of)

$1964-1966$

1964-

1964-

$1965-1970$

1966-

Niewodniczanski, H., Krakow, Poland

1966-1968

Novikov, l. l., Moscow, U.S.S.R.

1966-1969

Cintra do Prado, L., São Paulo, Brazil*

$1967-$

Tomonaga, Y., Tokyo, Japan

Verma, A. R., New Delhi, India*

$1967-1973$

$1967-$

Branscomb, L. M., Washington, D.C., U.S.A.

1969-1972

$1969-$

Honti, P., Budapest, Hungary*

lssaev, B. M., Moscow, U.S.S.R.*

$1969-$

Preston-Thomas, H., Ottawa. Canada*

$1969-$

Djakov, E., Sofia. Bulgaria*

$1970-$

Stille. U., Braumschweic. Gcrmany* (Fed. Rep. of)

$1970-$

Perlstain. A., W abern-Berne, Switzerland*

1971 -

Ambler, E., Washington, D.C., U.S.A.*

$1972-$

Sakurai, Y., Tokyo, Japan *

$1974-$

\section{Chairmen of the CIPM}

lbañez, C.

1875-1891

Foerster, W.

1891-1920

Gautier, R. ${ }^{2}$

1920-1921

Volterra, V.

1921-1940

Zeeman, P. ${ }^{2}$

1940-1943

Sears, J. E. ${ }^{3}$

1946-1954

Danjon, A.

1954-1960

Vieweg, R.

1960-1964

Howlett, L. E.

1964-1968

Otero, J. M.

1968-

\section{Vice-Chairmen of the CIPM}

Crittenden, E. C.

1952-1954

Vieweg, R.

1954-1960

Howlett, L. E.

1960-1964

Otero, J. M.

1964-1968

Dunworth, J. V.

1968-

\footnotetext{
ad interim

${ }^{3}$ ad interin from 1946 to 1948
} 


\section{Secretaries of the CIPM}

Hirsch, A.

1875-1901

Blasema, P.

1901-1918

Hepites, St.-C."

1918-1922

Bodola. L. de

1923-1926

1927-1933

1933-1941

Cabrera. B.

19) $16-195-2$

Dehalu. 11.

$19.52-1962$

Camsinis. G.

$1962-$

\section{Directors of the BIPM}

Govi, G. [Italian]

1875-1877

Pernet, J. [Swiss] ${ }^{5}$

$1877-1879$

Broch, O.-J. [Norwegian]

Benoît, J.-R. [French]

1879-1889

Guillaume, Ch.-Ed. [Swiss]

1889-1915

1915-1936

Pérard, A. [French]

1936-1951

Volet, G. [Sw iss]

1951-1961

Terrien, J. [French]

1962- 



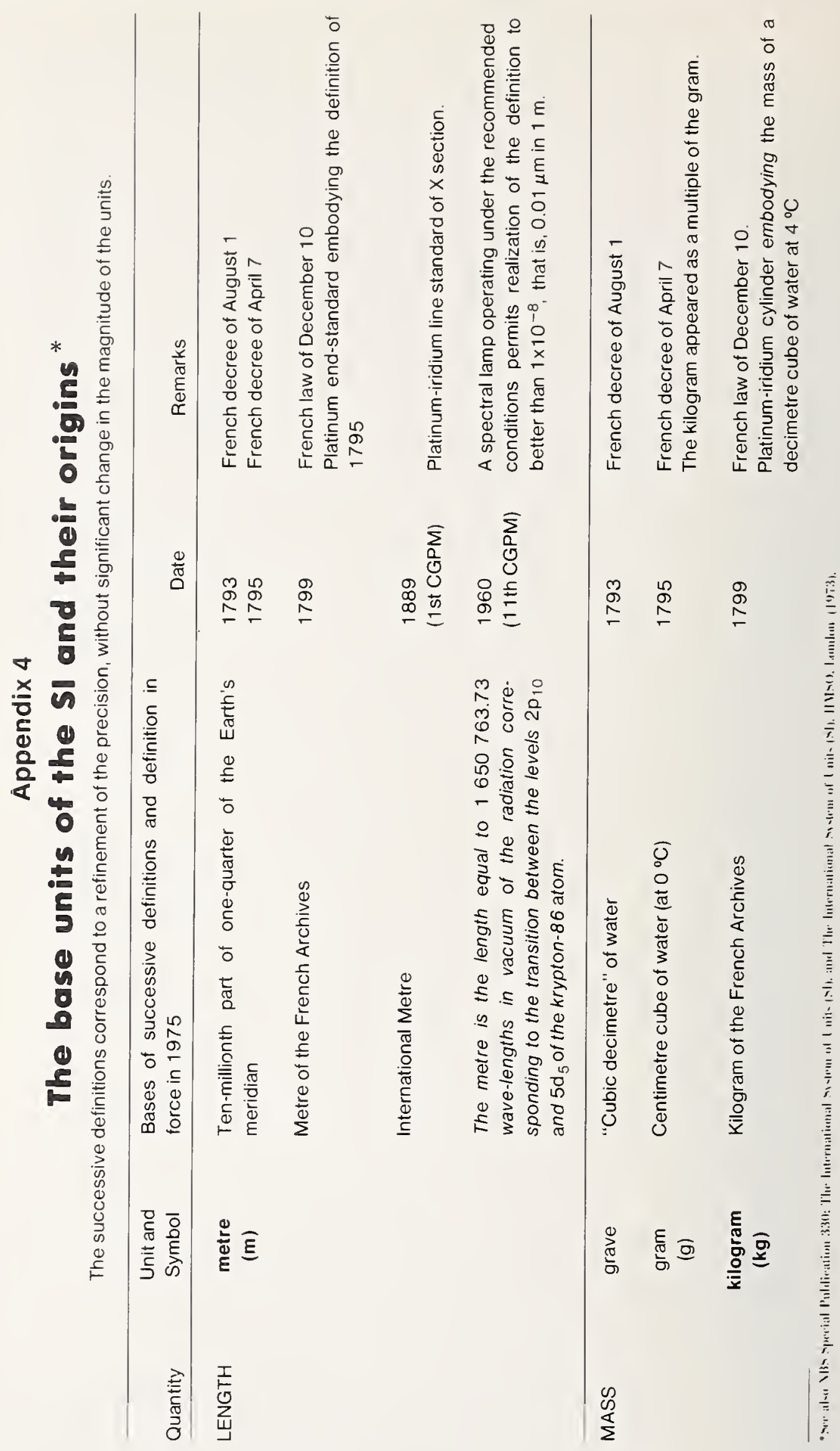




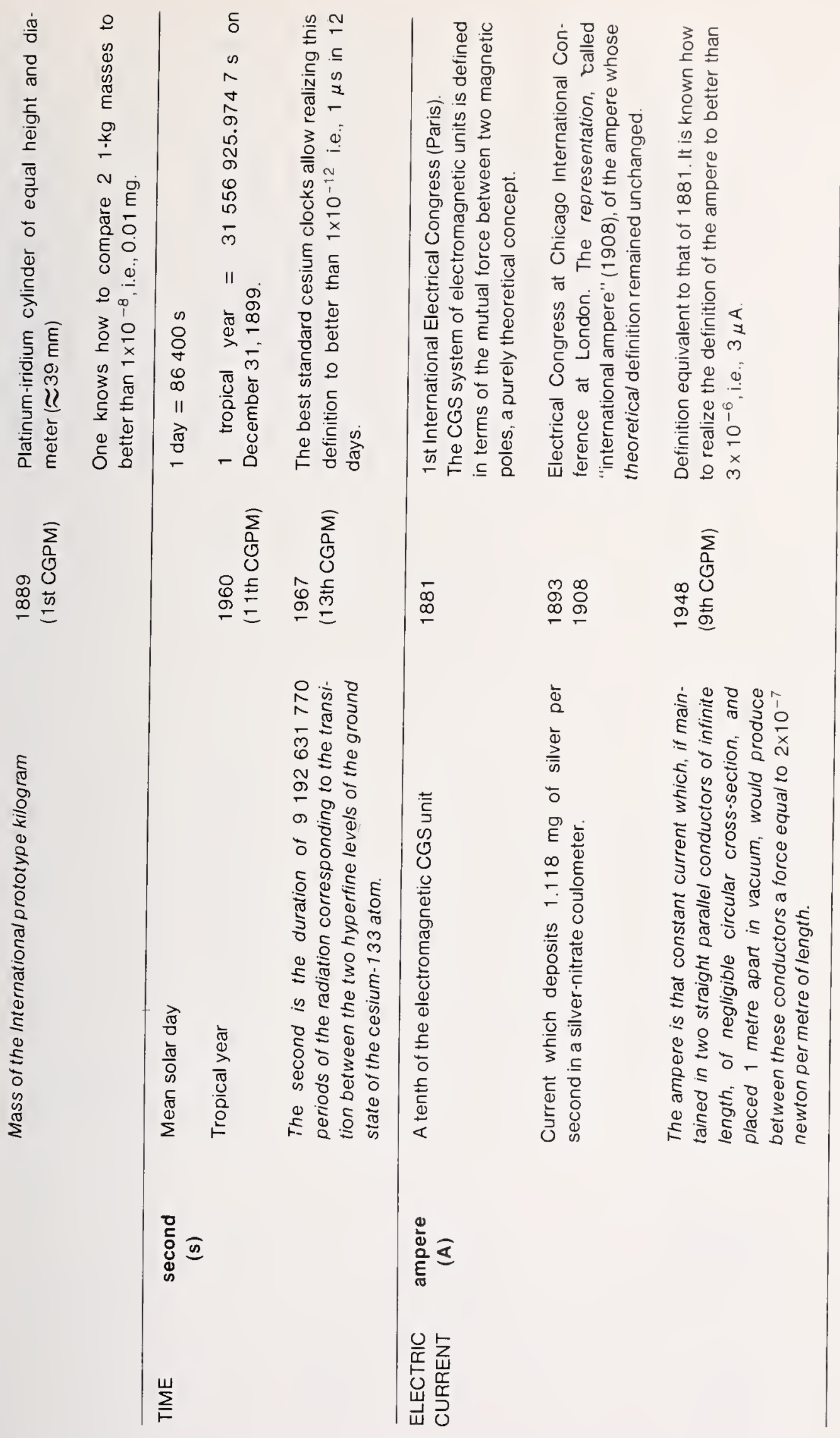




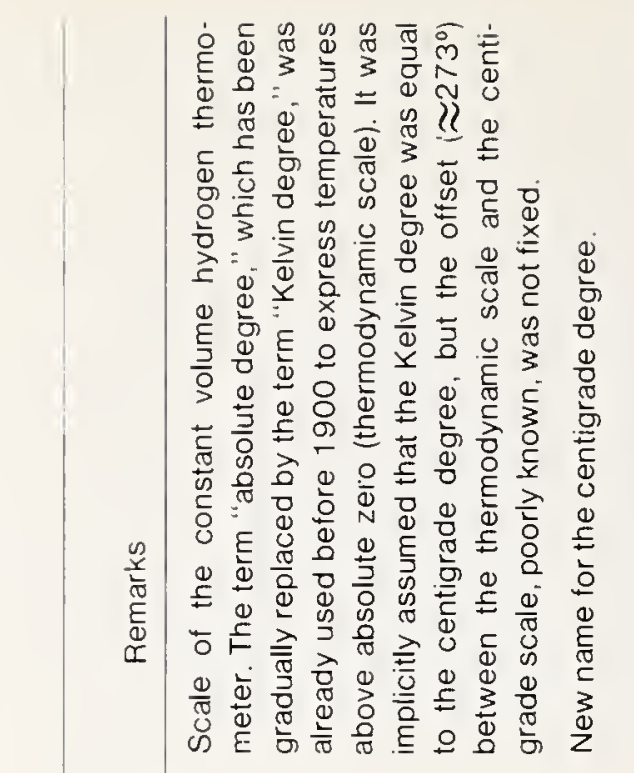

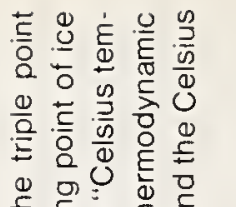

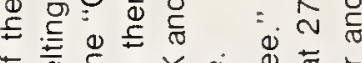

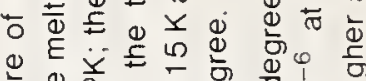

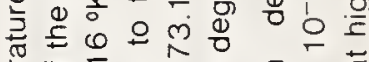

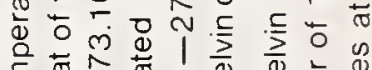

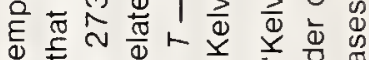

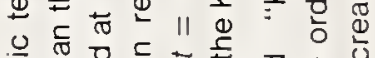

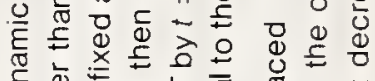

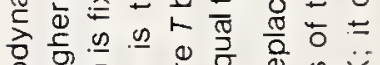

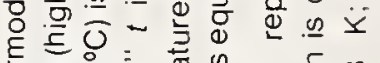
है⿴囗十心

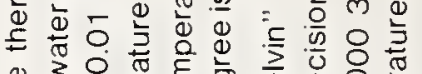

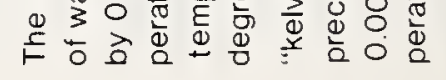
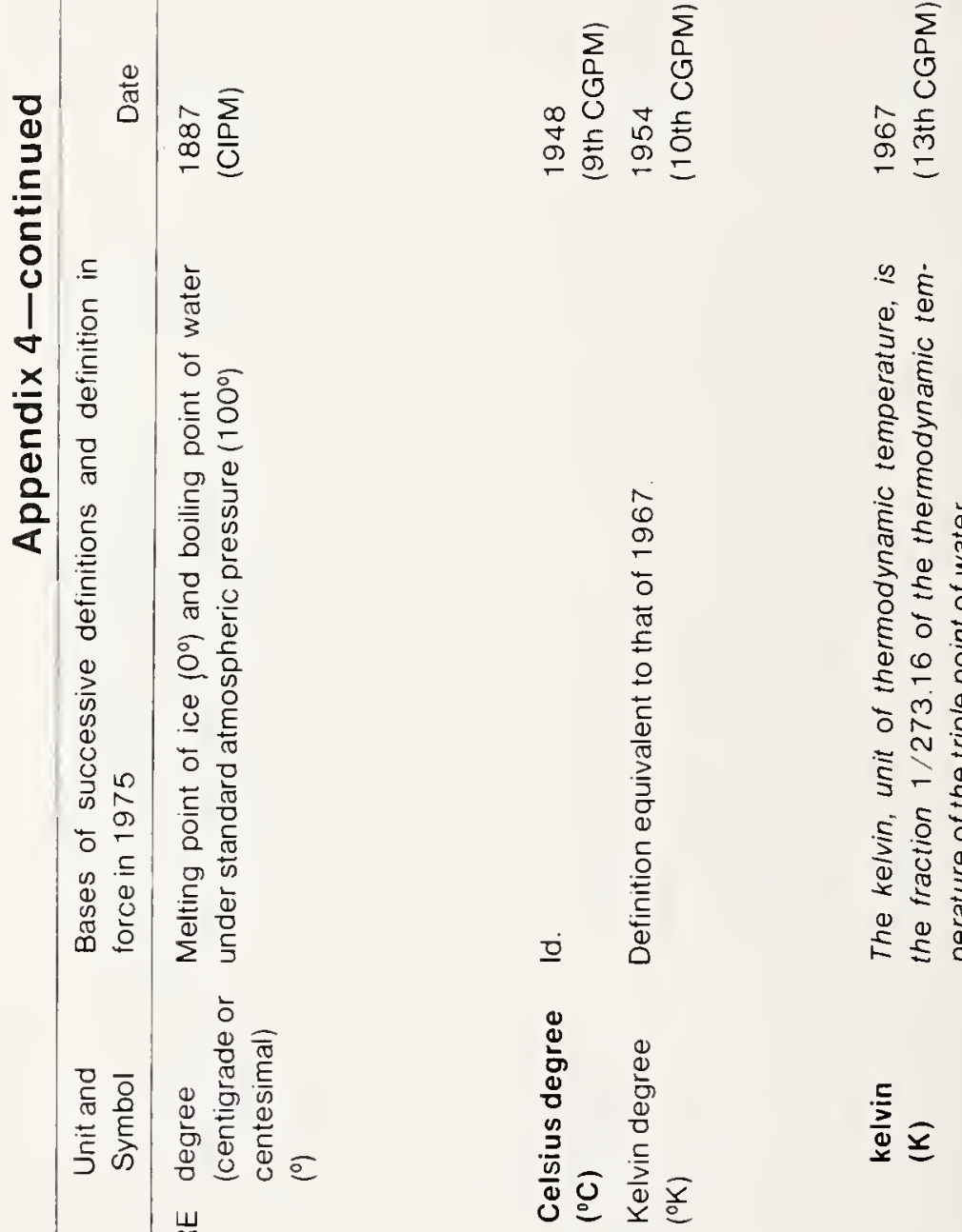

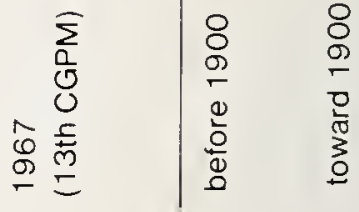

$\frac{6}{6}$

훙

$\stackrel{2}{\circ}$

$\stackrel{5}{2}$

흔

II

음

달ㄹ

Хิ

=

$11 \stackrel{5}{=}$

II

要是

烦高

$\frac{\Phi}{3}$

$\frac{\pi}{\varepsilon} \frac{5}{0}$

$\varepsilon$,

은 $\frac{c}{3} \frac{0}{E}$

3 .

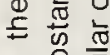

o क

흥

도을

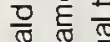

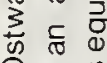
$+\frac{9}{\pi} \stackrel{\frac{0}{\omega}}{\omega}$

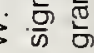
$\frac{\sqrt{2}}{8}$ 


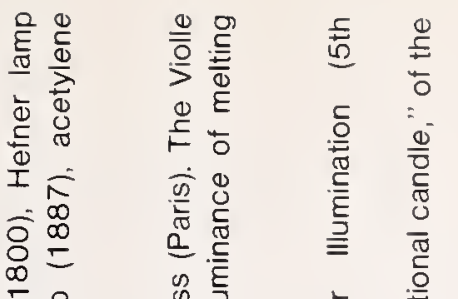

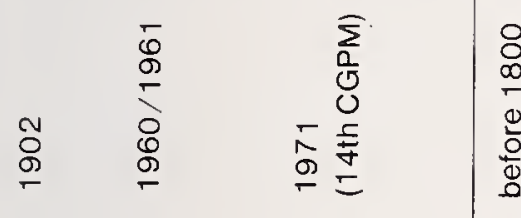

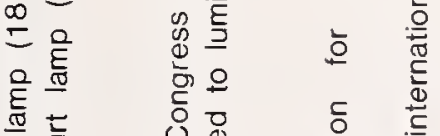

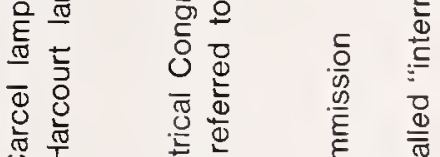

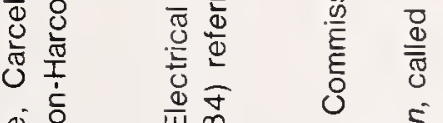

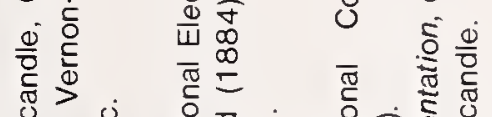

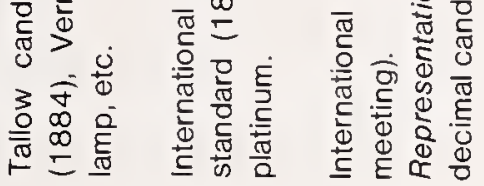

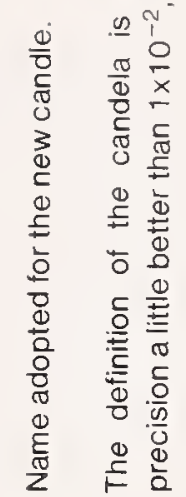

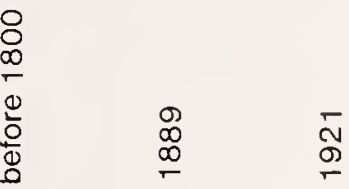

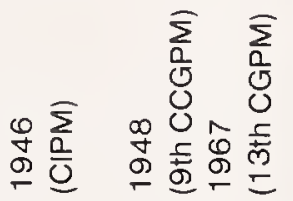

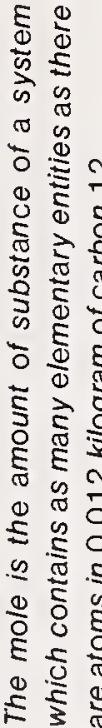

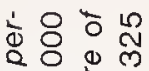

응 웠듬

5 -

등

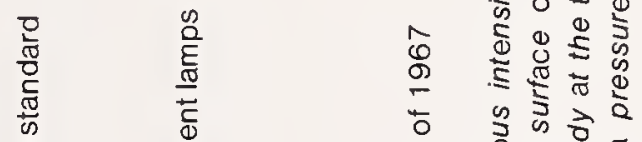

ब.

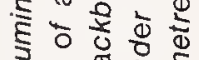

ㄷำ

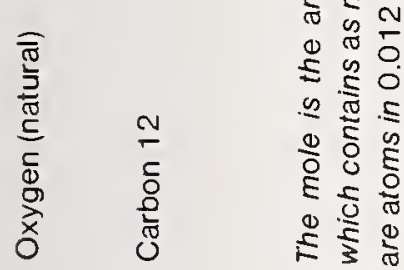

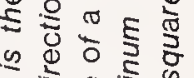

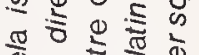

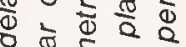

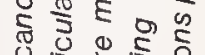

व 동

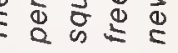

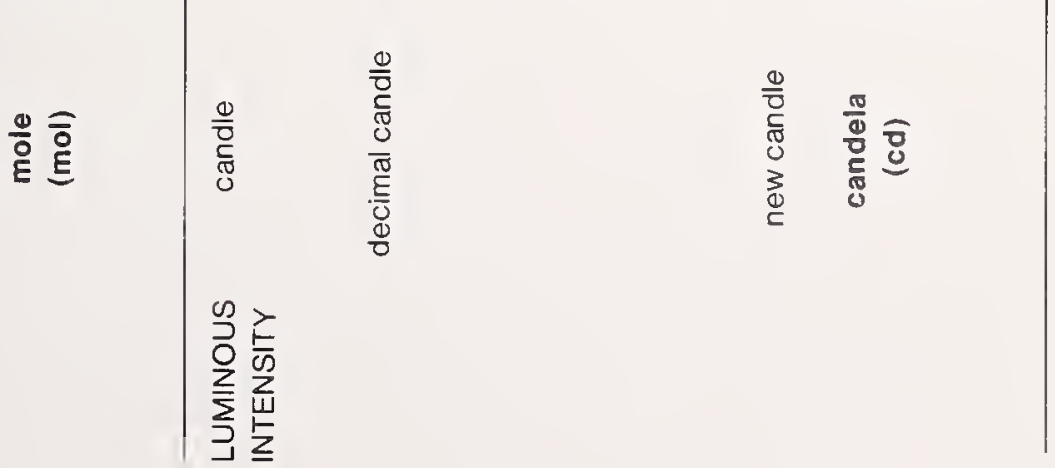





\section{Appendix 5}

\section{Worldwide Expansion of the Metric System}

In 1875, at the time of founding the BIPM, the Metric System had been adopted or introduced into some 30 countries. In 1975, almost all the countries in the world had adopted it or had started a metric reform based on the SI, thus leaving a hope that the world will be 100 percent metric at the end of the 20th century. (See fig. 1 on next page.) 


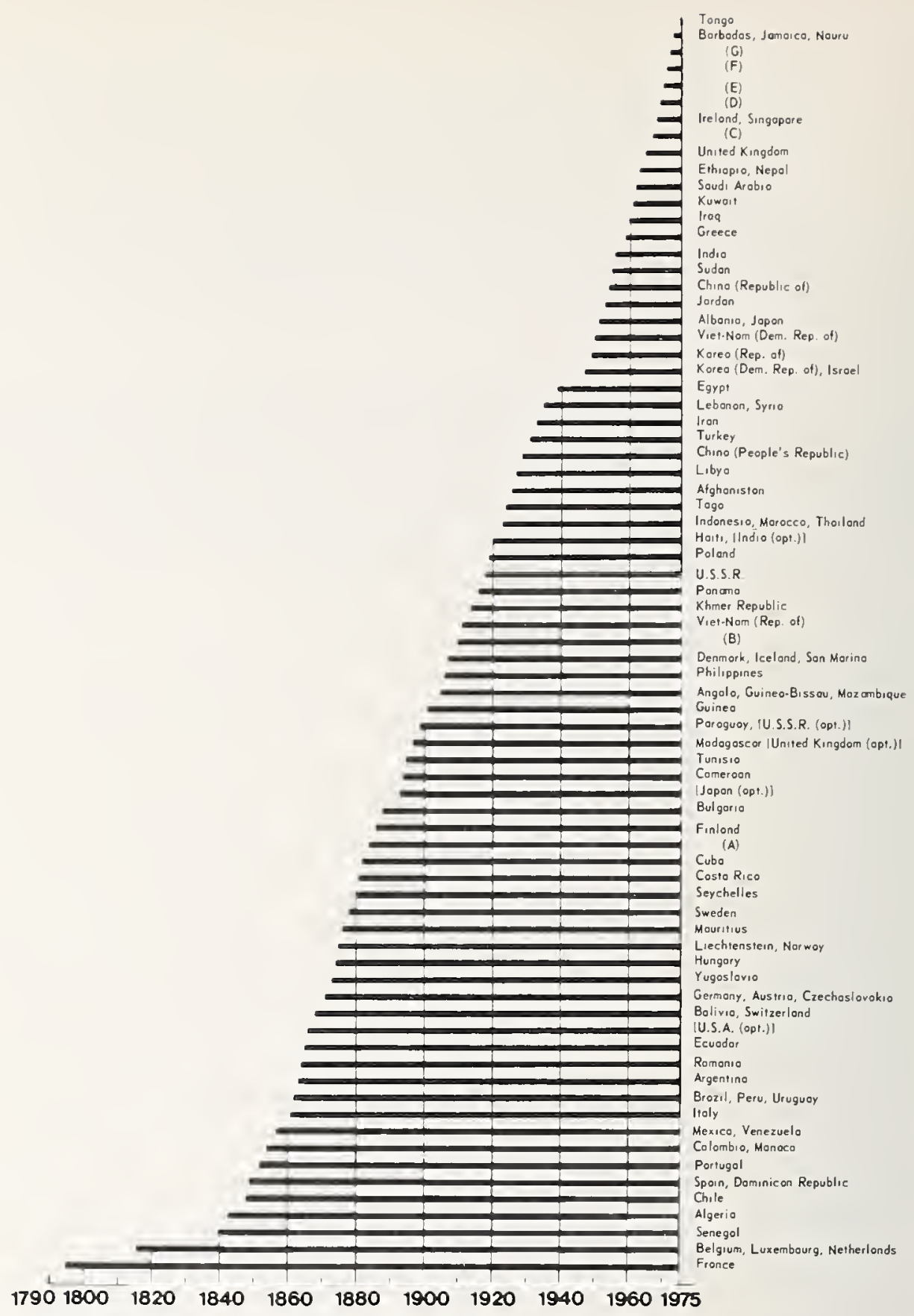

Figure 1. Expansion of the Metric System throughout the world since it creation in 1795. The dates are those of the adoption or introduction of the Metric System in various countries and not those of its complete usage, this often not occurring until after a more or less long delay. A: Central African Republic. Congo (Rep. of the). Ivory Coast, Dahomey, Gabon, Upper Volta, Mali, Mauritania, Niger, Chad; B: Guatemala, Honduras, Malta, Nicaragua. El Salvador, Zaire: C: South Africa, Kenya, Uganda, Pakistan, Tanzania: D: Bahrain, Botswana. New Zealand, Rhodesia. Swaziland: E: Australia. Canada. Gibraltar, Papua and New Guinea, Sri Lanka, Trinidad and Tobago, Zambia; F: Bermuda, Guyana (Rep. of). Malaysia, Nigeria; G: Cyprus, Fiji, Ghana, Somalia. opt: Adoption for optional use. 


\section{Appendix 6}

\section{Publications of the Metre Convention Organs}

The scientific and metrological work accomplished during the last century by the CGPM, the CIPM, its Consultative Committees, and the BIPM is recorded in the following publications which constitute important documentation on scientific metrology.

1. Comptes rendus des séances de la Conférence Générale des Poids et Mesures (14 volumes from 1889 to 1971).

Reports on the work of the CIPM, its Consultative Committees, and the BIPM; general administrative and financial decisions concerning the operation of the BIPM; discussion and adoption of metrological resolutions of international import. (The transactions of the first nine CGPM's are also published in collection 4.)

2. Proces-verbaux des séances du Comité International des Poids et Mesures (65 volumes from 1875 to 1973).

Administration of the BIPM and orientation of its work; detailed report of the director on the activity of the BIPM; examination of reports of the Consultative Committees, results of various international comparisons, and proposals to ve submitted to the CGPM.

3. Consultative Committees (The transactions of each meeting of a Consultative Committee are given in a publication.)

Report of the Consultative Committee (and possibly of its Working Groups) presented to the CIPM; reports on international comparisons carried out at the BIPM or under the auspices of the CIPM; communications on the principal metrological work carried out in the world's specialized laboratories. (Before 1960, these transactions were published as appendices to the Procès-Verbaux du C.I.P.M. The 7 Consultative Committees have held 51 meetings: CCE (13), CCP (7), CCT (10), CCDM (5), CCDS (7), CCU (4), CCEMRI (5 from 1959 to 1964; since 1969, this Consultative Committee has comprised four independent Sections which have held seven meetings: Section I (2), Section II (2), Section III (2), Section IV (1)).

4. Travaux et Mémoires du Bureau International des Poids et Mesures (22 volumes from 1881 to 1966 ).

Detailed descriptions of the principal work and metrological studies 
carried out at the BIPM since its founding. (Publication stopped in 1966 and replaced by the following one.)

5. Recueil de Travaux du Bureau International des Poids et Mesures (4 volumes from 1966 to 1974).

Collection of articles published by the physicists and metrologists of the BIPM in scientific and technical journals or in the form of internal reports.

6. Certain documents published in the preceding collections are also published separately: Récents progrès du Système métrique, Echelle International Pratique de Témperature, Système International d'Unités.

\section{Annual Reports}

Reports from the CIPM office to the Governments on the administrative and financial situation of the BIPM.

All the preceding publications are edited under the care of the BIPM.

The journal Metrologia, founded in 1965 and edited under the auspices of the CIPM, publishes articles on the work of scientific metrology carried out in the world; periodically, information is given on the activities of the Metre Convention organs under the heading "News from the BIPM." 


\section{Appendix 7}

\section{Abbreviations Used in This Volume}

AAEC

AECL

AEE'T

AIEA

ASMW

BCMN

BIPM

CCDM

CCDS

CCE

CCEMRI

CCP

CCPR

$\mathrm{CC} T$

CCU

CENS

CGPM

CIPM

CNAM

DAMW

E'TL

IEN

IKO

Australian Atomic Energy Commission, Lucas Heights, Australia

Atomic Energy of Canada Limited, Chalk River, Canada Atomic Energy Establishment (formerly Bhabha Atomic Research Center), 'Trombay, India

International Atomic Energy Agency, Vienna, Austria

Amt fur Standardisierung, Messwesen und W arenprü-

fung (formerly DAMW), Berlin, German Democratic Republic

Bureau Central de Mesures Nucléaires (Euratom), Geel, Belgium

International Bureau of Weights and Measures, Sèvres, France

Consultative Committee for the Definition of the Metre Consultative Committee for the Definition of the Second Consultative Committee for Electricity

Consultative Committee for the Standards of Measurement of Ionizing Radiations

Consultative Committee for Photometry (now CCPR)

Consultative Committee for Photometry and Radiometry (formerly CCP)

Consultative Committee for Thermometry

Consultative Committee for Units

Centre d'Études Nucléaires de Saclay, Gif-sur-Yvette, France

General Conference for Weights and Measures

International Committee for Weights and Measures

Conservatoire National des Arts et Métiers, Paris, France Deutsches Amt für Messwesen und Warenprüfung (now ASMW), Berlin, German Democratic Republic

Electrotechnical Laboratory, Tokyo, Japan

Istituto Elettrotecnico Nazionale Galileo Ferraris, 'Turin, Italy

Institut voor Kernphysik Onderzoek, Amsterdam, Netherlands 
IMM

IPTS

ISN

ISS

IUPAC

IUPAP

JEN

LCE

LCIE

NBS

NPL

NPRL

NRC.

NRLM

NSL

$\mathrm{OMH}$

PTB

PTR

RIV

SI

UVVVR

Mendeleyev Institute of Metrology, Leningrad, U.S.S.R. International Practical Temperature Scale

Boris Kidrič Nuclear Science Institute, Vinča, Yugoslavia

Istituto Superiore di Sanità, Rome, Italy

International Union of Pure and Applied Chemistry

International Union of Pure and Applied Physics

Junta de Energía Nuclear, Madrid, Spain

Laboratoire Central d'Électricité (now LCIE), Paris, France

Laboratoire Central des Industries Électriques (formerly LCE), Fontenay-aux-Roses, France

National Bureau of Standards, Boulder (B), Gaithersburg (G), U.S.A.

National Physical Laboratory, 'Teddington, England

National Physical Research Laboratory, Pretoria, South Africa

National Research Council, Ottawa, Canada

National Research Laboratory of Metrology, Tokyo, Japan

National Standards Laboratory (now National Measurement Laboratory, NML), Chippendale, Australia

Országos Mérésügyi Hivatal (National Office of Measures), Budapest, Hungary

Physikalisch-Technische Bundesanstalt, Braunschweig, Federal Republic of Germany

Physikalisch-Technische Reichsanstalt (formerly German National Laboratory)

Rijks Institut voor de Volksgenzondheid, Utrecht, Netherlands

International System of Units

Ústav pro Výzkum, Výrobu a Využití Radioisotopù (Institute for Research, Production, and Application of Radioisotopes), Prague, Czechoslovakia

U. S. GOVERNMENT PRINTING OFFICE : 1975 O - 573-106 


